\title{
ABStract \\ Exclusive Muon-Neutrino Charged Current MUON PLUS ANY NUMBER OF PROTONS TOPOLOGIES IN ARGONEUT
}

\author{
KInga Anna Partyka
}

2013

Neutrinos remain among the least understood fundamental particles even after decades of study. As we enter the precision era of neutrino measurements bigger and more sophisticated detectors have emerged. The leading candidate among them is a Liquid Argon Time Projection Chamber (LArTPC) detector technology due to its bubble-like chamber imaging, superb background rejection and scalability. It is a perfect candidate that will aim to answer the remaining questions of the nature of neutrino and perhaps our existence.

Studying neutrinos with a detector that employs detection via beautiful images of neutrino interactions can be both illuminating and surprising. The analysis presented here takes the full advantage of the LArTPC power by exploiting the first topological analysis of charged current muon neutrino $\mu+N p$, muon and any number of protons, interactions with the ArgoNeuT LArTPC experiment on an argon target. The results presented here are the first that address the proton multiplicity at the vertex and the proton kinematics. This study also addresses the importance of nuclear effects in neutrino interactions. Furthermore, the developed here reconstruction techniques present a significant step forward for this technology and can be employed in the future LArTPC detectors. 



\title{
ExClusive MuON-NeUtrino \\ Charged Current muon plus any NUMBER OF PROTONS TOPOLOGIES IN ArgoNeuT
}

\author{
A Dissertation \\ Presented to the Faculty of the Graduate School \\ of \\ Yale University \\ in Candidacy for the Degree of \\ Doctor of Philosophy
}

by

Kinga Anna Partyka

Dissertation Director: Bonnie T. Fleming

December 2013 
(C) 2013 by Kinga Anna Partyka

All rights reserved. 
Dedicated to my parents 


\section{Acknowledgments}

I was once told that the best years of our lives happen in college, clearly, the person who said it did not know about the PhD program. I am so grateful to all the people who made this journey possible. First, I want to thank my amazing parents who made it all possible by sacrificing their careers in Poland and moving to the USA in hope of creating better opportunities for me and my brother. Even the very first page of this thesis would not have been possible without your support - thank you for all you have done for me. I can never repay you but at least I wish to dedicate this thesis to you.

I am grateful to my adviser for convincing me to join her group, it was the best decision I made. Thank you for always supporting me and believing in me. Bonnie, I am so grateful for all the freedom you gave me in doing research, even though this path was more challenging as I was never told what to do next, it made me an independent thinker. Finally, thank you for allowing me to attend so many conferences - they were the highlight of my research and some of the best memories that I have. You set the standard for being a group leader. Thank you for everything.

My journey as part of the ArgoNeuT collaboration would not have been the same without the great support of its members. I am very grateful to Mitch Soderberg for introducing me to the research work within the group. You are one of a few people who can really explain any physics concept in simple words. Mitch, thank you for always finding time for me, always giving me your honest opinion, and being extremely approachable. It felt great knowing that I could always count on you and share any difficulty I encountered. I also want to extend my appreciation to the rest of the ArgoNeuT group, especially to:

- Tingjun Yang for teaching me first steps of performing a neutrino analysis. Thank you for always finding time for me and all your great insight. It was a great pleasure working 
with you.

- Josh Spitz for not only being a good friend but also for teaching me so much about neutrino physics, our "10-minute-sessions" always made me smile - thank you.

- Brian Rebel for teaching me about LArSoft and giving me projects that made me a much better programmer.

- Eric Church for all the help with debugging our software and your patience.

- Carl Bromberg for your constant support and enthusiasm for ArgoNeuT.

- Roxanne Guenette for being a good friend, sharing many funny conversations in our office, and teaching me a few French words.

- Sam Zeller for all your insight and ideas that shaped my research.

- Brian Page for many useful discussions and help with LArSoft.

- Corey Adams for all the encouragement in the final months and listening to me with a smile when I was in a bad mood.

- Andrzej Szelc for your guidance, insight, and honesty. Thank you for all your encouragement in the final months of my work.

- Rashid Mehdiyev for all the help with better understanding of MINOS and many great conversations.

- Flavio Cavanna for the support, many useful physics discussions, and help with my analysis.

Finally, I want to thank Ornella Palamara for being like an "official" adviser to me and all the help with shaping my analysis. We have shared so many great moments together and at times you felt like a mother to me. It was so easy to talk to you about anything from fashion to nuclear effects. Even though we had many sleep-deprived nights, it was all worth it: we developed a successful and original piece of work. Thank you for your engaging conversations, you were invaluable resource to me. Thank you for your limitless energy and enthusiasm in the office and outside. Working with you has been a privilege, you set the standard for being a great researcher. Thank you for everything.

I have so many other inspiring people outside of my collaborations to thank:

- Teppei Katori for your superior knowledge of physics and the joy of sharing it. My summers at Fermilab were always special because I knew you. Thank you for always listening to my stories, letting me sit in your super-comfy chair and your hilarious comments.

- Joe Grange for being a great friend, I could always count on you. Also, thank you for your insightful neutrino discussions. 
- Jorge Morfin for being one of the nicest physicists I have ever met. Even though we were never part of the same collaboration you always made me feel welcomed. Thank you for many useful discussions. Also, thank your for making me feel like I was working in a high-fashion office whenever I saw you on the 10th floor.

- MINOS Collaboration for providing the reconstruction of muons exiting ArgoNeuT.

- GENIE authors, in particular S. Dytman and H. Gallagher, for many useful discussions. My list here is in no way complete, but I would probably have to spent more time listing everyone than it took to write my entire thesis so I choose not to go on. I briefly want to thank my whole incoming class of 2007 for your support, our first year was awful with countless hours of solving problems but we made it! Finally, I would like to thank my close friends outside of the physics world: you helped me reach my goal through your constant support. Thank you! 


\section{Contents}

Acknowledgments $\quad$ i

Contents iv

List of Figures viii

List of Tables $\quad$ xxii

1 Introduction to the Dissertation $\quad 1$

I DESCRIPTION OF THE NEUTRINO 3

2 Neutrino Overview $\quad 4$

2.1 The Birth of the Neutrino . . . . . . . . . . . . 4

2.2 Detection of the Neutrino $\ldots \ldots \ldots \ldots \ldots$

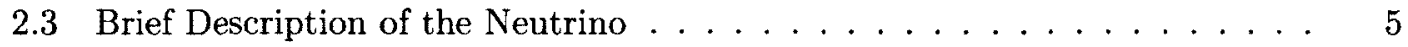

2.3.1 Parity Violation and Handedness . . . . . . . . . . . 6

2.4 Neutrino Sources . . . . . . . . . . . . . . . . . . . 7

3 Neutrino Oscillation $\quad 10$

3.1 Neutrino Oscillation in Vacuum . . . . . . . . . . . . . . . . 10

3.1.1 Features of Neutrino Oscillation . . . . . . . . . . . . 13

3.1 .2 Two neutrino mixing . . . . . . . . . . . . . . 14

3.1 .3 Three neutrino mixing . . . . . . . . . . . . . . 14

3.2 Neutrino Oscillation in Matter f . . . . . . . . . . . 15 
3.3 Adding Mass Term to the Standard Model . . . . . . . . . . . . . . . . 17

3.4 The Evidence for Neutrino Oscillation . . . . . . . . . . . . . . . . 18

3.4 .1 Solar neutrino oscillations . . . . . . . . . . . . . 18

3.4 .2 Reactor neutrino oscillations . . . . . . . . . . . . 20

3.4 .3 Atmospheric neutrino oscillations . . . . . . . . . . . . . 21

3.4 .4 Accelerator neutrino oscillations . . . . . . . . . . . . . 23

3.5 Summary and Outstanding Questions . . . . . . . . . . . . 26

$4 \quad$ Neutrino Scattering $\quad 31$

4.1 Cross Section . . . . . . . . . . . . . . . . . . . 31

4.2 Charged Current Quasi-Elastic Scattering . . . . . . . . . . . . . 34

4.3 Nuclear Effects . . . . . . . . . . . . . . . . . . 37

4.3.1 Final State Interactions . . . . . . . . . . . . . . . 37

4.3.2 Meson Exchange Currents . . . . . . . . . . . . . . . . 38

II Neutrino Detection with the Liquid Argon Time Projection Chamber Technique 40

5 The LArTPC concept $\quad 4 \mathbf{4 1}$

$6 \quad$ Event Imagining and Display $\quad \mathbf{4 5}$

7 Future of the LArTPCs in the US $\quad 50$

7.1 MicroBooNE . . . . . . . . . . . . . . . . . 50

7.2 Next Generation Long Baseline Oscillation Experiment . . . . . . . . . . . 52

III The Argoneut Experiment 53

8 The NuMI Neutrino Beam $\quad \mathbf{5 4}$

9 ArgoNeuT Hardware $\quad \mathbf{5 8}$

9.1 Cryostat and Cryocooler . . . . . . . . . . . . . . . . . 58

9.2 Time Projection Chamber . . . . . . . . . . . . . . . . . 61

9.3 Electronics Readout $\ldots \ldots \ldots \ldots \ldots$. . . . . . . . . . . 63

9.4 Liquid Argon Purification . . . . . . . . . . . . . . . . . . . 66 
9.5 Measuring Liquid Argon Purity . . . . . . . . . . . . . . . . . 68

9.6 MINOS Near Detector . . . . . . . . . . . . . . . . . . 71

10 Commissioning and Data Taking $\quad 74$

11 Detector Performance $\quad 77$

11.1 Electron drift velocity $\ldots \ldots \ldots \ldots \ldots \ldots \ldots$

11.2 Electron lifetime $\ldots \ldots \ldots \ldots \ldots \ldots$

\section{Argoneut Simulation and Reconstruction of Events} 83

12 Simulation $\quad \mathbf{8 4}$

12.1 GENIE Event Generator . . . . . . . . . . . . . . . . . . . 84

12.1.1 GENIE Cross Section Model _ . . . . . . . . . . . . 85

12.1.2 Nuclear Physics Model in GENIE . . . . . . . . . . . . . . 87

12.1.3 GENIE Output . . . . . . . . . . . . . . . . 91

12.2 ArgoneuT Detector Geometry and Signal Simulation . . . . . . . . . . . 91

12.3 Propagation into MINOS Near Detector . . . . . . . . . . . . . . 93

13 LArSoft Software $\quad \mathbf{9 5}$

13.1 Raw Waveform Treatment $\ldots \ldots \ldots \ldots$

13.2 Hit Identification $\ldots \ldots \ldots \ldots \ldots \ldots$

13.32 2-dimensional Clustering . . . . . . . . . . . . . . 98

13.4 2-dimensional Line Reconstruction . . . . . . . . . . . . . 100

13.5 Vertex Finding . . . . . . . . . . . . . . . . . . . 103

13.6 2-dimensional, More Refined Clustering of Short Vertex Tracks . . . . . . 104

13.7 3-dimensional Track Reconstruction . . . . . . . . . . . . . . . 109

13.8 Calorimetric Reconstruction . . . . . . . . . . . . . . . . . . . . . 109

13.9 Matching with MINOS Near Detector . . . . . . . . . . . . . . 111

V ANAlysis $\quad 114$

14 Analysis of $\mu+N p$ Neutrino Interactions $\quad 115$ 
14.1 Introduction and Importance of $\mu+N p$ Topological Measurement $\ldots \ldots .115$

14.2 Analysis Steps . . . . . . . . . . . . . . . . . 117

14.2.1 Setting the Automated Cuts . . . . . . . . . . . . . . 119

14.2.2 Proton Reconstruction Threshold . . . . . . . . . . . . 136

14.2 .3 Visual Scanning . . . . . . . . . . . . . . . 137

14.2.4 Two Ways of Approach . . . . . . . . . . . . . . . . 143

14.2.5 Calorimetric Reconstruction and Particle Identification . . . . . . 144

14.3 Efficiencies . . . . . . . . . . . . . . . . . . . 153

14.4 Background . . . . . . . . . . . . . . . . 156

14.5 Flux . . . . . . . . . . . . . . . . . . . . . 159

14.6 Protons on Target . . . . . . . . . . . . . . . . . . . 159

14.7 Results . . . . . . . . . . . . . . . . . . . 160

14.7.1 Proton multiplicity . . . . . . . . . . . . . . 160

14.7.2 Muon Kinematics . . . . . . . . . . . . . . . . 166

14.7.3 Proton Kinematics . . . . . . . . . . . . . . . . 172

14.8 Concluding Remarks . . . . . . . . . . . . . . . . 177

$\begin{array}{ll}\text { Bibliography } & 179\end{array}$ 


\section{List of Figures}

2.1 Neutrino sources as a function of energy. The electroweak cross-section for $\bar{\nu}_{e} e^{-} \rightarrow \bar{\nu}_{e} e^{-}$is shown for comparison. (from Reference [60]) . . . 8

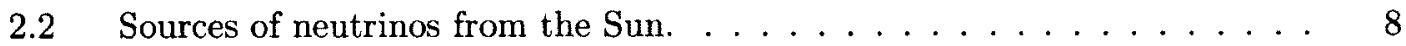

2.3 Sources of neutrinos in atmospheric and accelerator experiments. . . . . 8

3.1 The energy spectra for various processes in the Sun. The energy range covered by different experimental techniques is also displayed. . . . . . . 19

3.2 KamLAND's ratio of observed to expected anti-neutrino rates as a function of average L/E. Since neutrino sources are at different baselines, KamLAND assigns all the events to an average $L . \ldots \ldots \ldots 21$

3.3 Allowed region for the solar oscillation parameters. . . . . . . . . . . . 22

3.4 The zenith angle distribution for e-like and $\mu$-like events in sub-GeV and multi-GeV visible energy in Super-Kamiokande. The dotted histograms show the non-oscillated Monte Carlo events. The solid histograms show the best-fit expectations for $\nu_{\mu} \rightarrow \nu_{\tau}$ oscillations. . . . . . . .

3.5 The ratio of the data to Monte Carlo prediction without oscillations as a function of L/E in Super-Kamiokande. The solid histograms show the best-fit expectations for $\nu_{\mu} \rightarrow \nu_{\tau}$ oscillations. . . . . . . . . .

3.6 The ratio of energy spectrum in the far detector to the one in the near detector (after oscillation/before oscillation) . . . . . . . . . . 
3.7 The energy spectrum of MINOS charged current events in the far detector. The red line represents the spectrum in the absence of oscillations. The blue line represents a fit to a 2 -flavour oscillation model. The plot of on the right shows the fraction of data to expected data without oscillations. The plot has a characteristic oscillation behaviour. It is seen that the neutrino oscillation hypothesis best fits the data. . . . . . . . . . .

3.8 A three-neutrino squared-mass spectrum accounting for the observed solar, reactor, atmospheric and accelerator neutrinos. The spectrum on the left is referred to as "normal" and on the right as "inverted" hierarchy. Oscillation experiments cannot determine how far above zero the entire spectrum lies.

4.1 Lowest order Feynman diagram for inverse muon decay . . . . . . . . . .

4.2 Total cross section per nucleon divided by neutrino energy and plotted as a function of energy for neutrinos (top) and anti-neutrinos (bottom). Contributions from Quasi-Elastic, Resonant and Deep Inelastic Scattering processes are shown as modeled by NUANCE generator [40]. The figure is adopted from $[60] \ldots \ldots \ldots \ldots \ldots \ldots$

4.3 Quasi-Elastic scattering cross section as a function of neutrino energy for neutrinos. A variety of different measurements together with free nucleon scattering prediction with $M_{A}=1.0 \mathrm{GeV}$ are shown. The figure is adopted directly from $[60] \ldots \ldots \ldots \ldots \ldots \ldots$

4.4 Quasi-Elastic scattering cross section as a function of neutrino energy for anti-neutrinos. A variety of different measurements together with free nucleon scattering prediction with $M_{A}=1.0 \mathrm{GeV}$ are shown. The figure is adopted directly from $[60] \ldots \ldots \ldots \ldots \ldots$ 
5.1 The LArTPC concept for neutrino detection showing the TPC filled with liquid argon. A neutrino comes in and interacts with an argon atom. The charged particles produced in the interaction ionize the argon atom. The ionization charge is drifted to the wire planes by the electric field. It induces wire signals on "induction" and "collection" plane, where it is finally collected. Each plane produces a two dimensional view of the event in terms of wire number and time of detection. Combining information from all instrumented planes allows for three dimensional reconstruction of the

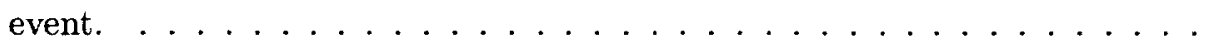

5.2 Chosen properties of the stable noble elements. . . . . . . . . .

6.1 A schematic of the ArgoNeuT reference frames used for 2D and 3D imaging of the ionization events. The induction plane coordinates are $(v, t)$ and $(w, t)$ for the collection plane and are indicated in terms of wire index $n_{W}^{I, C}$, wire pitch $\delta s$, time tick index $n_{t}$, and sampling time $\delta t . \ldots \ldots$

6.2 A CC $\nu_{\mu}$ candidate event with two tracks clearly visible. The longest track corresponds to a MIP-like particle escaping ArgoNeuT TPC. The short track is a more densely ionizing particle as seen by the red trail. . . . . . .

6.3 A CC $\nu_{\mu}$ candidate event with four tracks. The longest two track correspond to a MIP-like particle escaping ArgoNeuT TPC. The two short tracks are highly ionizing (proton-like) and associated with the interaction vertex. . . . . . . . . . . . . . . . .

6.4 A neutrino interaction featuring four well separated showers visible in both planes, and three other tracks originating at the interaction vertex. . . . .

7.1 Comparison of ArgoNeuT and MicroBooNE detector specifications. . . . .

7.2 Installations of MicroBooNE TPC. The size of the detector with respect to people can be easily referred. . . . . . . . . . . . . . . . .

8.1 Two-lens focusing system for the NuMI line. The second horn improves the collection efficiency of partciles coming from the first horn that were overor underfocused by the first horn. . . . . . . . . . . . . .

8.2 Plain and elevation view of the NuMI beam. ArgoNeuT detector was located right in front of MINOS Near Detector in the "near detector hall". . 
8.3 Neutrino beam energy spectra with the target in its nominal position inside the horn, "low energy" or retracted $1 \mathrm{~m}$ ("medium energy") or $2.5 \mathrm{~m}$ ("high energy" $\ldots \ldots \ldots \ldots \ldots \ldots \ldots \ldots \ldots$

9.1 [Left] ArgoNeuT detector in the neutrino beam line placed in front of MINOS Near Detector. The cryostat is placed in the orange box, whose purpose is to contain the liquid in case of a major spill. [Right] Aerial view of the Fermilab with MINOS Near Detector Hall pointed out. . . . . . . . .

9.2 [Left] ArgoNeuT cryostat cross-section views. The drawings show the inner and outer vessels, end-caps, and chimney on the top. [Right] Picture of the cryostat taken during the installation. . . . . . . . . . . .

9.3 A screenshot of the remotely controllable cryo-system monitoring software. The display shows the relevant parameters of the system during operation.

9.4 Relative orientation of wires on shield, induction and collection planes. Shield plane is not instrumented for the readout. . . . . . . . . . . . 62

9.5 The ArgoNeuT TPC about to enter the inner vessel of the cryostat through the removed front-end cap. . . . . . . . . . . . . . . .

9.6 [Left] The ADF-2 digitizer card sampling 2048 samples/channel. [Middle] The preamplifier and filter card (PFC). [Right] A set of bias voltage cards (BVDC) that are positioned on the TPC frame. . . . . . . . . . . 64

9.7 A few relevant electronics readout specifications. . . . . . . . . . . . 64

9.8 The readout signal feedthrough flange inside the RF shielded cage positioned on the outside of the cryostat. The box with PFC in their crate and pleated foil cables to the digitizer board are shown on the right. . . . . . 65

9.9 The ArgoNeuT recirculation system featuring filters and the cryocooler. . 68

9.10 The ArgoNeuT filter cartridges serving for liquid argon purification. . . . 69

9.11 The concept of the purity monitor. A measure of purity of liquid argon is determined from comparing the signals of the anode and cathode after electron from photo electric effect drift the full distance between them. . . 69

9.12 The ArgoNeuT purity monitor positioned on top of the TPC . . . . . 70 
9.13 A diagram of one plane of MINOS Near Detector $3.8 \mathrm{~m}$ tall and $4.8 \mathrm{~m}$ wide. An electromagnetic coil passes through the hole in the center of each plane. Some planes are fully instrumented while others only on the side hit by the beam as shown by the diamond-shaped region. . . . . . . . . . 72

9.14 The MINOS scintillator strip composition. . . . . . . . . . . . 73

10.1 The ArgoNeuT physics run in terms of delivered and acquired protons on target (POT) as a function of time. The two week stop in operation was due to a failure of a commercial component of the cooling system. . . . . 76

11.1 The left plot shows hit time distribution from the Induction plane for neutrino induced through-going muon tracks. The sample includes tracks crossing either the cathode or the Induction plane of the TPC. These tracks provide the hits at the edges of the distribution and thus determine the drift time measurement. The plot on the right shows the drift velocity measurement in ArgoNeuT, and associated systematic uncertainties, and comparison with expectation values from [115] as a function of the electric field and for different LAr temperatures corresponding to the range of variation of the current LAr temperature during operations. . . . . .

11.2 A 2-dimensional scatter plot of signal height as a function of drift time showing every hit associated with a long track on the collection plane in neutrino mode running. . . . . . . . . . . . . . .

11.3 The e-lifetime extraction from the Collection plane with an exponential fit to the data. The fit gives an e-lifetime of $764 \pm 3_{\text {stat }} \mu$ s (statistical error only) for a specific DAQ-run in neutrino mode. . . . . . . . . . . .

11.4 The e-lifetime as a function of run number for the entire ArgoNeuT data taking period. $\ldots \ldots \ldots \ldots \ldots \ldots \ldots \ldots \ldots \ldots \ldots \ldots \ldots \ldots$

11.5 The $\mathrm{O}_{2}$ equivalent impurity concentration in LAr (ppb) extracted from each DAQ-run as a function of elapsed time (days) since the start of the ArgoNeuT physics run (about 160 days, beam in neutrino and antineutrinomode). Vertical lines and band indicate hardware interventions on the purification/recirculation system: [red dotted] Filter exchange, [green dotted] GAr purge, [gray shaded] replacement of a cryocooler component - recirculation was halted during this period (about two weeks) and DAQ paused. 
12.1 A Charged Current Quasi-Elastic (CCQE) interaction . . . . . . . 85

12.2 A neutral current elastic interaction . . . . . . . . . . 86

12.3 A charged current coherent (left) and neutral (right) interaction . . . . 87

12.4 A charged current DIS (left) and neutral DIS (right) interaction . . . . 87

12.5 A charged current single pion resonat (left) and neutral single pion resonant (right) interaction ..................... 88

12.6 Schematic diagram for reaction involving FSI process (left) and reaction of the pion absorption (right) . . . . . . . . . . . . . . . . 90

12.7 The ArgoNeuT TPC in Geant4 with the wire planes in the foreground (top). A few wires (in white) are shown to see their relative angles to one another. The cross section of the whole ArgoNeuT detector is seen in the bottom plot. Starting from the middle and going outwards, the TPC, the inner and outer cryostats are seen. The detector sits in an orange containment vessel. . . . . . . . . . . . . . . . . . . . . .

12.8 The simulation of the ArgoNeuT detector together with the first MINOS plane. . . . . . . . . . . . . . . . . . .

13.1 Current signals for the Induction and Collection wires as induced by a drifting charge. . . . . . . . . . . . . . . . 96

13.2 Wire raw waveform shown in black and deconvoluted waveform shown in blue for a crossing muon track in data, parallel to the wire planes. . . . .

13.3 Induction plane view of two tracks (upper left) with wire views on three adjacent wires. The raw data is shown in black and the deconvoluted data in red. . . . . . . . . . . . . . . . . . . . . 99

13.4 Core points and border points (adopted from Reference [112]) . . . . . . 100

13.5 Density-reachability and density-connectivity (adopted from Reference [112]) 100

13.6 The DBSCAN clustering algorithm ran on a neutrino event. The display shows colored hits on the induction and collection plane, each color corresponds to a unique cluster formed. . . . . . . . . . . . . . . . 101 
13.7 The DBSCAN clustering algorithm ran on a neutrino event. The display shows colored hits on the collection plane, each color corresponds to a unique cluster formed. The red hits represent noise as decided by DBSCAN and are not passed to other algorithms. . . . . . . . . . . . 101

13.8 Representation of two points in $(\mathrm{x}, \mathrm{y})$ plane and their parametrization in $(\mathrm{r}, \theta)$ space. The crossing curve in $(\mathrm{r}, \theta)$ corresponds to the line passing through both points in $(\mathrm{x}, \mathrm{y}) . \ldots \ldots \ldots \ldots$

13.9 2-dimensional clustering with Hough Transform on a ArgoNeuT neutrino event. The reconstructed hits are visible in gray, while the two colors represent the clustered lines corresponding to 2-dimensional tracks. . . . .

13.10 A 2-track data event shown in collection plane. Origin of the coordinate system is set on a reconstructed vertex, each hit reconstructed by DBSCAN has its angle theta calculated as indicated. . . . . . . . . . . .

13.11 A plot of number of hits as a function of theta angle performed for the data event shown on the right. Clusters are formed from the two peaks clearly visible in the plot. The hits not falling into either cluster are assigned based on their proximity. The colored hits correspond to individual clusters formed (green and red). . . . . . . . . . . . . . .

13.12 The event display showing LineMerger (top) and VCluster (bottom) ran on the same data event. In this case LineMerger merges two short tracks as one on top plane and fails to recognize a 2-hit cluster on the bottom plane while VCluster reconstructs both. . . . . . . . . . . . . 106

13.13 The event display showing LineMerger (top) and VCluster (bottom) ran on the same data event. In this case LineMerger merges two tracks as one on top plane while VCluster reconstructs them as separate clusters. . . . .

13.14 The event display showing LineMerger (top) and VCluster (bottom) ran on the same data event. In this case LineMerger does not reconstruct the short track coming from the vertex in the top plane while VCluster forms a cluster. . . . . . . . . . . . . . . . .

13.15 Different views of a reconstructed neutrino event in three dimensions. The longest track is seen to be exiting the TPC volume through the cathode plane. 
13.16 Simulated energy loss per unit track length $(\mathrm{dE} / \mathrm{dx})$ as a function of residual range (distance to the track end) for different stopping particles. The black points correspond to the experimental data for the hits along the track shown on the right. They nicely agree with the proton hypothesis. . . . . 111

13.17 The front view of the ArgoNeuT and MINOS detectors from the GEANT4 Simulation (top). The ArgoNeuT TPC is shown in pink. The beam center, origin of the MINOS and ArgoNeuT coordinate system is shown. A matching muon scenario is shown where one track in ArgoNeuT has multiple candidate tracks from MINOS (bottom) $\ldots \ldots \ldots \ldots \ldots$

14.1 The Monte Carlo simulation of the energy spectrum for neutrinos and antineutrinos in anti-neutrino mode in the NuMI beam line. . . . . . . . 118

14.2 The Monte Carlo simulation of the energy spectrum for neutrinos and $\mu^{-}+$ $N p$ events. . . . . . . . . . . . . . . . 118

14.3 The Monte Carlo simulation of the energy spectrum for antineutrinos and

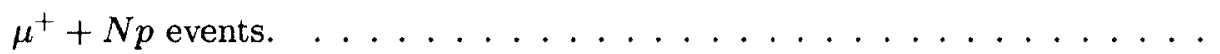

14.4 Comparison of true and reconstructed value of the $\mathrm{X}, \mathrm{Y}$, and $\mathrm{Z}$ vertex coordinates for $\mu^{+}+\mathrm{Np}$ events that pass all automated cuts. . . . . . . .

14.5 Comparison of true and reconstructed value of the $\mathrm{X}, \mathrm{Y}$, and $\mathrm{Z}$ vertex coordinates for $\mu^{-}+\mathrm{Np}$ events that pass all automated cuts. . . . . . 122

14.6 The distribution of vertices for all data events that pass automated cuts. Neutrinos on the left and anti-neutrinos on the right. There are throughgoing muons entering the upstream end of the TPC which are eliminated later in the process of visual scanning. . . . . . . . . . . . . 123

14.7 The distribution of vertices for all data events that pass automated cuts in the $\mathrm{X} / \mathrm{Y}$ (left) and $\mathrm{X} / \mathrm{Z}$ (right) two dimensional views. . . . . . . . . .

14.8 The area normalized distribution of vertices for all data events after scanning and comparison with the MC true $\mu+\mathrm{Np}$ events after automated cuts. The left column shows $\mathrm{X}, \mathrm{Y}$, and $\mathrm{Z}$ distributions for neutrinos and right column for anti-neutrinos. . . . . . . . . . . . . . . . 
14.9 Schematic of three regions through which a muon travels. Region 1 is the TPC, region 2 is between the edge of the TPC and MINOS first plane, and region 3 is inside MINOS Near Detector. The energy loss in liquid argon, in region 1 , is determined from the calorimetry. The energy loss in region 2 is determined from the Geant 4 simulation and in region 3 directly from MINOS. . . . . . . . . . . . . . . . . . . . . .

14.10 The distance between the muon TPC exit point and the point where it reaches the MINOS first plane as a function of energy lost. The shape is attributed to the complicated composition of region 2, consisting of nonuniform thickness and shape of stainless steel parts as a function of distance. 126

14.11 Schematic of ArgoNeuT detector (in orange box) in front of MINOS Near Detector. The image shows the relative size and positioning of the two detectors. . . . . . . . . . . . . . . . . 127

14.12 Display of the ArgoNeuT and MINOS Near Detector (MND) geometries together with a 3-dimensional tracks reconstructed in the TPC. A throughgoing track in ArgoNeuT has been matched to a negatively charged track in the MND. Other tracks present in the MND during the spill are also represented.

14.13 Difference between the projected ArgoNeuT track and the MINOS track in terms of angle (top) and radial position (bottom) for $\mu+\mathrm{Np}$ events ( $\mu^{-}$ on left and $\mu^{+}$on right) passing all the automated cuts. In case of data this refers to events after scanning, and true $\mu+\mathrm{Np}$ after automated cuts in case of MC. Plots are area normalized, errors are statistical only and no background subtraction has been applied to the data. . . . . . . . . 128

14.14 The distance in X(top) and $\mathrm{Y}$ (bottom) between matched MINOS track and the projected ArgoNeuT track for $\mu+\mathrm{Np}$ events ( $\mu^{-}$on left and $\mu^{+}$on right) passing all the automated cuts. In case of data this refers to events after scanning, and true $\mu+\mathrm{Np}$ after automated cuts in case of MC. Plots are area normalized, errors are statistical only and no background subtraction has been applied to the data. . . . . . . . . . . . 
14.15 The angles between matched MINOS track and the projected ArgoNeuT track for $\mu+\mathrm{Np}$ events ( $\mu^{-}$on left and $\mu^{+}$on right) passing all the automated cuts. In case of data this refers to events after scanning, and true $\mu+\mathrm{Np}$ after automated cuts in case of MC. Plots are area normalized, errors are statistical only and no background subtraction has been applied to the data . . . . . . . . . . . . . . . . . . . . . . .

14.16 A data event showing requirement of 'vertex cluster' being a rectangle centered on a vertex and having dimensions of 90 ticks by 6 wires. In this case, three clusters are counted as 'vertex clusters'.

14.17 Distribution of vertex VClusters on induction(top) and collection (bottom) planes for neutrinos (left) and anti-neutrinos (right) for $m u+N p$ events in the fiducial volume. . . . . . . . . . . . . . . . . . . 13

14.18 Distribution of vertex LineMergers on induction(top) and collection (bottom) planes for neutrinos (left) and anti-neutrinos (right) for $m u+N p$ events in the fiducial volume. . . . . . . . . . . . . .

14.19 Distribution of exiting tracks for neutrinos (left) and anti-neutrinos (right) for $m u+\mathrm{Np}$ events in the fiducial volume. . . . . . . . . . 133

14.20 The area normalized distributions of number of vertex VCluster (top left), LineMergers (top right) and number of exiting tracks (bottom) after matching with MINOS for anti-neutrinos. Errors are statistical only. . . . . . .

14.21 Area normalized distribution of number of LineMergers on collection plane for anti-neutrinos after requiring all the automated cuts with the exception of cut on LineMerger and number of exiting tracks . Errors are statistical only. . . . . . . . . . . . . . . . . .

14.22 Area normalized distribution of number of LineMergers on collection plane for anti-neutrinos after requiring all the automated cuts with the exception of number of exiting tracks . Errors are statistical only. . . . . . . . . .

14.23 Area normalized distribution of number of exiting tracks after all the automated cuts for anti-neutrinos. Errors are statistical only. . . . . . . . .

14.24 Kinetic energy versus length for simulated particles. The minimum wire pitch in ArgoNeuT is also shown and the corresponding to it threshold for protons is indicated. 
14.25 Charge in ADC versus proton momentum. The black points correspond to the theory prediction coming from NIST Beta-Bloch equation corrected for recombination effect. The red points come from the ArgoNeuT simulation, where "ADC" refers to the sum of the signal from all proton hits and momentum comes from MC-truth information.

14.26 Charge in ADC versus proton momentum with the added contribution from

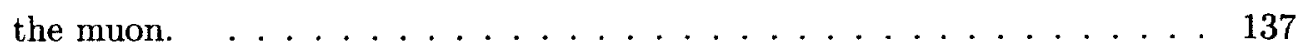

14.27 Scanning tools . . . . . . . . . . . . . . . . . 138

14.28 Example of candidate $\mu+0 \mathrm{p}$ event $\ldots \ldots \ldots \ldots \ldots$

14.29 Example of candidate $\mu+2 \mathrm{p}$ event $\ldots \ldots \ldots \ldots \ldots$

14.30 Example of candidate $\mu+2$ p event $\ldots \ldots \ldots \ldots \ldots$. . . . . . . . 141

14.31 Example of candidate $\mu+3$ p event $\ldots \ldots \ldots \ldots \ldots$. . . . . . . 142

14.32 Composition of sample picked by a scanner for neutrinos in anti-neutrino mode . . . . . . . . . . . . . . . . . . . . . 143

14.33 Composition of sample picked by a scanner for anti-neutrinos in antineutrino mode . . . . . . . . . . . . . . .

14.34 Energy loss per unit track length as a function of residual range (distance to the track end) for different simulated charged particles. . . . . . . . . 146

14.35 Event display showing a single track with no activity at the vertex. $\mathrm{dE} / \mathrm{dx}$ versus residual range plot is shown with the tracks's overlaid hits which lie on top of the muon prediction. The hits do not follow the simulated curve for small values of residual range as this track is not stopping. The slight rise of $\mathrm{dE} / \mathrm{dx}$ along the track seen in the plot is due to delta rays which are visible as more ionizing, red hits in the collection plane. It is categorized as mu+0p event. . . . . . . . . . . . . . . 147

14.36 Event display showing a single track and high ionization at the vertex which is reconstructed as $0.6 \mathrm{~cm}$ long proton track. . . . . . . . . . 148

14.37 Event display showing a muon track with a long proton track. . . . . . . 149

14.38 Event display showing a muon and a reconstructed pion track. . . . . . 150

14.39 Event display showing a muon track accompanied by a proton and a pion track at the vertex . . . . . . . . . . . . . . . . . . . . 151

14.40 Event display showing a muon track with the reconstructed proton track at the vetex. . . . . . . . . . . . . . . . . 
14.41 Confusion matrix for protons, kaon, muons and pions generated by reconstructing simulated particles. It is seen that the efficiency of reconstructing protons is $97 \%, 3 \%$ of the time proton is confused with a kaon. . . . . 155

14.42 Distribution of pion momentum for MC events that passed automated cuts (anti-neutrinos in anti-neutrino mode) . . . . . . . . . . 157

14.43 Examples of events with very low energy pions, on the order of $0.1 \mathrm{GeV} / \mathrm{c} \quad 158$

14.44 The flux for neutrinos in anti-neutrino mode. . . . . . . . . . 159

14.45 The flux for anti-neutrinos in anti-neutrino mode. . . . . . . . . 160

14.46 Distribution of proton multiplicities for neutrinos in the data and comparison with GENIE expectation (top). The error on the data includes both the statistical and systematic uncertainty. The MC band (in grey) represents the large uncertainty coming from the flux. The bottom plot shows the distribution of proton multiplicities as modeled by the $\mathrm{MC}$ with various contributions, no uncertainty is shown for the clarity of the plot. . . . . .

14.47 Distribution of proton multiplicities for anti-neutrinos in the data and comparison with GENIE expectation (top). The error on the data includes both the statistical and systematic uncertainty. The MC band (in grey) represents the large uncertainty coming from the flux. The bottom plot shows the distribution of proton multiplicities as modeled by the MC with various contributions, no uncertainty is shown for the clarity of the plot. . . . .

14.48 Muon angle and momentum distribution for $\mu+\mathrm{Np}$ neutrino events. Data events entering in these plots come after calorimetry reconstruction (background subtracted) and the MC corresponds to contained $\mu+\mathrm{Np}$ after all cuts. . . . . . . . . . . . . . . . . 167

14.49 Muon angle and momentum distribution for $\mu+0 \mathrm{p}$ neutrino events. Data events entering in these plots come after calorimetry reconstruction and the MC corresponds to contained $\mu+0 \mathrm{p}$ after all cuts. . . . . . . . 167

14.50 Muon angle and momentum distribution for $\mu+1 \dot{\mathrm{p}}$ neutrino events. Data events entering in these plots come after calorimetry reconstruction and the $\mathrm{MC}$ corresponds to contained $\mu+1 \mathrm{p}$ after all cuts. . . . . . . 168 
14.51 Muon angle and momentum distribution for $\mu+\mathrm{Np}$ anti-neutrino events. Data events entering in these plots come after calorimetry reconstruction (background subtracted), the $\mathrm{MC}$ corresponds to contained $\mu+\mathrm{Np}$ after all cuts. . . . . . . . . . . . . . . . . . . . 168

14.52 Muon angle and momentum distribution for $\mu+0 \mathrm{p}$ anti-neutrino events. Data events entering in these plots come after calorimetry reconstruction, the MC corresponds to contained $\mu+0$ p after all cuts. . . . . . . . .

14.53 Muon angle and momentum distribution for $\mu+1 \mathrm{p}$ anti-neutrino events. Data events entering in these plots come after calorimetry reconstruction, the $\mathrm{MC}$ corresponds to contained $\mu+1 \mathrm{p}$ after all cuts. . . . . . . .

14.54 The muon momentum distribution for neutrino $\mu+N p$ data events after calorimetric reconstruction (red) and the background events coming from the MC (black). The plots are scaled appropriately to indicate the relative percentage of background. . . . . . . . . . . . . 170

14.55 The muon angular distribution for neutrino $\mu+N p$ data events after calorimetric reconstruction (red) and the background events coming from the $\mathrm{MC}$ (black). The plots are scaled appropriately to indicate the relative percentage of background. . . . . . . . . . . . . . .

14.56 The muon momentum distribution for anti-neutrino $\mu+N p$ data events after calorimetric reconstruction (red) and the background events coming from the MC (black). The plots are scaled appropriately to indicate the relative percentage of background. . . . . . . . . . . .

14.57 The muon angular distribution for anti-neutrino $\mu+N p$ data events after calorimetric reconstruction (red) and the background events coming from the MC (black). The plots are scaled appropriately to indicate the relative percentage of background. . . . . . . . . . . . . . . . 171

14.58 Distribution of proton length, kinetic energy and angle with respect to the beam direction for $\mu+1 \mathrm{p}$ neutrino events. In data this corresponds to sample of $N=1$ events after calorimetry and in the $M C$ to true $\mu+1 p$ neutrino events after all cuts. . . . . . . . . . . . . . . . 173 
14.59 Distribution of proton length, kinetic energy and angle with respect to the beam direction for $\mu+1 p$ anti-neutrino events. In data this corresponds to sample of $\mathrm{N}=1$ events after calorimetry and in the $\mathrm{MC}$ to true $\mu+1 \mathrm{p}$ neutrino events after all cuts. . . . . . . . . . . . . . . . . 174

14.60 Distribution of proton length, kinetic energy and angle with respect to the beam direction for $\mu+2 p$ neutrino events. In data this corresponds to sample of $\mathrm{N}=2$ events after calorimetry and in the $\mathrm{MC}$ to true $\mu+2 \mathrm{p}$ neutrino events after all cuts. . . . . . . . . . . . . . . . 175

14.61 Distribution of proton length, kinetic energy and angle with respect to the beam direction for $\mu+2 p$ anti-neutrino events. In data this corresponds to sample of $\mathrm{N}=2$ events after calorimetry and in the MC to true $\mu+2 \mathrm{p}$ neutrino events after all cuts. . . . . . . . . . . . 176 


\section{List of Tables}

14.1 The reduction percentage of events after VCluster, LineMerger and 3d Exiting Tracks cuts for neutrinos in anti-neutrino mode. . . . . . . . . . . . . 131

14.2 The reduction percentage of events after VCluster, LineMerger and 3d Exiting Tracks cuts for antineutrinos in anti-neutrino mode. . . . . . . . . 132

14.3 Number of $\mu+\mathrm{Np}$ data events after scanning and calorimetry for neutrinos in anti-neutrino mode. . . . . . . . . . . . . . . 146

14.4 Number of $\mu+\mathrm{Np}$ data events after scanning and calorimetry for antineutrinos in anti-neutrino mode. . . . . . . . . . . . 146

14.5 Percentage of lost events after each sets of cuts for neutrinos in anti-neutrino mode. . . . . . . . . . . . . . . . . . . . . . . 153

14.6 Percentage of lost events after each sets of cuts for anti-neutrinos in antineutrino mode. . . . . . . . . . . . . . . . . . . . . . 153

14.7 Efficiency of cuts, $\epsilon_{\text {cuts }}^{N}$ for $\mu+\mathrm{Np}$ neutrino events for each multiplicity $N$. The term 'inclusive' refers to $\mu+\mathrm{Np}$ events without the restriction on proton containment. . . . . . . . . . . . . . . . . . . 154

14.8 Efficiency of scanning, $\epsilon_{\text {scan }}^{N}$ for $\mu+N p$ neutrino events for each multiplicity $\mathrm{N}$. The second column shows efficiency of scanning for inclusive events (no restriction on proton containment) and the third shows it for sample with contained protons only. It is seen that the efficiency for contained protons is very high, while it is lower for inclusive sample. This behaviour is expected, as a scanner is supposed to pick out events with contained protons and thus the efficancy of this class of events is expected to be higher. 
14.9 Efficiency of cuts, $\epsilon_{c u t s}^{N}$ for $\mu+\mathrm{Np}$ anti-neutrino events for each multiplicity $\mathrm{N}$. The term 'inclusive' refers to $\mu+\mathrm{Np}$ events without the restriction on proton containment. . . . . . . . . . . . . . 155

14.10 Efficiency of scanning, $\epsilon_{s c a n}^{N}$, for $\mu+N$ p anti-neutrino events for each multiplicity N. It is seen that the efficiency for contained protons is very high, while it is lower for inclusive sample. This behaviour is expected, as a scanner is supposed to pick out events with contained protons and thus the efficancy of this class of events is expected to be higher. . . . . . . . . 155

14.11 Number of background events for $\mu+\mathrm{Np}$ sample of neutrinos in anti-neutrino mode. . . . . . . . . . . . . . . . . . . . 156

14.12 Number of background events for $\mu+\mathrm{Np}$ sample of anti-neutrinos in antineutrino mode. . . . . . . . . . . . . . . . . 156

14.13 Data comparison with GENIE in terms of proton multiplicity of $\mu+\mathrm{Np}$ events and fractions of events for neutrinos in anti-neutrino mode. The first number following the " \pm " represents the statistical error and the second one the systematic error.

14.14 Data comparison with GENIE in terms of proton multiplicity of $\mu+\mathrm{Np}$ events and fractions of events for anti-neutrinos in anti-neutrino mode. The first number following the " \pm " represents the statistical error and the second one the systematic error. . . . . . . . . . . . . . . . . 162

14.15 Fractions of non-CCQE events as a function of proton multiplicity in $\mu+\mathrm{Np}$ events for neutrinos and anti-neutrinos in anti-neutrino mode. . . . . . 162

14.16 Total statistical fractional errors for neutrinos and anti-neutrinos. . . . . 165

14.17 Total fractional errors for neutrinos and anti-neutrinos obtained by adding systematic error with all systematic in quadrature. . . . . . . . . . . 166

14.18 Fractions of backward going protons in the DATA and GENIE for N=1,2 multiplicity for neutrinos and anti-neutrinos. . . . . . . . . . . 177 


\section{Introduction to the Dissertation}

The thesis presented here describes the first topological measurement of charged current muon-neutrino $\mu+N p$, muon and any number of protons at a vertex, interactions in neutrino physics. The measurement is possible due to the use of the superb Liquid Argon Time Projection Chamber (LArTPC) technology of the ArgoNeuT experiment, which provides bubble-like-chamber images of neutrino interactions. The measurement takes full advantage of this technology by reconstructing proton kinematics with a very low energy threshold. This is the first time in neutrino physics that we are able to address this aspect of neutrino interactions. In addition to illustrating the power of this technique, the measurement is a stepping stone for exploration of the signal events for neutrino oscillations in LArTPC detectors. The analysis here makes a strong case for studying neutrino interactions based on a topological measurement and avoiding the reliance on the $\mathrm{MC}$ as much as possible. The significant impact of nuclear effects in neutrino interactions will be shown making it clear that it cannot be ignored. Furthermore, the exploration of $\mu+N p$ interactions presented here can be used to tune Monte Carlo generators as it offers insight into the details of vertex activity in neutrino interactions.

The dissertation is divided into five parts. It begins with the proposal of the neutrino, neutrino detection and neutrino sources in Chapter 2. Chapter 3 describes the phenomenon of neutrino oscillation with the experimental evidence and finishes with a summary of still unanswered questions. Chapter 4 explores neutrino scattering and its importance. Part II of the thesis focuses on neutrino detection with the LArTPC technology using ArgoNeuT as an example. Chapter 5 serves to introduce the LArTPC concept, Chapter 6 summarizes event imagining and display with ArgoNeuT. Chapter 7 closes this part with the vision of the future of the LAr technology in the US. Part III is dedicated to the ArgoNeuT 
experiment description. It begins with the NuMI neutrino beam description in Chapter 8 . Chapter 9 summarizes the ArgoNeuT hardware. Chapter 10 describes commissioning and data taking. Chapter 11 gives an overview of the detector performance. Part IV focuses on two very important aspects for any measurement. It begins with a description of the ArgoNeuT MC simulation given in Chapter 12. Chapter 13 describes the LArSoft software used for reconstruction of events. Part $\mathrm{V}$ presents the analysis of the data including the importance of the described here measurement and the results. 


\section{PART I}

Description of the Neutrino 


\section{Neutrino Overview}

\subsection{The Birth of the Neutrino}

Neutrinos were postulated to exist as a consequence of the study of nuclear beta decay. In this process a radioactive nucleus is transformed into a slightly lighter nucleus accompanied by electron emission:

$$
N(A, Z) \rightarrow N^{\prime}(A, Z+1)+e^{-}
$$

The process was initially assumed to be a two body disintegration since only the electron and the recoiling daughter nucleus were observed. Conservation of energy and momentum for the observed particles required the electron energy to be:

$$
E_{e}=\frac{m_{N}^{2}-m_{N^{\prime}}^{2}+m_{e}^{2}}{2 m_{N}}
$$

Thus, the energy of emitted electron is expected to have a unique value. However, a continuous spectrum of energy was observed experimentally with the endpoint given by Equation 2.2. This observation threatened the survival of one of the most cherished conservation laws in physics. Wolfgang Pauli suggested, however, that an additional particle is emitted in the beta decay [1]. He proposed the particle was neutral to conserve the charge, carrying spin angular momentum of $1 / 2$ and called it 'neutron'. By the time he published it in 1933 the neutron had been discovered by Chadwick and Fermi renamed it as 'neutrino' [2] . Today we know that it was actually antineutrino that was emitted in the beta decay and we can write the fundamental interaction in correct terminology as:

$$
n \rightarrow p+e^{-}+\bar{\nu}_{e}
$$


In 1934 Fermi laid out the theory of weak interactions and successfully explained the experimental observations [3]. Despite the strong theoretical evidence for the existence of neutrinos they were not detected until 1956 . Neutrinos interact very weakly with matter and thus detecting them is a challenge.

\subsection{Detection of the Neutrino}

The electron anti-neutrino was first detected in 1956 by Cowan and Reines at the Savannah River nuclear reactor [4]. The experiment consisted of two tanks of 200 liters of water each with dissolved Cadmium Chloride. The tanks were sandwiched between three scintillator detectors. The proton in the water provided target for the interaction known as inverse beta decay:

$$
\bar{\nu}_{e}+p \rightarrow e^{+}+n
$$

The annihilation of $e^{+}$with $e^{-}$produced gamma rays, which could be detected due to the scintillator and photomultiplier tubes. The neutron was absorbed in cadmium also providing a gamma ray few microseconds after the first signal, $n^{48} C d \rightarrow \gamma^{49} C d$. The detected double gamma ray signal was correlated with the reactor being on. The Nobel prized was presented in 1996 for this great discovery.

A second flavor of neutrino, $\nu_{\mu}$, was discovered in 1962 at Brookhaven [5]. It was achieved by detecting muons from muon neutrino interactions. It was the first experiment, which we now categorize as accelerator neutrino experiment. The fact that muons instead of electrons were detected proved the existence of a second neutrino flavor. The third neutrino flavor, $\nu_{\tau}$, was discovered at Fermilab in 2000 [6].

\subsection{Brief Description of the Neutrino}

Today we have verified that there are three types or flavours of neutrinos that interact via the weak interaction: $\nu_{e}, \nu_{\mu}, \nu_{\tau}$ and their antiparticles. Weak interactions are mediated by exchange of $W^{ \pm}$or the $Z^{0}$ boson, referred to as charged current (CC) and neutral current (NC) interactions, respectively. When a $W$ is emitted, charge conservation at the vertex demands that a charged lepton exits the interaction. The flavour of the incoming neutrino is tagged by the charged partner, which exits the $\mathrm{CC}$ interaction. For example, a scattered 
electron implies a $\nu_{e}$ interaction. The neutrino always emits the $W^{+}$while the antineutrino always emits the $W^{-}$in the CC interaction. The charge of the $W$ is governed by the charge conservation of the outgoing lepton.

The width of the $Z^{0}$ boson, measured at LEP [7], limits the number of weakly interacting neutrino flavours to three. Other neutrino candidates, such as a possible "sterile neutrino" cannot interact weakly and are allowed by some beyond the Standard Model theories. Neutrinos have half-integer spin and are electrically neutral. Until recently the neutrinos were thought to be massless. However, the discovery of neutrino oscillation proved otherwise. The mixing between flavours and the implications for mass will be discussed in Chapter 3. As described in Section 2.3.1, neutrinos also have a specific handedness: neutrinos are left-handed while anti-neutrinos are right-handed.

\subsubsection{Parity Violation and Handedness}

Until the late 1950s, it was thought that parity was a symmetry of all fundamental interactions. The experiment that showed that parity is violated in weak interactions made use of the $\beta$ decay of the polarized ${ }^{60} \mathrm{Co}$ and was performed by $\mathrm{Wu}[8]$.

$$
{ }^{60} \mathrm{Co} \rightarrow{ }^{60} \mathrm{Ni}+e^{-}+\bar{\nu}_{e}
$$

The nuclear spins of a crystal of cobalt salt were polarized by applying a strong magnetic field. The measurement of the angular distribution of the emitted electrons, $\theta_{e}$, was performed. It was found that the electrons were emitted preferentially in a direction opposite to the field, meaning opposite to the spin direction of the cobalt nuclei. In other words, the expectation value of $\cos \theta_{e}$ was negative. However, under parity transformation the expectation value changes sign:

$$
\left\langle\cos \theta_{e}\right\rangle=\left\langle\frac{\vec{s} \cdot \vec{p}}{|\vec{s}||\vec{p}|}\right\rangle \stackrel{P}{\rightarrow}\left\langle\frac{\vec{s} \cdot-(\vec{p})}{|\vec{s}||\vec{p}|}\right\rangle=-\left\langle\cos \theta_{e}\right\rangle
$$

where $\vec{s}$ denotes the spin of the cobalt and $\vec{p}$ the momentum of the emitted electron. If parity is conserved, equal numbers of electrons should be emitted parallel and antiparallel to the magnetic field. However, the obtained result implied that the parity is violated. It is not limited to beta decay in cobalt but as it turned out, the parity violation is the signature of the weak force. 
For a spin 1/2 Dirac particle, helicity is defined as the projection of a particle's spin $\mathbf{S}$ along its direction of motion $\hat{\mathbf{p}}$ with operator $\mathbf{S} \cdot \hat{\mathbf{p}}$. For this particle two outcomes are possible: spin aligned along the direction of motion ("right helicity") or spin aligned opposite the direction of motion ("left helicity"). Helicity is not Lorentz-invariant and thus its sign is frame dependent for massive particles. It is possible to boost to a frame moving faster than the particle and as result flip the sign of momentum but leaving the same spin direction. For massless particles the helicity is Lorentz-invariant.

The quantity which is frame-independent for both massive and massless Dirac particles is handedness (chirality). The two distinguished states are "left-handed" and "right-handed". Helicity and handedness are the same for massless particles. Massive fermions can have both right-handed $(\mathrm{RH})$ and left-handed $(\mathrm{LH})$ components. Thus, a helicity eigenstate is a combination of handedness states.

It was discovered that neutrinos are left-handed while anti-neutrinos are right-handed. The handedness could be studied using an indirect method of the pion decay, $\pi^{-} \rightarrow \mu^{-}+\bar{\nu}_{e}$. For pion at rest, the muon and the neutrino come out back to back. Since pion has spin 0 , the spins of the products are oppositely aligned. Thus, if the anti-neutrino is right handed, the muon is as well and this is what is found experimentally. Similarly, in the $\pi^{+}$decay the anti-muon is always left-handed indicating that the neutrino is left-handed.

\subsection{Neutrino Sources}

Neutrinos observed on earth come from the astrophysical sources such as the Sun, cosmic ray interactions in the upper atmosphere and terrestrial sources such as reactors and accelerators. The range of energies covered by neutrinos and their sources is highlighted in Figure 2.1.

The sources in the few $\mathrm{MeV}$ range are the Sun and reactors. The interaction taking place in the Sun that produce the neutrinos are shown in Table 2.2. The flux consists of pure $\nu_{e}$ 's. The reactors, on the other hand, produce a nearly pure $\bar{\nu}_{e}$ flux and the energy peaks from about 3 to $7 \mathrm{MeV}$.

Higher energy neutrinos are produced in the atmosphere and at accelerators. These neutrinos are mainly produced through meson decays. In the case of the atmospheric neutrinos, the cosmic rays hit atmospheric nuclei producing a shower of mesons. The mesons may decay to neutrinos as they travel through the atmosphere to Earth. In the 


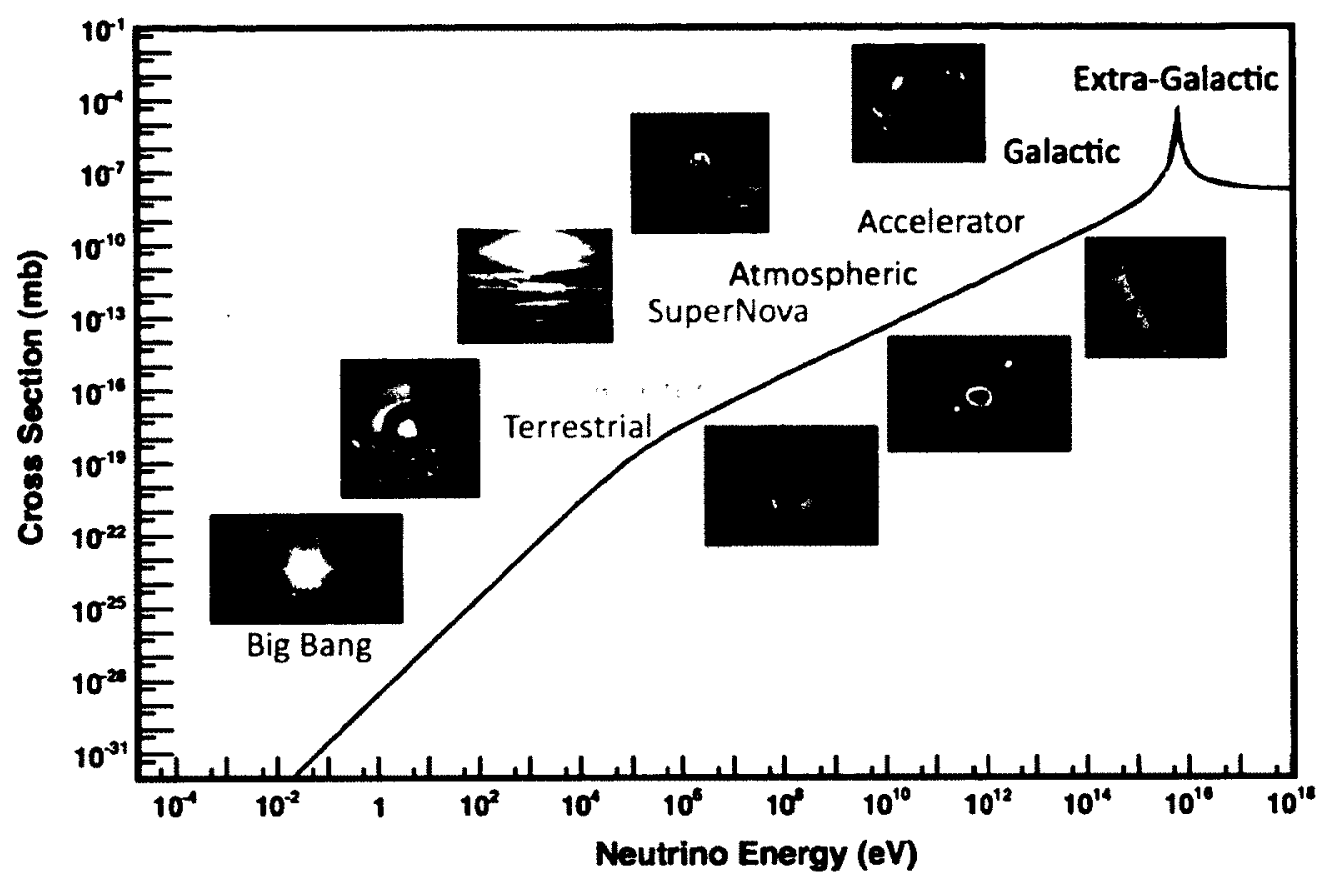

Figure 2.1: Neutrino sources as a function of energy. The electroweak cross-section for $\bar{\nu}_{e} e^{-} \rightarrow \bar{\nu}_{e} e^{-}$ is shown for comparison. (from Reference [60])

\begin{tabular}{c|c}
\hline Common Terminology & Reaction \\
\hline "pp neutrinos" & $p+p \rightarrow^{2} \mathrm{H}+e^{-}+\nu_{e}$ \\
"pep neutrinos" & $p+e^{-}+p \rightarrow^{2} \mathrm{H}+\nu_{e}$ \\
"7 Be neutrinos" & ${ }^{7} \mathrm{Be}+e^{-} \rightarrow^{7} \mathrm{Li}+\nu_{e}$ \\
"8 $\mathrm{B}$ neutrinos" & ${ }^{8} \mathrm{~B} \rightarrow{ }^{8} \mathrm{Be}^{*}+e^{+}+\nu_{e}$ \\
"hep neutrinos" & ${ }^{3} \mathrm{He}+p \rightarrow^{4} \mathrm{He}+e^{+}+\nu_{e}$ \\
\hline
\end{tabular}

Figure 2.2: Sources of neutrinos from the Sun.

case of an accelerator beam, protons struck a target producing secondary mesons. The mesons can then be focused by the use of magnets called horns, which are sign-selecting. The beamline is equipped with a long decay region followed by a beam dump and a region of dirt to remove all particles except neutrinos. These sources are predominantly composed of

\begin{tabular}{c|c}
\hline 2-body pion decay & $\pi^{+} \rightarrow \mu^{+} \nu_{\mu}, \pi^{-} \rightarrow \mu^{-} \bar{\nu}_{\mu}$ \\
2-body kaon decay & $K^{+} \rightarrow \mu^{+} \nu_{\mu}, K^{-} \rightarrow \mu^{-} \bar{\nu}_{\mu}$ \\
muon decay & $\mu^{+} \rightarrow e^{+} \bar{\nu}_{\mu} \nu_{e}, \mu^{-} \rightarrow e^{-} \nu_{\mu} \bar{\nu}_{e}$ \\
$K_{e 3}$ decay & $K^{+} \rightarrow \pi^{0} e^{+} \nu_{e}, K^{-} \rightarrow \pi^{0} e^{-} \bar{\nu}_{e}, K^{0} \rightarrow \pi^{-} e^{+} \nu_{e}, K^{0} \rightarrow \pi^{+} e^{-} \bar{\nu}_{e}$ \\
\hline
\end{tabular}

Figure 2.3: Sources of neutrinos in atmospheric and accelerator experiments. 
$\nu_{\mu}$ 's. The main sources of neutrinos in atmospheric and accelerator experiments are shown in Table 2.3. 


\section{Neutrino Oscillation}

There exists convincing evidence that atmospheric, solar, reactor, and accelerator neutrinos change from one flavour to another. The neutrino flavour change implies that neutrinos have masses and that leptons mix. A summary of formalism of the neutrino oscillation is given below. A review of experimental results confirming the oscillations will also be presented.

\subsection{Neutrino Oscillation in Vacuum}

The existence of neutrino masses means that there is a spectrum of neutrino mass eigenstates $\nu_{i}$ where $i=1,2, \ldots$ each having a mass $m_{i}$. The leptonic mixing can be understood by studying the decay of $\mathrm{W}$ boson: $W^{+} \rightarrow \nu_{i}+\bar{l}_{\alpha}$ where $\alpha=\mathrm{e}, \mu$, or $\tau$, and $l_{e}$ is understood to be the electron, $l_{\mu}$ the muon, and $l_{\tau}$ the tau. Mixing means that the neutrino mass eigenstate, $\nu_{i}$, in the $W^{+}$decay is not always the same $\nu_{i}$. If the amplitude for $W^{+}$decay to produce $\nu_{i}+\bar{l}_{\alpha}$ is denoted by $U_{\alpha i}^{*}$ then the neutrino state emitted with $\bar{l}_{\alpha}$ is :

$$
\left|\nu_{\alpha}>=\sum_{i} U_{\alpha i}^{*}\right| \nu_{i}>
$$

The quantities $U_{\alpha i}$ can be written in a matrix form known as the leptonic mixing matrix. In the three neutrino case $U$ can be written as

$$
U=\left[\begin{array}{ccc}
U_{e 1} & U_{e 2} & U_{e 3} \\
U_{\mu 1} & U_{\mu 2} & U_{\mu 3} \\
U_{\tau 1} & U_{\tau 2} & U_{\tau 3}
\end{array}\right]
$$


In the Standard Model this matrix is unitary, meaning that when a neutrino interacts in a detector and produces a charged lepton, this lepton will be of the same flavour as the neutrino creating it. Expression 3.1 can be rewritten to express each mass eigenstate, $\nu_{i}$ as a superposition of flavours, instead of expressing a neutrino of definite flavour as a superposition of mass eigenstates, as:

$$
\left|\nu_{i}>=\sum_{\alpha} U_{\alpha i}\right| \nu_{\alpha}>
$$

The flavour fraction is $\left|U_{\alpha i}\right|^{2}$ and is a probability that a charged lepton will be of a certain flavour when $\nu_{i}$ interacts in a detector and creates it.

A neutrino flavour change probability will be derived following reference [9]. The probability can be derived by starting with a neutrino source that produces a neutrino of flavour $\alpha$ and a charged lepton $\bar{l}_{\alpha}$. The neutrino then travels to a detector where it interacts with a target and produces a charged lepton of flavour $\beta$. Meaning that when the neutrino interacted it was of flavour $\beta, \nu_{\beta}$. During its travel, the neutrino evolved from $\nu_{\alpha}$ to $\nu_{\beta}$ assuming $\alpha \neq \beta$. This change of flavour is a quantum-mechanical effect called oscillation.

The amplitude of neutrino oscillation $\nu_{\alpha} \rightarrow \nu_{\beta}$ is a product of three factors:

1. The amplitude for the neutrino produced together with an $\bar{l}_{\alpha}$ by the source to be a $\nu_{i}$. As noted earlier this amplitude is $U_{\alpha i}^{*}$.

2. The amplitude for the produced $\nu_{i}$ to propagate from the source to the detector, denoted by $\operatorname{Prop}\left(\nu_{i}\right)$.

3. The amplitude for the lepton produced by the $\nu_{i}$ to be a $l_{\beta}$ when the neutrino interacts in the detector. From the Hermiticity of the Hamiltonian this amplitude is $\operatorname{Amp}\left(\nu_{i} \rightarrow\right.$ $\left.l_{\beta} W\right)=U_{\beta i}\left(\right.$ since $\left.\operatorname{Amp}\left(W \rightarrow \bar{l}_{\alpha} \nu_{i}\right)=U_{\alpha i}^{*}\right)$.

Putting it all together, the amplitude of neutrino oscillation from flavour $\alpha$ to flavour $\beta$ is:

$$
A m p\left(\nu_{\alpha} \rightarrow \nu_{\beta}\right)=\sum_{i} U_{\alpha i}^{*} \operatorname{Prop}\left(\nu_{i}\right) U_{\beta i}
$$


In order to understand how a neutrino born as the $\nu_{\alpha}$ evolves in time, a Schrodinger's equation is applied to the $\nu_{i}$ component of $\nu_{\alpha}$ and one obtains:

$$
\left|\nu_{i}\left(\tau_{i}\right)>=e^{-i m_{i} \tau_{i}}\right| \nu_{i}(0)>
$$

where $m_{i}$ is the mass of $\nu_{i}$, and $\tau_{i}$ is time in the $\nu_{i}$ frame. The Lorentz-invariant phase factor can be expressed in terms of the time, $t$, and distance, $L$, that the neutrino travels between its source and the detector. $\mathrm{L}$ and $\mathrm{t}$ are chosen by each experiment and are common to all $\nu_{i}$ components of the beam. Other laboratory-frame variables are the energy, $E_{i}$, and momentum, $p_{i}$ of mass eigenstate $\nu_{i}$. Thus, the phase factor can be written as:

$$
e^{-i m_{i} \tau_{i}}=e^{-i\left(E_{i} t-p_{i} L\right)}
$$

The different mass eigenstate components of a beam that contribute coherently to the oscillation signal must have the same energy. Considering the tiny neutrino masses, the momentum, $p_{i}$, can be written as:

$$
p_{i}=\sqrt{E^{2}-m_{i}^{2}} \cong E-\frac{m_{i}^{2}}{2 E}
$$

Plugging this result into the phase factor, one obtains:

$$
m_{i} \tau_{i} \cong E(t-L)+\frac{m_{i}^{2}}{2 E} L
$$

The factor $E(t-L)$ is common to all interfering mass eigenstates and can be ignored. Now, the amplitude for the produced $\nu_{i}$ to propagate from the source to the detector can be written as:

$$
\operatorname{Prop}\left(\nu_{i}\right)=e^{-i m_{i}^{2} \frac{L}{2 E}}
$$

Putting all three amplitudes together, the amplitude for a neutrino to oscillate from flavour $\alpha$ to flavour $\beta$ while traveling a distance $L$ through vacuum with energy $E$ is given by

$$
A m p\left(\nu_{\alpha} \rightarrow \nu_{\beta}\right)=\sum_{i} U_{\alpha i}^{*} e^{-i m_{i}^{2} \frac{L}{2 E}} U_{\beta i}
$$

Finally, the probability of oscillation for $\nu_{\alpha} \rightarrow \nu_{\beta}$ is acquired by squaring the above expression: 


$$
\begin{aligned}
P\left(\nu_{\alpha} \rightarrow \nu_{\beta}\right)=\left|A m p\left(\nu_{\alpha} \rightarrow \nu_{\beta}\right)\right|^{2}=\delta_{\alpha \beta}- & 4 \sum_{i>j} \Re\left(U_{\alpha i}^{*} U_{\beta i} U_{\alpha j} U_{\beta j}^{*}\right) \sin ^{2}\left(\Delta m_{i j}^{2} \frac{L}{4 E}\right) \\
& +2 \sum_{i>j} \Im\left(U_{\alpha i}^{*} U_{\beta i} U_{\alpha j} U_{\beta j}^{*}\right) \sin \left(\Delta m_{i j}^{2} \frac{L}{4 E}\right)
\end{aligned}
$$

where $\Delta m_{i j}^{2} \equiv m_{i}^{2}-m_{j}^{2}$.

The above equation holds for neutrinos. In order to obtain it for anti-neutrinos, one first assumes CPT invariance and obtains:

$$
P\left(\bar{\nu}_{\alpha} \rightarrow \bar{\nu}_{\beta}\right)=P\left(\nu_{\beta} \rightarrow \nu_{\alpha}\right)
$$

By making use of Equation 3.11 one can write:

$$
P\left(\nu_{\beta} \rightarrow \nu_{\alpha} ; U\right)=P\left(\nu_{\alpha} \rightarrow \nu_{\beta} ; U^{*}\right)
$$

And one can write the oscillation probability relation between neutrinos and antineutrinos as

$$
P\left(\bar{\nu}_{\alpha} \rightarrow \bar{\nu}_{\beta} ; U\right)=P\left(\nu_{\alpha} \rightarrow \nu_{\beta} ; U^{*}\right)
$$

Thus, the probability of anti-neutrino oscillation is the same as that for neutrino with the exception of the replacement of the mixing matrix $U$ by its complex conjugate. The last term of Equation 3.11 will be negative for anti-neutrinos if $U$ is not real. The difference in the two probabilities would indicate a CP violation.

\subsubsection{Features of Neutrino Oscillation}

The observation that neutrinos change flavour as they travel implies that they posses mass. If one assumes neutrinos to be massless, all $\Delta m_{i j}^{2}=0$, and then Equation 3.11 leads to $P\left(\nu_{\alpha} \rightarrow \nu_{\beta}\right)=\delta_{\alpha \beta}$. Furthermore, neutrino oscillation implies that $U$ is not diagonal, in other words, there is leptonic mixing.

Inserting the so far omitted factors of $\hbar$ and $\mathrm{c}$ into quantity $\Delta m_{i j}^{2} \frac{L}{4 E}$ from Equation 3.11 leads to:

$$
\Delta m_{i j}^{2} \frac{L}{4 E}=1.27 \Delta m_{i j}^{2}\left(e V^{2}\right) \frac{L(k m)}{E(G e V)}
$$


The factor $\sin ^{2}\left(\Delta m_{i j}^{2} \frac{L}{4 E}\right)$ becomes $\sin ^{2}\left(1.27 \Delta m_{i j}^{2}\left(\mathrm{eV}^{2}\right) \frac{L(\mathrm{~km})}{E(\mathrm{GeV})}\right)$ and is appreciable when the argument is greater than unity. Thus, an experiment's sensitivity to the squared-mass splittings is $\Delta m_{i j}^{2}\left(\mathrm{eV}^{2}\right) \geq E(\mathrm{GeV}) / L(\mathrm{~km})$. In atmospheric oscillation experiments, if one assumes a typical value of $\mathrm{E} \sim 1 \mathrm{GeV}$ and $\mathrm{L} \sim 10000 \mathrm{~km}$ then sensitivity to $\Delta m_{i j}^{2}>10^{-4} \mathrm{eV}^{2}$. It shows that oscillation experiments can be sensitive to very small mass splittings by the right choice of $\mathrm{L} / \mathrm{E}$. However, these experiments are not sensitive to the individual masses.

\subsubsection{Two neutrino mixing}

The best known special case of Equation 3.11 describes oscillation when only two mass eigenstates are relevant. It is important as it is an accurate description for many experiments. If one calls mass eigenstates as $\nu_{1}$ and $\nu_{2}$, and the two neutrinos of definite flavour as $\nu_{\alpha}$ and $\nu_{\beta}$ then there is only one mass splitting $\Delta m^{2}=m_{2}^{2}-m_{1}^{2}$. The mixing matrix is then $2 \times 2$ and can be written as

$$
U=\left[\begin{array}{ll}
U_{\alpha 1} & U_{\alpha 2} \\
U_{\beta 1} & U_{\beta 2}
\end{array}\right]=\left[\begin{array}{cc}
\cos \theta & \sin \theta \\
-\sin \theta & \cos \theta
\end{array}\right]
$$

where $\theta$ is the mixing angle. Inserting the mixing matrix and the splitting into Equation 3.11 one obtains probability of oscillation for two (anti)neutrinos in a much more simplified form for $\beta \neq \alpha$

$$
P\left(\nu_{\alpha} \rightarrow \nu_{\beta}\right)=\sin ^{2} 2 \theta \sin ^{2}\left(\Delta m^{2} \frac{L}{4 E}\right)
$$

\subsubsection{Three neutrino mixing}

As we presently know, there are three neutrinos. However, the existence of sterile neutrinos, that do not coup̉le to the Standard Model W boson, has not been ruled out. The three generation matrix called MNS (Maki-Nakagawa-Sakata) is

$$
U=\left[\begin{array}{ccc}
U_{e 1} & U_{e 2} & U_{e 3} \\
U_{\mu 1} & U_{\mu 2} & U_{\mu 3} \\
U_{\tau 1} & U_{\tau 2} & U_{\tau 3}
\end{array}\right]
$$




$$
\begin{aligned}
& =\left[\begin{array}{ccc}
1 & 0 & 0 \\
0 & c_{23} & s_{23} \\
0 & -s_{23} & c_{23}
\end{array}\right]\left[\begin{array}{ccc}
c_{13} & 0 & s_{13} e^{-i \delta} \\
0 & 1 & 0 \\
-s_{13} e^{i \delta} & 0 & c_{13}
\end{array}\right]\left[\begin{array}{ccc}
c_{12} & s_{12} & 0 \\
-s_{12} & c_{12} & 0 \\
0 & 0 & 1
\end{array}\right]\left[\begin{array}{ccc}
e^{i \alpha_{1} / 2} & 0 & 0 \\
0 & e^{i \alpha_{2} / 2} & 0 \\
0 & 0 & 1
\end{array}\right] \\
& =\left[\begin{array}{ccc}
c_{12} c_{13} \\
-s_{12} c_{23}-c_{12} s_{23} s_{13} e^{i \delta} & c_{12} c_{23}-s_{12} s_{23} s_{13} e^{i \delta} & s_{23} c_{13} \\
s_{12} s_{23}-c_{12} c_{23} s_{13} e^{i \delta} & -c_{12} s_{23}-s_{12} c_{23} s_{13} e^{i \delta} & c_{23} c_{13}
\end{array}\right]\left[\begin{array}{ccc}
e^{i \alpha_{1} / 2} & 0 & 0 \\
0 & e^{i \alpha_{2} / 2} & 0 \\
0 & 0 & 1
\end{array}\right],
\end{aligned}
$$

where $\mathrm{c}$ and $\mathrm{s}$ refer to the sines and cosines of the three mixing angles $\left(\theta_{12}, \theta_{23}\right.$, and $\left.\theta_{13}\right)$ and $\delta, \alpha_{1}$, and $\alpha_{2}$ are $\mathrm{CP}$ violating phases. With three generations of neutrinos, there are three mass splittings $\Delta m_{12}^{2}, \Delta m_{23}^{2}$, and $\Delta m_{13}^{2}$, but only two are independent since $\Delta m_{12}^{2}+\Delta m_{23}^{2}+\Delta m_{31}^{2}=0$.

\subsection{Neutrino Oscillation in Matter}

Many neutrino flavour change experiments involve neutrinos that travel through matter. Interaction of neutrinos with electrons in matter can modify the flavour content of the beam as compared to the vacuum case. Muon neutrinos produced in the solar core can only interact via exchange of the $Z^{0}$ boson, the neutral current interaction. Electron neutrinos, however, can interact via charged current and neutral currents. The scattering by electrons via $\mathrm{W}$ exchange gives rise to an extra interaction potential energy, $V_{W}$. This extra energy is proportional to the number of electrons per unit volume, $N_{e}$, and the Fermi coupling constant, $G_{F}$. The interaction potential energy is given by

$$
V_{W}=+\sqrt{2} G_{F} N_{e}
$$

for electron neutrinos, it has the opposite sign for electron anti-neutrinos.

A neutrino can also exchange a $\mathrm{Z}$ boson with an electron, proton, or neutron. According to the Standard Model any neutrino flavour can participate in this interaction and the amplitude for this $\mathrm{Z}$ exchange is independent of the neutrino flavour. $\mathrm{Z}$ couplings to electron and proton are equal and opposite at zero momentum transfer and will cancel out. The extra interaction potential energy for neutral current, $V_{Z}$, will depend on the number of 
neutrons per unit volume, $N_{n}$. According to Standard Model this potential is

$$
V_{Z}=-\frac{\sqrt{2}}{2} G_{F} N_{e}
$$

for neutrinos, and it has the opposite sign for anti-neutrinos.

It should be noted that the Standard Model interactions do not change neutrino flavour. In other words, neutrino oscillation implies neutrino mass and mixing even when the neutrinos are passing through matter. One can study the differences between the vacuum and the matter oscillations through the use of the Hamiltonian. In the case of neutrino flavour change in vacuum the Hamiltonian can be written as

$$
H_{v a c}=\frac{\Delta m^{2}}{4 E}\left[\begin{array}{cc}
-\cos 2 \theta & \sin 2 \theta \\
\sin 2 \theta & \cos 2 \theta
\end{array}\right]
$$

For the neutrino oscillation in matter, the Hamiltonian must be modified and is given by

$$
H_{M}=H_{v a c}+V_{W}\left[\begin{array}{ll}
1 & 0 \\
0 & 0
\end{array}\right]+V_{W}\left[\begin{array}{ll}
1 & 0 \\
0 & 1
\end{array}\right]
$$

The second term on the right represents the contribution from the interaction potential cause by $\mathrm{W}$ exchange. As noted earlier, this contribution affects only $\nu_{e}$ so only the upper left element of $H_{M}$ in non-vanishing. The last term represents the contribution from the $\mathrm{Z}$ exchange. This interaction potential affects all flavours equally and has a form of the identity matrix, and can be dropped.

If one defines

$$
\begin{gathered}
\Delta m_{M}^{2}=\Delta m^{2} \sqrt{\sin ^{2} 2 \theta+(\cos 2 \theta-x)^{2}} \\
\sin ^{2} 2 \theta_{M}=\frac{\sin ^{2} 2 \theta}{\sin ^{2} 2 \theta+(\cos 2 \theta-x)^{2}}
\end{gathered}
$$

where

$$
x=\frac{2 \sqrt{2} G_{F} N_{e} E}{\Delta m^{2}}
$$

Then the Hamiltonian in matter can be written as

$$
H_{M}=\frac{\Delta m_{M}^{2}}{4 E}\left[\begin{array}{cc}
-\cos 2 \theta_{M} & \sin 2 \theta_{M} \\
\sin 2 \theta_{M} & \cos 2 \theta_{M}
\end{array}\right]
$$


Comparing $H_{M}$ with $H_{v a c}$ ones sees that they are the same with the exception of $\Delta m^{2}$ now being $\Delta m_{M}^{2}$, and $\theta$ being replaced by $\theta_{M}$. Thus, the splitting between the effective squared-masses of these eigenstates in matter is different from the vacuum, and the effective mixing angle in matter is different from the vacuum mixing angle $\theta$. Similarly, the oscillation probability in matter is the same as in vacuum, except for the replacement of the vacuum parameters $\theta$ and $\Delta m^{2}$ by their equivalents in matter.

The quantity $\mathrm{x}$, given in Equation 3.25, indicates the fractional importance of the matter effect on oscillation involving vacuum mass splitting $\Delta m^{2}$. The numerator represents the extra energy of a $\nu_{e}$ due to matter interactions. The matter effect increases with neutrino energy $\mathrm{E}$ and is sensitive to the sign of $\Delta m^{2}$. Thus, it can be used to determine whether $\nu_{2}$ or $\nu_{1}$ is heavier. The sign of the potential is different for neutrinos and antineutrinos as noted earlier. This can give rise to a difference between the oscillation in matter for neutrinos and anti-neutrinos. Thus, oscillation experiments must disentangle whether the difference comes from the matter effects or from a mixing matrix $U$ that is not real.

\subsection{Adding Mass Term to the Standard Model}

One of the greatest advances in particle physics over the past decade has been the discovery of neutrino mass and mixing. In the Standard Model there are no right-handed neutrinos, $\nu_{R}$. Thus, neutrinos are massless in the Standard Model distinguished only by lepton numbers. A way to realize neutrino masses in the Standard Model is through a "Dirac mass term". It can take the following form

$$
\mathcal{L}_{D}=-m_{D} \bar{\nu}_{L} \nu_{R}+\text { h.c. }
$$

where $m_{D}$ is a constant and h.c. is the hermitian conjugate. The right-handed neutrino field, $\nu_{R}$, was added to the Standard Model in order to construct the Dirac mass term. This mass term conserves the total lepton number $\mathrm{L}$.

Another possibility is to construct "Majorana mass term". This term would violate total lepton number $\mathrm{L}$ conservation, so the neutrino is its own anti-particle. The Majorana mass term can be made with only left-handed neutrinos, only right-handed neutrinos, or their combination. For example

$$
\mathcal{L}_{M}=-\frac{1}{2} \bar{\nu}_{R}^{C} M_{R} \nu_{R}+\text { h.c. }
$$


where $M_{R}$ is a constant and $\nu_{R}^{C}$ is the charge conjugate of $\nu_{R}$. Adding this term to the Dirac mass term in the Langrangian, one obtains:

$$
-\mathcal{L}=m_{D} \bar{\nu}_{L} \nu_{R}+\frac{1}{2} \bar{\nu}_{R}^{C} M_{R} \nu_{R}+\text { h.c. }
$$

The combined mass matrix needs to be diagonalized leading to the "see-saw" mechanism. The eigenvalues of the matrix $M$

$$
M=\left[\begin{array}{cc}
0 & m_{D} \\
m_{D} & M_{R}
\end{array}\right]
$$

are

$$
\lambda_{ \pm}=\frac{M_{R} \pm \sqrt{M_{R}^{2}+4 m_{D}^{2}}}{2}
$$

The large value, $\lambda_{+} \approx M_{R}$, is associated with a sterile neutrino while the small one, $\lambda_{-} \approx$ $\frac{-m_{D}^{2}}{M_{R}}$, with the Standard Model light neutrinos.

\subsection{The Evidence for Neutrino Oscillation}

A few of the most important solar, reactor, atmospheric, and accelerator neutrino oscillation experiments are discussed below.

\subsubsection{Solar neutrino oscillations}

The main source of solar neutrinos are the three pp chains of nuclear reactions, which convert protons into ${ }^{4} \mathrm{He}$, alpha particle. These reactions take place in the solar core. In the $\mathrm{pp}$ chain there are five reactions that produce neutrinos and each of them produce a characteristic energy spectrum as shown in Figure 3.1. The majority of the neutrinos come from the initial reaction $p+p \rightarrow d+e+\nu_{e}$. However, they carry low energy and detecting them presents an experimental challenge. Most detectors makes use of boron- 8 neutrinos even though they are less abundant.

A pioneering solar neutrino experiment was started by Davis et al. [10] in the late 1960s. It took place in the Homestake mine in South Dakota using a method proposed by 


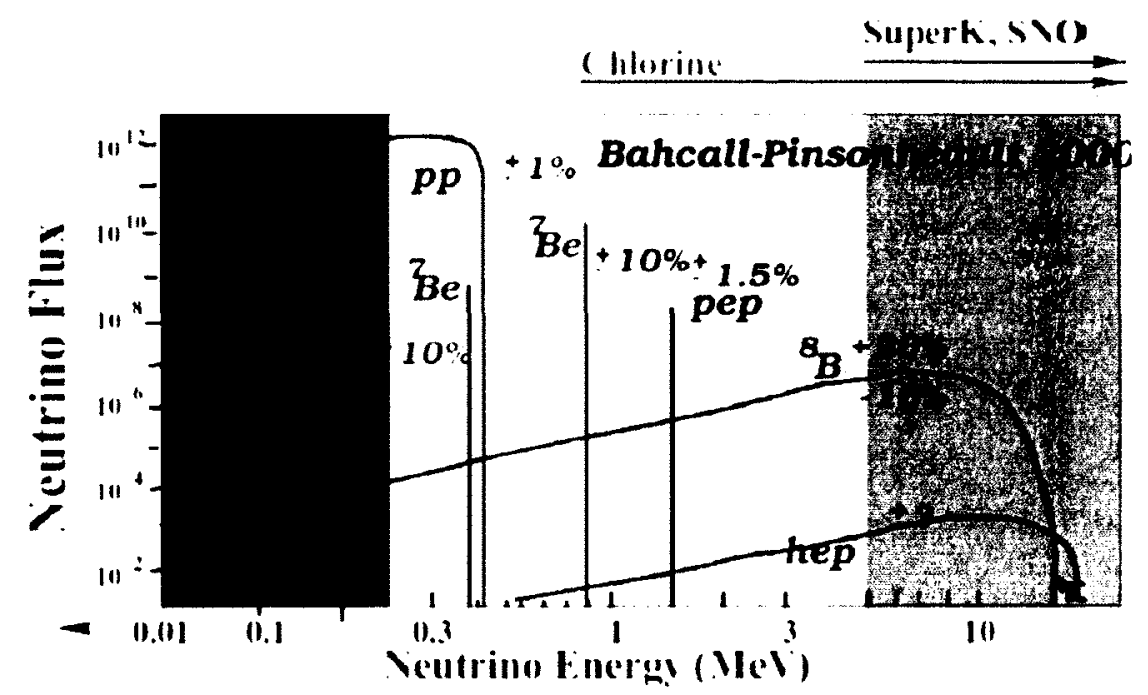

Figure 3.1: The energy spectra for various processes in the Sun. The energy range covered by different experimental techniques is also displayed.

Pontecorvo [11]. They exploited $\nu_{e}$ absorption on ${ }^{37} \mathrm{Cl}$ nuclei:

$$
\nu_{e}+{ }^{37} \mathrm{Cl} \rightarrow{ }^{37} \mathrm{Ar}+e^{-}
$$

where the ${ }^{37} \mathrm{Ar}$ atoms are radioactive. The reaction products were chemically extracted and introduced into a low-background counter. Only about $1 / 3$ of the total expected neutrino event rate was observed. This deficit has been called "the solar neutrino problem".

Gallium experiments (GALLEX, GNO and SAGE $[12,13,14,15,16])$ are sensitive to the most abundant pp solar neutrinos by utilizing the reaction:

$$
\nu_{e}+{ }^{71} \mathrm{Ga} \rightarrow{ }^{71} \mathrm{Ge}+e^{-}
$$

They too had found a deficit for CC electron neutrino interactions.

Besides, the chemical extraction experiments, mentioned above, there are also scattering experiments. The Kamiokande experiment in Japan was a water-Cherenkov detector that utilized $\nu_{e}$ scattering:

$$
\nu_{x}+e^{-} \rightarrow \nu_{x}+e^{-}
$$

This type of experiment can take advantage of the directional correlation between the incoming neutrino and the recoil electron. The Kamiokande result gave the first direct 
evidence that neutrinos come from the direction of the Sun [17]. Later the Kamiokande experiment was replaced with the high-statistics Super-Kamiokande with 50-kton water Cherenkov detector $[18,19,20,21]$. It also observed a deficit in water.

It should be noted that chlorine-based, gallium-based, and water-based experiments all measured different levels of deficit. Since each type of nucleus has a different low energy threshold for observation of CC events, one can conclude that the deficit is an energy dependent effect. Furthermore, those experiments rely on the $\mathrm{CC}$ interactions. Due to the very low energy of neutrinos from the Sun, the CC interaction could not occur for $\nu_{\mu}$ or $\nu_{\tau}$ even if they were produced through oscillations. The reason for this is the high mass of the $\mu(106 \mathrm{MeV})$ and $\tau(1.8 \mathrm{GeV})$. These experiments could only observe the disappearance of $\nu_{e} \mathrm{~s}$ but not reappearance in a form of other flavours. At that time, neutrino oscillation was still arguable.

The result that demonstrated that the solar neutrino problem was due to neutrino oscillation came from the Sudbury Neutrino Observatory (SNO) experiment [22]. SNO is also a Cerenkov experiment using 1 kton of heavy water as a target, which gives the experiment sensitivity to NC scattering by any flavour neutrino:

$$
\nu_{x}+d \rightarrow \nu_{x}+p+n
$$

In addition to:

$$
\nu_{x}+e^{-} \rightarrow \nu_{x}+e^{-}
$$

and

$$
\nu_{e}+d \rightarrow p+p+e^{-}
$$

In their first measurement, SNO saw a deficit consistent with the other measurements [23]. Their next result involved the NC interaction, which is flavour blind, and reported the total NC flux which agrees with the theoretical prediction [24]. Thus, confirming the oscillations of $\nu_{e}$ s to other flavours.

\subsubsection{Reactor neutrino oscillations}

The second experiment that indisputably demonstrated the effect of neutrino oscillation was KamLAND [25]. It is a liquid scintillator detector located in the Kamioka mine next to the Super-Kamiokande detector in Japan. It detects $\bar{\nu}_{e}$ from nuclear reactors via CC 
interaction, similarly to Cowan-Reines experiment described earlier :

$$
\bar{\nu}_{e}+p \rightarrow e^{+}+n
$$

The electron antineutrinos are produced with energies around $5 \mathrm{MeV}$. KamLAND utilizies the mean baseline distance of about $180 \mathrm{~km}$. It has a sensitive $\Delta m^{2}$ range down to about $10^{-5} \mathrm{eV}^{2}$. Since reactors produce pure beams of $\bar{\nu}_{e}$, KamLAND tries to measure a deficit in the number of anti-neutrinos observed, it is a so-called disappearance experiment. KamLAND observed for the first time the periodic nature of the survival probability expected from neutrino oscillations as shown in Figure 3.2. By combining KamLAND's and solar neutrino experimental results, one can obtain an allowed region in terms of the oscillation parameters as shown in Figure 3.3. The experiments using the solar neutrinos have large statistical power but poor energy resolution, which is illustrated by the vertical ellipse. On the other hand, KamLAND does not have good statistics but knows the energy.

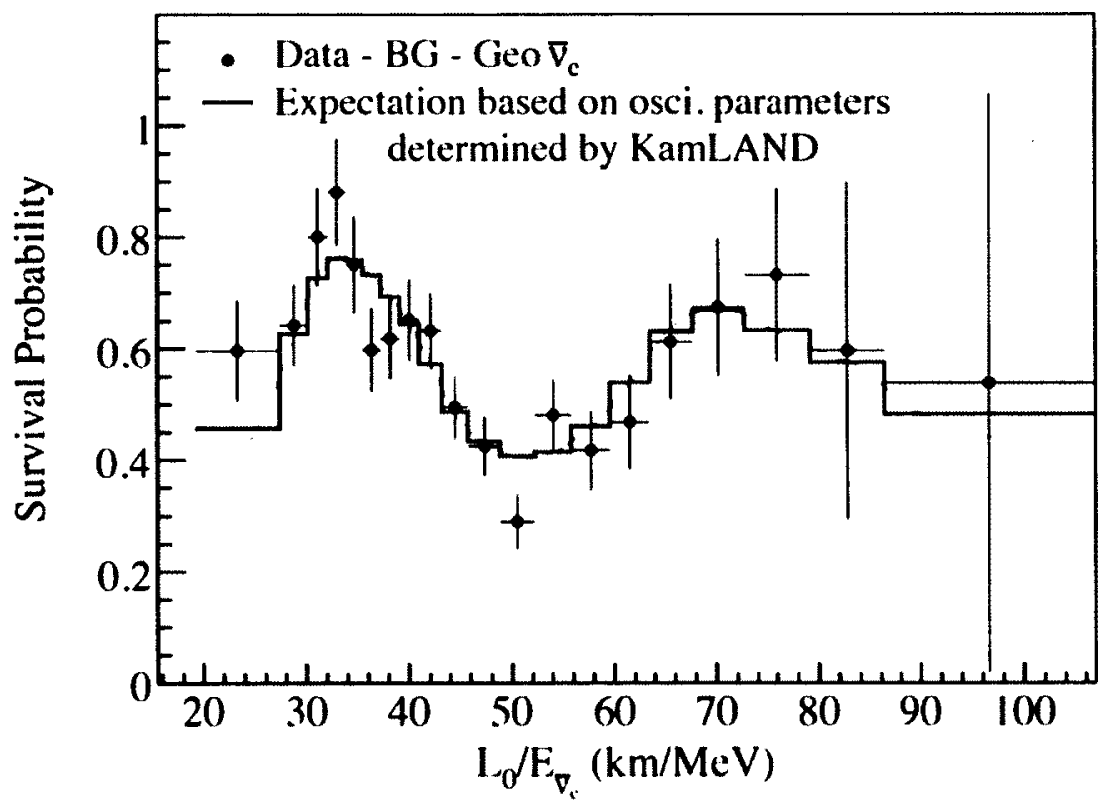

Figure 3.2: KamLAND's ratio of observed to expected anti-neutrino rates as a function of average L/E. Since neutrino sources are at different baselines, KamLAND assigns all the events to an average L.

\subsubsection{Atmospheric neutrino oscillations}

Atmospheric neutrinos come from the high energy cosmic rays collisions with the nuclei of the earth's atmosphere. Products of these interactions are $\pi$ and $\mathrm{K}$ mesons which then decay 


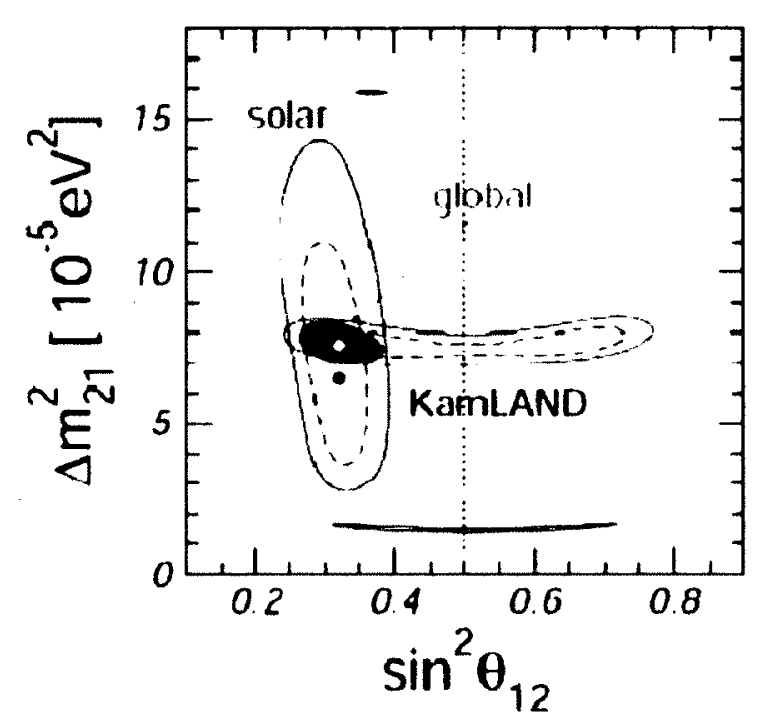

Figure 3.3: Allowed region for the solar oscillation parameters.

into mostly muon neutrinos and antineutrinos. Super-Kamiokande experiment was the first one that presented a compelling evidence for the neutrino oscillation using the atmospheric neutrinos. It consists of 50 kiloton water Cerenkov detector and utilizes photomultiplier tubes to catch the Cerenkov radiation. The electron coming from the electron-neutrino and muon coming from the muon-neutrino produce Cerenkov radiation by $\mathrm{CC}$ interactions in the water. They can be distinguished as they produce different signature, however, the lepton charge cannot be determined.

Super-Kamiokande can plot the flux of e-like and $\mu$-like events as a function of the zenith-angle as shown in Figure 3.4 [26]. In this plot, $\cos \theta=1$ corresponds to downwards direction of travel for neutrinos. On the other hand, $\cos \theta=-1$ corresponds to upward direction of travel. Events are divided into sub-GeV and multi-GeV events. It is seen that for $\mu$-like events the distribution deviates from the expectation but the distribution of e-like events is consistent with the expectation. This feature can be interpreted that muon neutrinos coming from below, meaning coming from the opposite side of the earth's atmosphere, oscillate into other neutrinos, while muon neutrinos coming from above the detector do not oscillate. Since there is no indication of appearance of electron neutrinos, it can be interpreted that muon neutrinos oscillated into tau neutrinos.

Since not everyone was convinced of the nature of the muon-neutrino disappearance, and there were speculations of exotic nature of neutrino, the characteristic behaviour of 
neutrino oscillation was examined. That is, the conversion probability as a function of $\mathrm{L} / \mathrm{E}$ (neutrino energy/baseline distance) was studied. A dip in the distribution was observed as shown in Figure 3.5, which cannot be explained by then thought exotic nature of a neutrino.
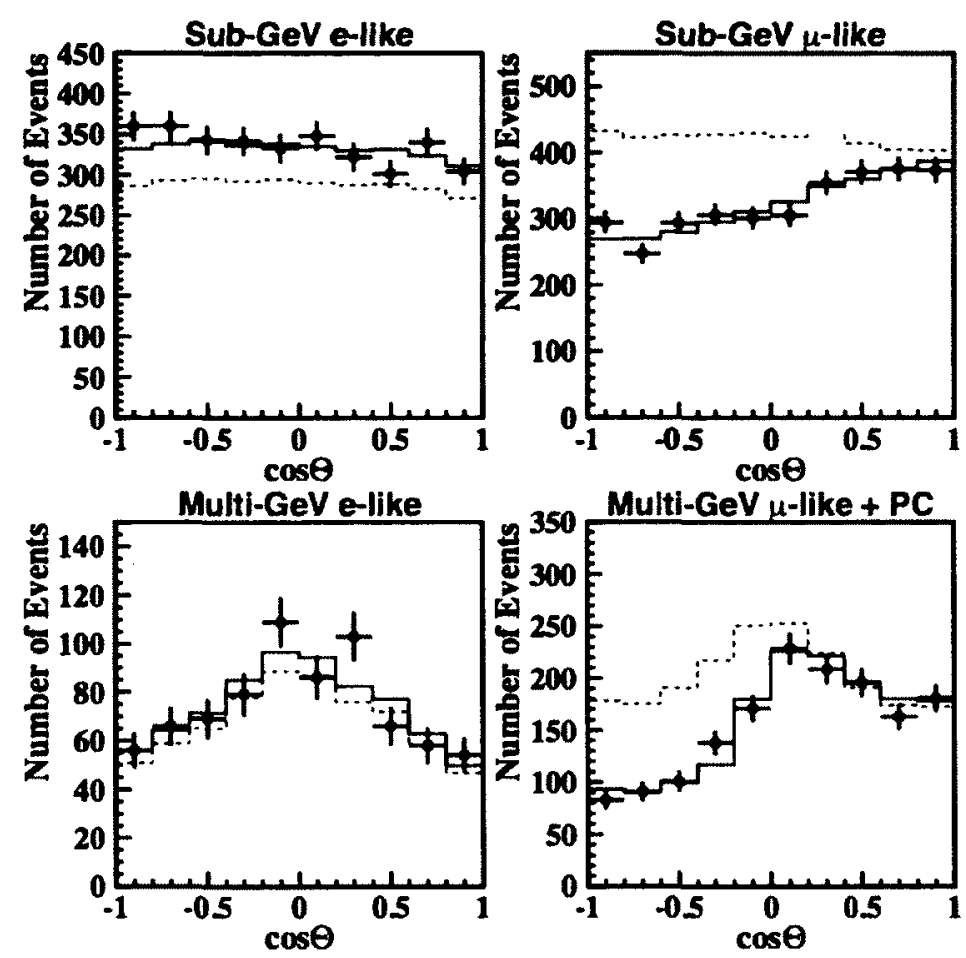

Figure 3.4: The zenith angle distribution for e-like and $\mu$-like events in sub-GeV and multi-GeV visible energy in Super-Kamiokande. The dotted histograms show the non-oscillated Monte Carlo events. The solid histograms show the best-fit expectations for $\nu_{\mu} \rightarrow \nu_{\tau}$ oscillations.

\subsubsection{Accelerator neutrino oscillations}

Production of accelerator neutrinos is similar to the one of atmospheric neutrinos in a sense that one takes advantage of a high energy proton collision producing mesons, which then decay to neutrinos. The so-called long-baseline experiments have a great sensitivity to oscillation parameters. They consist of a near detector, usually built near the beam production point, and a far detector with $\mathrm{L} \approx$ several hundred $\mathrm{km}$. The near detector measures the neutrino beam before oscillations take place, while the far detector makes the actual physics measurements. The two detectors should be built using the same technology in order to minimize systematic effects.

We distinguish two types of neutrino oscillation experiments: 


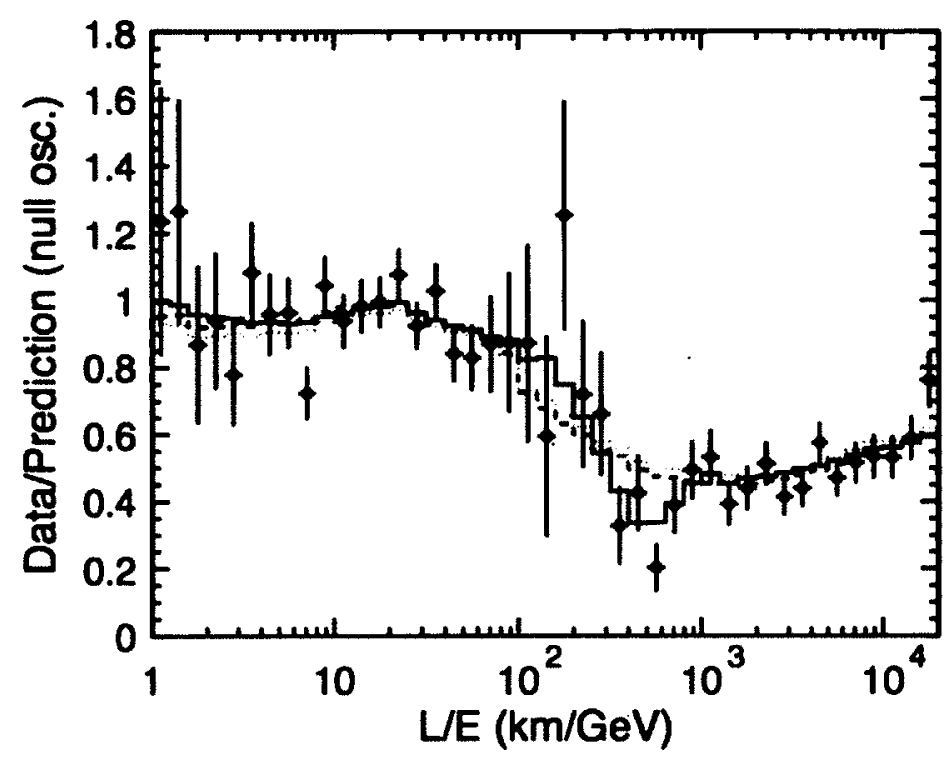

Figure 3.5: The ratio of the data to Monte Carlo prediction without oscillations as a function of $\mathrm{L} / \mathrm{E}$ in Super-Kamiokande. The solid histograms show the best-fit expectations for $\nu_{\mu} \rightarrow \nu_{\tau}$ oscillations.

1. Disappearance Experiment: one observes the energy spectrum of a neutrino beam at the beam source, and at the far detector where oscillations should have occurred. The ratio of these two spectra should indicate an oscillatory pattern. An example is shown in Figure 3.6. The dip indicates that some neutrinos oscillated and its depth is used to measure the mixing angle $\sin ^{2}(2 \theta)$. The position of the dip on the energy axis serves to extract $\Delta m^{2}$. The key of this type of experiment is to be able to precisely understand the beam before oscillations take place.

2. Appearance Experiment: this type of experiment searches for the appearance of a neutrino flavour in a beam that initially was not present. The biggest challenge of this experiment is understanding all the background that could mimic the appearance signature.

The K2K (KEK-to-Kamioka) long-baseline neutrino oscillation experiment is the first accelerator-based experiment [27]. It is a disappearance experiment aiming at neutrino oscillation in muon neutrinos. The spectrum of the neutrino beam was measured by a near detector located $300 \mathrm{~m}$ from the production target. The average neutrino energy was $\sim 1.3$ $\mathrm{GeV}$ and $\mathrm{L}=250 \mathrm{~km}$. The measured energy spectrum showed the distortion expected from neutrino oscillations. 


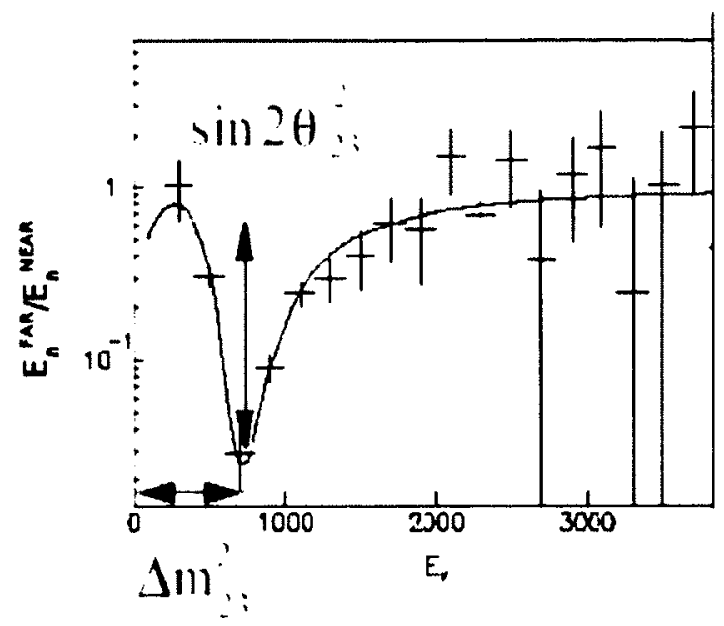

Figure 3.6: The ratio of energy spectrum in the far detector to the one in the near detector (after oscillation/before oscillation).

MINOS is the second long-baseline experiment with the baseline distance of $735 \mathrm{~km}$. The far detector's total mass is 5.4 kton and is iron-scintillator tracking calorimeter with toroidal magnetic field, based in Soudan, Northern Minnesota. Similarly, the near detector is also iron-scintillator tracking calorimeter with toroidal magnetic field with a total mass of $0.98 \mathrm{kton}$, based at Fermilab. It is positioned about $1 \mathrm{~km}$ away from the beam source and monitors the beam flux and initial composition. Since the detector is magnetized, the charged current interactions of $\nu_{\mu}$ can be distinguished from $\bar{\nu}_{\mu}$. Its latest results are shown in Figure 3.7 [28]-[31]. The figure on the left shows the neutrino energy spectrum as measured by the MINOS far detector. One clearly sees the deficit towards lower energies. The figure on the right shows the data divided by the expected flux, where one sees a characteristic dip behaviour.

Even though both atmospheric and accelerator long-baseline oscillations seem to be consistent with the muon-neutrino to tau-neutrino oscillations, detecting appearance of tau is challenging. OPERA is an experiment whose main goal is to detect $\tau$ neutrinos in an initially muon-neutrino beam for the first time. Thus, it is attempting to directly observe the oscillations of muon-neutrinos to tau-neutrinos instead of just measuring the disappearance of muon-neutrinos. It is an accelerator long-baseline experiment utilizing emulsion technique to identify short-lived $\tau$ leptons. It has a baseline of $730 \mathrm{~km}$ with a neutrino source at CERN and a detector at Gran Sasso, Italy. The average neutrino energy is $\sim 17 \mathrm{GeV}$. OPERA uses bricks, composed of photographic emulsion film, as its detection 

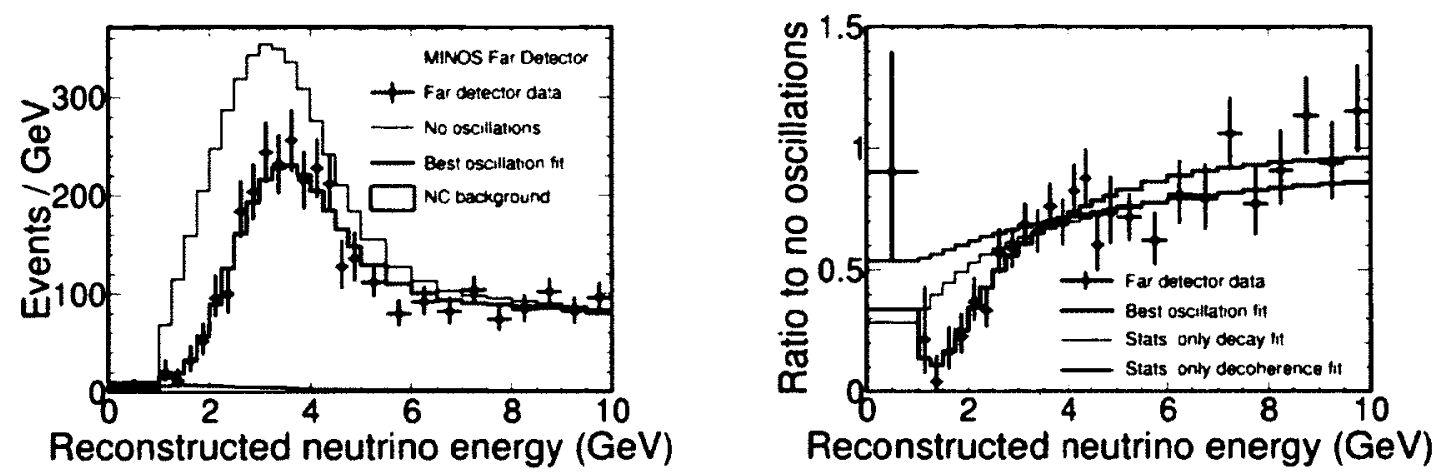

Figure 3.7: The energy spectrum of MINOS charged current events in the far detector. The red line represents the spectrum in the absence of oscillations. The blue line represents a fit to a 2 flavour oscillation model. The plot of on the right shows the fraction of data to expected data without oscillations. The plot has a characteristic oscillation behaviour. It is seen that the neutrino oscillation hypothesis best fits the data.

medium. The film provides sub-mm resolution that is used to identify the short-lived tau decay kink. So far OPERA have reported observation of one $\nu_{\tau}$ candidate $[32,33]$.

\subsection{Summary and Outstanding Questions}

Based on the atmospheric and solar oscillation experiments, there are two squared mass differences called $\Delta m_{\text {solar }}^{2}$ and $\Delta m_{\text {atmos }}^{2}$. The highest $\Delta m^{2}$ was first observed using neutrinos produced in the upper atmosphere and the lower one was observed with neutrinos from the Sun. The smaller $\Delta m^{2}$ is identified with the mass splitting between $\nu_{1}$

and $\nu_{2}: \Delta m_{12}^{2}=\Delta m_{\text {solar }}^{2}$. The atmospheric deficit combines $\Delta m_{23}^{2}$ and $\Delta m_{13}^{2}$. Since $\Delta m_{13}^{2}=\Delta m_{23}^{2}+\Delta m_{12}^{2}$ and $\Delta m_{12}^{2}$ is small, $\Delta m_{13}^{2} \approx \Delta m_{23}^{2} \approx \Delta m_{\text {atmos }}^{2}$. Currently, experiments paint a consistent three neutrino oscillations picture with five free parameters. The neutrino mass states are different from the flavour states. The two mass splittings are:

$$
\begin{aligned}
& \Delta m_{12}^{2}=-7.59_{-0.21}^{+0.20} \times 10^{-5} \mathrm{eV}^{2} \\
& \left|\Delta m_{23}^{2}\right|=2.43_{-0.10}^{0.06} \times 10^{-3} \mathrm{eV}^{2}
\end{aligned}
$$

Current experiments have not determined whether the solar pair is at the bottom or top of the spectrum. These two possibilities are referred to as a normal and inverted spectrum, respectively, as shown in Figure 3.8. 


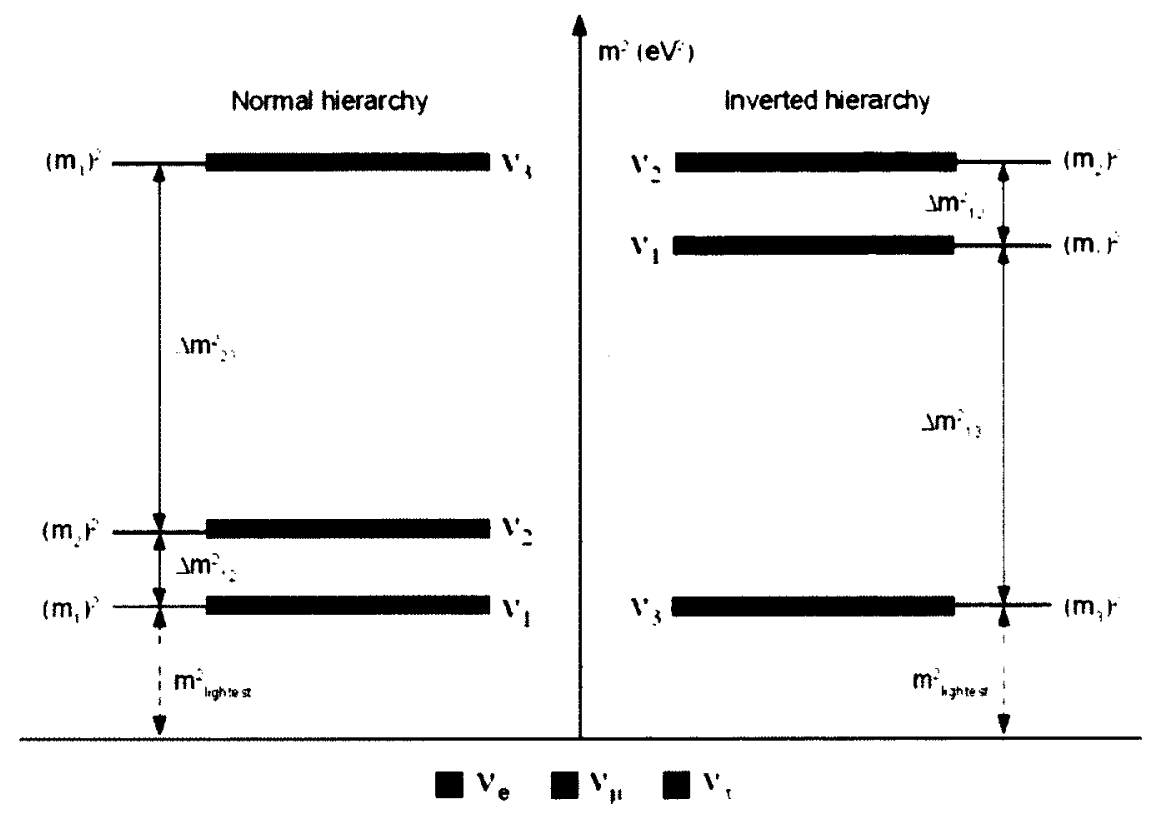

Figure 3.8: A three-neutrino squared-mass spectrum accounting for the observed solar, reactor, atmospheric and accelerator neutrinos. The spectrum on the left is referred to as "normal" and on the right as "inverted" hierarchy. Oscillation experiments cannot determine how far above zero the entire spectrum lies.

The mixing angles are measured to be:

$$
\begin{gathered}
\theta_{12}=34.06_{-0.84}^{+1.16 \circ} \\
\sin ^{2} \theta_{23}=0.386_{0.014}^{+0.024} \\
\theta_{13}=9.0_{-0.5}^{+0.4 \circ}
\end{gathered}
$$

Several important questions about neutrino properties still remain unanswered. Below, there is a list followed by a brief discussion of few of them.

- Are there more than 3 neutrinos?

A puzzling result was obtained by LSND collaboration [34]. LSND was a tank filled with mineral oil doped with a scintillating compound. It used a beam of $800 \mathrm{MeV}$ protons to produce mainly $\bar{\nu}_{\mu}$. The detector was placed $30 \mathrm{~m}$ from the source of neutrinos and searched for appearance of $\bar{\nu}_{e}$ above the background. The experiment 
looked for $\bar{\nu}_{e}$ interactions using inverse beta decay $\bar{\nu}_{e}+p \rightarrow e^{+}+n$. The final state neutron was captured by the mineral oil to produce a $2.2 \mathrm{MeV}$ photon. The coincidence between the prompt $e^{+}$signal and delayed photon signal was used to distinguish the signal events from the background. LSND reported excess of $\bar{\nu}_{e}$ above that expected in the absence of neutrino oscillations. However, the distance of $30 \mathrm{~m}$ in LSND is too short for the muon neutrino to oscillate unless there exists at least one more flavour of neutrino. The best fit oscillation point is at $\left(\sin ^{2}(2 \theta), \Delta m_{23}^{2}\right)=$ $\left(0.003,1.2 \mathrm{eV}^{2}\right)$. The LSND value of $\Delta m^{2}$ is very different from the expected. It can be interpreted that beyond the $\Delta m_{\text {solar }}^{2}$ and $\Delta m_{\text {atmos }}^{2}$, there is also a third mass splitting. This in turn, implies that there are at least 4 neutrinos. However, as noted earlier the LEP result showed that there are only 3 flavours $[35,36]$. If there are more than three mass eigenstates, then there are linear combinations of them that do not couple to the $\mathrm{W}$ or $\mathrm{Z}$ bosons. These linear combinations are called sterile neutrinos and only interact via gravity.

The miniBooNE experiment investigated the LSND result $[37,38]$. The detector operated in the Fermilab Booster $\nu_{\mu}$ beam and looked for $\nu_{e}$ appearance. The beam consisted of $99.7 \%$ pure $\nu_{\mu} \mathrm{s}$ with energies approximately $1 \mathrm{GeV}$. The detector is a spherical tank containing pure mineral oil with diameter of $12 \mathrm{~m}$. MiniBooNE identifies particles based on the characteristic Cherenkov ring pattern that they create. The MiniBooNE was able to observe the same mass difference as the LSND. It looked for $\nu_{e}$ appearance by studying the charged current $\nu_{e}$ interactions in the oil. It found no excess of events in the region predicted by LSND. However, it observed a lower energy excess. MiniBooNE also just recently finished running in the anti-neutrino mode, as LSND, and as with neutrino mode it found an excess at lower energies. Thus, in addition to the excess from LSND, we now also have an excess from the MiniBooNE data.

- Is the hierarchy normal or inverted?

In order to determine this, one can study the matter effect on $\nu_{\mu} \rightarrow \nu_{e}$ and $\bar{\nu}_{\mu} \rightarrow \bar{\nu}_{e}$. One needs to pick a correct $\mathrm{L} / \mathrm{E}$ to be sensitive to the atmospheric mass gap, $\Delta m_{32}^{2}$. As mentioned earlier, this quantity is positive for a normal spectrum, and negative for the inverted hierarchy. Since the study involves $\nu_{e}$ and $\bar{\nu}_{e}$, the interaction leads to extra potential energy $V_{W}= \pm \sqrt{2} G_{F} N_{e}$, where the quantity is positive for neutrinos 
and negative for antineutrinos. The sign of $\Delta m_{32}^{2}$ can be determined since we know the sign of the extra energy from the Standard Model.

- What are the masses of the neutrino mass eigenstates?

The neutrino oscillation experiments are not sensitive to the individual neutrino masses but only their mass differences. Thus, they cannot perform a direct measurement. Direct information about their masses comes from, for example, tritium $\beta$ decay.

- Is $\mathrm{CP}$ invariance violated in the neutrino sector?

This is an important question as it may help explain the imbalance of matter and antimatter in the universe. It is believed that the Big Bang produced equal amounts of matter and antimatter. There must have been some asymmetry present at the beginning that required the matter to behave differently from antimatter. This asymmetry could have been the violation of CP invariance. Since it is known that the CP violation in the quark sector is not adequate to explain the observed imbalance, it is important to check if leptonic CP violation could explain it. A natural way for the matter-antimatter asymmetry to arise is through the see-saw mechanism described earlier. The process associates each light neutrino $\nu$ with a heavy partner $\mathrm{N}$. The masses of the heavy neutrinos are believed to be in the range $10^{9-15} \mathrm{GeV}$ and would have been produced in the Big Bang. According to the see-saw model both the light and the heavy neutrinos are Majorana particles. This would lead to the CP violation in the leptonic decay of $\mathrm{N}$. In the laboratory, the CP invariance could be demonstrated on light neutrinos by showing that the probability of oscillation $\nu_{\alpha} \rightarrow \nu_{\beta}$ is different from $\bar{\nu}_{\alpha} \rightarrow \bar{\nu}_{\beta}$.

- Are neutrinos their own anti-particles?

A neutrino that is identical to its antiparticle is referred to as a Majorana particle, while the one that is not its own antiparticle is referred to as a Dirac particle. As noted previously, the total lepton number $\mathrm{L}$ is conserved for Dirac but not for Majorana particles. Confirmation that $\mathrm{L}$ is not conserved could come from observation of neutrinoless double beta decay $(0 \nu \beta \beta), A(Z) \rightarrow A(Z+2)+2 e^{-}$, in which one nucleus decays to another with the emission of two electrons. The process would violate total lepton number by $\Delta L=2$ and can only occur if neutrino is its own antiparticle. 
In order to address these questions a precision neutrino detector is needed. ArgoNeuT experiment represents a crucial step towards realization of a kiloton-scale precision Liquid Argon Time Projection Chamber (LArTPC) detector. ArgoNeuT has fulfilled its R\&D goals. Furthermore, it has been demonstrating its super technological power through various physics measurements, in particular the one presented in this thesis. An ultra-large detector build on the line pioneered by ArgoNeuT will be able to address the mass hierarchy and $\mathrm{CP}$ violation in addition to many more important topics. 


\section{$4 \quad$ Neutrino Scattering}

Neutrino interactions present unique opportunities for exploration of fundamental questions in physics. In hadronic and nuclear physics they can address electroweak form factors, the strange quark content of the nucleon and neutrino-induced pion production. Since neutrinos have no charge their presence can be inferred by studying secondary particles they create after colliding and interacting with matter. Thus, the study of cross sections is needed for studies of neutrino interactions and neutrino oscillations. In this section, a brief overview of cross section is given followed by the cross section for Charged Current Quasi Elastic (CCQE) interaction. This interaction refers to single nucleon knockout without pion production and constitutes the biggest fraction of $\mu+N p$ topological analysis, according to the $\mathrm{MC}$, presented in this thesis. For $\mu+N p$ process the description of the cross section would rely on multi-channel calculation, where all processes that produce that final state would have to be included.

\subsection{Cross Section}

For particles 1 and 2 colliding and producing particles $3,4, \ldots, \mathrm{n}$ the scattering cross section is given by:

$$
\begin{array}{r}
\sigma=\frac{S \hbar^{2}}{4 \sqrt{\left(p_{1} \cdot p_{2}\right)^{2}-\left(m_{1} m_{2} c^{2}\right)^{2}}} \int|M|^{2}(2 \pi)^{4} \delta^{4}\left(p_{1}+p_{2}-p_{3} \ldots-p_{n}\right) \\
\times \prod_{j=3}^{n} \frac{1}{2 \sqrt{p_{j}^{2}+m_{j}^{2} c^{2}}} \frac{d^{3} p_{j}}{(2 \pi)^{3}}
\end{array}
$$

where $\mathrm{S}$ is a statistical factor that corrects for double-counting when there are identical particles in the final state. $M$ is the amplitude, which contains the dynamics of the process 
and is a function of various momenta. It is calculated by evaluating appropriate Feynman diagrams.

For point-like particles this amplitude can be calculated analytically. As an example, one can consider inverse muon decay process, for which the cross section derivation is given by [39]

$$
\nu_{\mu}+e^{-} \rightarrow \mu^{-}+\nu_{e}
$$

The lowest order diagram representing this process is shown in Figure 4.1

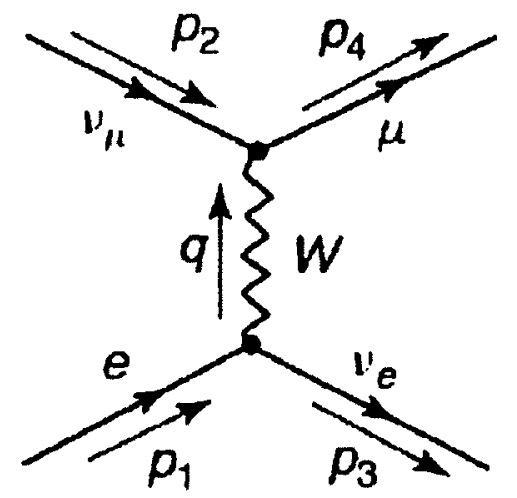

Figure 4.1: Lowest order Feynman diagram for inverse muon decay

After assuming $q^{2} \ll M_{w}^{2} c^{2}$ and using the simplified propagator the amplitude is:

$$
M=\frac{g_{w}^{2}}{8}\left[\bar{u}(3) \gamma^{\mu}\left(1-\gamma^{5}\right) u(1)\right] \frac{i g_{\mu \nu}}{(M c)^{2}}\left[\bar{u}(4) \gamma_{\mu}\left(1-\gamma^{5}\right) u(2)\right]
$$

Making use of trace theorems, it follows that:

$$
\sum_{\text {spins }}|M|^{2}=4\left(\frac{g_{w}}{M_{w} c}\right)^{4}\left(p_{1} \cdot p_{2}\right)\left(p_{3} \cdot p_{4}\right)
$$

Summing over all final spins, two for electron and one for neutrino, and averaging over initial spins:

$$
<|M|^{2}>=2\left(\frac{g_{w}}{M_{w} c}\right)^{4}\left(p_{1} \cdot p_{2}\right)\left(p_{3} \cdot p_{4}\right)
$$

Going to the CM frame and neglecting the mass of the electron:

$$
<|M|^{2}>=8\left(\frac{g_{w}}{M_{w} c}\right)^{4}\left[1-\left(\frac{m_{\mu} c^{2}}{2 E}\right)^{2}\right]
$$


where $\mathrm{E}$ is the initial neutrino energy. Making use of the differential scattering cross section for two-body scattering:

$$
\frac{d \sigma}{d \Omega}=\left(\frac{\hbar c}{8 \pi}\right)^{2} \frac{S|M|^{2}}{\left(E_{1}+E_{2}\right)^{2}} \frac{\left|p_{f}\right|}{\left|p_{i}\right|}
$$

where $\left|p_{f}\right|$ is the magnitude of the outgoing momentum and $\left|p_{i}\right|$ is the magnitude of the incoming momentum, the differential scattering cross section for inverse muon decay can be written as:

$$
\frac{d \sigma}{d \Omega}=\frac{1}{2}\left[\frac{\hbar c g_{w}^{2} E}{4 \pi\left(M_{w} c^{2}\right)^{2}}\right]^{2}\left[1-\left(\frac{m_{\mu} c^{2}}{2 E}\right)^{2}\right]^{2}
$$

The neutrino scattering of a nucleus is a far more complicated process due to a structure of a nucleon and the presence of gluons, among other things. Thus, it is not possible to analytically calculate the matrix amplitude $\mathrm{M}$, as was presented above. An example of a method for Charged Current Quasi-Elastic cross section will be addressed in the following section.

The neutrino-nucleus interactions can be separated into three distinct energy areas classified as low, medium and high. The wave-length scale of the interaction in the 'low' energy is greater than the nuclear diameter and so initial and final states are specific nuclear levels. In the medium energy region the interaction length is hadronic (about $1 \mathrm{fm}$ ) and nuclear effects play a big role. In the high energy regime, the scale becomes partonic (about $0.1 \mathrm{fm}$ ) and nuclear effects are not so significant. The cross sections relevant for ArgoNeuT are at the medium energy region referring to neutrino energies of about $0.1-20 \mathrm{GeV}$. The scattering in this regime is complicated as variety of mechanisms are crucial. The main three processes are:

- Quasi-Elastic Scattering: takes place when a neutrino elastically scatters off an entire nucleon and liberates it in the process

- Resonance Production: takes place when a neutrino excites a target nucleon to a resonance state. The resonant state can then decay to different final states producing mesons and nucleons.

- Deep Inelastic Scatterring: takes place when an energetic neutrino interacts on a quark level. The final state presents itself in a form of a hadronic shower. 
As can be already deducted from the description of these interaction, they include final states with nucleons, pions, kaons and mesons. Furthermore, there are nuclear effects that can impact all of these processes and change what is emitted in the final state making the description challenging from the theory point of view. It should also be pointed out that there exist theoretical descriptions of these scattering processes but there is no uniform theory which addresses the transition between them or how they should be combined. What has been measured to this day together with a NUANCE Monte Carlo [40] prediction for neutrinos and anti-neutrinos is shown in Figure 4.2. One can immediately notice that there have been many more experiments performed using neutrinos as opposed to antineutrinos, which is attributed to low statistics. Furthermore, one sees that most cross section measurements were done for higher energies and there is a clear lack of any data in lower regime, evident especially for anti-neutrinos. Thirdly, one notices a linear energy dependance of cross section. This behaviour is attributed to Deep Inelastic Scattering, which presents itself with scattering off quarks as discussed earlier.

\subsection{Charged Current Quasi-Elastic Scattering}

The Charged Current Quasi-Elastic interaction for neutrinos involves conversion of neutron into proton. For anti-neutrinos, on the other hand, proton is converted into neutron:

$$
\begin{gathered}
\nu_{\mu}+n \rightarrow \mu^{-}+p \\
\bar{\nu}_{\mu}+p \rightarrow \mu^{+}+n
\end{gathered}
$$

These interactions were studied in the past, starting from 1970's, by deuterium-filled bubble chambers. Their cross section was calculated by relying on Llewellyn-Smith approach from 1972 [102]. The Quasi-Elastic (QE) cross section off free nucleus is expressed as:

$$
\frac{d \sigma}{d Q^{2}}=\frac{G_{F}^{2} M^{2}\left|V_{u d}\right|^{2}}{8 \pi E_{\nu}^{2}}\left[A \pm \frac{(s-u)}{M^{2}} B+\frac{(s-u)^{2}}{M^{4}} C\right]
$$

where + refers to neutrinos and - for anti-neutrinos. $G_{F}$ is the Fermi coupling constant, $\mathrm{Q}$ is four-momentum transfer, $\mathrm{M}$ is the nucleon mass, $\mathrm{m}$ is lepton mass. Incident neutrino 

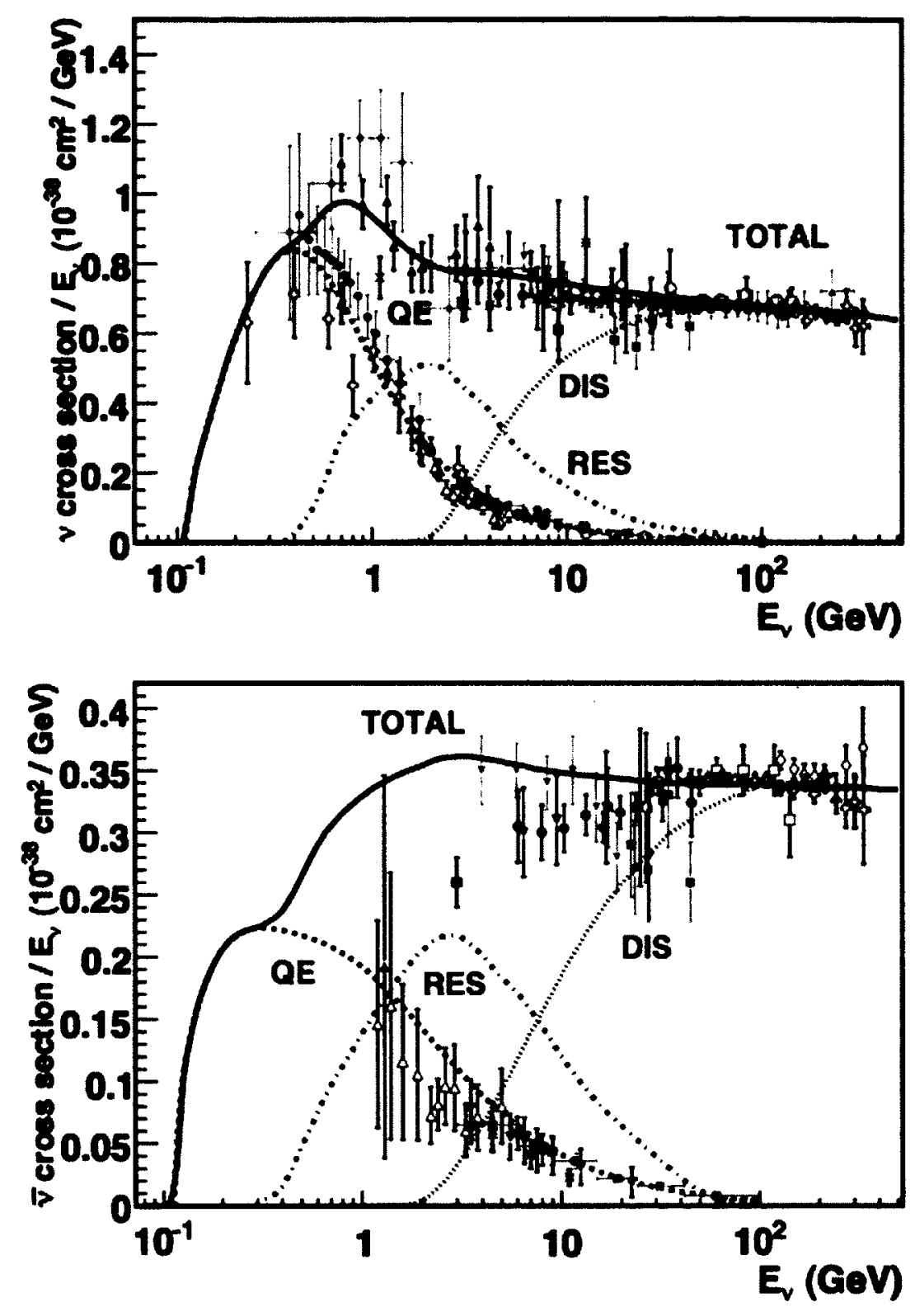

Figure 4.2: Total cross section per nucleon divided by neutrino energy and plotted as a function of energy for neutrinos (top) and anti-neutrinos (bottom). Contributions from Quasi-Elastic, Resonant and Deep Inelastic Scattering processes are shown as modeled by NUANCE generator [40]. The figure is adopted from $[60]$

energy is $E_{\nu}$, and $(s-u)=4 M E_{\nu}-Q^{2}-m^{2}$.

$$
\begin{array}{r}
A=\frac{m^{2}+Q^{2}}{M^{2}}\left[(1+\eta) F_{A}^{2}-(1-\eta) F_{1}^{2}+\eta(1-\eta) F_{2}^{2}+4 \eta F_{1} F_{2}\right. \\
\left.-\frac{m^{2}}{4 M^{2}}\left(\left(F_{1}+F_{2}\right)^{2}+\left(F_{A}+2 F_{P}\right)^{2}-\left(\frac{Q^{2}}{M^{2}}+4\right) F_{P}^{2}\right)\right]
\end{array}
$$




$$
\begin{gathered}
B=\frac{Q^{2}}{M^{2}} F_{A}\left(F_{1}+F_{2}\right) \\
C=\frac{1}{4}\left(F_{A}^{2}+F_{1}^{2}+\eta F_{2}^{2}\right)
\end{gathered}
$$

where $\eta=Q^{2} / 4 M^{2}$, and $F_{1}, F_{2}$ are vector form factors, $F_{A}$ is axial vector and $F_{P}$ pseudoscalar form factors of the nucleon.

This formalism was used for QE scattering data on deuterium with pseudoscalar contribution neglected. The vector form factors could be characterized from electron scattering. Thus, only the axial-vector form factor of the nucleon needed to be measured by neutrino experiments. Typically, this form factor assumes a dipole form:

$$
F_{A}\left(Q^{2}\right)=\frac{g_{A}}{\left(1+\frac{Q^{2}}{M_{A}^{2}}\right)^{2}}
$$

and depends on two empirical parameters: "axial mass", $M_{A}$, and axial vector form factor at $Q^{2}=0, g_{A}=F_{A}(0)=1.2694 \pm 0.0028$ [49]. The $g_{A}$ is determined from nuclear beta decay and so finding the axial mass became the focus of the neutrino community. By using mainly experimental data on deuterium the $M_{A}$ was calculated to be $1.026 \pm 0.021$ $\mathrm{GeV}[42]$.

Current neutrino experiments use far more complicated nuclei than deuterium and thus nuclear effects can cause modifications to the cross section presented in Equation 4.11. Since QE events compose the largest signal in the neutrino oscillation experiments, the new focus was modeling of QE scattering on nuclear targets.

The nucleus is usually described by using Impulse Approximation [43] where quasi-free nucleons participate in the scattering. Fermi Gas Model [44] is used for simulation of QE events although many sophisticated treatments were developed in recent years $[45,46,47$, $48,49,50,51,52,53]$. The recent experiments began repeating earlier measurements of QE cross sections and $M_{A}$. The axial mass values range from 1.05 to $1.35 \mathrm{GeV}[54,55,56,57$, $58,59]$ and most of them were found to be much larger than what was reported by earlier deuterium-based experiments. The higher $M_{A}$ implies higher cross section and Figures 4.3 and 4.4 summarize the current situation. The discrepancy between experiments is clearly visible with tension between MiniBooNE cross section [54] and NOMAD result [59] being in disagreement on the order of $30 \%$ despite being both on carbon target. 


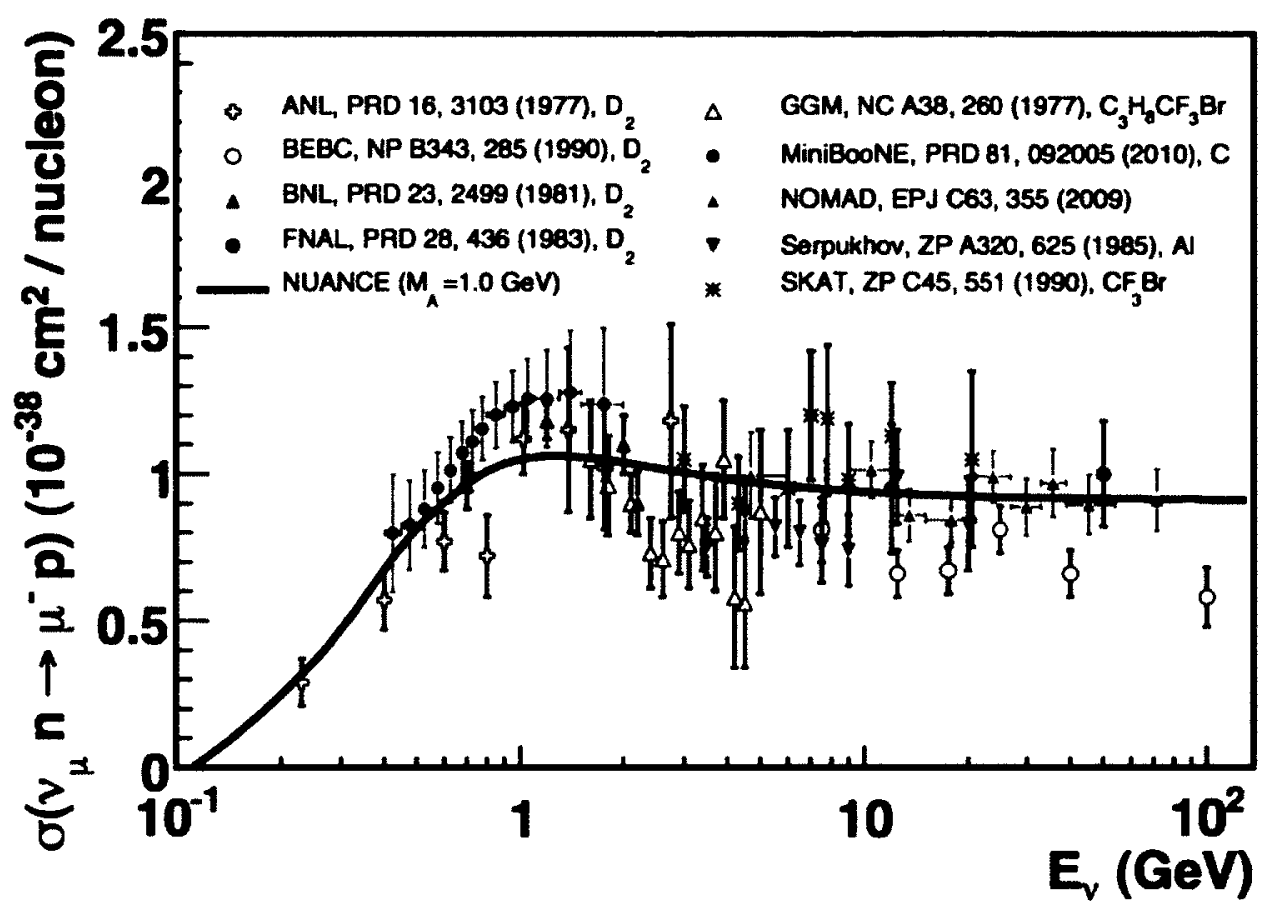

Figure 4.3: Quasi-Elastic scattering cross section as a function of neutrino energy for neutrinos. A variety of different measurements together with free nucleon scattering prediction with $M_{A}=1.0$ $\mathrm{GeV}$ are shown. The figure is adopted directly from $[60]$.

It is evident that the recent measurements raised many questions. It is believed that there need to be further improvements done to the Impulse Approximation in terms of nuclear effects. In order to better account for QE scattering nucleon-nucleon correlations and two-body exchange currents should be considered. One needs to be also careful when defining "QE" interaction. It will be shown in this thesis, that ArgoNeuT sees multiple nucleons in the final state and not just a single nucleon emission. It is clear that measurements of nucleon emission are needed to better understand nuclear effects and finals state interactions.

\subsection{Nuclear Effects}

\subsubsection{Final State Interactions}

Experiments run their selection methods based on what remains in the final state, subject to their detection efficiency. Final state interactions (FSI) refer to re-scattering of hadrons that were produced in the initial state as they are on their way to exit the nucleus. FSI can 


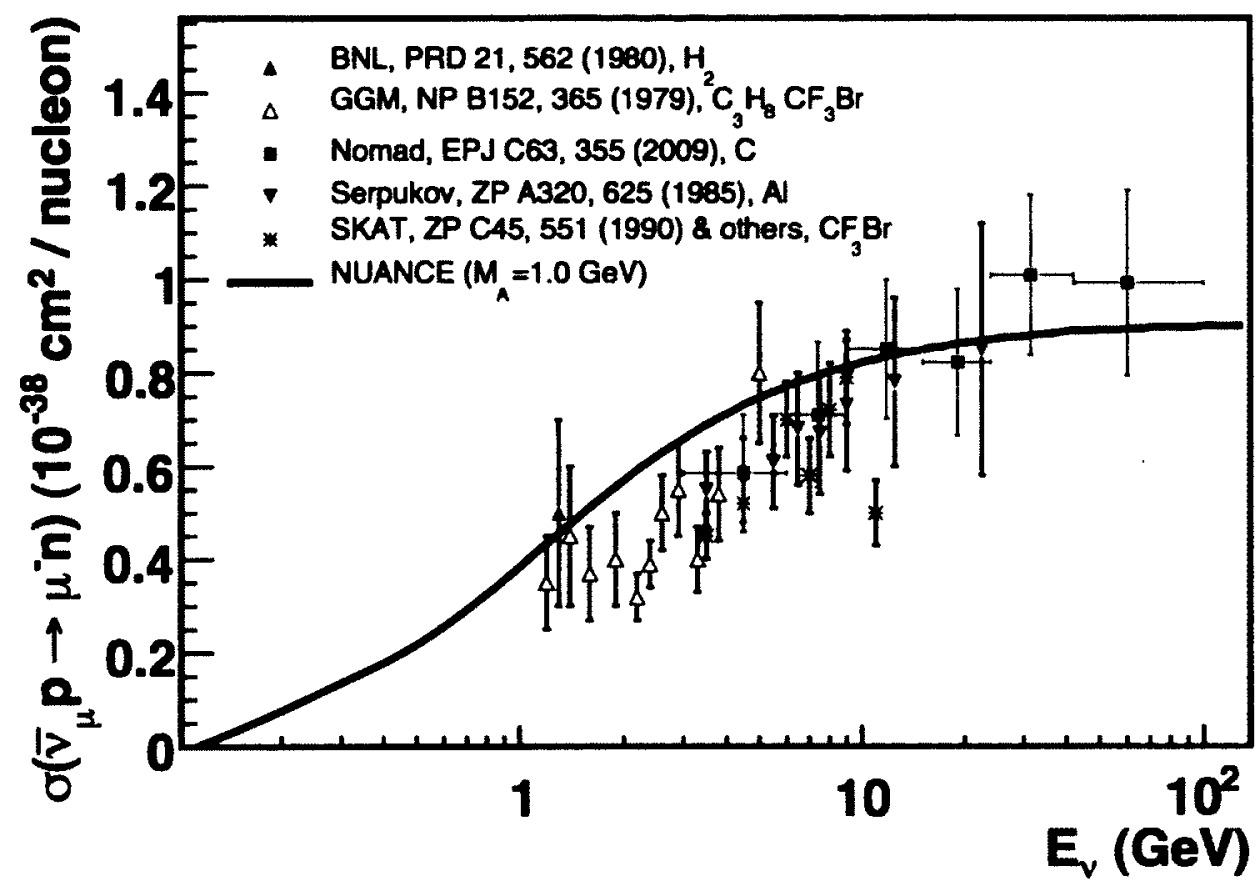

Figure 4.4: Quasi-Elastic scattering cross section as a function of neutrino energy for anti-neutrinos. A variety of different measurements together with free nucleon scattering prediction with $M_{A}=1.0$ $\mathrm{GeV}$ are shown. The figure is adopted directly from [60].

drastically alter what was initially produced by a neutrino. In case of CCQE interaction instead of one nucleon produced, FSI can lead to multi-nucleon knock-out and thus an increase in the number of emitted nucleons in the final state. Furthermore, pions and gammas can also be produced. Thus, FSI can lead to rejection of the CCQE interaction in the final event sample. On the other hand, an interaction which is not a CCQE process can change due to the FSI and mimic its signature leading to its acceptance in the final event sample. This is especially common in case of pion absorption in the nucleus. Modern experiments use heavy nuclear targets and thus this process cannot be ignored.

\subsubsection{Meson Exchange Currents}

FSI are not the only processes that takes place inside a nucleus. Electron scattering points out a more complicated mechanism in which an incoming electron interacts with two nucleons at the same time. In a correlated nucleon pair the partner nucleon is also ejected leading to the excitation of 2 particle-2 hole states $(2 \mathrm{p}-2 \mathrm{~h})$. However, $3 \mathrm{p}-3 \mathrm{~h}$ excitations 
are also possible [61]. In inclusive inelastic electron scattering on nuclei the $2 \mathrm{p}-2 \mathrm{~h}$ process becomes important at larger energy transfers beyond the Quasi-Elastic peak [62]. This two body interaction effect can also play a big role in neutrino experiments. It was pointed out that the $2 \mathrm{p}-2 \mathrm{~h}$ process could also contribute to the measured quasi-elastic-like cross section in MiniBooNE since the experiment is not sensitive to any outgoing nucleons [63]. Many models for neutrino-nucleus scattering try to implement this multi-nucleon emission effect but the precise strength and limits of its applicability are still not known. Th models also consider the virtual meson exchange between the two nucleons and collectively the mechanism is called Meson Exchange Current (MEC). 


\section{PART II}

\section{Neutrino Detection with the Liquid Argon Time Projection \\ Chamber Technique}




\section{The LArTPC concept}

The Liquid Argon Time Projection Chamber (LArTPC) concept was proposed in late seventies and has a long history of technological development in Europe [64, 65]. This work cumulated in a first detector, ICARUS T600, for underground physics application [66]. In the USA, the ArgoNeuT detector is the first to be exposed to a neutrino beam and in this role, a first step in the phased program towards the construction of a massive LArTPC detector for LBNE [67].

ArgoNeuT is a NSF/DOE research and development project at Fermilab and first ever LArTPC operated in a "low-energy" neutrino beam, referring to neutrino energies in the 0.5-10.0 GeV. These energies are significant for long-baseline neutrino oscillation searches as the oscillation probability is maximal in the few $\mathrm{GeV}$ region, assuming typical values of $\theta_{13} \sim 8^{\circ}, \theta_{23} \sim 45^{\circ}, \Delta m_{13}^{2} \sim 2.4 \times 10^{-3} \mathrm{eV}^{2}$, and the current baseline length of about 1000 $\mathrm{km}$.

LArTPCs offer a unique combination of fine-grained tracking, precise calorimetry, and scalability to very large volumes. These features make the LAr technology well suited for the study of neutrino interactions and searches for rare phenomena. Even though the ability to study the neutrino interactions with LAr technology is superb, the concept is quite simple as depicted in Figure 5.1. It starts with a neutrino interacting with an argon atom inside the TPC volume, which is filled with liquid argon. The particles created in the process of this interaction produce ionization electrons as they quickly travel through the liquid. This ionization is free to drift through the liquid as argon is a noble element. An electric field is applied that separates the ions and electrons. The ionization electrons are drifted towards wires planes oriented an angle to each other. The ionization induces a current on "induction" plane and is collected on the "collection" plane. The wire signal pulses are 
then analyzed. The signal on induction plane is bipolar as electrons induce a current on a wire as they approach and then an opposite sign current as they recede. The signal on the collection plane, on the other hand, is unipolar as the charge approaches the wire and is collected.

Wire plane orientation provides a two dimensional view of an event. Combining the information from both planes together with time allows for three dimensional reconstruction of each event. In addition to timing information from the charge induced and collected, calorimetric information is also obtained as this charge is proportional to the amount of energy deposited by the particle. Scintillation light is also produced in the interaction. ArgoNeuT experiment does not employ photomultiplier tubes to detect it. However, other LArTPC detectors can, and thus they would be able to measure the start time of an interaction more precisely. ArgoNeuT acquires the time of initial neutrino interaction from beam timing information. The implementation of light detection can also serve as a trigger for physics other than the accelerator beam, for example, supernova diffuse or proton decay, and thus can further complement the already superb technique.

In order to achieve a minimum signal attenuation, a great care of electric fields in the region between the drift volume and the induction wire plane, and between the induction and collection wire planes must be taken. For maximum grid electron transparency, the ratio of electric field in region $1, E_{1}$, to electric field in region $2, E_{2}$, for an electron traveling from region 1 to region 2 must be carefully chosen. According to transparency condition, this ratio must be [68]:

$$
\frac{E_{2}}{E_{1}}>\frac{1+\rho}{1-\rho}
$$

where $\rho=2 \pi r / a, \mathrm{r}$ is wire radius and a is the distance between the wires. The equation can be applied for more than just two wire planes. Often, a "shield" plane is also used, in addition to induction and collection planes, separating them from the larger drift region. Its task is to shield the other planes from having a charge induced before the ionization electrons enter the wire plane region. The shield plane must satisfy the following condition in order for the induction and collection planes not to have induced charge from drifting ionization:

$$
\frac{a}{2 \pi s} \ln \left(\frac{a}{2 \pi r}\right) \sim 1
$$




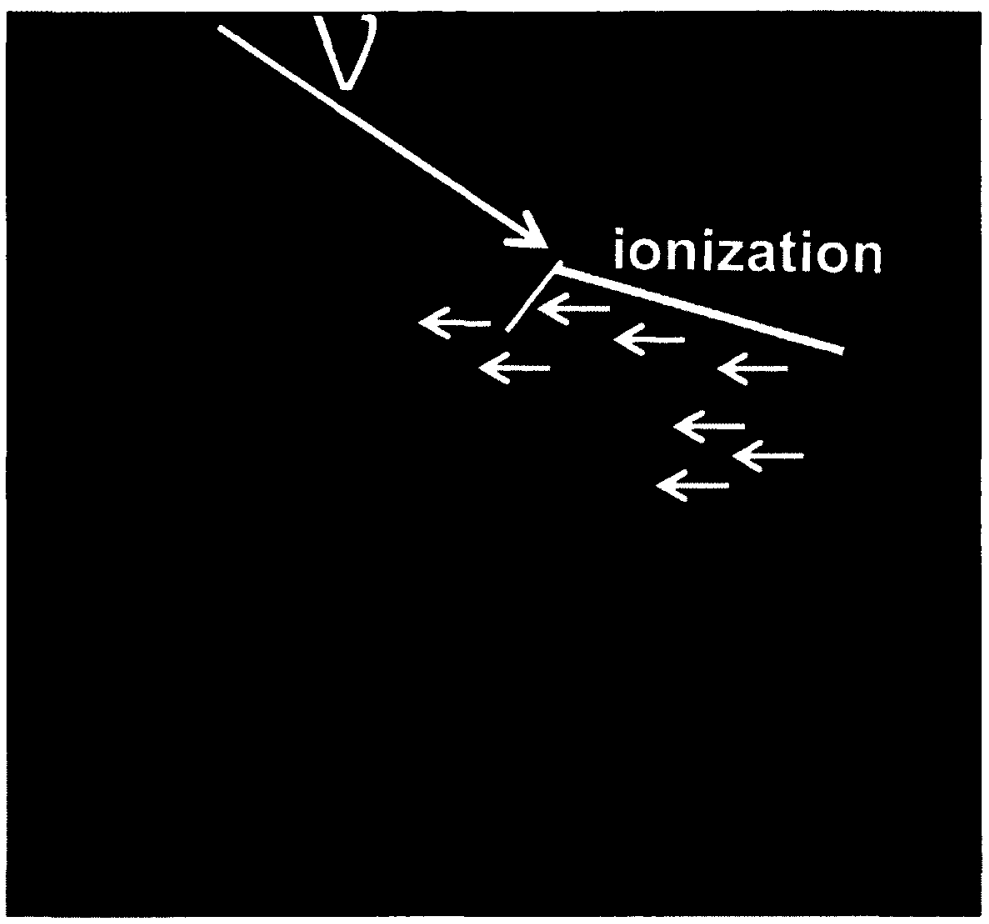

Figure 5.1: The LArTPC concept for neutrino detection showing the TPC filled with liquid argon. A neutrino comes in and interacts with an argon atom. The charged particles produced in the interaction ionize the argon atom. The ionization charge is drifted to the wire planes by the electric field. It induces wire signals on "induction" and "collection" plane, where it is finally collected. Each plane produces a two dimensional view of the event in terms of wire number and time of detection. Combining information from all instrumented planes allows for three dimensional reconstruction of the event.

where $s$ is the distance between the shield plane and the next plane. Another function of the shield plane is to shape the field in the region close to the wire planes. The shield plane can also be instrumented to prove another view of an event. Thus, helping in the three dimensional reconstruction of the event. ArgoNeuT, only instruments induction and collection plane.

The reasoning for choosing argon as the medium and comparison with other stable nobel elements is illustrated in Figure 5.2. Argon is a great medium for neutrino detection because of its large density, short radiation length and high scintillation yield. One can make a similar argument for Krypton and Xenon, however, Argon is far more abundant in the atmosphere and much cheaper. In order to have higher interaction rate liquid is chosen over gas Argon. It also eases the detection due the higher ionization density and 
track reconstruction due to lower electron diffusion. Argon is also a good medium for high voltage application.

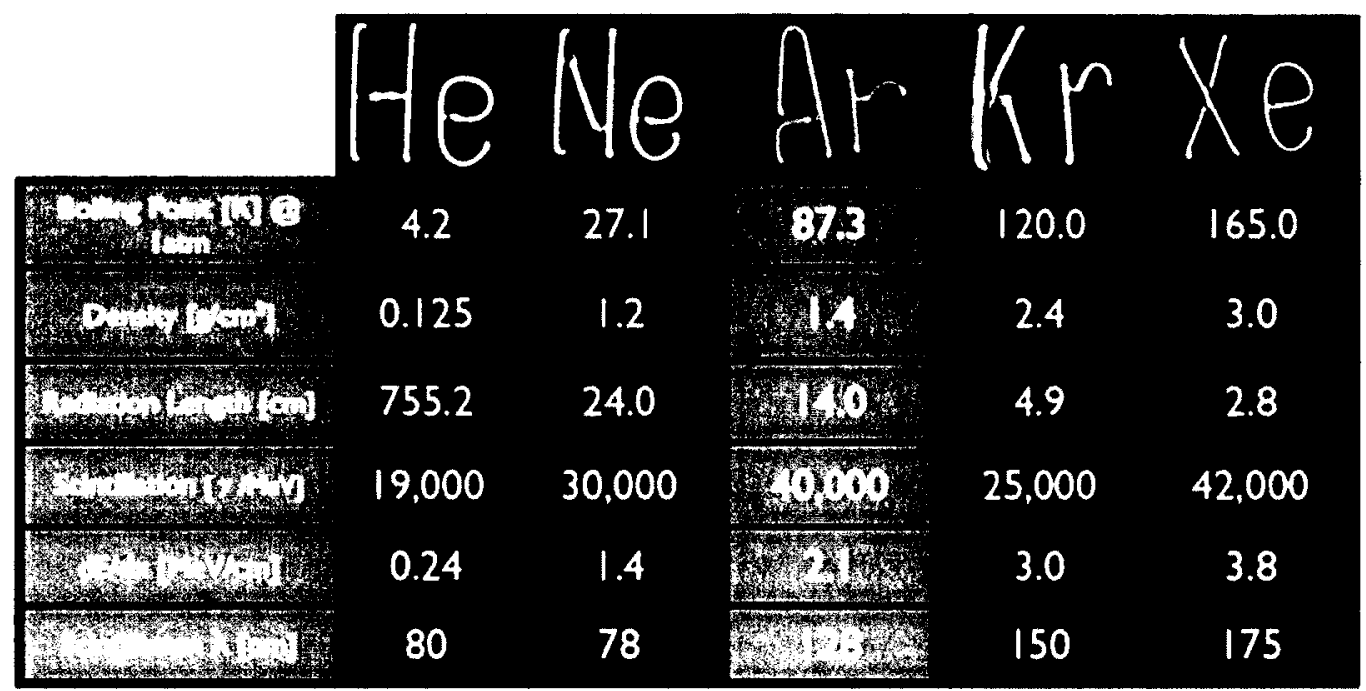

Figure 5.2: Chosen properties of the stable noble elements. 


\section{Event Imagining and Display}

In ArgoNeuT two wire planes, Induction and Collection, are instrumented and provide a 2D image that corresponds to the event projection on a plane. The $\mathrm{x}$-coordinate corresponds to the "wire number" while y to "time ticks". The wire-coordinate runs from 1-240 and time tick of the signal digitization from 1-2048 on each plane. A schematic depicting the wire plane geometry and the reference coordinate frames is shown in Figure 6.1. The induction plane coordinates are $(v, t)$ and $(w, t)$ for the collection plane. Time coordinate is the same for both planes and wire coordinates lie along the wire pitch directions. Each plane contains a $2 \mathrm{D}$ image of the ionization tracks in an event. The signal pulse amplitude is color-coded and corresponds to the local ionization charge release. The 3D image is obtained by combining information from the two 2D views of Induction and collection planes. Some examples of events gathered by ArgoNeuT during the data taking phase are shown in Figures 6.2-6.4. 


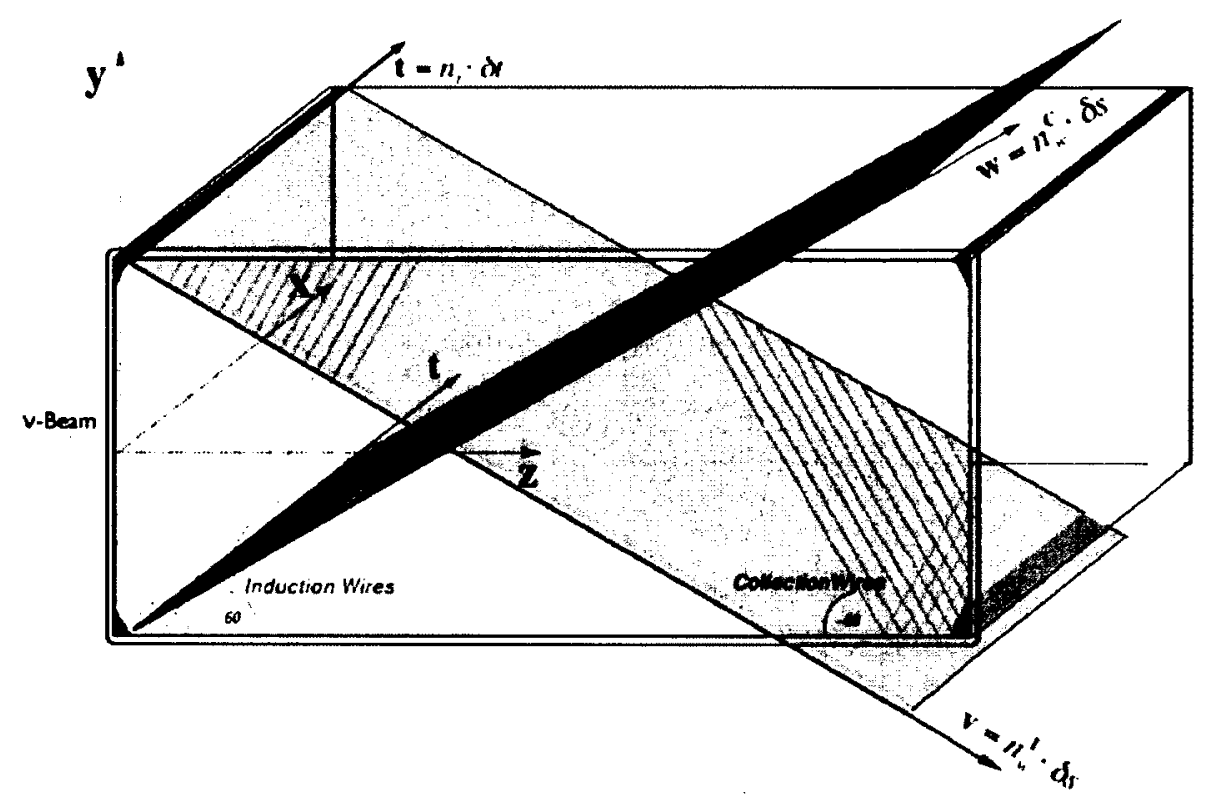

Figure 6.1: A schematic of the ArgoNeuT reference frames used for $2 \mathrm{D}$ and $3 \mathrm{D}$ imaging of the ionization events. The induction plane coordinates are $(v, t)$ and $(w, t)$ for the collection plane and are indicated in terms of wire index $n_{W}^{I, C}$, wire pitch $\delta s$, time tick index $n_{t}$, and sampling time $\delta t$. 

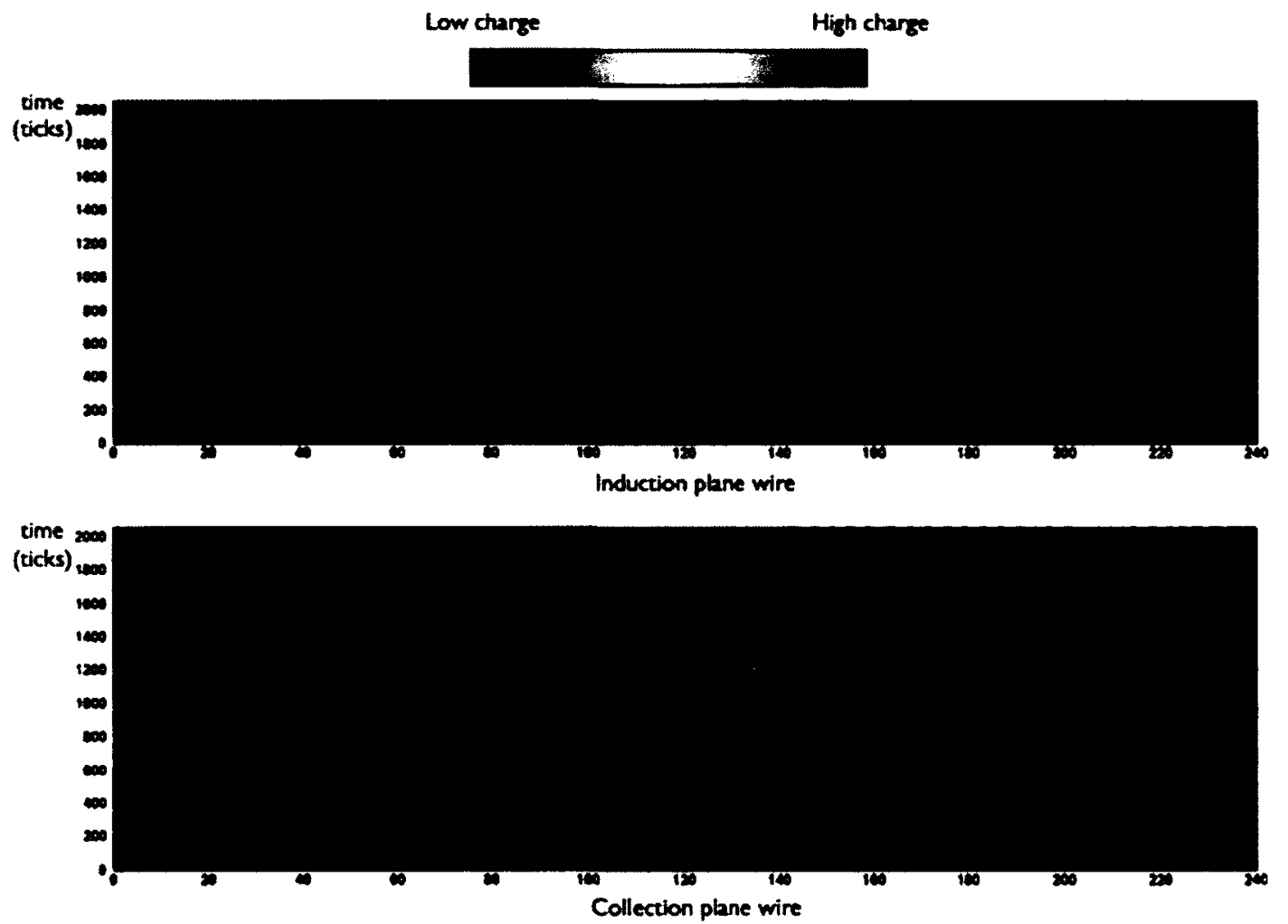

Figure 6.2: A CC $\nu_{\mu}$ candidate event with two tracks clearly visible. The longest track corresponds to a MIP-like particle escaping ArgoNeuT TPC. The short track is a more densely ionizing particle as seen by the red trail. 

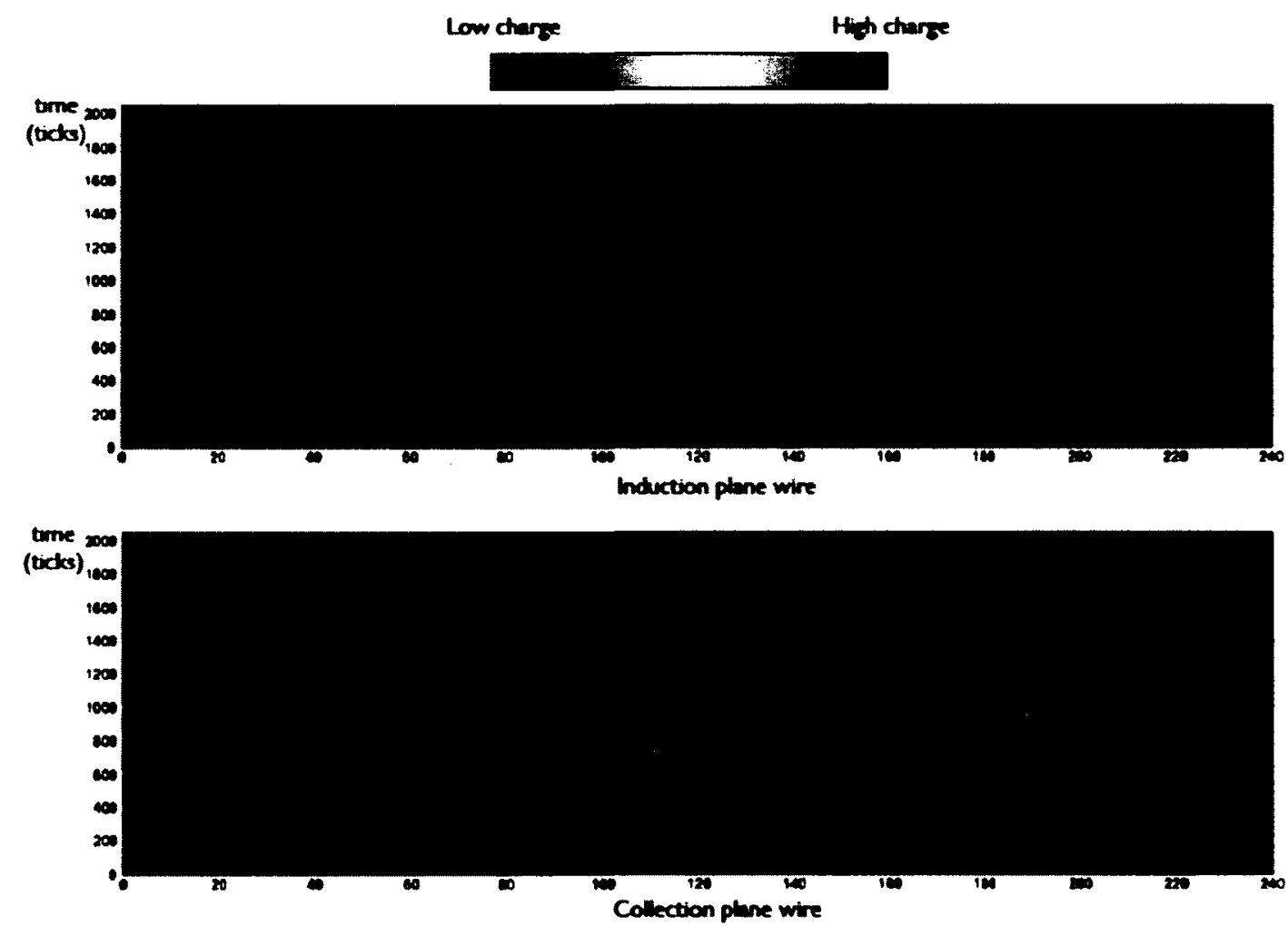

Figure 6.3: A CC $\nu_{\mu}$ candidate event with four tracks. The longest two track correspond to a MIP-like particle escaping ArgoNeuT TPC. The two short tracks are highly ionizing (proton-like) and associated with the interaction vertex. 

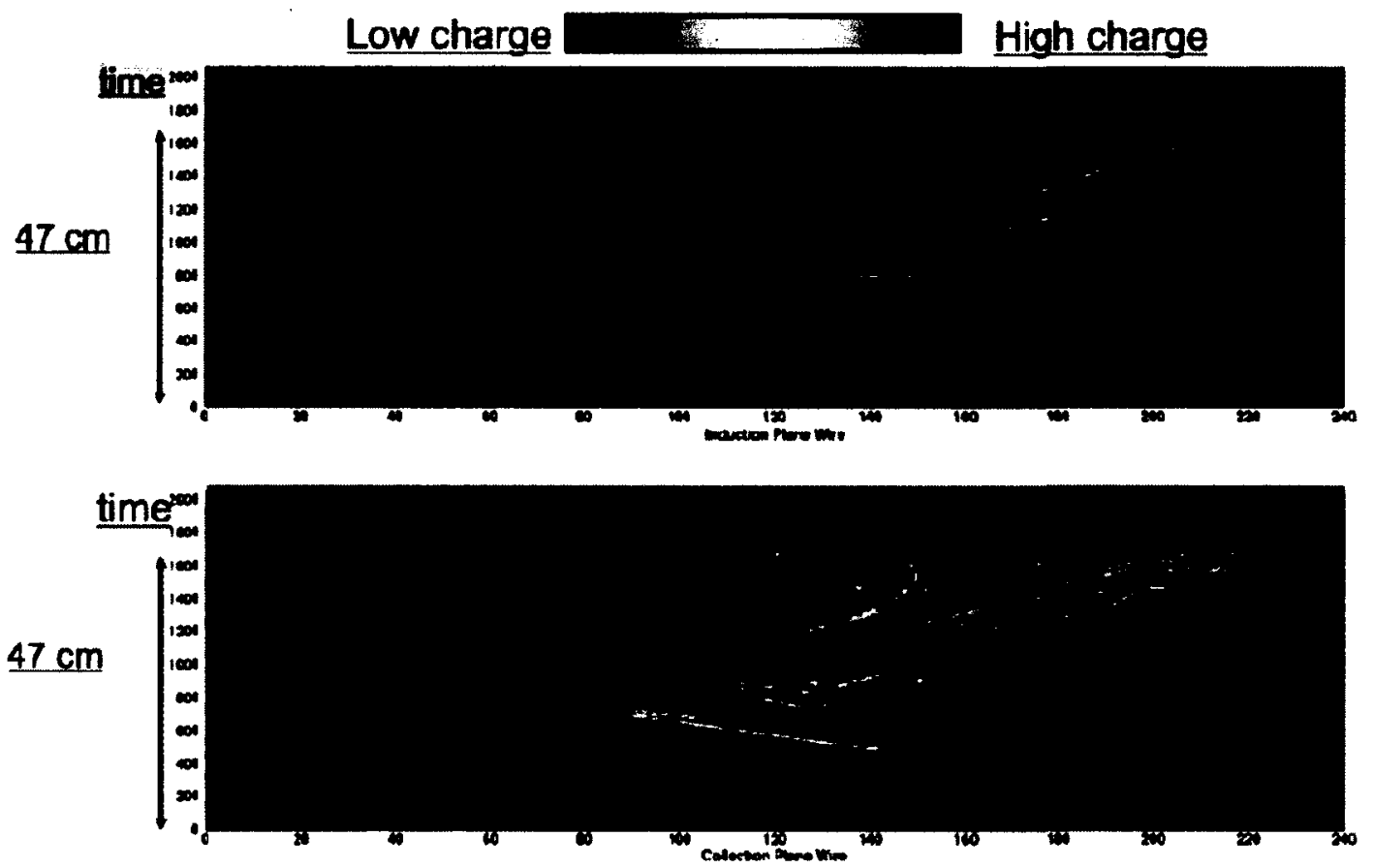

Figure 6.4: A neutrino interaction featuring four well separated showers visible in both planes, and three other tracks originating at the interaction vertex. 


\section{$7 \quad$ Future of the LArTPCs in the US}

\subsection{MicroBooNE}

The next step after ArgoNeuT is the MicroBooNE detector, currently in the construction phase at Fermilab. It is a state-of-the-art 170 ton liquid argon TPC, which will run in the Booster Neutrino Beam at Fermilab in 2014. The TPC has three wire planes instrumented with a total of 8256 channels, and an optical system with cryogenic photomultiplier tubes (PMTs). MicroBooNE's size allows for reaching significant R\&D milestone for a phased program in the United States to multi-kiloton scale LArTPCs. The current plan is to run for about 2-3 years in neutrino mode for a total of $6.6 \times 10^{20}$ protons on target. The detector will be located on surface in the Fermilab Booster Neutrino Beamline. The TPC drift length is $2.5 \mathrm{~m}$ and there are three instrumented wire planes aligned at $90,+30$, and - 30 degrees with respect to the neutrino beam direction. The comparison of MicroBooNE's main detector specification with ArgoNeuT's is shown in Table 7.1. The current installations of the TPC and it's relative size can be seen in Figure 7.2.

\begin{tabular}{lcc}
\hline & ArgoNeuT & MicroBooNE \\
\hline Cryostat volume & $0.7 \mathrm{ton}$ & $150 \mathrm{ton}$ \\
TPC volume & $0.25 \mathrm{ton}$ & $86 \mathrm{ton}$ \\
Max. drift length & $0.5 \mathrm{~m}$ & $2.5 \mathrm{~m}$ \\
Electronics & JFET $(293 \mathrm{~K})$ & CMOS $(87 \mathrm{~K})$ \\
\# of channel & 480 & $\sim 9,000$ \\
Wire pitch & $4 \mathrm{~mm}$ & $3 \mathrm{~mm}$ \\
\# of wire plane & 2 & 3 \\
Light collection & none & 30 of 8"PMT \\
\hline
\end{tabular}

Figure 7.1: Comparison of ArgoNeuT and MicroBooNE detector specifications.

MicroBooNE has many R\&D goals, they include: 


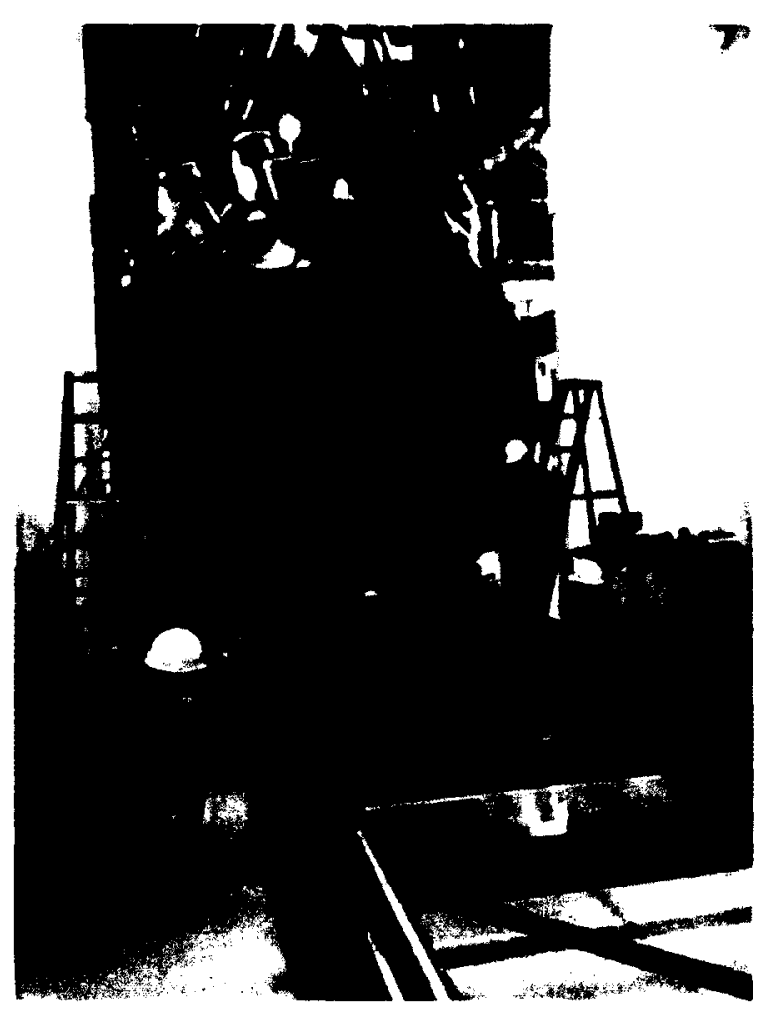

Figure 7.2: Installations of MicroBooNE TPC. The size of the detector with respect to people can be easily referred.

- Demonstration of efficient electron/photon identification

- Keeping the argon pure in a large detector

- Development and demonstration of cold electronics

- Efficient collection of the beam-off data for background studies

- Further development of data analysis tools and reconstruction software

- Providing a cost scaling model for larger future LArTPCs

MicroBooNE has great physics goals, the main ones include:

- Investigation of MiniBooNE "low energy excess" of $\nu_{e}$ events reported in neutrino mode, as well as the excess seen at low and intermediate energies in anti-neutrino mode $[86,87]$ 
- Measurements of neutrino cross sections on argon important to future oscillations and proton decay experiments

- New searches for exotic particles

- Search for proton decay

\subsection{Next Generation Long Baseline Oscillation Experiment}

The Long Baseline Neutrino Experiment (LBNE) is a new high energy physics project currently in its design phase. The idea is to use a powerful beam of protons from the Fermilab Main Injector at Fermilab in order to generate an intense beam of muon neutrinos. The neutrinos will then travel more than a thousand kilometers underground to the Sanford Underground Research Facility in Lead, South Dakota [88]. The detector will be enormous, more than ten times the size of the largest LHC detector. The detector will also be used to study solar and atmospheric neutrinos. Furthermore, it will perform studies of proton decay as well as neutrinos from supernovae. The hope of the LBNE collaboration is to observe $\mathrm{CP}$ violation in neutrinos, which could help to explain the matter/antimatter asymmetry in the universe. 


\section{PART III}

The ArgoNeuT Experiment 


\section{The NuMI Neutrino Beam}

One of the discovery tools in neutrino physics is a neutrino beam from a high energy proton accelerator. Neutrinos come from the decays of charged $\pi$ and $\mathrm{K}$ mesons, which in turn come from proton beams striking nuclear targets. In order to control the energy spectrum and type of neutrino flavour beam, a precise selection of the mesons is needed. The decays contributing to the muon neutrino spectrum are:

- $\pi^{ \pm} \rightarrow \mu+\nu_{\mu}$

- $K^{ \pm} \rightarrow \mu+\nu_{\mu}$

- $K_{L}^{0} \rightarrow \pi+\mu+\nu_{\mu}$

- $\mu \rightarrow e+\nu_{e}+\nu_{\mu}$

ArgoNeuT used the Neutrinos at the Main Injector (NuMI) beam at Fermilab, which produced its first neutrinos in 2005. The order of acceleration steps are:

- the $750 \mathrm{keV} \mathrm{H}^{-}$ion source

- the $400 \mathrm{MeV} \mathrm{H}^{-}$ion linac

- the $8 \mathrm{GeV}$ Booster proton synchrotron

- the $120 \mathrm{GeV}$ Main Injector proton synchrotron

The first step is the ionization of diatomic hydrogen into $\mathrm{H}^{-}$ions, which are then accelerated by a Cockcroft-Walton and reach energy of $750 \mathrm{keV}$ [69]. The ions are now ready to be fed to a Linac and accelerated using a series of drift tubes. Next, the ions pass 
through Side-Coupled Linacs, which accelerate them to energy of $400 \mathrm{MeV}$ [70]. Then they are injected to Booster, which is a synchrotron of 150 meter in diameter.

The Booster operates at $15 \mathrm{~Hz}$ and accelerates protons to $8 \mathrm{GeV}$. The circumference of the Booster is about $1 / 7$ th that of the Main Injector (MI) and six batches of protons from the Booster fill its circumference. An operational mode called slip-stacking is employed. In this mode five batches of protons are injected from the Booster into MI then another bunch is slowly "slipped" until it lines up with the others. The process is repeated four times and the last 11th bunch fills the remaining gap. During the Tevatron running nine were used to make neutrinos while two were used for anti-protons.

The protons from the Booster are directly taken to the Main Injector and the filling takes about $0.73 \mathrm{~s}(1 / 15 \times 11$ batches $)$. Then accelerating them from 8 to $120 \mathrm{GeV}$ takes about $1.5 \mathrm{~s}$ so the whole cycle is 2.2 seconds. They are bent downward by $58 \mathrm{mrad}$ toward Soudan, MN, the site of the MINOS remote detector. The beam line is designed to accept $4 \times 10^{13}$ protons per pulse. The repetition rate is $0.53 \mathrm{~Hz}$, giving about $4 \times 10^{20}$ protons on target per year.

Production of neutrinos begins with $120 \mathrm{GeV}$ protons striking graphite target [71, 72]. There are two magnetic horns whose task is to focus resulting pions and kaons. A schematic of two horns used for the NuMI beam is shown in Figure 8.1. Together, the two horns act as a lens system. The horns can be modified by changing the current and its polarity, which flips the sign of the magnetic field and consequently changes the type of charge being focused. The focal point of the horn system is adjusted by changing the magnitude of the current, which in turn determines the magnetic field within the horns. When operating in neutrino mode positive mesons are focused, and the opposite sign mesons when operating in anti-neutrino mode with horns operating at $185 \mathrm{kA}$. The relative placement of the two horns and the target optimizes the momentum focus for pions.

The mesons and tertiary muons decay in a $675 \mathrm{~m}$ long, $2 \mathrm{~m}$ diameter steel decay pipe. This length corresponds to approximately the decay length of a $10 \mathrm{GeV}$ pion. Muons, protons, undecayed pions and kaons pass through a Hadron Monitor. It consists of an array of helium ionization chambers, which measure the ionization created by charged particles. This detector makes sure that the beam functions correctly. At the end of the decay pipe there is a hadron absorber that stops any remaining hadrons. What is left are neutrinos and muons. Since muons are minimum ionizing particles in the few $\mathrm{GeV}$ range, most of them were not stopped. After the Hadron Absorber a series of three Muon Monitors are 
stationed and are spread over a large area. In order to reach each successive monitor their energy threshold must increase as they pass greater amount of rock. This allows for the measurement of muon energy spectrum. The information is used to predict pion and kaon spectrum and eventually the expected neutrino and anti-neutrino energy spectrum. The remaining muons are stopped by about $250 \mathrm{~m}$ of rock and only neutrinos remain in the beam and pass to the Near Detector Hall. Pre-target and post-target beamline is shown in Figure 8.2.

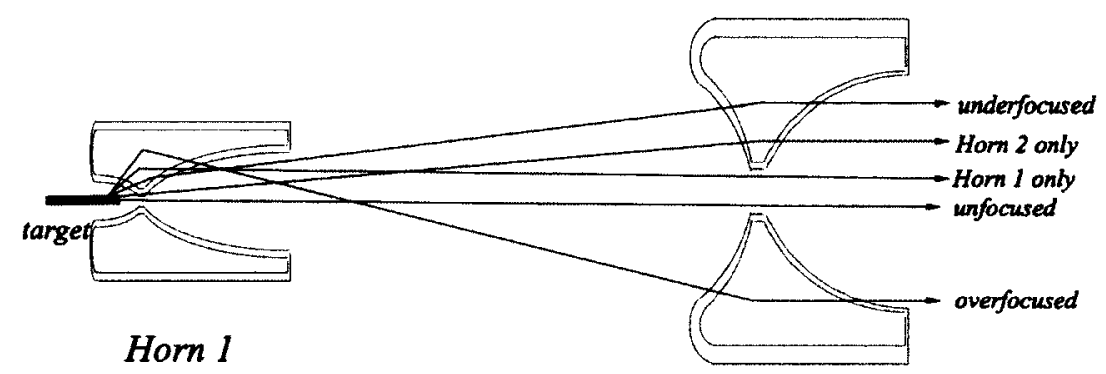

Horn 2

Figure 8.1: Two-lens focusing system for the NuMI line. The second horn improves the collection efficiency of partciles coming from the first horn that were over- or underfocused by the first horn.
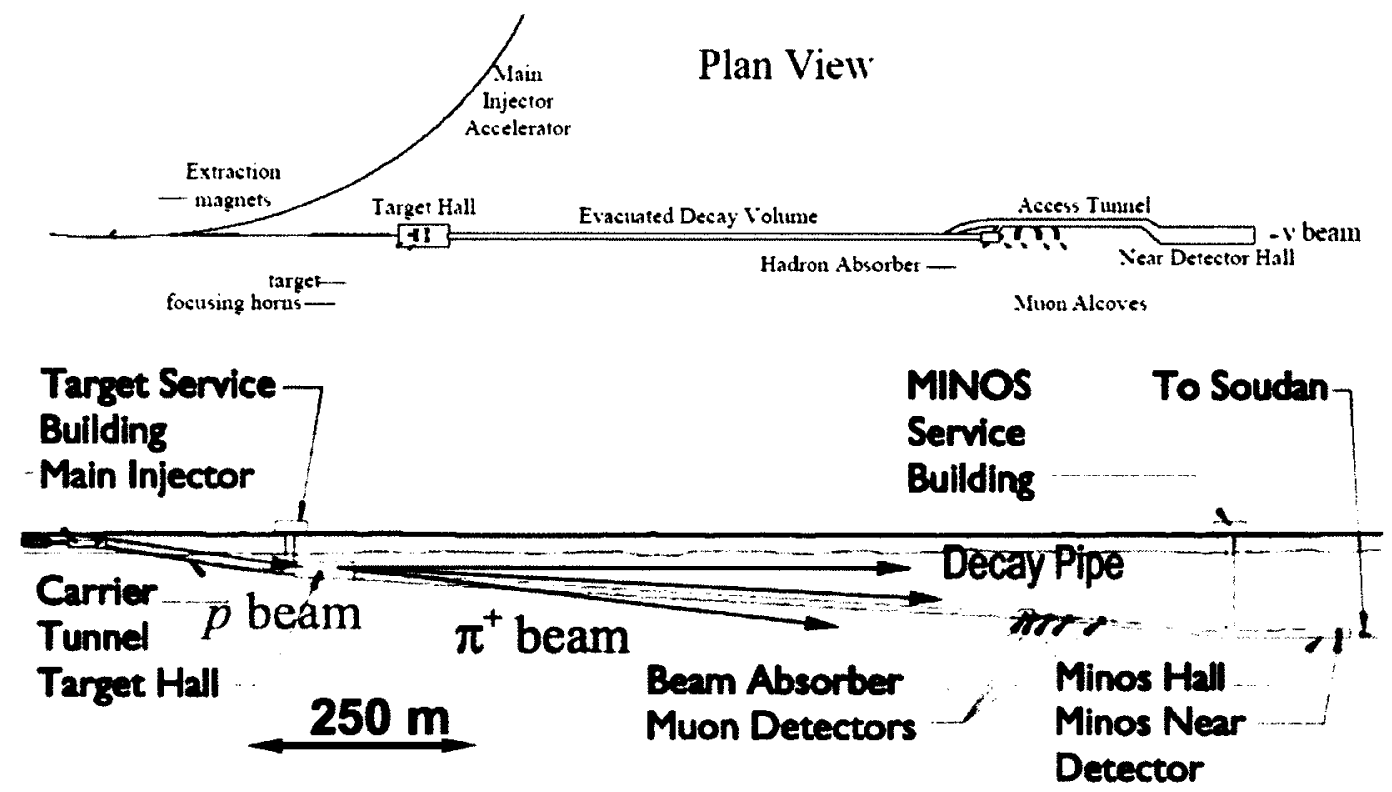

Figure 8.2: Plain and elevation view of the NuMI beam. ArgoNeuT detector was located right in front of MINOS Near Detector in the "near detector hall". 
There are three energy modes in which NuMI can operate: low, medium and high. The entire ArgoneuT run was in the low energy mode. The neutrino spectra from several target position settings are shown in Figure 8.3. The composition of the NuMI beam in neutrino mode is: $91.7 \% \nu_{\mu}, 7 \% \bar{\nu}_{\mu}$ and $1.3 \% \nu_{e}+\bar{\nu}_{e}$. In anti-neutrino mode, reverse current, the beam consists of: $39.9 \% \bar{\nu}_{\mu}, 58.1 \% \nu_{\mu}$ and $2 \% \nu_{e}+\bar{\nu}_{e}$ [73]. One has to remember that these figures can be misleading as the composition is energy and angle dependent.

\section{0}

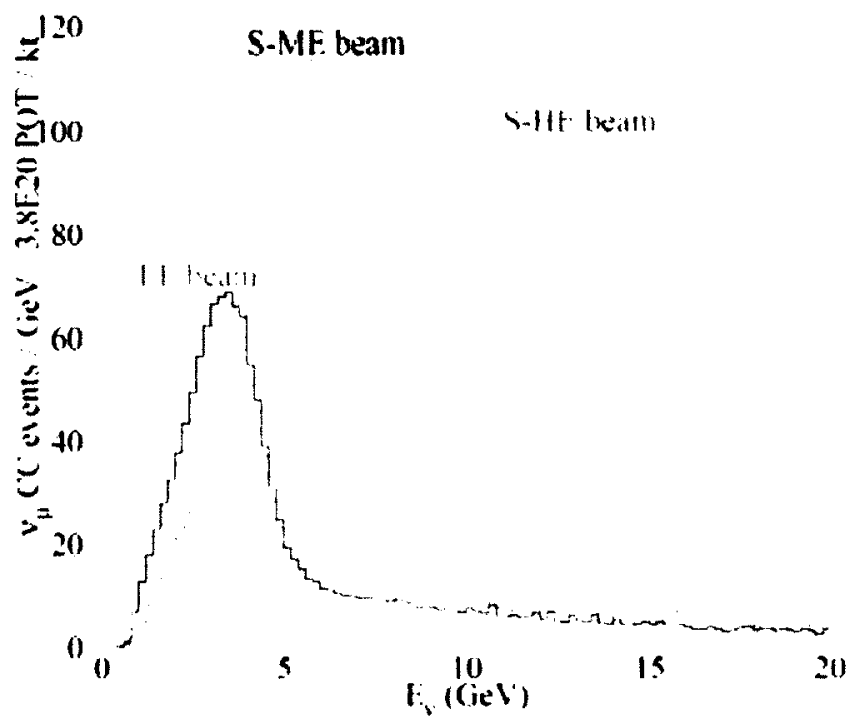

Figure 8.3: Neutrino beam energy spectra with the target in its nominal position inside the horn, "low energy" or retracted $1 \mathrm{~m}$ ("medium energy") or $2.5 \mathrm{~m}$ ("high energy"). 


\section{$9 \quad$ ArgoNeuT Hardware}

The main part of ArgoNeuT detector is the Time Projection Chamber (TPC). The anode of the TPC consists of three parallel wire planes that have wires placed at different angles for each plane. The opposite and parallel side to the anode is the cathode plane. The TPC is subject to a uniform electric field between the cathode and anode planes. The TPC with its field-shaping system is placed in a vacuum insulated cryostat for ultra-pure liquid argon containment.

The electrical connection of the wires to the read-out electronics are provided by feedthroughs on an exit flange on top of the cryostat. The analog readout electronics consists of preamplifier together with the high- and low-pass filters. The wire signals are digitized and recorded at each NuMI beam spill trigger. In order to maintain a constant, pure volume of the liquid argon, ArgoNeuT employes a closed-loop recirculation and purification system. The argon that boils off goes to a cryocooler, mounted above the cryostat, which condenses it back to the liquid state. Purification is achieved by directing the re-condensed argon liquid through a filter that cleans it from impurities, such as oxygen and water. A picture of the ArgoNeuT detector and its location is shown in Figure 9.1. The subsections below give details about the main components.

\subsection{Cryostat and Cryocooler}

The liquid argon is contained in a cryostat consisting of inner and outer vessels. It has cylindrical shape with convex end-caps at each end for both inner and outer cryostats as shown in Figure 9.2. The main axis of the cryostat is horizontal and parallel to the beam. The length of the inner cryostat is $130 \mathrm{~cm}$ and its diameter is $76.2 \mathrm{~cm}$. This volume 

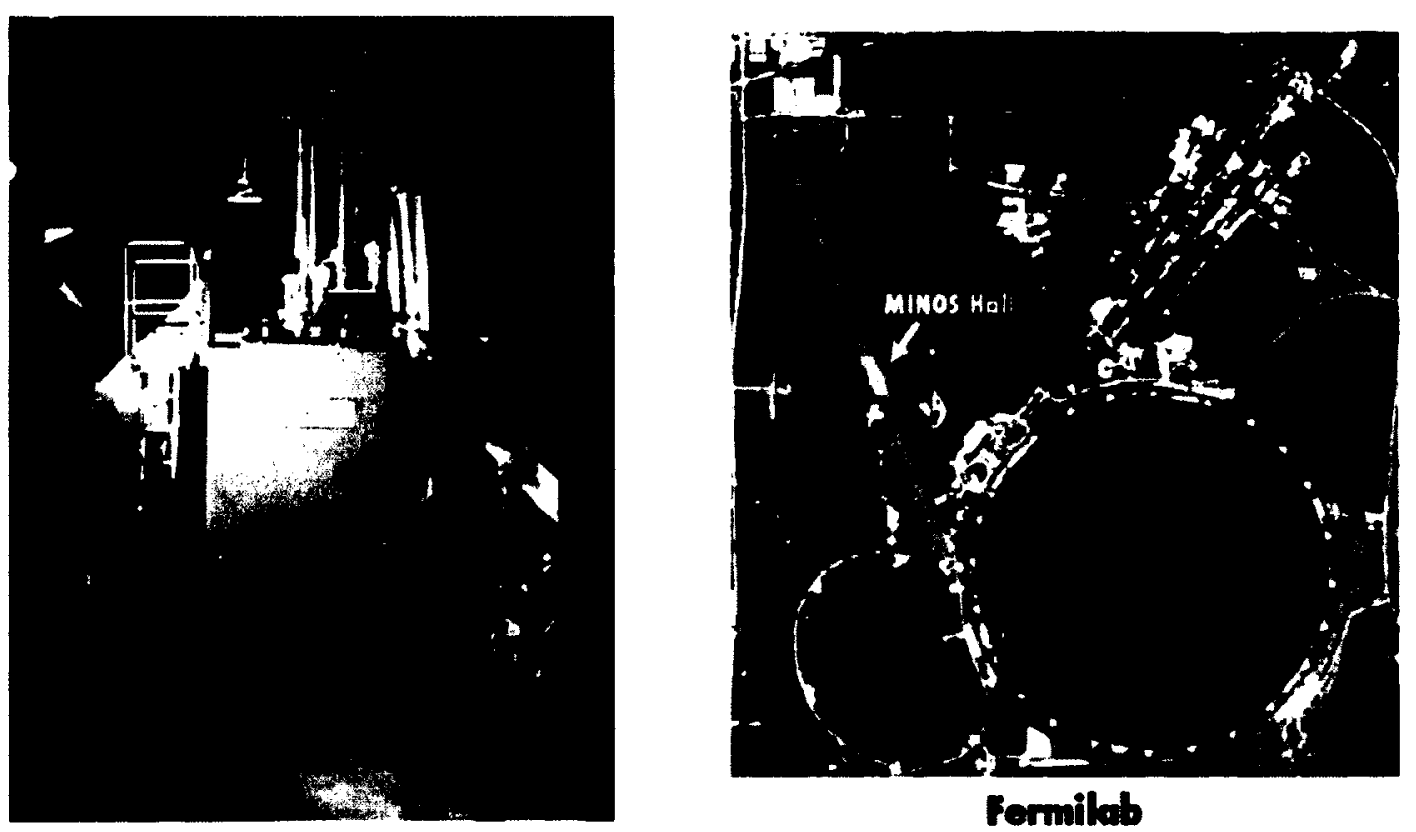

Figure 9.1: [Left] ArgoNeuT detector in the neutrino beam line placed in front of MINOS Near Detector. The cryostat is placed in the orange box, whose purpose is to contain the liquid in case of a major spill. [Right] Aerial view of the Fermilab with MINOS Near Detector Hall pointed out.

corresponds to about $550 \mathrm{~L}(0.76 \mathrm{t})$ of liquid argon. The outer cryostat has length of 163 $\mathrm{cm}$ and diameter of $106.7 \mathrm{~cm}$. The end-caps provide the access to the internal volume for the detector installation.

The cryostat has a wide neck composed of a partially vacuum jacketed chimney formed by two coaxial stainless-steel, straight-joints assembly. The chimney is located on the top of the cryostat. Its function is to provide access for signal cables from the TPC, internal instrumentation, as well as the pipes serving in the recirculation and high voltage feedthrough.

In order to keep the argon in liquid state at constant temperature of $88 \mathrm{~K}$ inside the cryostat, the cryogenic system is based on a commercial single stage cryocooler with high cooling capacity. The compressor package and the cold head expander are separated and connected with flexible lines. The water cooled compressor package provides compressed helium for the cold head inside of which the helium expands in order to create cryogenic temperatures.

A copper heat exchanger is sealed inside a small vacuum insulated vessel connected to the cryostat by a four-pipe vertical pathway. The argon that boils off goes vertically 


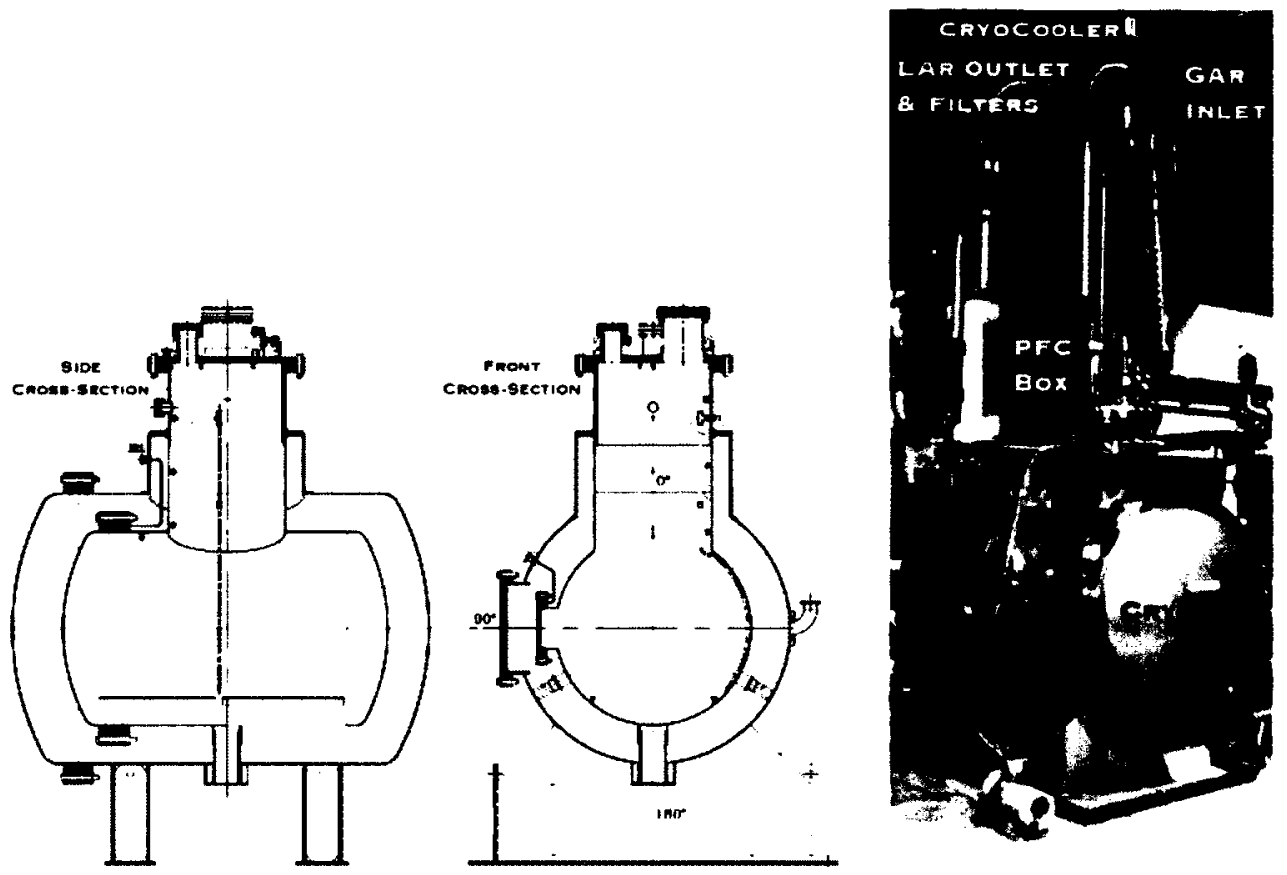

Figure 9.2: [Left] ArgoNeuT cryostat cross-section views. The drawings show the inner and outer vessels, end-caps, and chimney on the top. [Right] Picture of the cryostat taken during the installation.

through one pipe and is recondensed inside the heat exchanger vessel. The argon in the liquid state then travels through one of the other three pipes and returns back to the liquid volume at the bottom of the cryostat. Two of these three passes contain argon filters and the other one is a bypass pipe. During the operation the bypass is closed and the argon cycle uses one filter. The condensed liquid argon enters the cryostat through a vacuum isolated pump.

There are a number of instrumented temperature and pressure sensors on the inner and outer vessels of the cryostat and the cryocooler. Together with the heaters throughout the volume they compose feedback loop. Slow-control software controls this loop by keeping a constant 2.0 psig pressure in the gas pocket above the liquid. If the pressure lowers below the set value, heaters attached to the cryocooler kick in and the net cooling power of the the device is lowered. If the pressure increases above the set value, the heaters are dialed down and the cooling increases. A screenshot of the remotely controllable ArgoNeuT slow-control software is shown in Figure 9.3.

Relief lines are placed in order to allow argon to expand and escape safely. The relief 


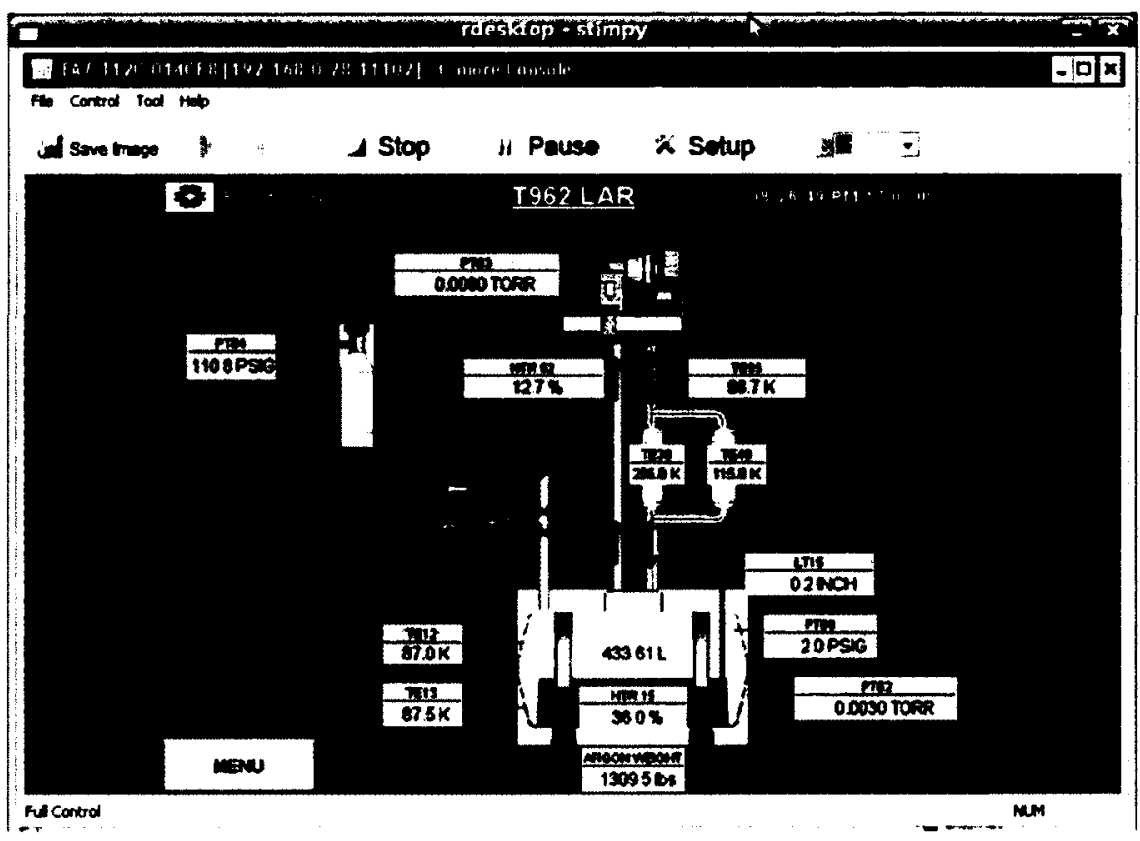

Figure 9.3: A screenshot of the remotely controllable cryo-system monitoring software. The display shows the relevant parameters of the system during operation.

lines lead to a common vent pipe that goes up to the surface from the MINOS Near Detector hall. In case of a major accident, the outer cryostat and the containment vessel, the orange box in Figure 9.1, serve as containers for spilled liquid. The containment vessel is also equipped with oxygen deficiency sensors and fans.

\subsection{Time Projection Chamber}

The ArgoNeuT Time Projection Chamber (TPC) is a rectangular structure $40 \times 47 \times 90 \mathrm{~cm}^{3}$ corresponding to $170 \mathrm{~L}$ of liquid argon. The TPC is positioned inside the inner cryostat. The structure of the TPC is composed of G10, which is a material comprised of woven fiberglass with an epoxy resin binder. The TPC is oriented in such a way that its longest dimension is approximately parallel to the beam. The opposite sides have dimension of $40 \times 90 \mathrm{~cm}^{2}$ and are anode and cathode planes. The drift direction is perpendicular to the beam and horizontal with respect to the ground, it is $47 \mathrm{~cm}$ long from the cathode plane to the innermost wire plane of the anode system. The anode system consists of three parallel wire planes. The planes are spaced $4 \mathrm{~mm}$ from each other. The cathode is a G10 plain sheet with copper metallization on the inner surface. The wires on the wire planes are made out 
of beryllium-copper alloy and have diameter of $152 \mu \mathrm{m}$. They are strung by a wire-winding machine at a tension of $9.8 \mathrm{~N}$. The wire spacing (pitch) is $4 \mathrm{~mm}$ in all planes.

The first plane, called the "shield plane", consists of 225 parallel wires oriented vertically with respect to the ground and thus perpendicular to the beam axis ( +90 degrees). The wires on the shield plane are all equal in length of $40 \mathrm{~cm}$ and stretched from top to bottom of the frame. The plane is not instrumented for readout purposes unlike the other planes. Its purpose is to shape the electric field near the wire-plane and shield the instrumented planes against induction signals from the ionization charges while they are drifting through the TPC volume. The second plane, called the "induction plane", consists of 240 wires oriented at +60 degrees relative to the beam axis. The signal is produced after the electrons pass the shield plane. The electrons move toward, then across and away from the induction plane producing a bipolar signal. The third plane, called "collection plane", consists of also 240 wires but oriented at -60 degrees relative to the beam axis. The electrons are collected onto the wires of this plane and produce a unipolar current pulse. The wires on induction and collection planes vary in length. 144 wires in those planes are of equal length $(46.2 \mathrm{~cm})$ and stretched between the horizontal edges of the frame. Wires of decreasing length fill up the corners of the wire plane area. The wire relative orientation on the three planes is shown in Figure 9.4.

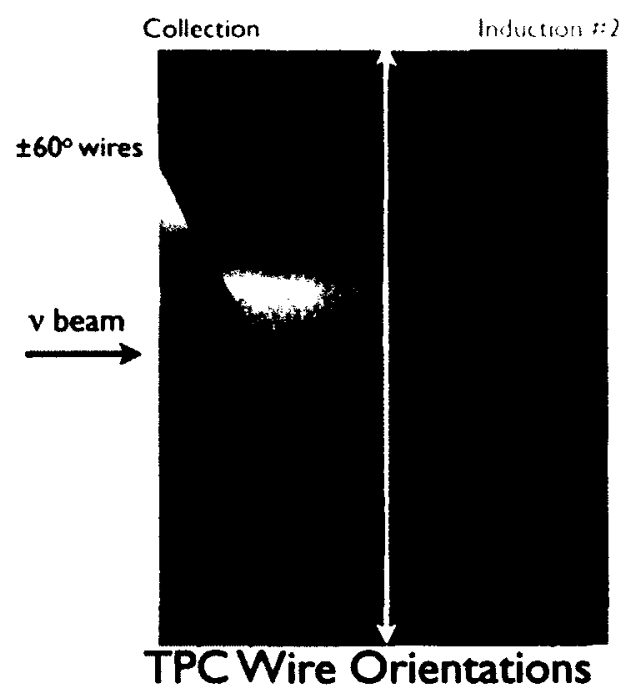

Figure 9.4: Relative orientation of wires on shield, induction and collection planes. Shield plane is not instrumented for the readout.

The electric field throughout the drift region of the $\mathrm{TPC}$ is $500 \mathrm{~V} / \mathrm{cm}$. It is made uniform 
over the drift volume by a field shaping system of electrodes positioned on the boundary surface surrounding the volume between the cathode and anode planes. Four side panels delimiting the TPC box have $1 \mathrm{~cm}$ copper strips placed all the way up the TPC. They are spaced at $1 \mathrm{~cm}$ intervals, forming 23 rectangular rings. An electrical connection of the copper strips is provided by copper tabs soldered in the four corners of the rings. The rings are set at potential linearly decreasing from the cathode to the shield plane and shape the field to be uniform inside and near the edges of the TPC volume. Thus, ionization electrons can move at constant drift velocity. The TPC, which is about to enter the inner vessel of the cryostat can be seen in Figure 9.5.

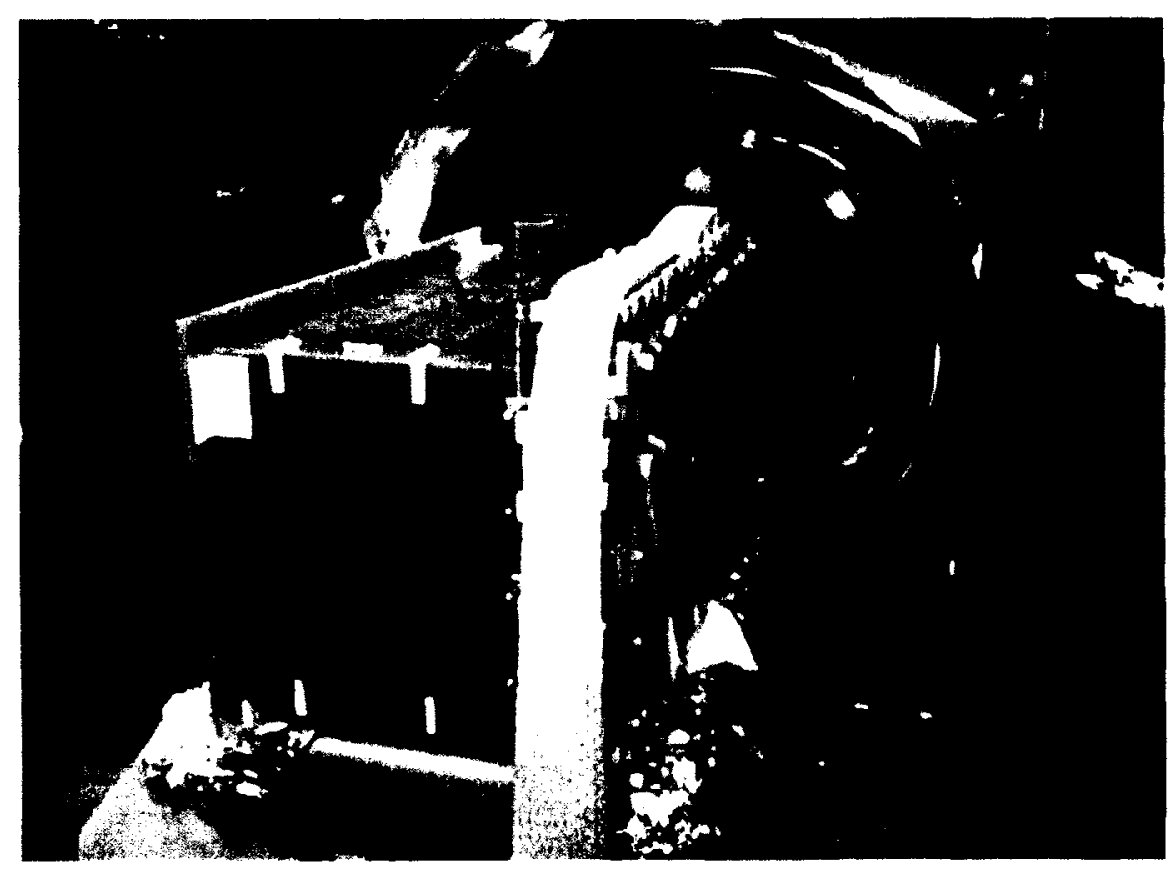

Figure 9.5: The ArgoNeuT TPC about to enter the inner vessel of the cryostat through the removed front-end cap.

\subsection{Electronics Readout}

The electronics readout is structured as a multi-channel waveform recorder that continuously records charge information collected by each wire. The readout chain consists of a series of three electronics cards and boards. They are: the bias voltage distribution card (BVDC), the preamplifier and filter card (PFC-16), and the ADC, circular memory buffer 
and VME readout digitizer module (ADF-2). These active parts are shown in Figure 9.6. The most significant electronics specifications are shown in Table 9.7. The elements are connected by internal readout cables, the readout signal feedthrough card, the external readout cables and pleated foil cables.
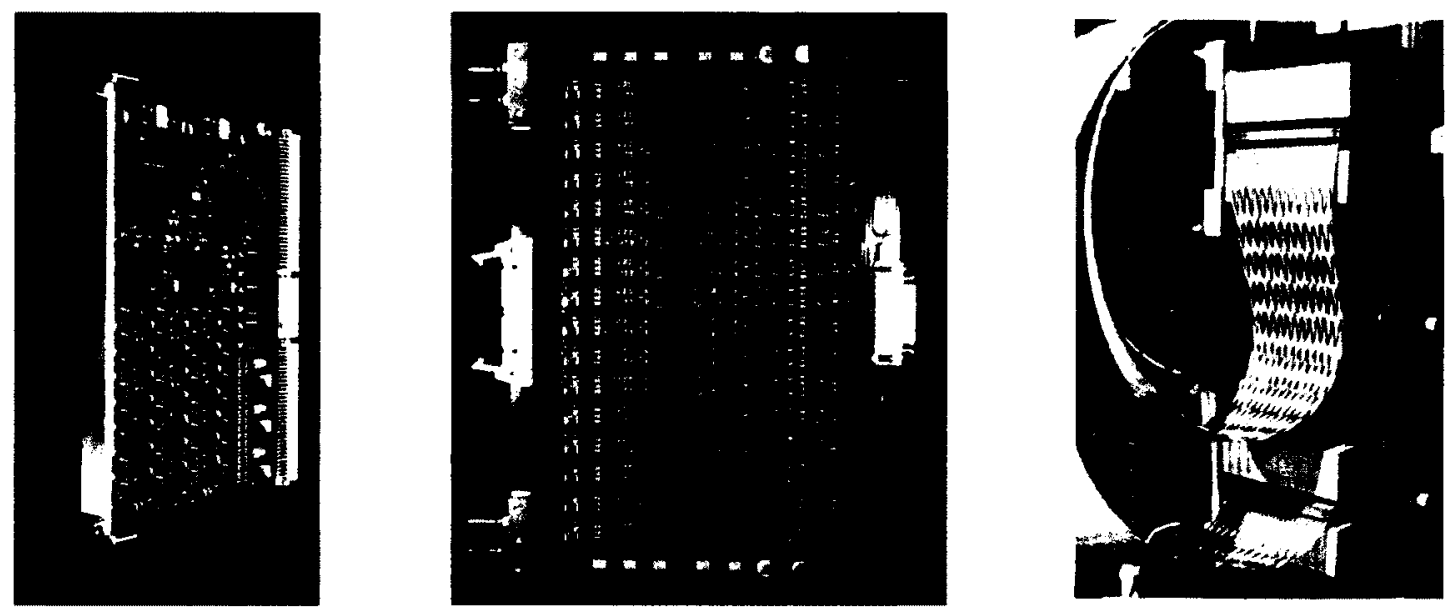

Figure 9.6: [Left] The ADF-2 digitizer card sampling 2048 samples/channel. [Middle] The preamplifier and filter card (PFC). [Right] A set of bias voltage cards (BVDC) that are positioned on the TPC frame.

\begin{tabular}{|c|c|}
\hline $\begin{array}{c}\text { Bias Voltage Distribution Card (BVDC) } \\
\text { \# channels per BVDC } \\
\text { DC Blocking Capacitor }\end{array}$ & $\begin{array}{c}20 \text { units } \\
24 \\
10 \mathrm{nF}(1600 \text { Volt })\end{array}$ \\
\hline Preamplifier and Filler Card (PFC-16) & 30 units \\
\hline \# channels per PFC & 16 \\
\hline PreAmp stage - FET Voltage Gain & $0.5 \mathrm{mV} / \mathrm{rC}$ \\
\hline Shaping \& Filter Stage & "Narrow Gaussian" \\
\hline $\begin{array}{c}\text { 1-pole High Pass } \\
\text { 2-pole Low Pass } \times 2\end{array}$ & $\begin{array}{l}\tau_{\text {diff }}=3 \mu \mathrm{s} ; f_{H P}^{c}=55 \mathrm{kHz} \\
\tau_{\text {in }}=1 \mu \mathrm{s}: f_{L P}^{c}=160 \mathrm{kHz}\end{array}$ \\
\hline Digitizer Module (ADF-2) & 15 units \\
\hline \# channels per ADF-2 & 32 \\
\hline$A D C$ range & 10 bit \\
\hline ADC Gain & $0.1881 \mathrm{ADC} / \mathrm{mV}$ \\
\hline Sampling Time (FPGA) & $\delta t=198 \mathrm{~ns}(5.05 \mathrm{MHz})$ \\
\hline Time ticks per record & $n_{1}=1 \ldots . .2048$ \\
\hline Record Length & $405.5 \mu \mathrm{s}$ \\
\hline \# of Pre-Sampling ticks & $60(11.88 \mu \mathrm{s})$ \\
\hline Electronics Charge Sensitivity & $7.49 \mathrm{ADC} / \mathrm{fC}$ \\
\hline Tot. Capacitance (Det. and Cables) & $230 \mathrm{pF}$ \\
\hline Response to mip (Coll. wires) & $S / N \geq 15$ \\
\hline
\end{tabular}

Figure 9.7: A few relevant electronics readout specifications.

The bias voltage from a noise-filtered external DC power supply is daisy-chained to each 
BVDC. Then it is passed through an additional noise filter and distributed to each sensewire of the TPC through a $100 M \Omega$ isolation and current limiting resistor. The ground bus of the daisy-chain is isolated from the signal ground on the BVDC by $100 M \Omega$ resistors to limit ground-loop currents. A DC blocking capacitor that decouples the DC bias voltage from the AC wire signal is placed between each TPC wire and the readout cable connector. The BVDC with mounted resistors and capacitors are placed on the TPC inside the cryostat and submerged in the liquid argon. Each BVDC services 24 TPC wires and connects the wires to the TPC with two 12-pin connectors. There is no observable effect on electron lifetime due to BVDC [74]. Signals from the BVDC are carried via twenty internal readout flat cables up the chimney of the cryostat to the inner side of the signal feedthrough. The internal readout cables are in argon gas for most of their length.

The gap between the vacuum tight cryostat and the outside world is bridged via readout signal feedthrough. It is custom made with board-mount connectors inside and outside the cryostat. The internal connectors are mounted on a part of the feedthrough printed circuit board that is captured between the flange of a dedicated port on the cryostat chimney and a blanking flange plate, as can be seen in Figure 9.8. After exiting the feedthrough signals are transported to the 16 channel preamplifier and filter card (PFC-16). This card has a preamplifier stage and a shaping/filter stage. The outside of the signal feedthrough flange, the external readout cables and the PFC-16 cards are enclosed in a double RF shielded cage, which is placed on the outside of the cryostat. The cage includes a remote duct cooling system for the preamplifier.
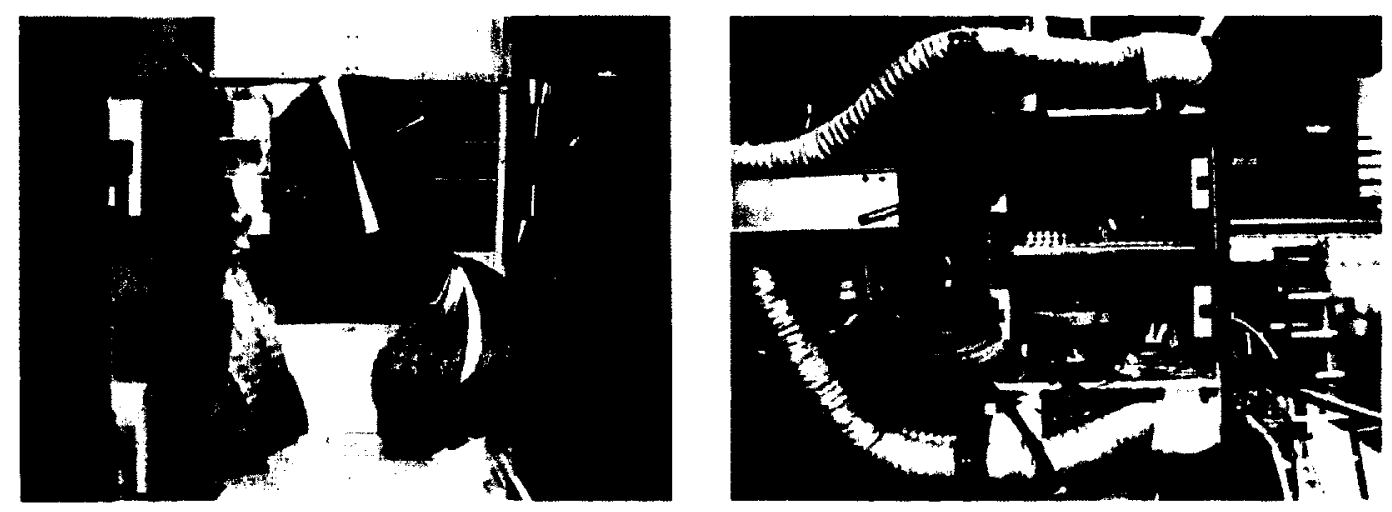

Figure 9.8: The readout signal feedthrough flange inside the RF shielded cage positioned on the outside of the cryostat. The box with PFC in their crate and pleated foil cables to the digitizer board are shown on the right. 
After amplification and filtering stages, the analog signal is transported along pleated foil cables and is digitized by a set of 32-channel ADF-2 modules. The modules were borrowed from D-Zero Run II trigger system spares. Each ADF-2 module has a 10 bit analog-to-digital converter. They are capable of greater than $100 \mathrm{MHz}$ sampling frequency but for ArgoNeuT the ADF-2 sample the preamplifier output every 198 ns and record the digital information for 2048 samples (time ticks). The recorded time window of $405 \mu s$ is greater than the maximum drift time of about $333 \mu \mathrm{s}$. The extra time allows the events to be pre-sampled (60 ticks) and post-sampled. The extra time serves as a debugging tool and noise evaluation.

The PFC-16 and ADF-2 read-out chain is used for all channels of the TPC on both induction and collection planes. The digital signal waveforms are read out by a computer and saved in binary format. The overall sensitivity of the read-out chain is estimated to be $7.6 \mathrm{ADC}$ counts per $1 \mathrm{fC}$ at its input. A more detailed description of the ArgoNeuT electronics readout can be found in [75].

\subsection{Liquid Argon Purification}

A very important task for any liquid argon experiment is to keep the liquid pure so that the ionization electrons can reach the wire planes. A deterrent is the presence of electronegative molecules in the liquid argon. Impurity concentration, for example $e^{-}+\mathrm{O}_{2} \rightarrow \mathrm{O}_{2}^{-}$, is inversely proportional to the electron lifetime $\tau_{e}$ :

$$
\frac{1}{\tau_{e}}=k_{e}\left[O_{2}\right]
$$

The total concentration of electro-negative impurities in liquid argon is often reported in terms of the Oxygen equivalent $\left[\mathrm{O}_{2}\right]$ concentration and expressed in ppb units (partsper-billion). The value of the rate constant $k_{e}$ of the attachment process is dependent from the applied electric field in the TPC volume. For example for $k_{e} \simeq 3.1 p p b^{-1} \mathrm{~ms}^{-1}$ the corresponding electric field is $E F=0.5 \mathrm{kV} / \mathrm{cm}$ [83]. The drift velocity of electrons subject to this field is about $1.6 \mathrm{~mm} / \mu \mathrm{s}$. When impurity concentration is $0.7 \mathrm{ppb}$ (and $\tau_{e}=450 \mu \mathrm{s}$ )* after a drift of $50 \mathrm{~cm}$ (drift time $310 \mu \mathrm{s}$ ) only half of the free ionization electrons make it to the wire planes.

Liquid argon is commercially available but comes with an oxygen-equivalent concentration at the parts-per-million (ppm) level. Therefore, a removal of the impurity content 
is necessary in order to drift the ionization charge through large distances. Filtration techniques should have very high removal efficiency and be fast in order to process large amounts of liquid argon. A few types of oxygen catalyst are commercially available and used in industrial processes in which purified inert gas streams are used. High efficiency removal of oxygen is acquired by oxidation reaction with the metal to form the metal oxide. This process removes the oxygen from the stream and is temperature and pressure dependent. Oxygen storage capacity is an indicator of the removal efficiency, which decreases with growing oxygen retention. Once the filter becomes saturated, the process is restored by a simple regeneration of the metal oxide back to metal form. This reduction process is performed by heating the filter with a flow of hot inert gas containing $2-4 \%$ of $\mathrm{H}_{2}$ by volume. Filters of this type have been custom made and used for liquid argon detectors since the early development of the liquid phase purification technique [79].

The ArgoNeuT filters are made out of activated-copper-coated alumina granules inside of flanged cylindrical stainless-steel cartridges. Liquid argon inlet/outlet flange-fitted lines at the cartridge ends are equipped with vacuum tight cryogenic valves for on-stream connection in the closed loop system. Granules are kept inside the filter by positioning steel disks at both ends of the internal volume while letting liquid argon to flow through. Each cartridge is $61 \mathrm{~cm}$ long with diameter of $6.4 \mathrm{~cm}$, corresponding to about $2 \mathrm{~L}$ of volume. Because of the porous structure of the alumina support the active metal surface, made from copper, available for $\mathrm{O}_{2}$ reaction is very large. The structure also offers $\mathrm{H}_{2} \mathrm{O}$ adsorption capability by molecular trapping. Presence of water at ppm level in the cryostat neck at warm temperature was linked to a decrease in the electron life time. In order to avoid the $\mathrm{H}_{2} \mathrm{O}$ presence, one of the three filters in the recirculation system was partially filled with zeolite molecular sieve to increase water adsorption. The cartridges are wrapped in a $10 \mathrm{~cm}$ thick fiberglass insulation. Two of them are installed as active components of the purification loop pathway, with one open to liquid argon recirculation. The recirculation system can be seen in Figure 9.9. Once a filter gets saturated, the circulation is switched through the other filter and the saturated cartridge is replaced. The cartridge can be used again after the off-line regeneration of the exhausted filter material. A picture of the cartridges downstream of the cryocooler is shown in Figure 9.10.

The argon that boils off goes to the cryocooler, which condenses it back to the liquid state, and in the liquid form it is forced by gravity and pressure to go through the filter. It is returned purified to the bottom of the cryostat. The full content of the liquid argon 


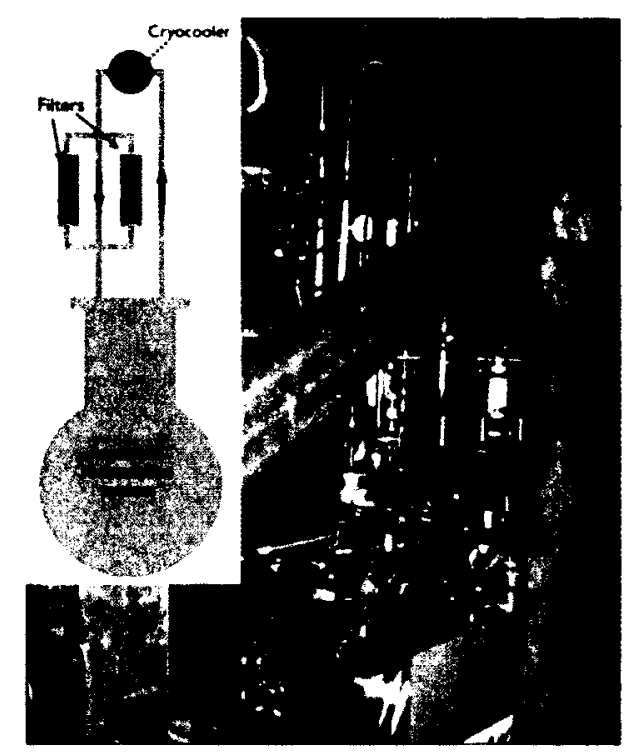

Figure 9.9: The ArgoNeuT recirculation system featuring filters and the cryocooler.

contained in the cryostat ( $550 \mathrm{~L}$ ) gets recirculated every 7-8 days as the recirculation system operates at a flow rate of about $1.6 \mathrm{lt} / \mathrm{hr} / \mathrm{lt}$ (LAr volume per unit of time per volume of filter). A more detailed description of the ArgoNeuT filter method can be found in [80].

\subsection{Measuring Liquid Argon Purity}

In order to monitor the recirculation system, purity monitor was installed inside the inner cryostat, right on top of the TPC. It allowed for a very fast purity determination of the liquid argon even though it was subject to a large systematic uncertainty. It also assisted with filter issues and judging the success of the liquid argon fill. The schematic of the purity monitor concept is shown in Figure 9.11. It starts with directing of light from xenon flash lamp from outside of cryostat to the inside via optical fibers $[81,82]$. The fibers are pointed towards a gold photocathode of the purity monitor. The electrons coming from the photo electric effect are then drifted through the $\sim 100 \mathrm{~V} / \mathrm{cm}$ purity monitor field cage. They are detected $10 \mathrm{~cm}$ away on the anode. In order to determine the attenuation level across the drift distance, the signal on the cathode and anode are evaluated by the use of an oscilloscope. If the purity were infinite the two signals would appear to be equal and opposite after accounting for the cathode and anode's slightly different responses of the electronics readouts. The ArgoNeuT purity monitor is shown in Figure 9.12. 


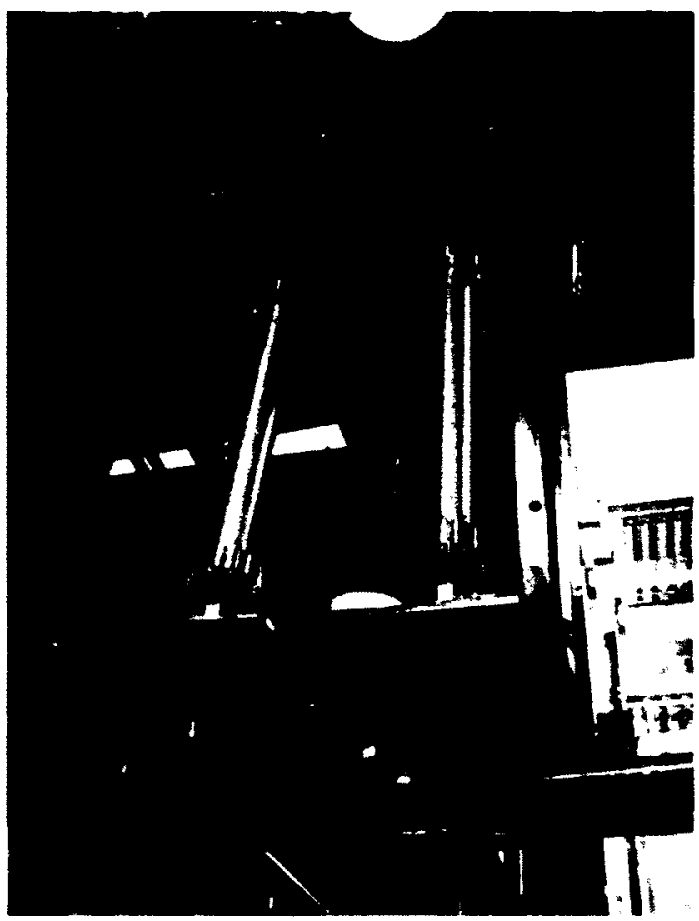

Figure 9.10: The ArgoNeuT filter cartridges serving for liquid argon purification.

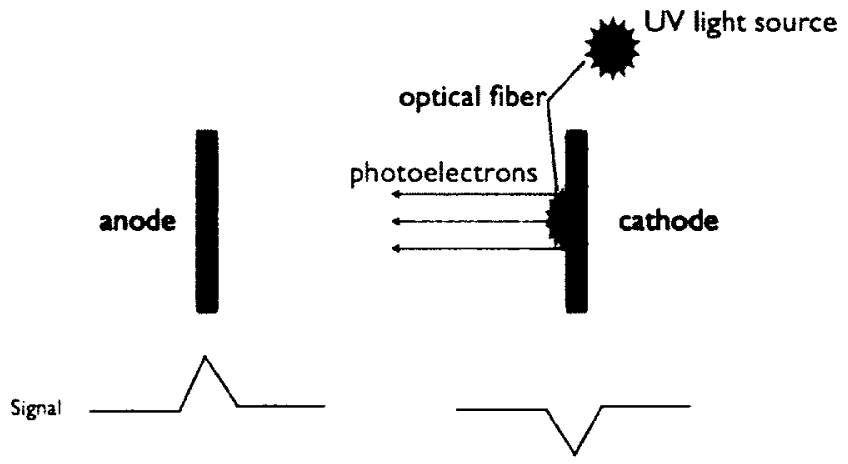

Figure 9.11: The concept of the purity monitor. A measure of purity of liquid argon is determined from comparing the signals of the anode and cathode after electron from photo electric effect drift the full distance between them.

The electron lifetime, $\tau$, has the following dependence

$$
\frac{Q_{a}}{Q_{c}}=e^{-t / \tau}
$$

where $Q_{a}$ and $Q_{c}$ are proportional to the anode and cathode pulse heights. A purity monitor oscilloscope acquisition system was employed to determine the lifetime. A program 


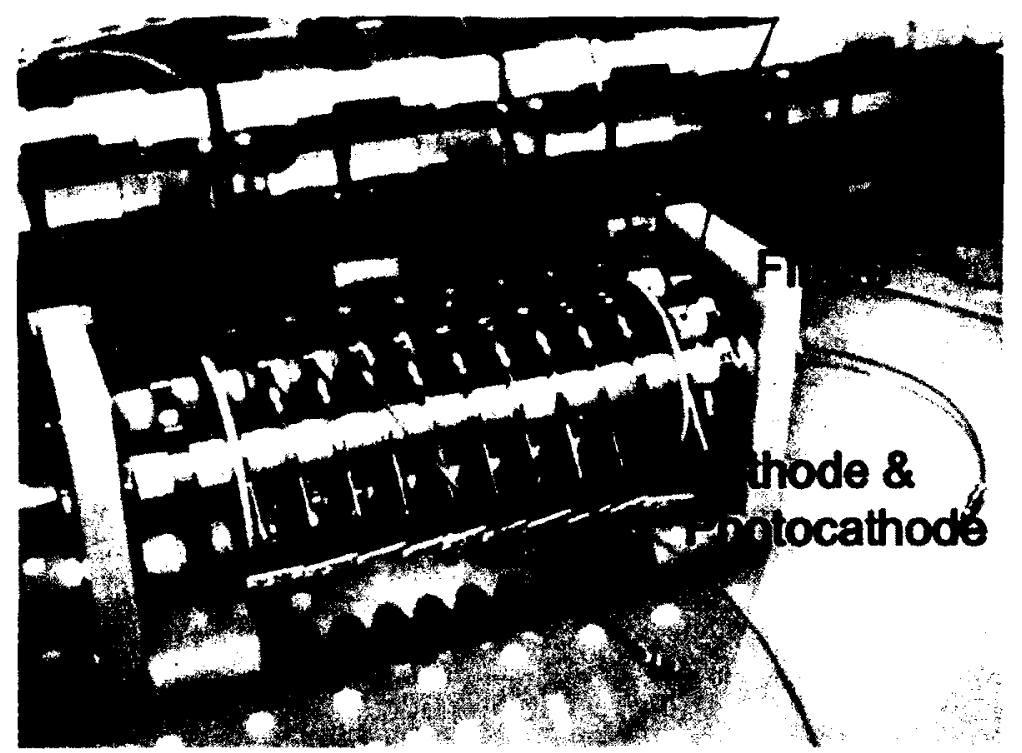

Figure 9.12: The ArgoNeuT purity monitor positioned on top of the TPC.

with algorithm developed for peak finding was used to extract the cathode and anode signal amplitudes. The program then fitted the exponential decay to correct for electronics signal response and then extracted the lifetime. The electronegative impurity concentration can be written as [83] :

$$
\frac{d[e]}{d t}=-k_{s}[S][e]
$$

where $[\mathrm{S}]$ is the electronegative impurity concentration, $k_{s}$ is the electron attachment constant and $[\mathrm{e}]$ is electron concentration. Its solution can be written in a from similar to Equation 9.2 as:

$$
\frac{[e(t)]}{\left[e_{0}\right]}=e^{-k_{s}[S][e]}
$$

After accounting for a few conversion factors to make correspondence between $\left[e_{0}\right],[\mathrm{e}(\mathrm{t})]$ and $Q_{a}, Q_{c}$ respectively, one arrives at:

$$
[S]=\frac{1}{35 k_{\mathrm{s}} \tau}
$$

As an example, an electron lifetime of $750 \mu s$ corresponds to oxygen-equivalent electronegative impurity concentration of $400 \mathrm{ppt}$. 


\subsection{MINOS Near Detector}

The Main Injector Neutrino Oscillation Search (MINOS) is a long-baseline experiment measuring neutrino interactions in the NuMI neutrino beam consisting of two detectors. The near detector is stationed at Fermilab and the far detector is $735 \mathrm{~km}$ downstream in the Soudan Underground Laboratory in Minnesota. ArgoNeuT utilizes only the near detector for obtaining momentum and sign of muons that exited the TPC and entered MINOS Near Detector. Thus, only MINOS Near Detector will be described here.

The MINOS Near Detector is steel-scintillator sampling calorimeter with tracking and energy measurement capabilities. It is located at the end of the NuMI beam facility, 100 $\mathrm{m}$ underground. The cross section of the detector has $3.8 \mathrm{~m} \times 4.8 \mathrm{~m}$ squashed octagon shape as shown in Figure 9.13. The detector has a mass of 980 tons and length of 16.6 m. It uses alternate planes of plastic scintillator strips and toroidally magnetized 2.54 $\mathrm{cm}$ thick steel plates. Between each plane there is either a $1.0 \mathrm{~cm}$ thick scintillator plane and a $2.4 \mathrm{~cm}$ air gap, or a $3.6 \mathrm{~cm}$ air gap. The detector is divided into the upstream and downstream parts. The detector was assembled from 282 steel planes but only 153 of the 282 planes are active. The 120 planes compromise the calorimeter section and are all active to produce a high resolution view of the neutrino interactions. Planes 121-281 comprise the spectrometer section and are used to track muons from neutrino interactions for their momentum determination. The detector is magnetized for charge identification and determination of particle's momentum by measuring the resulting curvature. The current runs through an electromagnetic coil passing through a hole in the detector's center. The coil provides $40 \mathrm{kA}$-turs providing a toroidal magnetic field in the steel between 1-2 $\mathrm{T}$ as a function of radius. The detector has two distinct magnetic field polarities. The forward field focuses negative muons coming from the south, toward the center of the detector. The reverse field focuses positive muons coming from the south, toward the center of the detector.

The MINOS scintillator system consists of extruded polystyrene scintillator strips $4.1 \mathrm{~cm}$ wide, $1 \mathrm{~cm}$ thick and up to $6 \mathrm{~m}$ long. A groove is cut in this surface down one wide side of the strip and a wavelength shifting fiber is glued into the groove to collect the light. A diagram showing the scintillator strip and its parts is shown in Figure 9.14. Clear fiber carries the light from the ends of the strips to the phototubes. At the strip ends another fiber brings in light from a UV LED. Strips are laid side-by-side and bundled into modules. The strips 
in each scintillator layer are rotated by 90 degrees with respect to the previous layer to allow for 3 dimensional track reconstruction. Optical fibers carry the scintillation light to photomultiplier tubes. Wavelength shifting fibers channel the light to the ends of the strips, they minimize self-absorption by absorbing light peaked at $420 \mathrm{~nm}$ and re-emitting it at $470 \mathrm{~nm}$.

The detector is calibrated using an in-situ light injection system and cosmic muons [76]. In order to track the gain changes in the PMTs and electronics LED generated light signals are distributed to all the fibers. The energy deposited by through-going muons is employed to equalize the response of the scintillator strips.

MINOS Near detector is used for the analysis presented in this thesis for complete reconstruction of muons that were not contained by ArgoNeuT and entered MINOS. The muon energy resolution $\Delta E_{\mu} / E_{\mu}$ varies smoothly from $6 \%$ for muons with energy above 1 $\mathrm{GeV}$ where most tracks are contained to about $13 \%$ where mainly curvature measurement is used [77].

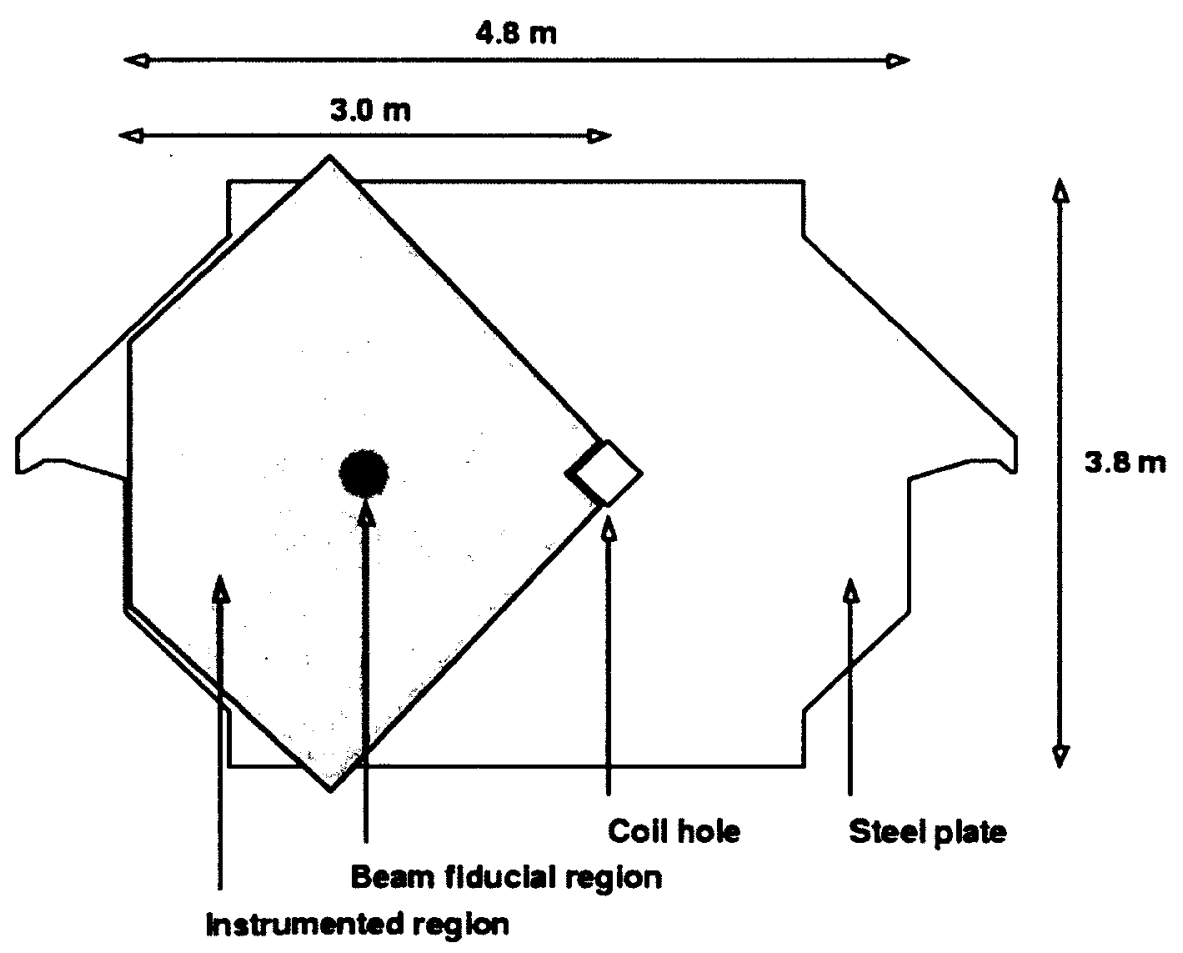

Figure 9.13: A diagram of one plane of MINOS Near Detector $3.8 \mathrm{~m}$ tall and $4.8 \mathrm{~m}$ wide. An electromagnetic coil passes through the hole in the center of each plane. Some planes are fully instrumented while others only on the side hit by the beam as shown by the diamond-shaped region. 


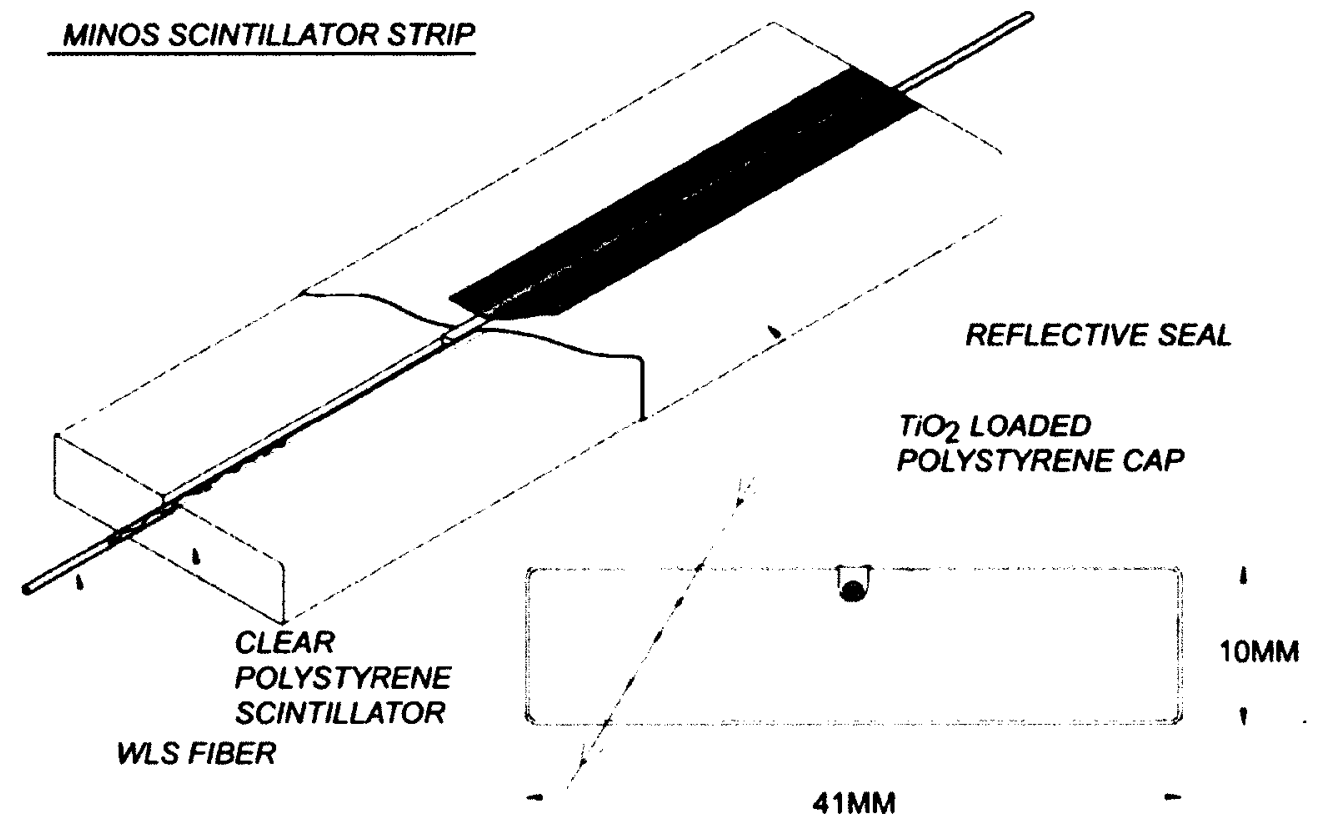

Figure 9.14: The MINOS scintillator strip composition. 


\section{Commissioning and Data Taking}

The ArgoNeuT detector was commissioned for the physics run in the MINOS Near Detector hall at Fermilab in spring 2009. The TPC was inserted into the inner vessel of the cryostat from the front end-cap and then vacuum sealed inside. The argon re-condensation and purification system was leak tested. It was then connected to the cryostat as well as the electronics read-out to the TPC wires through connection to the signal feed-through flanges.

The trigger condition for the data acquisition of the physics run is set in coincidence with the NuMI beam spill signal of $0.5 \mathrm{~Hz}$ rate. The event record contains a time stamp provided by NuMI accelerator complex at each trigger. This information is later used during matching of ArgoNeuT tracks with MINOS Near Detector performed on spill-by-spill basis.

ArgoNeuT physics run began in September 2009 and the data taking lasted for about six months. The entire run, from September 2009 to February 2010, was in "low-energy" NuMI configuration and the accelerator delivered $1.335 \times 10^{20}$ protons on target. The first part of the run was in neutrino mode with $8.5 \times 10^{18}$ protons on target (POT) and the second part in anti-neutrino mode with $1.25 \times 10^{20}$ POT. ArgoNeuT saw an uptime of about $86 \%$ in terms of POT delivered, which included a two week downtime in October 2009 caused by a failure of a commercial component of the cooling system. The ArgoNeuT POT as a function of time is shown in Figure 10.1. With the exclusion of the suspended operation, the uptime would have been around $95 \%$ for the entire physics run. The MINOS Near Detector was operational during the ArgoNeuT run for $90 \%$ of the time.

The events collected by ArgoNeuT can be classified into 3 categories:

- Empty event: Event that does not contain any observable interaction. These events are expected due to the very low neutrino cross section and the small size of ArgoNeuT. They constitute the largest sample of events. 
- Through-going track event: Event containing charged particles produced by neutrino interactions upstream of the ArgoNeuT detector that propagated up to the liquid argon volume. Most of them are muons and their analysis can be found in [84]. They were used for the characterization and monitoring of the significant parameters of the detector, such as the electron drift velocity and the lifetime of the free electron charge.

- Neutrino event candidate: Event where the interaction vertex with one or multiple tracks is contained inside the liquid argon TPC fiducial volume.

Neutrino events can also be classified based on the present signature as:

- Charged-current (CC) $\nu_{\mu}$ candidates with the muon track, often accompanied by some hadrons. The muon track leaves the ArgoNeuT TPC most of the time and is matched with the MINOS Near Detector. A detailed analysis of this sample can be found in $[85]$.

- Charged-current $\nu_{e}$ candidates, which feature an electromagnetic shower developing in the TPC. There might be some additional hadrons present at the interaction vertex.

- Neutral-current (NC) candidates where no muon or electromagnetic shower are associated with the interaction vertex. This sample includes events with electromagnetic showers coming from $\gamma$ conversion following the $\pi^{0}$ decay.

Cross section studies and other measurements can be performed on these samples. There are about $900 \nu \mathrm{CC}$ interactions in the fiducial volume in the neutrino mode configuration. Anti-neutrino running provides a much larger sample: about $4000 \nu \mathrm{CC}$ interactions and $3500 \bar{\nu}$ CC interactions. Some examples of events collected by ArgoNeuT will be shown in the next section. 


\section{ArgoNeuT POT delivered and accumulated}

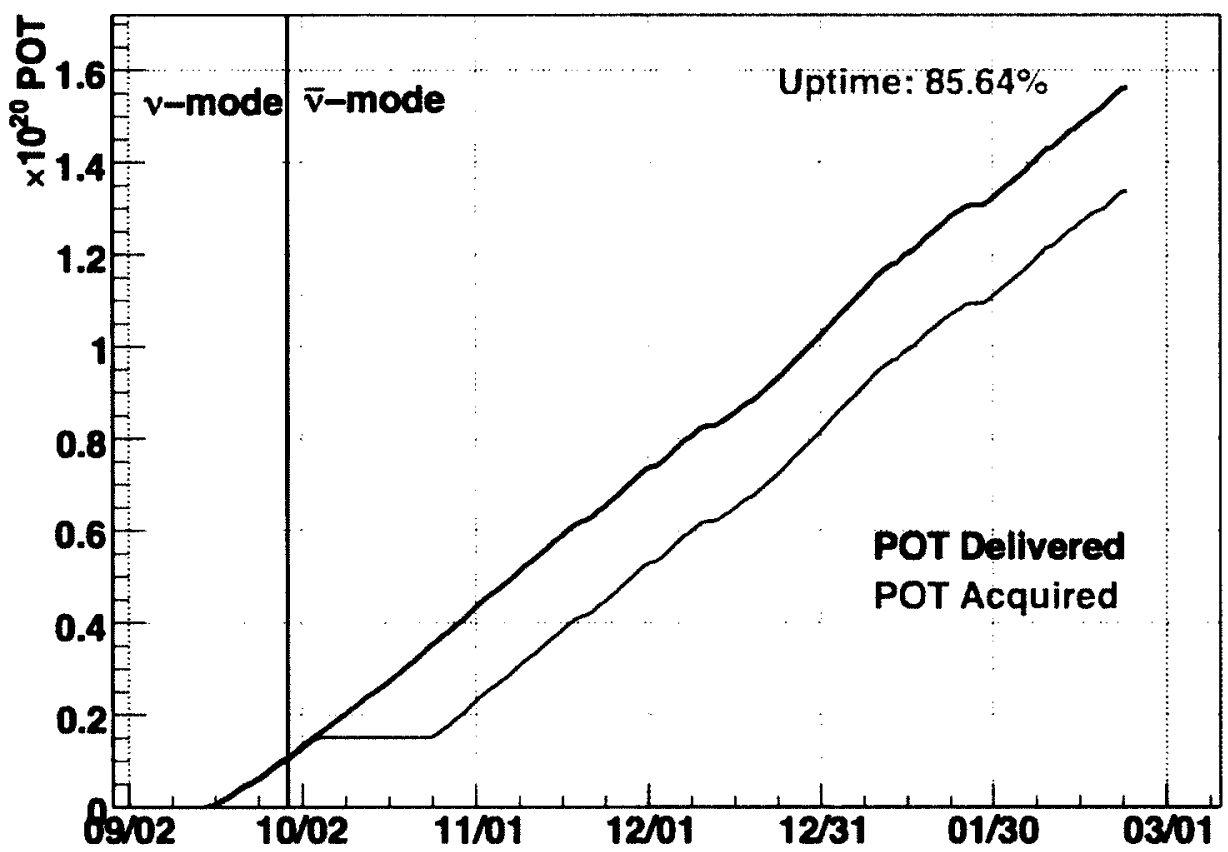

Figure 10.1: The ArgoNeuT physics run in terms of delivered and acquired protons on target (POT) as a function of time. The two week stop in operation was due to a failure of a commercial component of the cooling system. 


\section{Detector Performance}

In order to characterize and monitor the main parameters of the ArgoNeuT detector a large sample of through-going muons was used. ArgoNeuT's automated software, LArSoft described above, was employed to characterize the through-going tracks and the hits associated with them.

\subsection{Electron drift velocity}

The velocity of free electrons in liquid argon is of high importance as it is used to calculate the drift coordinate for the 3-dimensional tracks. The drift velocity depends on both the temperature of the liquid and the strength of the applied electric field. The field dependance of the drift velocity $\left(v_{d}\right)$ can be expressed as $v_{d}=\mu E_{d}$ where $E_{d}=481 \mathrm{~V} / \mathrm{cm}$ throughout the TPC drift region and $\mu$ is the electron mobility. The temperature of the liquid is $88.4 \pm 0.1 \mathrm{~K}$ as determined by the gas pressure above the liquid controlled and actively adjusted around the set point value of $2.0 \pm 0.2 \mathrm{psig}$. The temperature probes inside the cryostat confirm this value as well.

Direct measurement of the drift velocity can provide important information on the operation of the LArTPC. There are two classes of crossing muons that can be used for the determination of the drift velocity. First, the ionization tracks crossing the entire drift distance can be used for this measurement. The drift time from the cathode to the anode wire plane can be obtained by taking the time difference between the two hits: one detecting the entry point and the other exit point of the track. Then drift velocity is simply calculated as the drift distance to time ratio. However, these tracks must be at large incident angle with respect to the beam in order to cross the entire drift distance and are unlikely in 
ArgoNeuT. The second class of crossing muons suitable for this measurement are throughgoing tracks that cross one of the two planes, either cathode or the anode wire plane, by measuring their entry or exit time. The shield plane, delimiting the TPC drift volume opposite to the cathode, is not instrumented for signal read-out. Thus, the Induction plane is used for drift time measurement. Drift distance includes the detector drift length (470 $\mathrm{mm}$ ) and the gap between the Shield and Induction plane, which is at a higher value of electric field. A correction for the different field in the gap will be taken into account in addition to a correction due to thermal contraction of the TPC frame from room to LAr temperature in the drift direction. The distribution of the reconstructed hit times in the Induction plane from a sample of neutrino induced through-going muon tracks that cross either the cathode or the anode plane is shown in Figure 11.1.

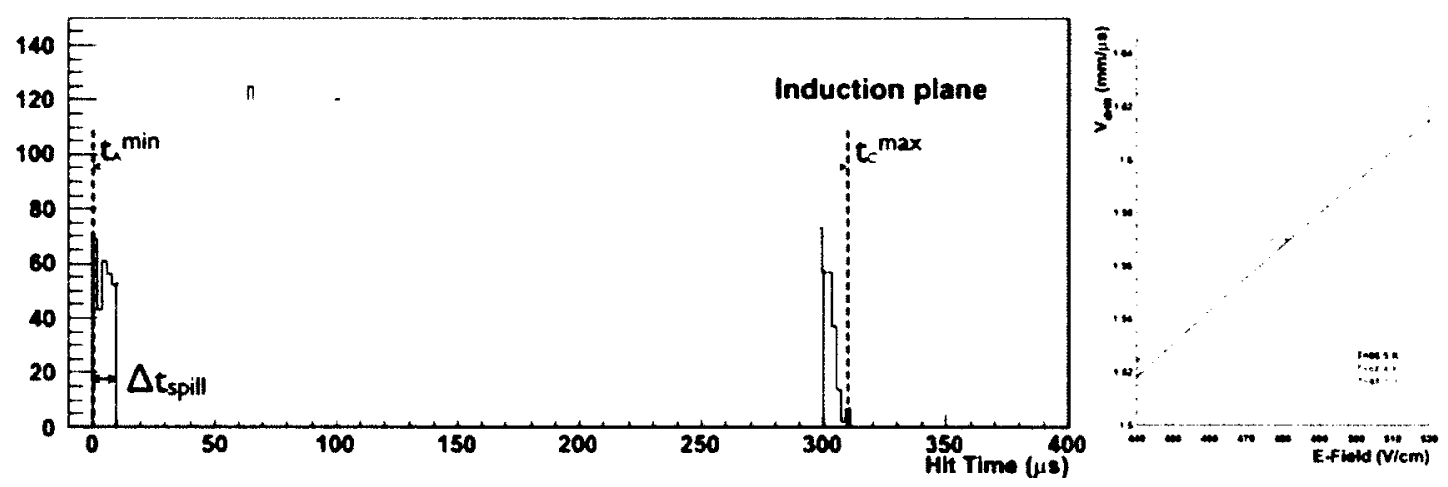

Figure 11.1: The left plot shows hit time distribution from the Induction plane for neutrino induced through-going muon tracks. The sample includes tracks crossing either the cathode or the Induction plane of the TPC. These tracks provide the hits at the edges of the distribution and thus determine the drift time measurement. The plot on the right shows the drift velocity measurement in ArgoNeuT, and associated systematic uncertainties, and comparison with expectation values from [115] as a function of the electric field and for different LAr temperatures corresponding to the range of variation of the current LAr temperature during operations.

Neutrino interactions are randomly distributed in time within the NuMI spill duration $\left(\Delta t_{\text {spill }}=9.7 \mu \mathrm{s}\right)$, thus the absolute hit time corresponding to a single plane crossing is also distributed over this time interval. The earliest neutrino interactions, at the beginning of the spill, will produce tracks with the minimum value of the absolute hit time $\left(t_{I}^{\text {min }}\right)$ when crossing the Induction plane, while the latest neutrinos in the spill produce tracks with maximum hit time $\left(t_{C}^{\max }\right)$ when crossing the cathode. The drift time $t_{d}$ (from cathode to Induction plane) is determined from the hit time distribution of the through-going tracks 
according to Equation 11.1:

$$
t_{d}=t_{C}^{\max }-t_{I}^{\min }-\Delta t_{\text {spill }}=300.5 \mu s
$$

by obtaining the values from Figure 11.1. Finally, the electron drift velocity is calculated according to Equation 11.2:

$$
v_{d}=\frac{l_{d}+l_{g} / r_{T 1}-\Delta l}{t_{d}}=1.57 \pm 0.02 \mathrm{~mm} / \mu \mathrm{s}
$$

The first correction to the drift length is accounting for the different electric field in the gap between the Shield and Induction plane, and the second one for the thermal contraction. The measured value is in very good agreement with expectations based on a common parametrization [115] of the electron drift velocity in liquid argon as a function of electric field strength, in the actual range of LAr temperature during the run, as reported in Figure 11.1 (right).

\subsection{Electron lifetime}

The key ingredient in the calorimetric reconstruction of ionization tracks is electron lifetime. Its accurate evaluation is also an indicator of the level of chemical purity of the liquid. The electron lifetime allows to account for the free electron loss during the drift time due to electro-negative impurities. The level of impurities depends on the filters removal efficiency and saturation level during operation in the recirculation/recondensation loop and thus can vary with time. ArgoNeuT measured the electron lifetime per DAQ-run which corresponds to about 21 hours. For the fully automated off-line procedure for the lifetime extraction a large sample of through-going muon tracks is used. They are suitable for this measurement as they have a narrow and known energy spectrum, almost constant directionality and $\mathrm{dE} / \mathrm{dx}$. The accelerator beam-timing signal is used as the neutrino event trigger for these muons. Selected tracks must span at least 120 wires in both Induction and Collection planes (for a detailed analysis of the through-going muon sample see [84]). The peak amplitude of every hit is then plotted as a function of drift time, separately for each plane. An example of this plot is shown in Figure 11.2. The drift time is clearly seen together with pre- and post-sampling regions. The set hit threshold is also clearly visible. The most prominent feature of the plot is the downward trend indicative of the non-infinite electron lifetime. 


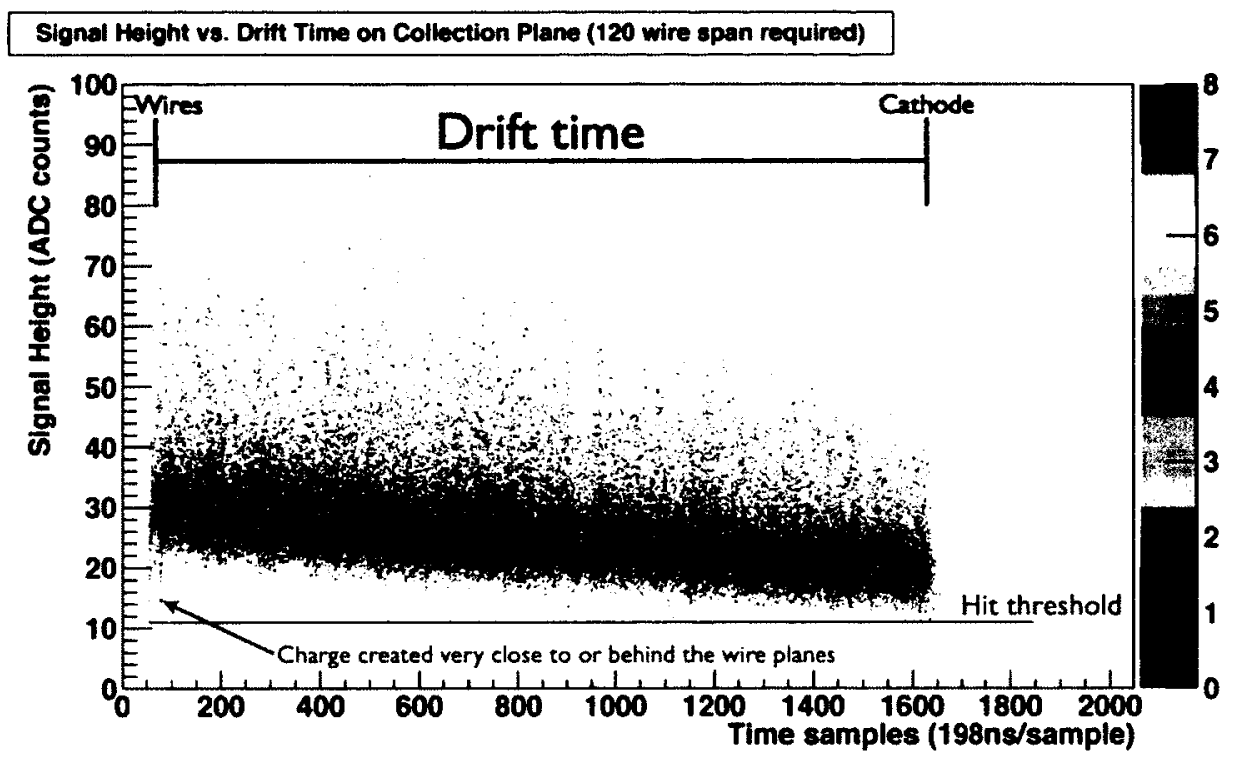

Figure 11.2: A 2-dimensional scatter plot of signal height as a function of drift time showing every hit associated with a long track on the collection plane in neutrino mode running.

Each of the 2D scatter plot is broken up into eight-samples-wide time slices. For each time slice, amplitudes of the hits are distributed in a $1 \mathrm{D}$ histogram. The value of time slice width was chosen to minimize the smearing effects of a finite electron lifetime and to have reasonable statistics. Each of the $1 \mathrm{D}$ histograms are fitted with a convoluted LandauGaussian distribution. The Gaussian distribution accounts for fluctuations in the detected charge due to electronic noise, differences in track pitch length, electron diffusion and energy spectrum of the tracks while the Landau distribution accounts for the features of the energy loss by ionization. Among the parameters to each fit there is the most probable value. Next, a separate fit is employed for each of the 190 time slices of the total drift time. A plot of the most probable value as a function of drift time for the Collection plane is shown in Figure 11.3. To extract the electron lifetime an exponential function fit is made for each plane. Finally, the two independent measurement are combined to obtain electron lifetime for DAQ-run.

The electron lifetime is minimally dependent on the time slice width, varying less than one percent after defining widths of $4,8,16$, and 32 time samples. It should be noted that the measurement could also be done by using the integrated area of the wire signal ADC counts instead of peak height and is found to give similar results. The lifetime as a function of DAQ run number for the entire ArgoNeuT data taking period is shown in Figure 11.4. 


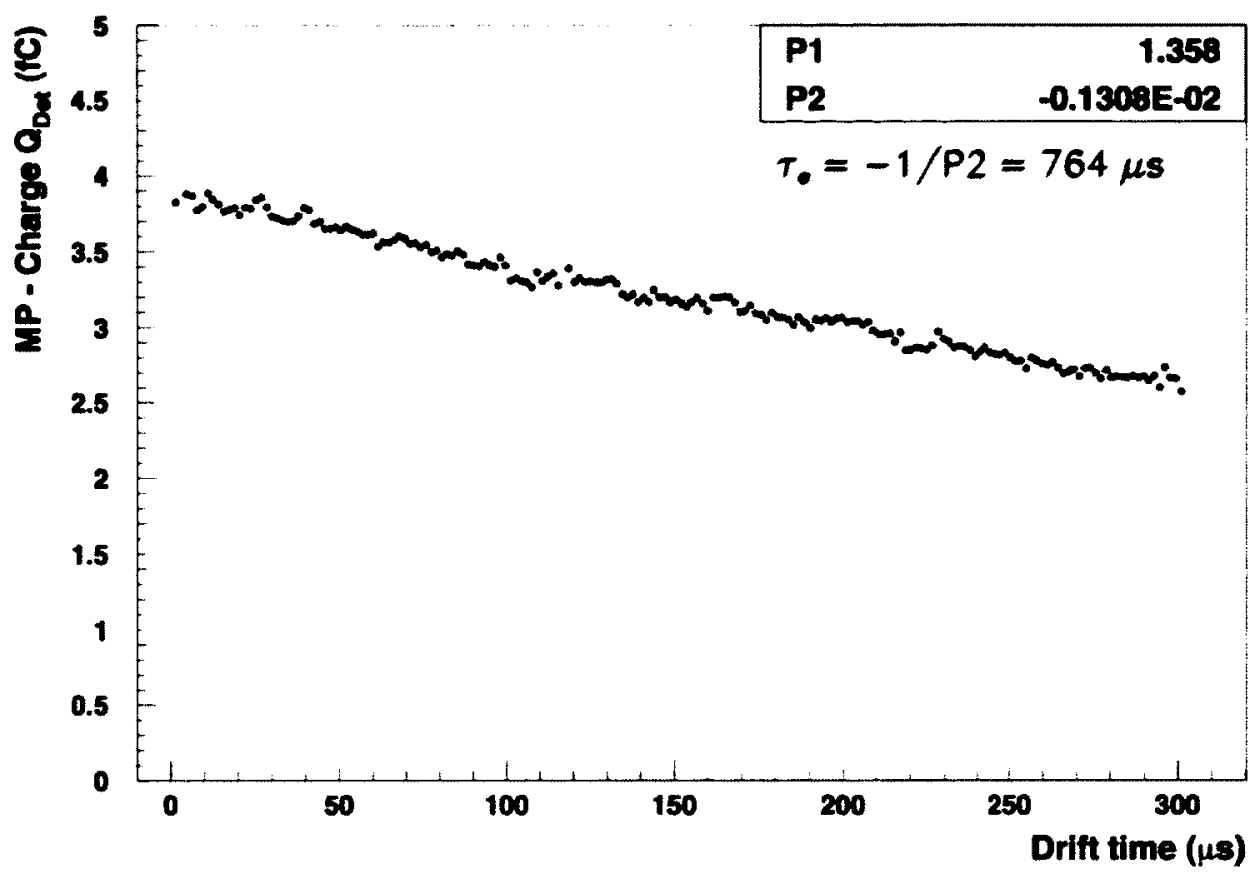

Figure 11.3: The e-lifetime extraction from the Collection plane with an exponential fit to the data. The fit gives an e-lifetime of $764 \pm 3_{\text {stat }} \mu$ s (statistical error only) for a specific DAQ-run in neutrino mode.

The total concentration of electro-negative impurities in liquid argon is usually reported in terms of the Oxygen equivalent $\left[\mathrm{O}_{2}\right]$ concentration which is expressed in ppb units (partsper-billion). The impurity concentration can be inferred from the measurement of electron lifetime and is shown for the entire ArgoNeuT run in Figure 11.5. A level of $1 \mathrm{ppb}$ is the sustainable impurity concentration given ArgoNeuT's maximum drift time of $300 \mu \mathrm{s}$. The higher level is obtained by a continuous operation of the purification system. 


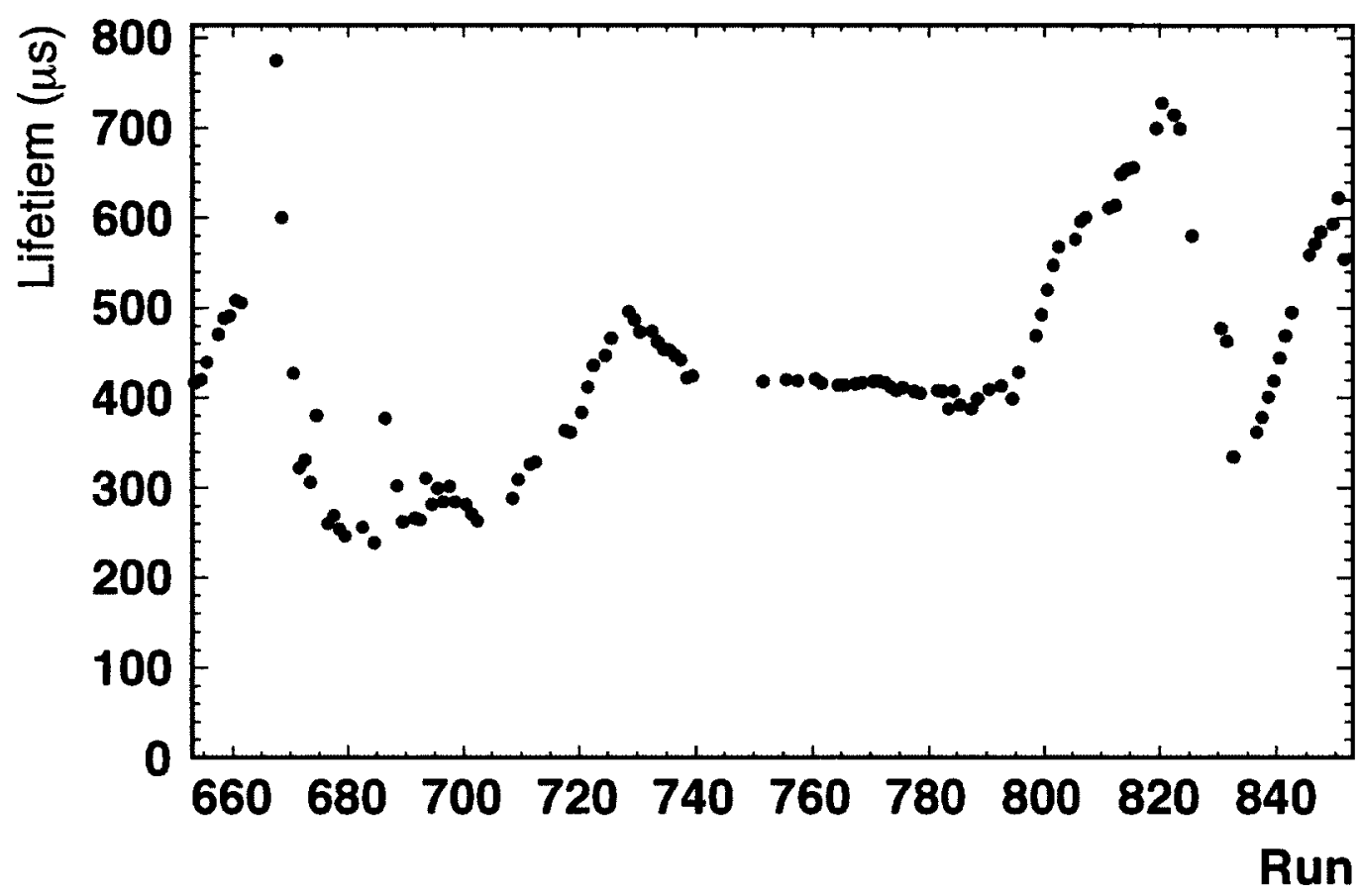

Figure 11.4: The e-lifetime as a function of run number for the entire ArgoNeuT data taking period.

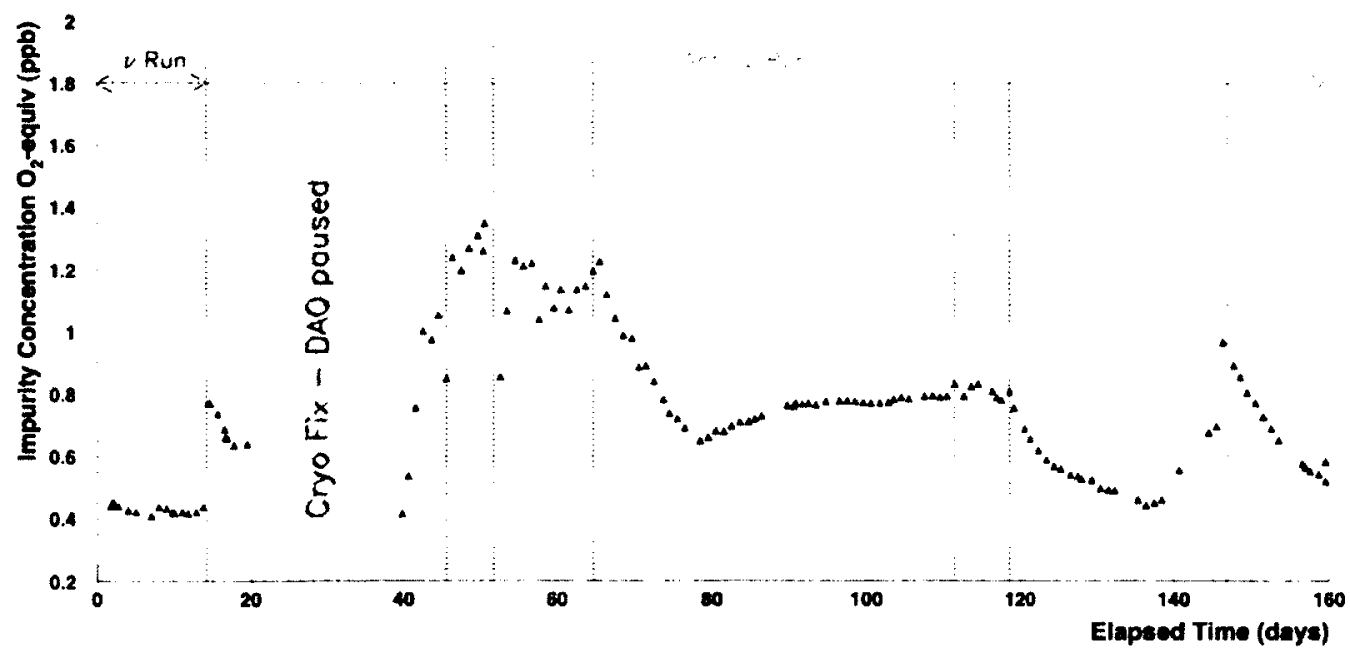

Figure 11.5: The $\mathrm{O}_{2}$ equivalent impurity concentration in LAr (ppb) extracted from each DAQ-run as a function of elapsed time (days) since the start of the ArgoNeuT physics run (about 160 days, beam in neutrino and antineutrino- mode). Vertical lines and band indicate hardware interventions on the purification/recirculation system: [red dotted] Filter exchange, [green dotted] GAr purge, [gray shaded] replacement of a cryocooler component - recirculation was halted during this period (about two weeks) and DAQ paused. 


\section{PART IV}

ArgoNeuT Simulation and Reconstruction of Events 


\section{Simulation}

The ArgoNeuT Monte Carlo plays a crucial role for reconstruction algorithm tuning, selection of proper cuts and comparisons with data. The simulation is also important for understanding of reconstruction efficiency and resolution for variety of neutrino interactions and analysis. The ArgoNeuT simulation consists of neutrino event generation, propagation of particles through a detector model, a model of drift and diffusion of the ionization electrons, creation of the wire signal and model of the electronics response to these signals. Simulated particle trajectories are reconstructed in the same way as for the data. Furthermore, the simulation includes propagation of particles that leave ArgoNeuT into MINOS Near Detector.

\subsection{GENIE Event Generator}

The ArgoNeuT experiment uses GENIE neutrino event generator [89] written for the experimental neutrino physics community. GENIE is the main software used for particle generation. It has been adopted by majority of neutrino experiments and is an important tool for the accelerator neutrino program. The current emphasis of the GENIE software is in the few $\mathrm{GeV}$ energy range, which is a boundary region between the non-perturbative and perturbative regimes relevant for accelerator-made beams. The present version provides modeling of neutrino interactions from $100 \mathrm{MeV}$ to a few hundred $\mathrm{GeV}$. The long term goal is to extend its validity to all nuclear targets and neutrino flavors from $\sim 1 \mathrm{MeV}$ to $\sim 1$ $\mathrm{PeV}$. GENIE is based on ROOT and developed entirely in $\mathrm{C}++$. Nothing has been changed in GENIE except specifying argon as the interaction medium at run time for ArgoNeuT.

Simulating neutrino interactions is a big challenge. Neutrino event generator developers 
need to piece together models with different range of validity for nuclear physics, hadronization models, and cross sections, among others. The most fundamental problem is lack of data as stated by the authors of neutrino generators. Most existing simulations have been tuned to data from bubble chamber experiments from 70's and 80's. The lack of available data or its limited size leads to substantial uncertainties in the simulations.

\subsubsection{GENIE Cross Section Model}

Flux and total cross section is used to determine the neutrino energies. The cross sections for specific processes are used to determine the interaction type. The event kinematics are extracted from the differential distributions for the interaction model.

\section{Quasi-Elastic Scattering}

The Quasi-Elastic Scattering (CCQE) is shown in Figure 12.1 and has only an outgoing proton and muon and thus is one of the easiest topologies. It constitutes the biggest fraction of $\mu+N p$ topological events presented in this thesis. GENIE models this interaction via an implementation of the Llewellyn-Smith model [102] where the hadronic weak current is expressed in terms of the most general Lorentz-invariant form factors. The used parametrization model of these electromagnetic form factors is BBBA2005 [103], while GENIE has many others available. The only free parameter is the axial vector mass $M_{A}$ with the set default value of $0.99 \mathrm{GeV} / c^{2}$.

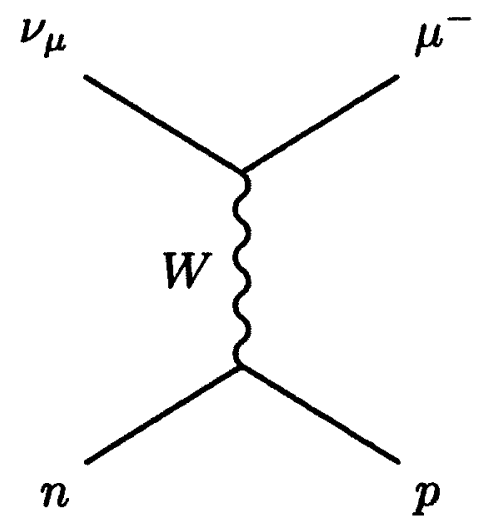

Figure 12.1: A Charged Current Quasi-Elastic (CCQE) interaction 


\section{Elastic Neutral Current Scattering}

Neutral Current interaction is shown in Figure 12.2. GENIE implements this process according to the model described by Ahrens et al. [104] with one adjustable parameter. It will be interesting to study this interaction in future LArTPCs as ArgoNeuT is too small to tag the neutrons. Another motivation for exploring this interaction is a clear deficit of available data for this process.

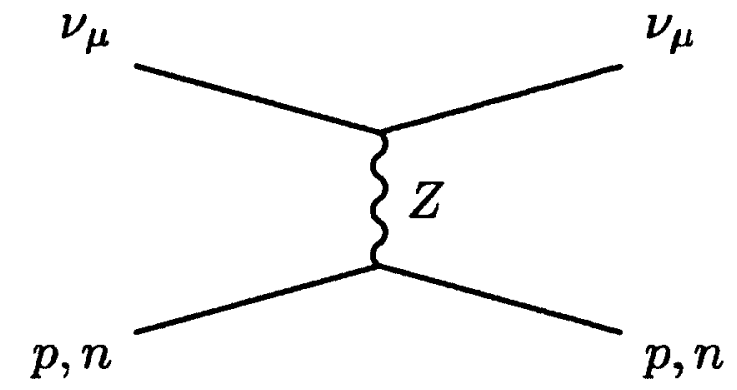

Figure 12.2: A neutral current elastic interaction

\section{Coherent Neutrino-Nucleus Scattering}

There is coherent scattering resulting in both charged current and neutral current channels as shown in Figure 12.3. The pions are produced in the forward direction with respect to the neutrino. GENIE models the interactions via the Rein-Sehgal model [105]. The coherence condition requires a small momentum transfer to the target nucleus. It is a low- $Q^{2}$ process in which the Rein-Sehgal model starting from PCAC form and calculates the pion-nucleus cross sections from the data on total and inelastic pion scattering from protons and deuterium [106]. GENIE uses the modified version of PCAC formula [107] that differs from the previous one by the lepton mass term.

\section{Deep Inelastic Scattering}

Deep Inelastic Scattering shown in figure 12.4 is calculated in an effective leading order model using the modifications suggested by Bodek and Yang [108]. A new scaling variable and modifications to the low $Q^{2}$ parton distributions are used to account for target mass corrections. The cross sections are computed at partonic level. Nuclear effects are included by using Whitlow parametrization [109]. 

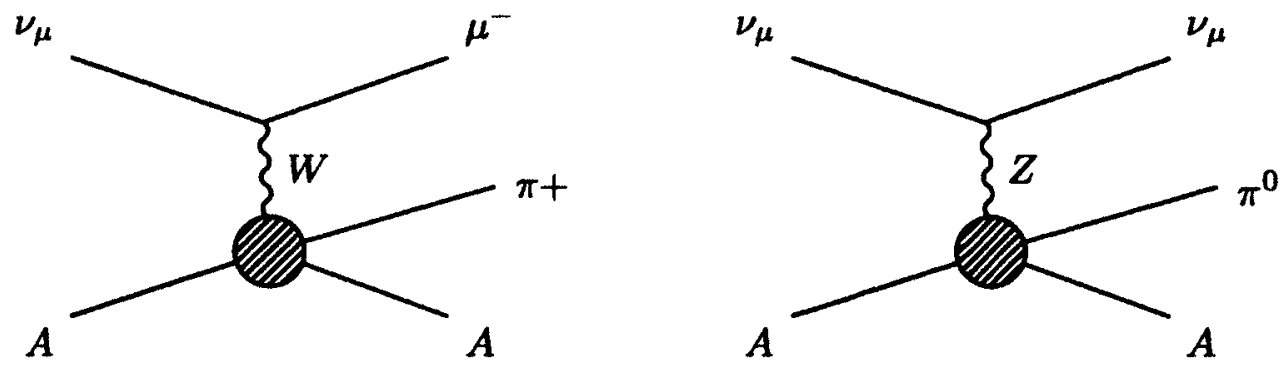

Figure 12.3: A charged current coherent (left) and neutral (right) interaction
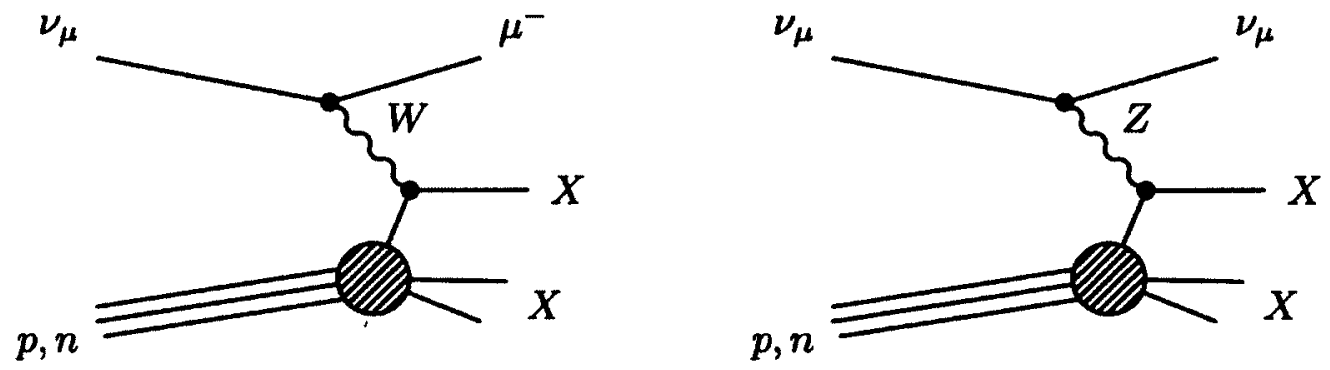

Figure 12.4: A charged current DIS (left) and neutral DIS (right) interaction

\section{Single Pion Resonant}

This channel, shown in Figure 12.5, is especially important for $\mu+N p$ analysis in the case of pions that underwent FSI and are not present in the final state. In this form the interaction satisfies the topological appearance of $\mu+N p$ events. The production of these interactions is modeled according to Rein-Sehgal model [110]. The model uses FeynmanKislinger-Ravndal [111], which "gives wave functions for the resonances as excited states of a 3-quark system in a relativistic harmonic oscillator potential with spin-flavor symmetry." GENIE implements 16 out of 18 resonances from the latest PDG baryon tables.

\subsubsection{Nuclear Physics Model in GENIE}

Nuclear physics plays a crucial role in neutrino scattering simulations at few-GeV energies in GENIE. There is an energy dependence determination of a particle in the nucleus with which the neutrino interacts. At lower energies neutrinos interact with neutrons and protons. The neutrino tends to strike a single nucleon, which is affected by the nuclear medium around it. At high energies, greater than $10 \mathrm{GeV}$, neutrinos interact with a single quark inside a neutron or proton. 

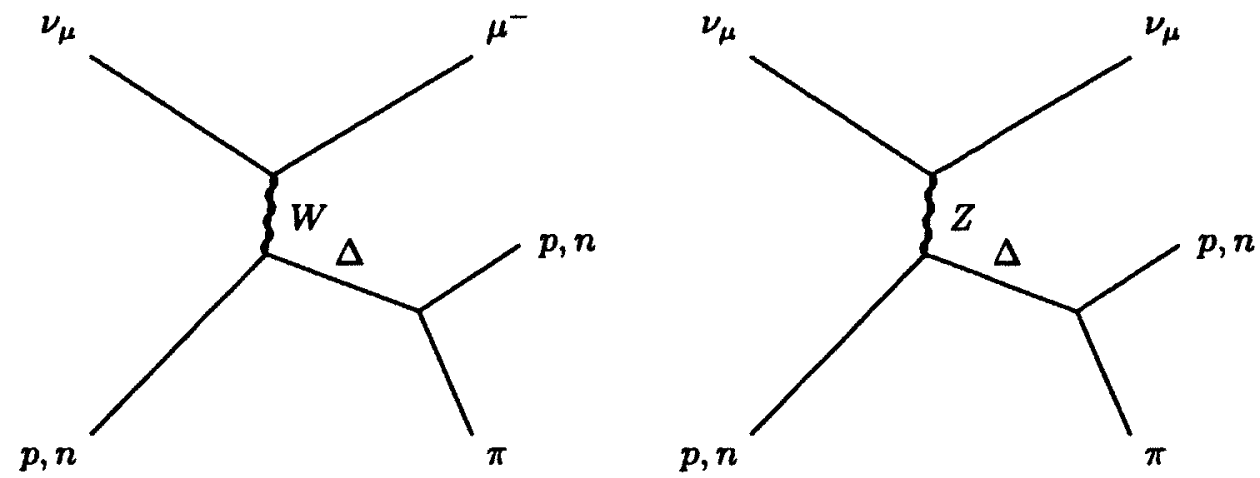

Figure 12.5: A charged current single pion resonat (left) and neutral single pion resonant (right) interaction

One of the largest differences among models of the neutrino-nucleus interaction is the treatment of the Final State Interactions (FSI). It involves many aspects of nuclear and strong interactions. However, there are many effects to include and there is a substantial disagreement about the best techniques to implement.

The relativistic Fermi gas (RFG) nuclear model is used for all processes. GENIE uses the version of Bodek and Ritchie which has been modified to incorporate short range nucleonnucleon correlations [90], applicable across a broad range of target atoms and neutrino energies. Tests of the RFG model come from electron scattering experiments [91]. At lower energies impulse approximation works well, at higher energies the nuclear model is far more complicated. A momentum and average binding energy is given to the struck nucleon by the nuclear medium. The model can be used for all nuclei. In the case of argon, 2-parameter Woods-Saxon density function is used [92]. Scattering kinematics for free nucleons are different than bound nucleons. Pauli blocking is implemented for quasielastic and elastic scattering. Cascade Monte Carlo is used for nuclear reinteractions of produced hadrons. The struck nucleus, left in an excited state can de-excite by emitting nuclear fission fragments, photons and nucleons. The present version of GENIE does not contain de-excitation photon emission for argon.

Hadrons produced in the nuclear environment may rescatter as they exit the nucleus. These reinteractions can alter the observable distributions, which is why the presented here analysis is topological in nature. It is also well known that hadrons produced in the nuclear environment do not immediately reinteract with their full cross section. Quarks can propagate through the nucleus with very reduced interaction probability. GENIE models 
it as a "free step" at the beginning of the intranuclear cascade during which interactions are prohibited. The used model is SKAT [93] with a formation time of $0.342 \mathrm{fm} / \mathrm{c}$.

A package INTRANUKE is employed in GENIE for intranuclear hadron transport, which is an intranuclear cascade simulation. It basically simulates rescattering of nucleons and pions in the nucleus. Hadrons produced in a nucleus have a typical mean free path of few femtometers. The hadrons produced directly by neutrino often undergo Final State Interactions (FSI). These can drastically change the final state topology. As an example, a genuine Charged Current Quasi-Elastic (CCQE) neutrino interaction that has one proton emitted can end up with a final state of 3 protons in addition to multiple neutrons and photons. Another possibility, relevant for $\mu+N p$ analysis, is a pion production where the pion is absorbed. Such an event will look like a CCQE event. In order to understand this a high quality Monte Carlo is needed.

There are variety of models but a big problem is the lack of available data for cross check. The models most important for neutrinos are intranuclear cascade(INC) $[94,95,96]$. These models are semi-classical where the hadron sees a nucleus consisting of isolated nucleons (neutrons and protons), which have Fermi motion and binding energy. The incident particle interacts in a series of encounters with single nucleons called a cascade. All interactions are ruled by the cross section for the free process and probability of interaction is governed by a mean free path. The probability of interaction is given by density of nucleons and free cross section as shown in Equation 12.1

$$
\lambda(E, r)=\frac{1}{\rho(r) * \sigma_{h N, t o t}}
$$

A typical nucleus is approximately a sphere of radius $\mathrm{R} \propto A^{1 / 3}$. In the case of ArgoNeuT, the charge density is described by the Woods-Saxon shape as shown in Equation 12.2.

$$
\rho(r)=N_{0} \frac{1}{1+e^{(r-c) / z}}
$$

where $z$ describes the width of the surface of a nucleus and $c$ the size. The INC models are considered the most significant as they can more easily than others describe a wide range of available data.

The FSI model implemented in GENIE and used by ArgoNeuT is an empirical, datadriven model called hA. A total cross section for each possible nuclear process is used for pions and nucleons as a function of energy. At low energies there is sufficient data 
$[97,98,99,100,101]$ but at higher energies ( greater than $300 \mathrm{MeV}$ ) only a few data points are available. The calculations are not in agreement with the existing total cross section data and thus were normalized to the data at low energies. This model also takes care of proton and neutron rescattering. First application was employed by MINOS and thus there was much emphasis on iron, for other targets cross sections are obtained by scaling by $A^{2 / 3}$.

Some common terminology used for ways of interactions of hadrons with nuclei are: elastic scattering, charge exchange, absorption, spallation and pion production. When the final state nucleus is in its ground state and the hadron has the same charge as the beam particle the interaction is referred to as elastic scattering. In case of inelastic scattering the nucleus is either in the ground state or broken apart. The nucleus decays to a ground state and emits a photon at low excitation energies. At higher excitation energies multiple nucleons can be emitted and FSI can increase their number. In the scenario of a different charge in the final state in comparison to the initial, but the same particle type, the interaction is called 'charge exchange'. A common example is $\pi^{-} p \rightarrow \pi^{0} n$. When interaction is initiated by a pion but no pions are present in the final state the process is referred to as 'absorption'. It is one of the most important backgrounds for Charged Current Quasi-Elastic analysis. For incident nucleons, a multiple of them present in the final state is called 'spallation'. In case a pion is produced in the final state, while no pions are present in the initial state, the process is called 'pion production'. If the initial state contains a pion, the final state must have an additional pion in the final state for the interaction to be also called pion production. A schematic showing a FSI interaction and pion absorption as seen in an INC model is shown in Figure 12.6. It should be obvious that the interactions can be a mix of the mentioned processes and disentangling them is a real challenge.
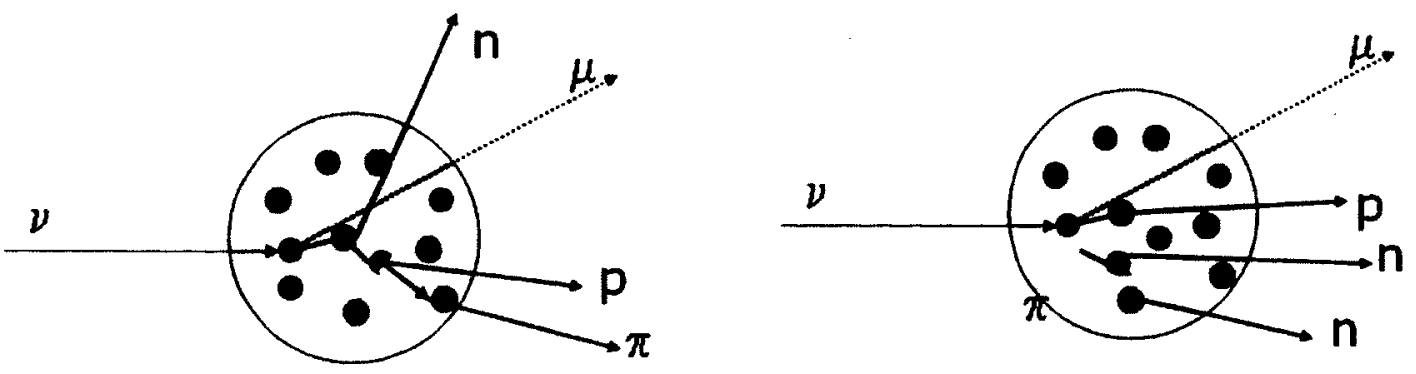

Figure 12.6: Schematic diagram for reaction involving FSI process (left) and reaction of the pion absorption (right). 


\subsubsection{GENIE Output}

The output of the GENIE simulation is a 'particle'. This unit describes particles in initial, intermediate, and finals state. Each particle is given a status code signifying its position in the time ordered simulation of the event. Particles can be marked as being in one of the following states:

- Initial State, usually the incoming neutrino and the nuclear target

- Nucleon target, refers to the struck nucleon within the nuclear target

- Intermediate State, refers to fragmentation intermediates or remnant nucleus

- Hadron in the Nucleus, refers to a particle from the primary interaction vertex before any re-interactions

- Decayed State, refers to unstable particles that have been decayed

- Stable Final State, refers to long-lived particles coming out from the nuclear target

\subsection{ArgoneuT Detector Geometry and Signal Simulation}

All particles produced by GENIE in the final state are propagated through the ArgoneuT detector parts with the use of the Geant4 software. The detector parts including the argon volume, the TPC, inner and outer cryostats, containment vessel, and first MINOS plane are created with the help of the Geometry Description Markup Language (GDML). Then they are read-out and simulated with the Geant4 software. Figure 12.7 and Figure 12.8 show the simulation of ArgoNeuT detector with the detailed model for the TPC, inner cryostat, outer cryostat, the containment vessel and MINOS first plane. The particles are stepped through the simulation automatically, once they reach kinetic energy greater than $100 \mathrm{keV}$ they become recorded. The ionization of the electron clouds is deposited into three dimensional voxels, which are pixels in three dimensions. The next step is the simulation of the drift of the ionization products to the wire planes. 

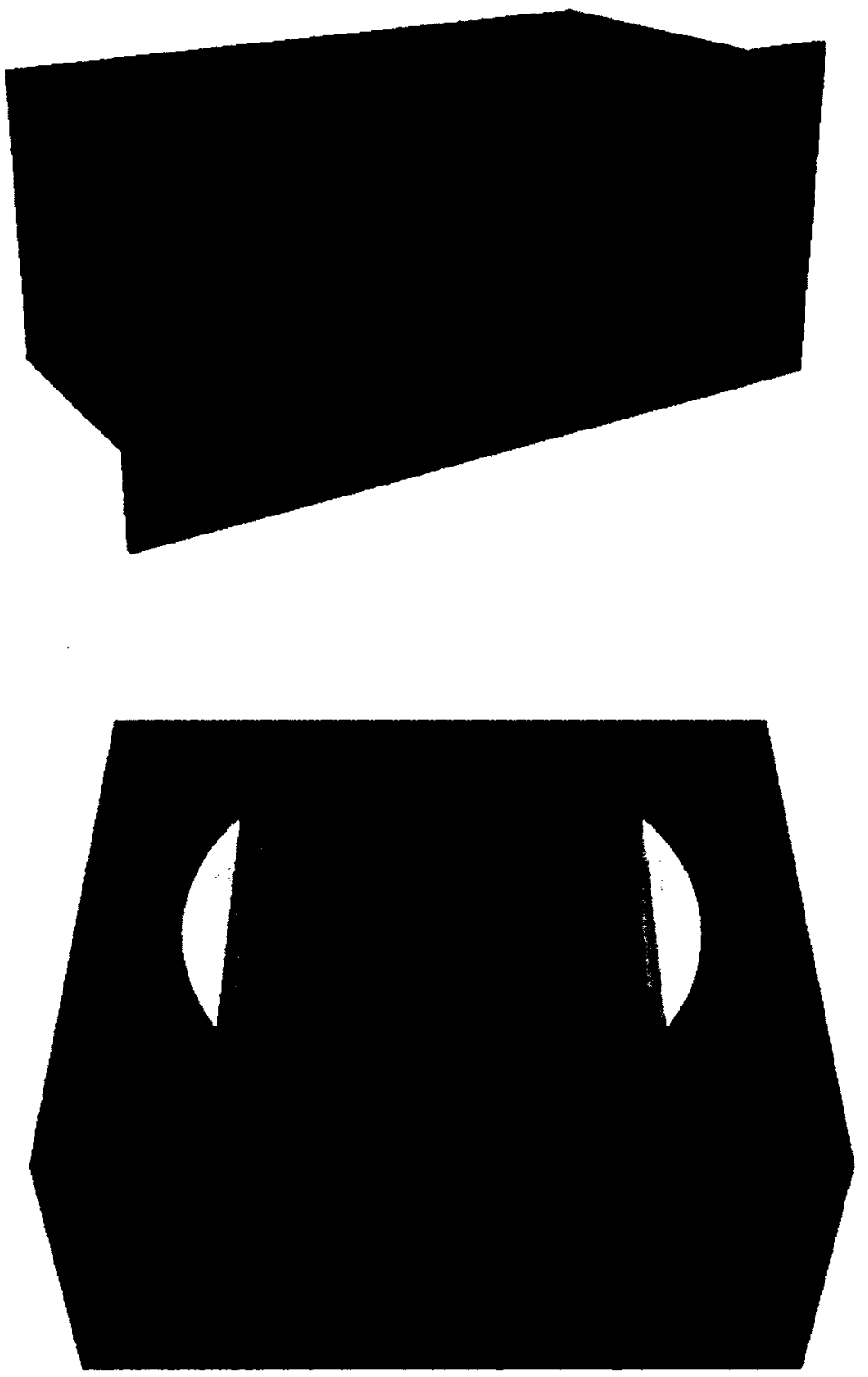

Figure 12.7: The ArgoNeuT TPC in Geant4 with the wire planes in the foreground (top). A few wires (in white) are shown to see their relative angles to one another. The cross section of the whole ArgoNeuT detector is seen in the bottom plot. Starting from the middle and going outwards, the TPC, the inner and outer cryostats are seen. The detector sits in an orange containment vessel. 


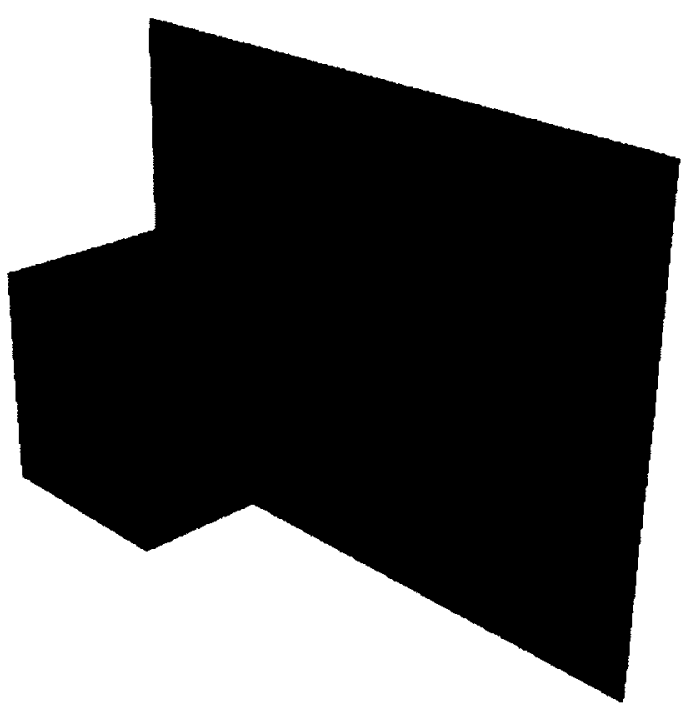

Figure 12.8: The simulation of the ArgoNeuT detector together with the first MINOS plane.

Once each charged particle deposits its energy in voxels, the electron clouds are drifted towards the readout planes. The track orientation and energy deposition are then used to determine the $\mathrm{dE} / \mathrm{dx}$. Longitudinal and transverse diffusion constants are set for the drift of the electrons to wire planes. The diffusion, of course, depends on the drift time of the electron clouds. The recombination effect is also simulated. Finally, the wires are assigned with the number of electrons that reached them after accounting for the diffusion and recombination. The electron clouds are turned into charge and associated with time ticks. Response shapes on Induction and Collection planes are convoluted with the charge to obtain raw digit ADC counts. To complete the electron drift and wire simulation a random noise is added to the wires. The dead or noisy wires that were found in the detector are given zero $\mathrm{ADC}$ counts for all time samples in the simulation and data.

\subsection{Propagation into MINOS Near Detector}

Muons in the MINOS Near Detector from upstream or "rock" neutrino interactions are captured with each NuMI trigger. They are employed in the simulation of beam-related background in the ArgoNeuT. In the ArgoNeuT simulation we take a random, properly normalized number of MINOS data muons and project them backward along their measured MINOS ND trajectory until they are just upstream of the front face of the ArgoNeuT 
cryostat. The data muons are then set aside, and simulated muons are then launched forward from this position with the MINOS measured momentum of the data muons. Any of these simulated muons which miss the ArgoNeuT cryostat by a wide margin (about 1 meter) are not propagated any further. Any which cross through the ArgoNeuT TPC are also not propagated further, because those have been identified and subtracted already by the hand-scanning process. The rest of the muons, however, are propagated through the ArgoNeuT simulated geometry and into the MINOS ND, where they are handed off to the MINOS ND simulation and reconstruction. These muons are then available for mismatching with the ArgoNeuT Charged Current muon or other charged, neutrino-related tracks. 


\section{LArSoft Software}

The Liquid Argon Software (LArSoft) is a common framework for all LAr TPC detectors. LArSoft contains simulation and reconstruction algorithms. Its general structure is well established but the individual algorithms are subject to constant improvement. The algorithms used for $\mu+N p$ analysis will be described here. It should be pointed out, that there exist other packages for different classes of events, for example showers, but they are beyond the scope of this analysis and they will not be explored here. The off-line reconstruction chain for $\mu+N p$ analysis consist of the following steps listed in the order of their application:

- Raw waveform treatment

- Hit identification

- 2-dimensional clustering

- 2-dimensional line reconstruction

- Vertex finding

- 2-dimensional, more refined clustering of short vertex tracks

- 3-dimensional track reconstruction

- Calorimetric reconstruction

- Matching 3-dimensional tracks with MINOS Near Detector

These algorithms will be described in the subsections below. 


\subsection{Raw Waveform Treatment}

The initial steps applied to the digitally recorded raw waveforms are noise filtering and electronics response deconvolution. In liquid argon TPC's the induction signal is bipolar while the collection plane features a unipolar current pulse which comes from charge converging on a wire as shown in Figure 13.1.

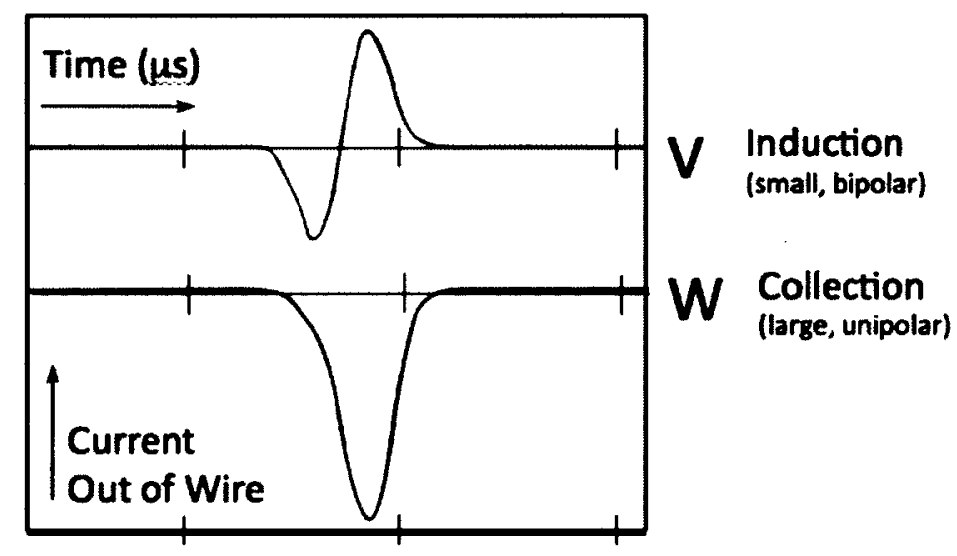

Figure 13.1: Current signals for the Induction and Collection wires as induced by a drifting charge.

The shape of the signal is preserved at the read-out electronic output but at the collection plane output signals are followed by a negative overshoot and exponential return to baseline. This inconvenience is caused by the capacitive coupling of ArgoNeuT's digitizer

inputs. In order to address these issues, a deconvolution scheme using the Fast Fourier Transform (FFT) algorithm [112] is employed. The bipolar induction signals are converted into unipolar shapes. Each Fast Fourier Transformed wire signal is divided by the result of an FFT transform of the Induction signal shape. The electronic field response and a filter cut out the low frequency noise. In the presence of noise, filtering the frequency space is a needed component of FFT deconvolution. In the absence of low-pass filtering, high frequency noise components are amplified above the signal. Due to the difference in the signal shaping for the two planes, this must be handled differently for each plane. In the Induction plane a smooth analytic function is employed that maintains low frequencies and attenuates high frequencies. In the Collection plane, Weiner filtering [112] is used. It effectively weights each frequency according to its power in the noise and signal power spectrum. The final FFT output waveforms are smooth and unipolar pulses from the wires in both planes. The pulses have similar rise and fall times and are symmetric. 
In order to identify the interesting regions of the waveform, a gaussian-shape approximation is used. These regions are called "hits" and will be discussed in the next section. An example of muon raw and de-convoluted waveform on a wire is shown in Figure 13.2. A bipolar signal on the Induction plane and a unipolar pulse on the Collection plane is seen. The deconvoluted waveform is shown (in blue) together with the identified hit (in red).
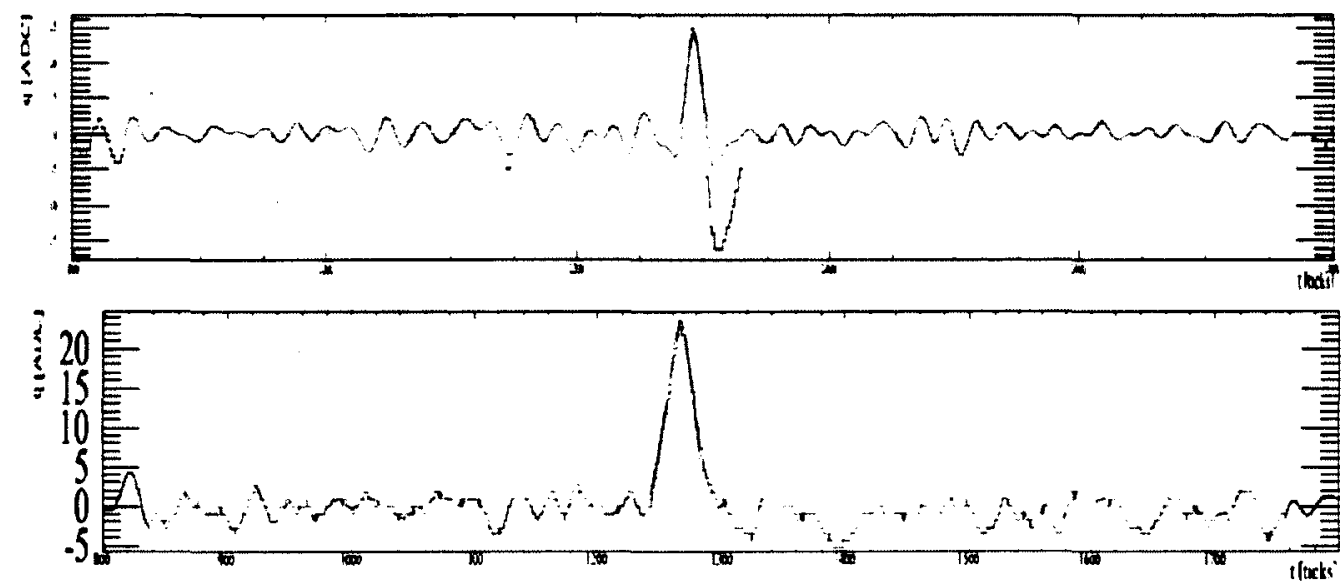

Figure 13.2: Wire raw waveform shown in black and deconvoluted waveform shown in blue for a crossing muon track in data, parallel to the wire planes.

\subsection{Hit Identification}

ArgoNeuT's hit finding is provided with the smooth and unipolar deconvoluted signals on each plane, as described above, and is performed on a wire-by-wire basis. The hit finding algorithm initiates a search for a local minima. If a minimum is found, the algorithm tries to find a local maximum by following the waveform. If the maximum is above a specified threshold the pulse is considered a hit. The program then scans to the next local minimum and identifies the region as a hit. If the local maximum is below threshold, it rejects that region and repeats the process again by scanning to the next local minimum. Once one or multiple hit regions are found, they are each fitted with a Gaussian function. The hit features are then derived from the fit to characterize it with time position, width and height. In case of consecutive multiple hit regions above threshold, a multiple hit finding procedure is performed to characterize individual hits. The build-in histogram fitting algorithm from the ROOT software package [112] is used. In order for the fit to converge, this algorithm requires seeding initial values to the parameters close to the correct ones. The initial half- 
width value is given as a parameter for the Induction and Collection planes separately. They are 6.0 and 7.8 ticks respectively as determined from typical single hit widths in the data. The local maximum positions are set as the initial positions and the height of the maximum as the initial signal amplitude. The peak height of multiple hits calls for a more complicated procedure due to added signal from nearby hits. A simple linear approximation is made to obtain the initial values for the individual hits. The measured height at one peak consists of its height in addition to the contributions from its neighbors. The measured height of peak i, $A_{i}^{*}$ is given by Equation 13.1:

$$
A_{i}^{*}=\sum A_{j} f\left(t_{j}-t_{i} ; w\right)
$$

where $f\left(t_{j}-t_{i} ; w\right)$ is the normalized model signal shape, w its width, $t_{i}$ the center position of peak $\mathrm{i}$, and $A_{i}$ the approximate signal height of hit $\mathrm{i}$ alone. The function $f$ determines how much the nearby hit or hits influenced the amplitude of the hit in question. The above equation can be considered as $\overrightarrow{A^{*}}=M \vec{A}$ where $\vec{A}^{*}$ is the observed hit amplitude and $\vec{A}$ the approximate one. $\mathrm{M}$ is formed by the values of the normalized model function for each hit evaluated at the point of the other hits. It is worth noting that this matrix is symmetric with diagonal entries being a unity, leaving less than half of the matrix to evaluate. This system gives a good approximation of the initial signal amplitudes.

Deconvoluted waveforms are scanned in order to find hits in each plane separately. Each hit is characterized by wire number, time tick (position), amplitude and width as determined from the fit. The hit start and end times are recorded as the center position minus and plus the width, respectively. To visually indicate low and high local ionization density, the ADC scale of the hit amplitude is converted into a color level scale. On this scale yellow is indicative of low ionization density and red the highest one as shown in Figure 13.3 for both Induction and Collection planes. The display shows raw and deconvoluted signals from three adjacent wires.

The results of the hit identification process are passed for each plane to subsequent reconstruction methods that cluster the hits together separately for each plane.

\subsection{2-dimensional Clustering}

Clustering means grouping of objects into meaningful subclasses. The problem is very challenging when the clusters are of different shape, size and density. The issue becomes even 


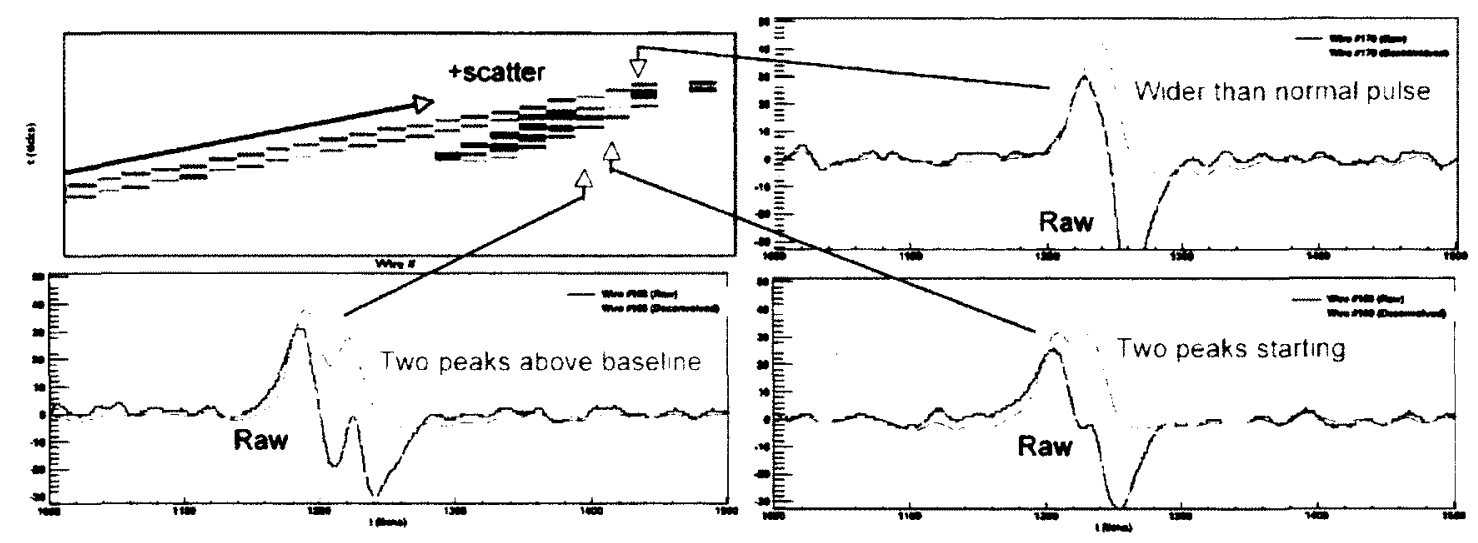

Figure 13.3: Induction plane view of two tracks (upper left) with wire views on three adjacent wires. The raw data is shown in black and the deconvoluted data in red.

bigger when data includes noise. ArgoNeuT collaboration has employed a well known in computer science algorithm that offers solution to the mentioned challenges and is efficient. Hit clustering is accomplished through a process based on "Density-Based Spatial Clustering of Applications with Noise" (DBSCAN) [112]. DBSCAN's idea of a cluster is based on "density reachability". A point $q$ is "directly density-reachable" from a point $p$ if it is within a given neighborhood and if $\mathrm{p}$ has sufficiently many points surrounding it so that both of these points can be considered a part of the same cluster. On the other hand, $q$ is called "density-reachable" from $\mathrm{p}$ if there is a sequence of points $p_{1}, \ldots, p_{n}$ with $p_{1}=p$ and $p_{n}=q$ where $p_{i+1}$ is directly density-reachable from $p_{i}$. There are two kinds of points in a cluster, points inside of the cluster called core points, and points on the border of the cluster called border points. Directly density-reachable is symmetric for pairs of core points. However, it is not symmetric if one core point and one border point are involved as depicted in Figure 13.4. Due to this asymmetry a definition of "density-connected" is needed. Two points $p$ and $q$ are said to be density-connected if there is a point o such that both $\mathrm{p}$ and $\mathrm{q}$ are density-reachable from $\mathrm{o}$. A schematic of the asymmetric case on some points is depicted in Figure 13.5. Density-connectivity is a symmetric relation and a cluster can be defined based on this notion. A cluster is defined as a set of density-connected points. Noise is defined simply as points that do not belong to any cluster. DBSCAN starts with an arbitrary point $\mathrm{p}$ and finds all the points that are density-reachable from $\mathrm{p}$ with respect to some defined neighborhood. If $\mathrm{p}$ is a core point a cluster is started. If $\mathrm{p}$ is a border point, DBSCAN visits the next point as no points are density-reachable from $\mathrm{p}$, and $\mathrm{p}$ is marked 
as noise. This point might be found later to fall within some other point's sufficiently sized neighborhood and hence be made part of a different cluster. This process iterates until the density-connected cluster is completely found. In the case of ArgoNeuT the points are hits passed to DBSCAN as 2-dimensional points with (wire, time) values. The neighborhood is defined by an elliptical region with the semi-major and semi-major axis as parameters $\epsilon_{1}$ and $\epsilon_{2}$. Some examples of DBSCAN at work on data events are shown in Figure 13.6 on line-like event and in Figure 13.7 on shower-like event.
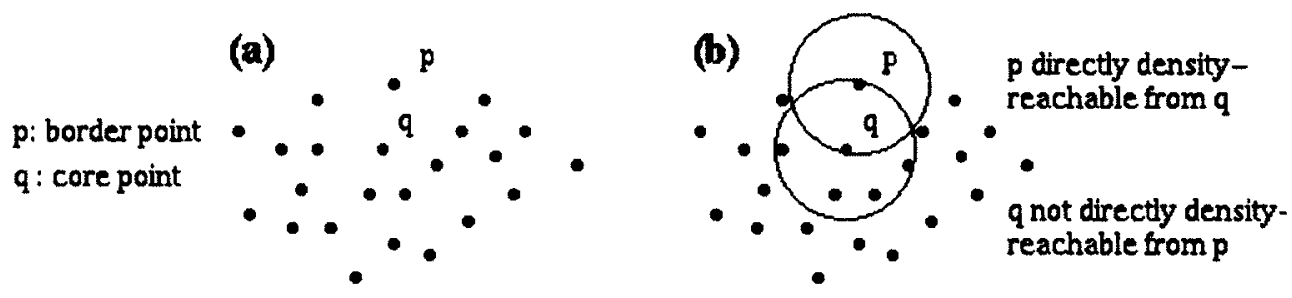

Figure 13.4: Core points and border points (adopted from Reference [112])

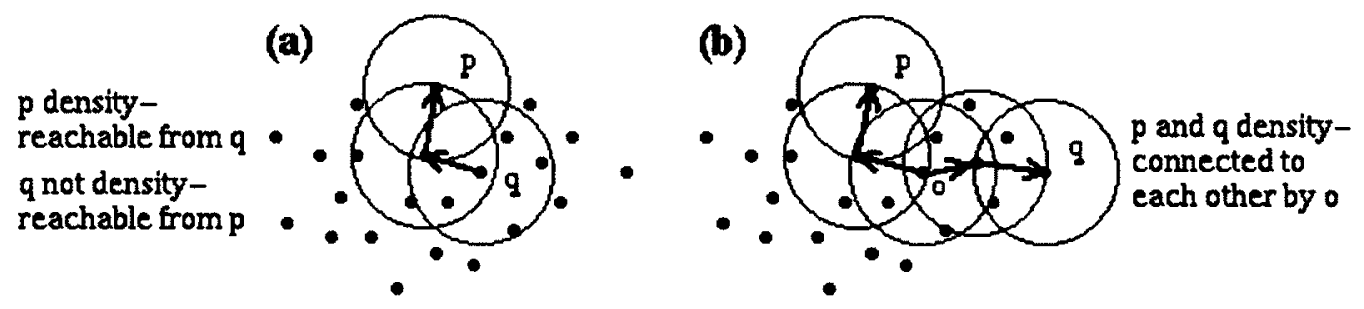

Figure 13.5: Density-reachability and density-connectivity (adopted from Reference [112])

\subsection{2-dimensional Line Reconstruction}

Most neutrino events in ArgoNeuT feature line-like track as the detector is not magnetized. Thus, a line recognition algorithm has been implemented to search for and form 2-dimensional clusters with a possibility of multiple lines per event. It should be noted that this task can be challenged by noisy and dead pixels, low- resolution, non-standard shapes and multiple scattering to name a few. A Hough transform [112] was implemented to identify all line-like objects. The Hough transform creates a parameter space, or accumulator, filled according to the hit locations. A line can be parametrized by $r=x * \cos (\theta)+y * \sin (\theta)$, which represents a curve in the $(r, \theta)$ plane unique to each $(x, y)$ point. If the curves corresponding to two points are overlaid, the $(r, \theta)$ crossing point represents the parameters of a 

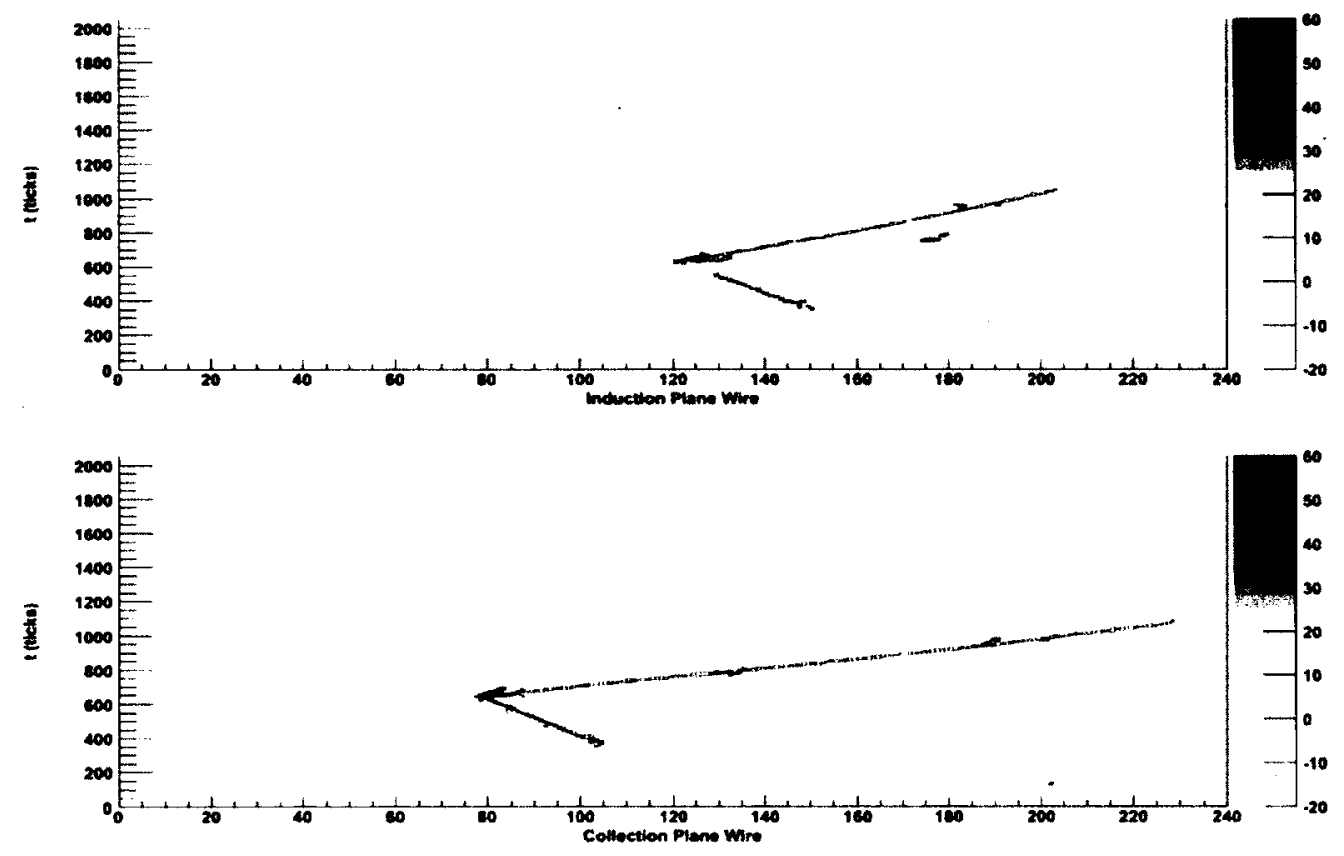

Figure 13.6: The DBSCAN clustering algorithm ran on a neutrino event. The display shows colored hits on the induction and collection plane, each color corresponds to a unique cluster formed.

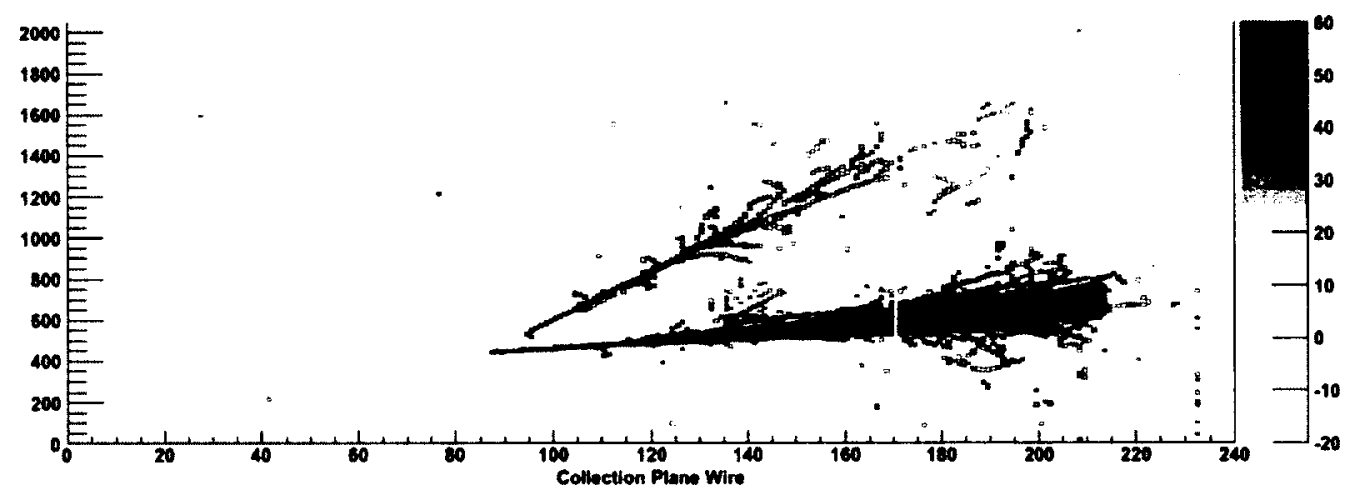

Figure 13.7: The DBSCAN clustering algorithm ran on a neutrino event. The display shows colored hits on the collection plane, each color corresponds to a unique cluster formed. The red hits represent noise as decided by DBSCAN and are not passed to other algorithms.

line that passes through both points as shown in Figure 13.8: The transform employed in LArSoft parametrizes each of the hits given as (wire, time) points that were clustered by DBSCAN and places them in a Hough accumulator $(r, \theta)$ space. The Hough accumulators $r$ and $\theta$ coordinates are discretized in the form of cells of finite height and width [112], and the number of these accumulator cells determine the algorithms resolution. The dimensions of 
the accumulator are decided based on processing time, resolution, and accumulator signalto-noise ratio. The cells with weight above some threshold are selected as line candidates after positioning of all the image points in the discretized Hough accumulator. For the case of search for multiple lines mutually exclusive cells with the largest weight can be considered as lines. After adding all the hits to the Hough accumulator, the cell with the largest number of curve-crossings (weight) is detected. The center-of-mass of the $3 \times 3$ cell window with the weightiest cell at center indicates a candidate line. The center-of-mass of the 9-cell system is used to form the candidate line, instead of the actual central cell coordinate, so that the extracted line parameters are not limited by the cell coordinates discreteness. The process is repeated by considering hits that have not yet been associated with a line until the threshold or a maximum number of lines to be found in a cluster has been reached.

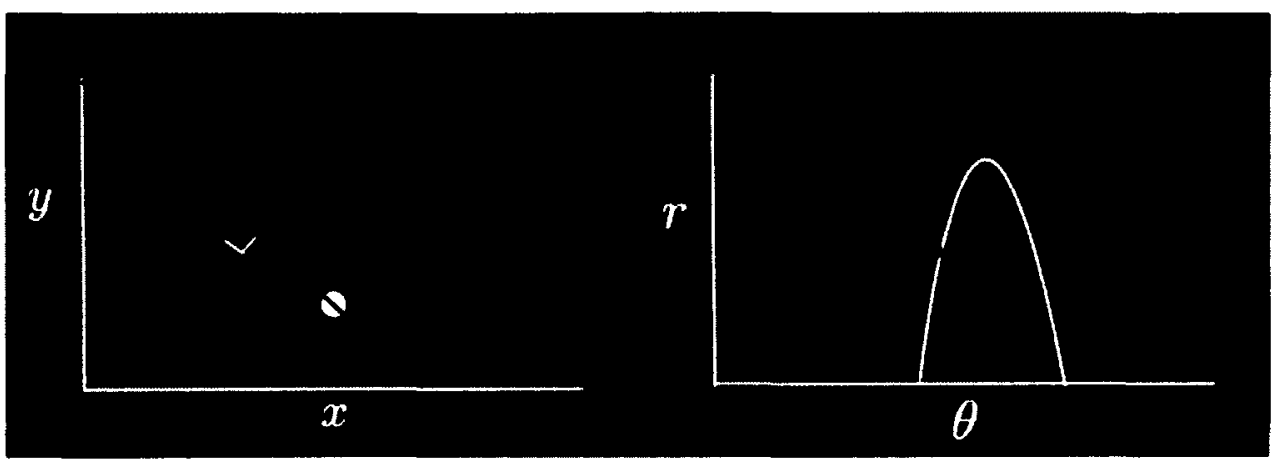

Figure 13.8: Representation of two points in $(\mathrm{x}, \mathrm{y})$ plane and their parametrization in $(\mathrm{r}, \theta)$ space. The crossing curve in $(r, \theta)$ corresponds to the line passing through both points in $(x, y)$.

In case of multiple line-like tracks in an event multiple accumulator convergences are seen and the whole process must be repeated. The cells with weight above some threshold are selected as line candidates after all the image points have been positioned in the discretized Hough accumulator. The algorithm can also smooth the accumulator with a 2D Gaussian convolution to reduce the effect of hits that do not fit well. The Hough line finding algorithm at work for an ArgoNeuT two track neutrino event is shown in Figure 13.9. The algorithm properly separates the two 2-dimensional lines, as seen on a plane, into two clusters.

It is worth noting that the Hough line finding algorithm can often break a single line into multiple lines of slightly different slope and intercepts. This happens if there are more $(\mathrm{r}, \theta)$ cells to choose from the Hough accumulator and is mostly due to multiple scattering. In this case, lines with similar slope and connected endpoints are merged together on a 


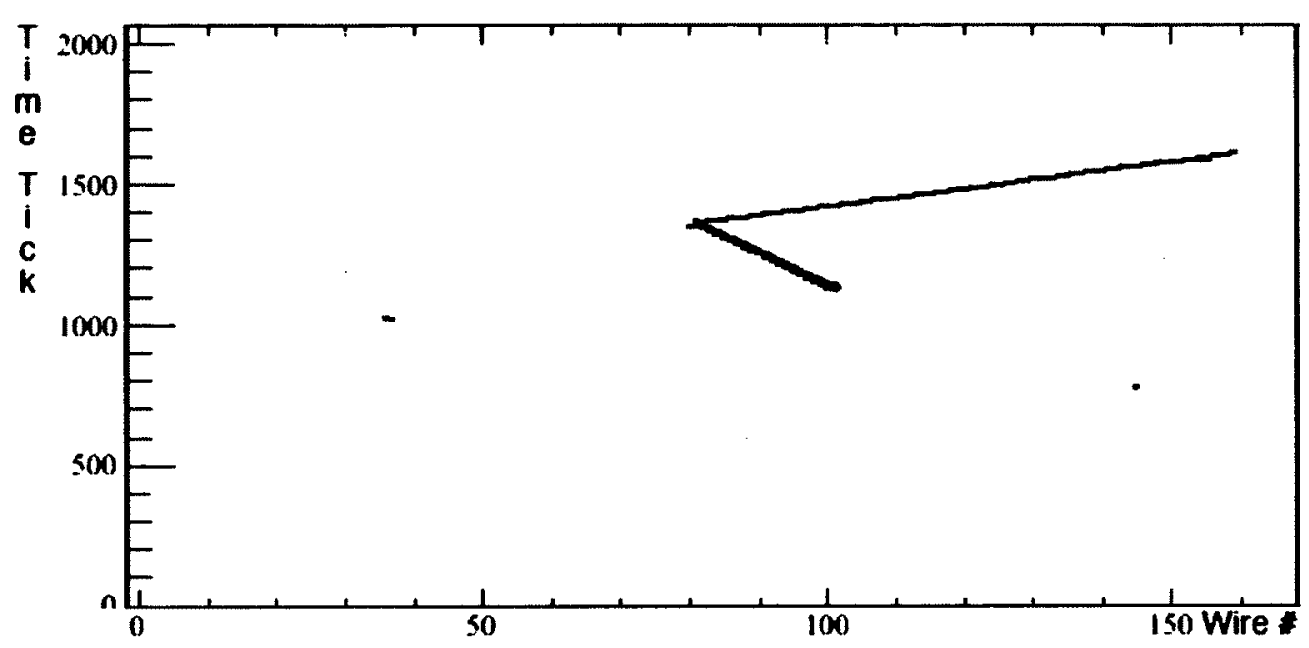

Figure 13.9: 2-dimensional clustering with Hough Transform on a ArgoNeuT neutrino event. The reconstructed hits are visible in gray, while the two colors represent the clustered lines corresponding to 2-dimensional tracks.

plane-by-plane basis in preparation for the 3-dimensional track matching. The output of merging those lines will be referred to as "LineClusters" or "LineMergers" in the Analysis Section.

\subsection{Vertex Finding}

The two dimensional neutrino interaction vertex is found by using the 2-dimensional merged lines in each plane. First, the line-like cluster which is matched with MINOS Near Detector or the longest line-like cluster is identified. The starting point of this cluster in each plane is a guessed vertex in two dimensions. Then all other line-like clusters identified by the Hough Transform are examined. The ones with the point of closest approach to the guessed vertex having a chi-square less than some predetermined tolerance are associated with the vertex. Delta rays can be an inconvenience as they diverge from muons so a protection against them is implemented. Clusters having few hits, a similar slope to the longest cluster, and being far away from the longest cluster starting point are identified as delta rays. Three dimensional vertex is determined after identifying vertices in each plane and matching them in terms of a common drift time. 


\subsection{2-dimensional, More Refined Clustering of Short Vertex Tracks}

An algorithm that can form 2-dimensional clusters for very short tracks present at the vertex is a big challenge. Neither DBSCAN nor any line finding technique currently implemented in LArSoft is efficient enough for this task. An algorithm for forming clusters coming from the vertex was implemented and called VCluster (referred to as "KingaCluster in LArSoft). The algorithm takes as an input hits that were clustered by DBSCAN. It also relies on having a reconstructed vertex from the previous algorithm. The 2-dimensional vertex is taken as the origin, and for each hit theta angle is calculated as shown in Figure 13.10. Then a distribution of number of hits versus theta angle can be made. An example of a 2-track data event with this distribution is shown in Figure 13.11. Two distinct peaks are seen. The task is to assign hits belonging to the first peak to Cluster 1 and the hits belonging to the second peak to Cluster 2. The task is not a hard one when tracks are long and distinct peaks are formed. However, it becomes challenging when the track length consists of very few hits. There are also hits that are not falling into neither peak. An algorithm defining a start and end point for these peaks on an event-by-event basis is implemented. This algorithm also decides on a height that a peak must have in order to be considered a separate cluster. A very low threshold for peak's height must be assigned in order to form a separate clusters for short tracks. This threshold was tuned on data events and examples of this algorithm at work for ArgoNeuT neutrino events and comparison with LineMerger is shown in Figures 13.12- 13.14. In each case shown VCluster is able to better separate or create clusters for tracks originating at the vertex than LineMerger. It should be noted that this algorithm does not introduce additional noise hits and all hits clustered by DBSCAN are clustered as well. 


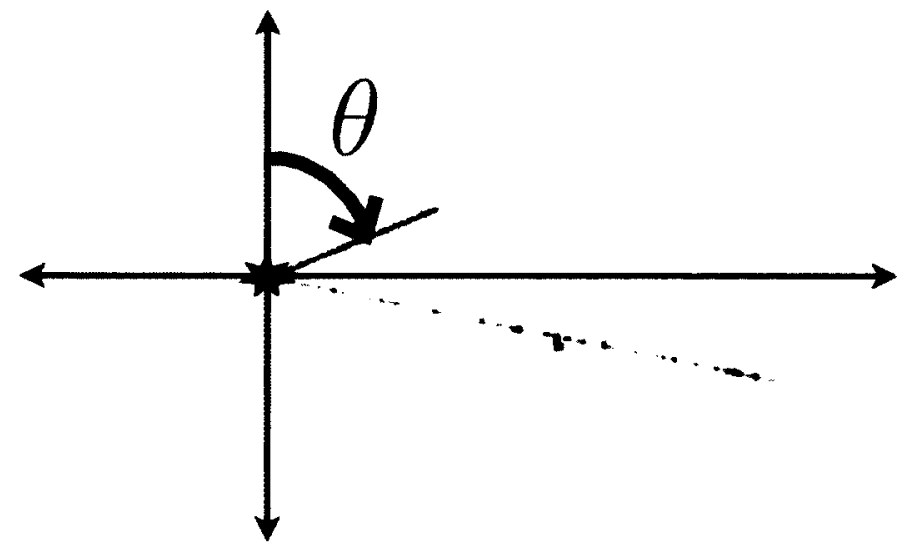

Figure 13.10: A 2-track data event shown in collection plane. Origin of the coordinate system is set on a reconstructed vertex, each hit reconstructed by DBSCAN has its angle theta calculated as indicated.

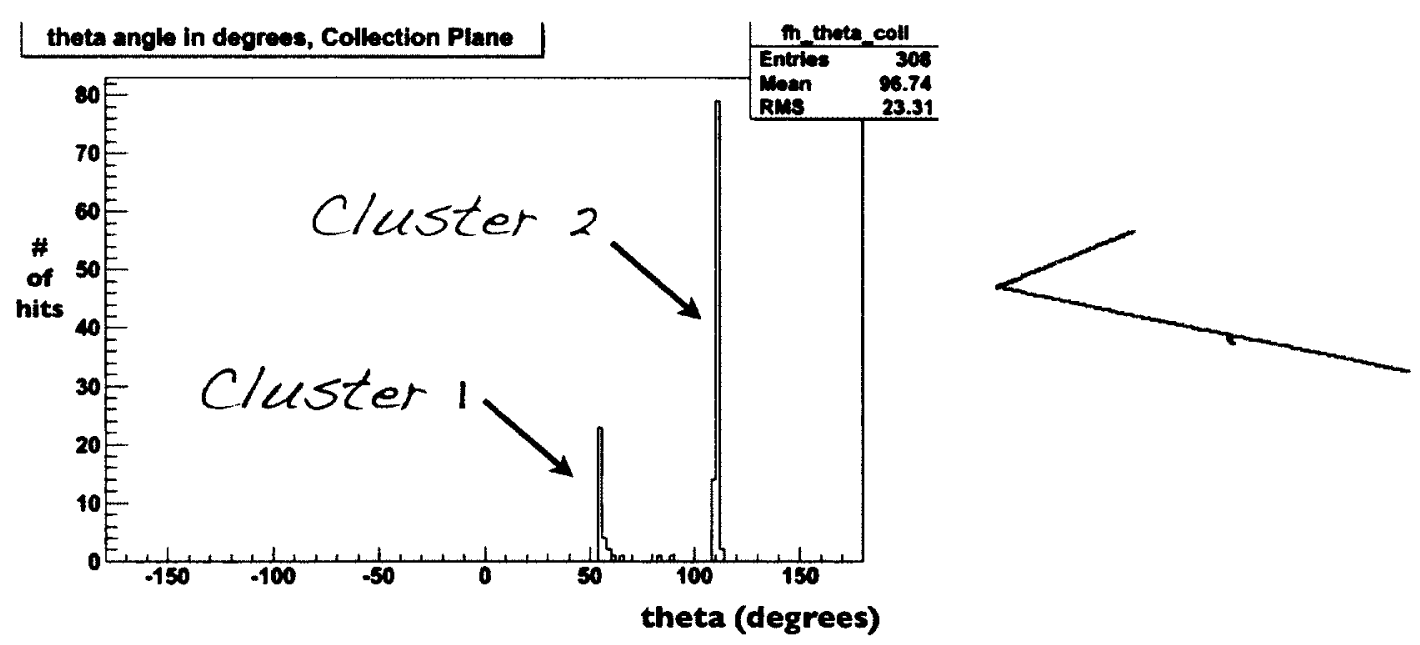

Figure 13.11: A plot of number of hits as a function of theta angle performed for the data event shown on the right. Clusters are formed from the two peaks clearly visible in the plot. The hits not falling into either cluster are assigned based on their proximity. The colored hits correspond to individual clusters formed (green and red). 

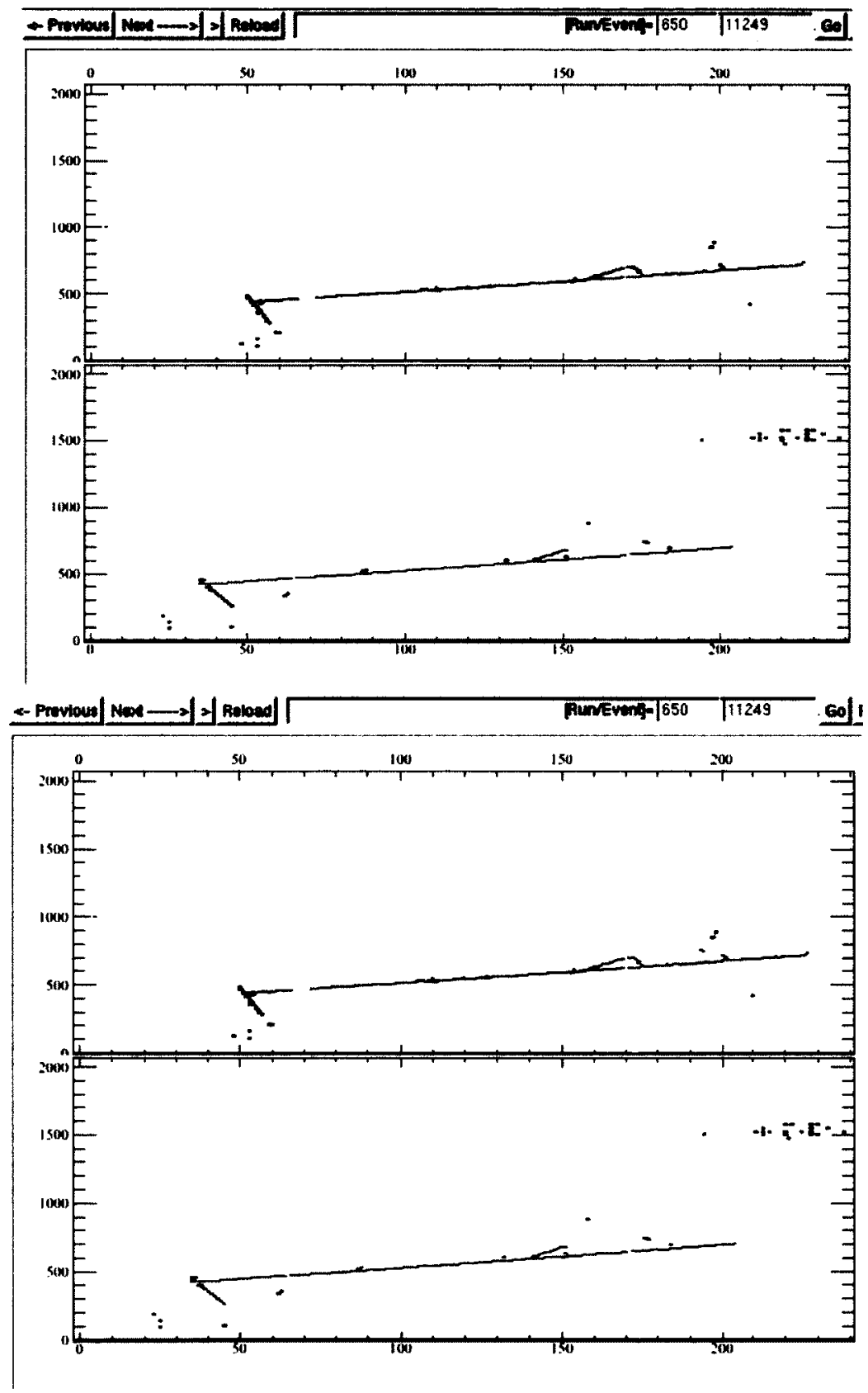

Figure 13.12: The event display showing LineMerger (top) and VCluster (bottom) ran on the same data event. In this case LineMerger merges two short tracks as one on top plane and fails to recognize a 2 -hit cluster on the bottom plane while VCluster reconstructs both. 

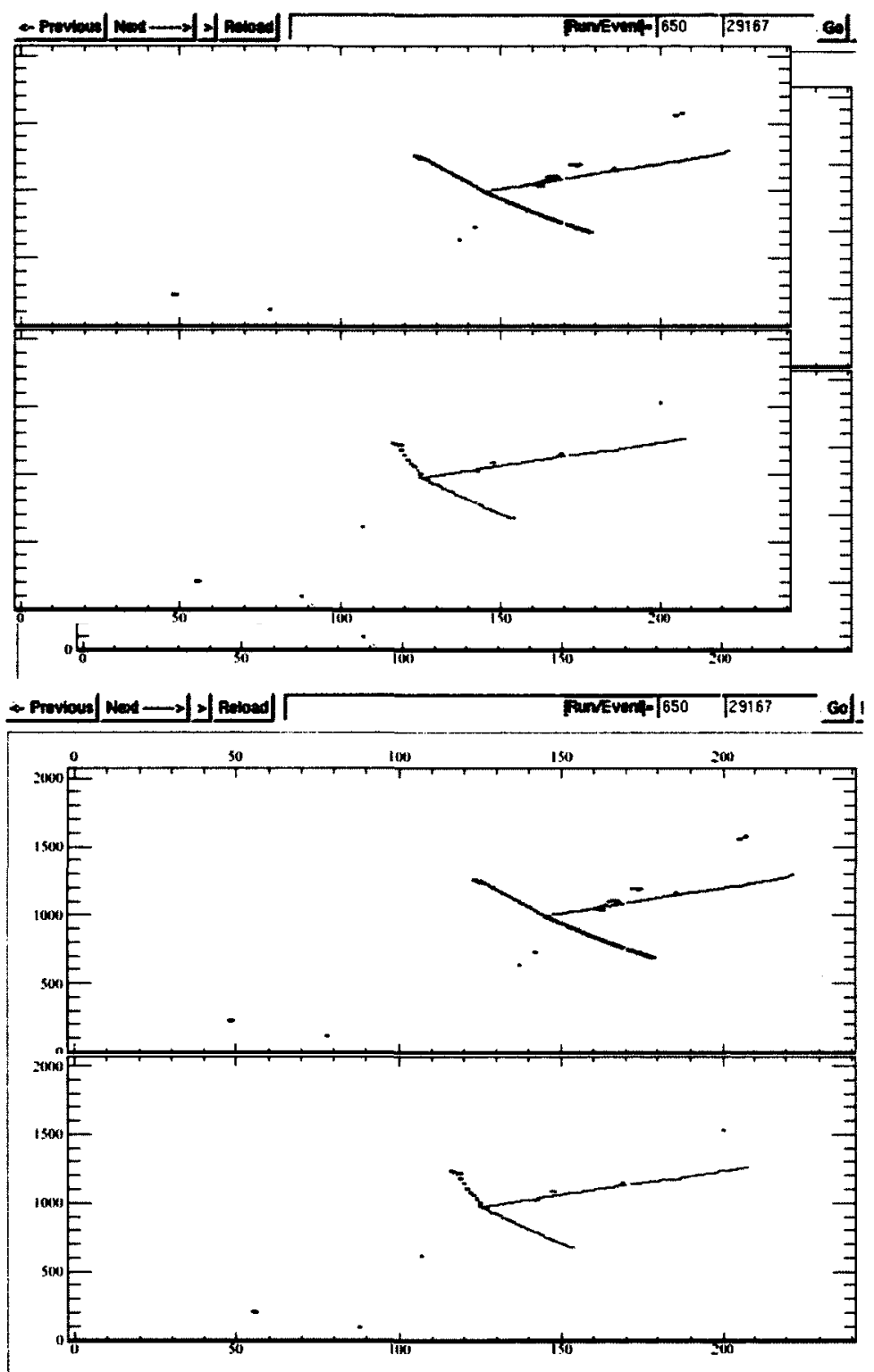

Figure 13.13: The event display showing LineMerger (top) and VCluster (bottom) ran on the same data event. In this case LineMerger merges two tracks as one on top plane while VCluster reconstructs them as separate clusters. 


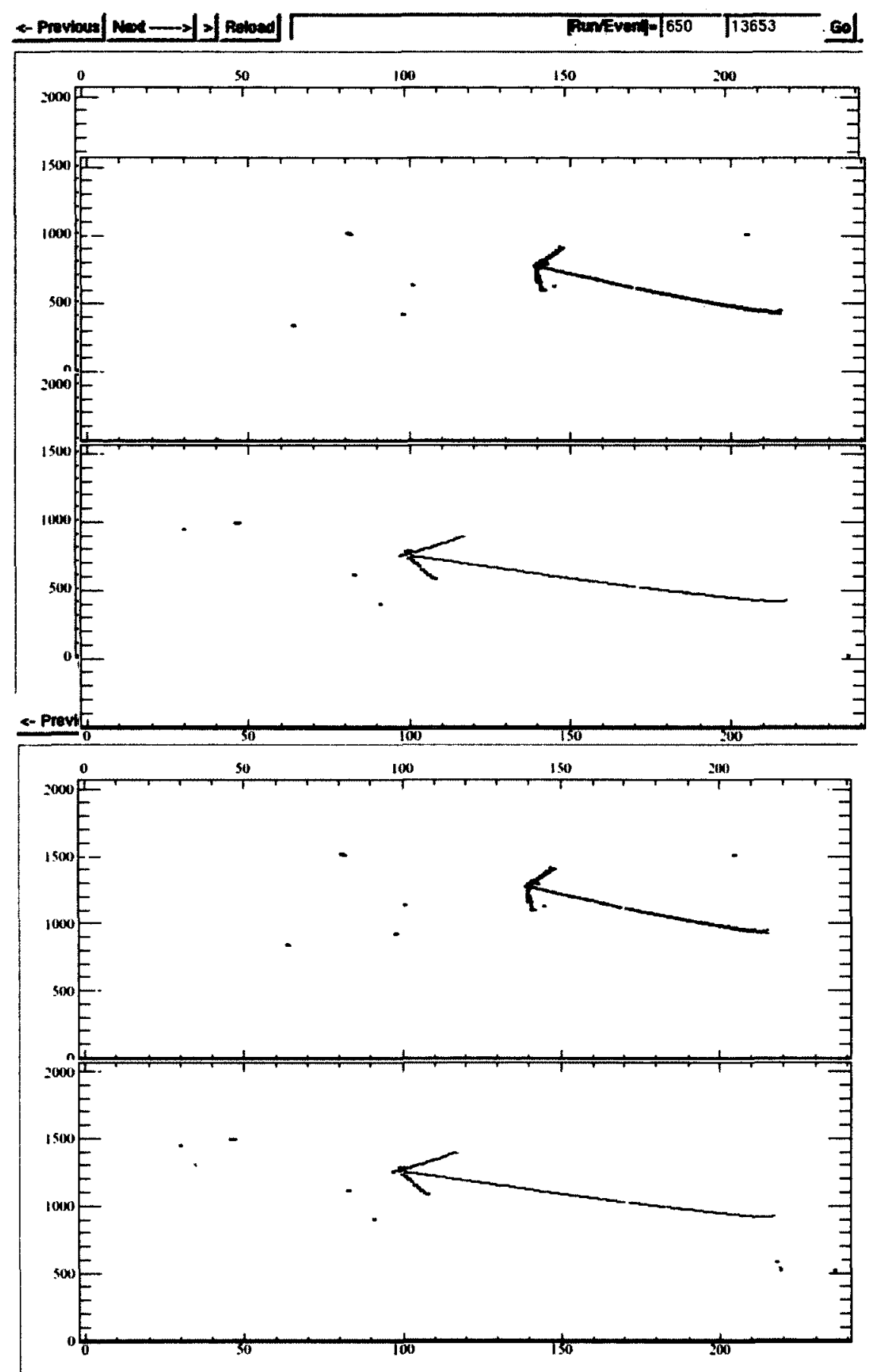

Figure 13.14: The event display showing LineMerger (top) and VCluster (bottom) ran on the same data event. In this case LineMerger does not reconstruct the short track coming from the vertex in the top plane while VCluster forms a cluster. 


\subsection{3-dimensional Track Reconstruction}

The goal of 3 dimensional reconstruction software is to combine 2-dimensional clusters in each plane view in order to create a $3 \mathrm{D}$ image of an event. The 2-dimensional line-like clusters after merging (if any) are passed to the 3-dimensional reconstruction. The main association criteria requires the line-like clusters to have endpoints with the same drift coordinates in the two views. After accounting for the drift distance between the Induction and Collection planes, a time window tolerance for the common time is defined. Track pitch length, necessary for the calorimetric reconstruction, and directional cosines for each track are reconstructed. Track pitch length is defined as the effective length of the portion of a track that is exposed to a single wire and depends on the orientation of the track with respect to the direction of the wires in the plane. After 3D track identification a hit-byhit association procedure is applied to match hits from the two wire planes to obtain a fine-grained 3D image of the event as shown in Figure 13.15.

A dedicated matching algorithm is employed to create $(x, y, z)$ points that are referred to as space points. They are associated to the wire ( $\mathrm{v}$ for Induction and $\mathrm{w}$ for Collection planes) and drift time (t) by the following relations:

$$
\left.\begin{array}{r}
x=t * v_{d} \\
y=\frac{w-v}{2 * \cos (\alpha)} \\
z=\frac{w+v}{2 * \sin (\alpha)}-\frac{h}{2 * \tan (\alpha)}
\end{array}\right\}
$$

where $v_{d}$ is the drift velocity in the argon, $\alpha$ is the absolute value of the orientation angle of the wires in the wire planes and $h$ is the TPC box's height. The drift time is corrected for pre-sampling as well as offsets due to the longer drift distance to the Collection plane.

\subsection{Calorimetric Reconstruction}

The geometrical reconstruction of the 3-dimensional tracks and the space-points, described above, are preliminary steps for calorimetric reconstruction. At this point, the hit amplitude, hit time coordinate and track pitch length for all hits belonging to a reconstructed track are known. Calorimetric measurements are made using the charge collected by the collection plane as it provides a higher gain. 


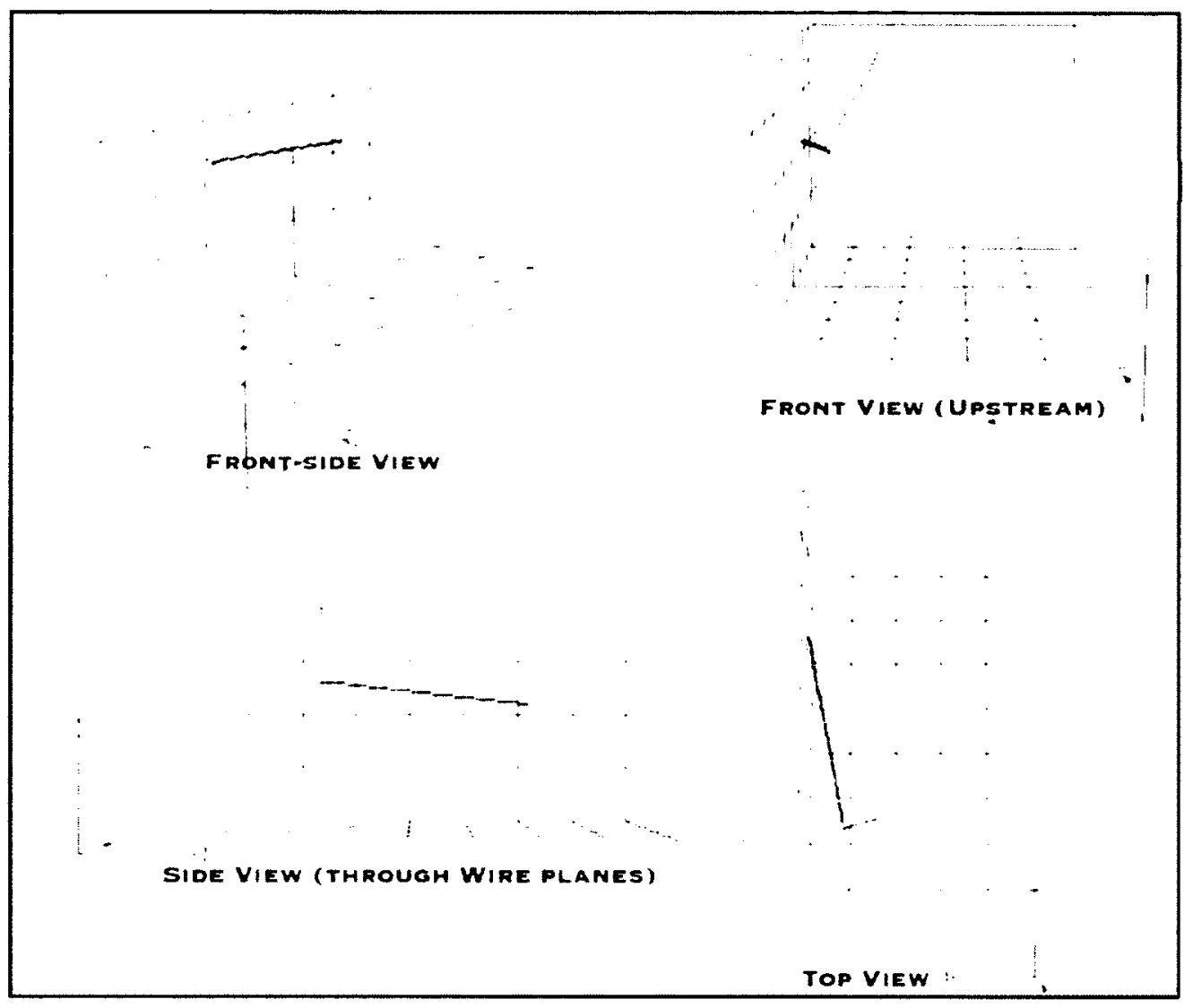

Figure 13.15: Different views of a reconstructed neutrino event in three dimensions. The longest track is seen to be exiting the TPC volume through the cathode plane.

The energy loss of charged particles, as they transverse the liquid argon medium, varies with a particle type and energy. Unfortunately, not all the released charge survives due to recombination effect [92]. This effect occurs before the opposite charges are separated by the applied electric field and grows with local ionization density. It is often referred to as 'charge quenching'. Free electrons are then uniformly transported along the electric field lines.

Another loss of free electrons can occur due to electron attachment to electro-negative impurities present in liquid argon. The hits are recorded for all the charge that makes it to the Induction and Collection wire planes. Corrections for these two effects need to be applied in order to obtain the amount of total charge released. First, a correction to obtain the free charge after recombination is applied as $Q_{f r e e}=Q / e^{-t / \tau_{e}}$, where $\mathrm{t}$ is the hit time and $\tau_{e}$ is the electron lifetime measured by ArgoNeuT. The second correction 
applied to correct for the recombination effect and thus obtain the initial released charge is $Q_{\text {init }}=Q_{\text {free }} / R$ where $\mathrm{R}$ is the recombination factor derived from a parametrization of the quenching effect in liquid argon and based on Birk's model [113].

The initial released charge in the track pitch is directly proportional to the energy deposited. The energy loss along the track $(\mathrm{dE} / \mathrm{dx})$ can be estimated in steps of track pitch length and then summed in order to obtain the total deposited energy.

The energy loss as a function of residual range is a powerful particle identification technique for particles that stop inside the liquid argon volume. The residual range is a path length to the end point of the track. This technique can be used as particles have different energy loss along the track as they slow down and come to a stop as shown in Figure 13.16 (left). The curves of different particles come from a GEANT simulation of stopping particles. An example of this technique used on the data is shown in Figure 13.16 (right) for the stopping track in ArgoNeuT. The black points represent the hits along the track, it is seen that they are aligned nicely on the proton hypothesis curve. The reconstructed track length also agrees with the total deposited energy obtained from the calorimetric reconstruction.
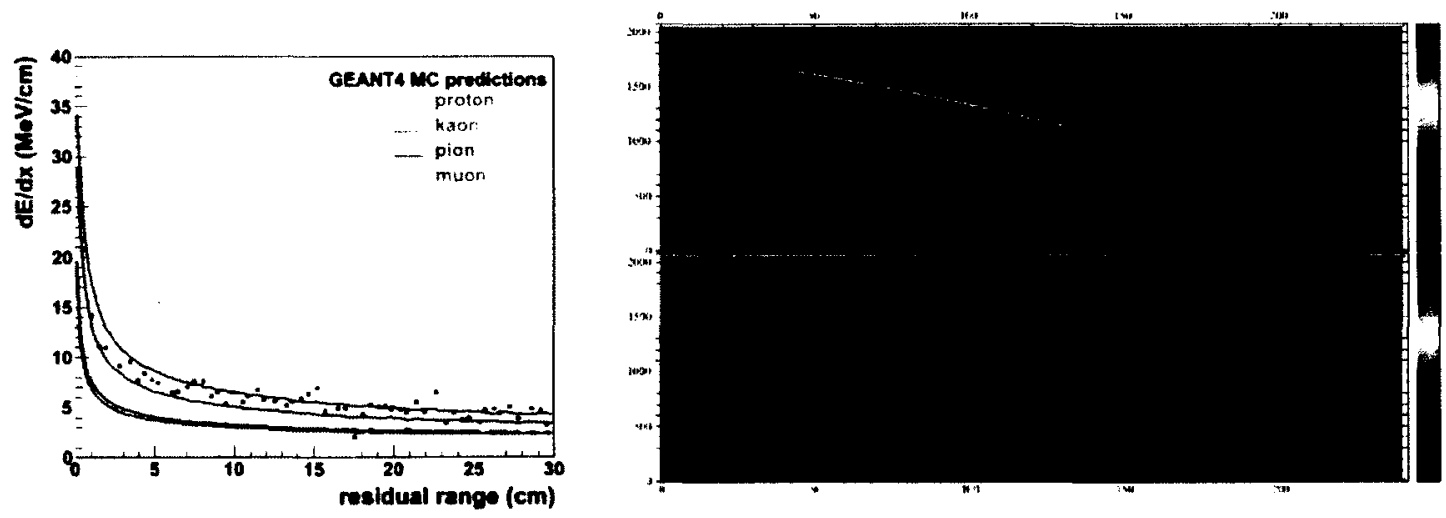

Figure 13.16: Simulated energy loss per unit track length $(\mathrm{dE} / \mathrm{dx})$ as a function of residual range (distance to the track end) for different stopping particles. The black points correspond to the experimental data for the hits along the track shown on the right. They nicely agree with the proton hypothesis.

\subsection{Matching with MINOS Near Detector}

Most muons originating in ArgoNeuT's TPC will exit due to the detector's small size and enter MINOS Near Detector (MND). Track reconstruction in MND is performed by 
MINOS off-line analysis code [114] and the results are provided directly by the MINOS experiment. The track momentum is estimated from range if the track stops within the detector, or from a measurement of its curvature in the MINOS-ND toroidal magnetic field if it exits. The curvature measurement is obtained from fitting the trajectory of the track using Kalman filter techniques that take into account bending of the track from both multiple Coulomb scattering and the magnetic field. This procedure also determines the charge of the reconstructed track. In order for a track to be considered a candidate for ArgoNeuT-MINOS matching it must have a reconstructed $\mathrm{z}$ vertex less than $20 \mathrm{~cm}$ from the center of the first MINOS plane. Tracks exiting ArgoNeuT and entering MND are preselected for matching based on a spill-by-spill basis by knowing the common timestamp from the accelerator division. The actual position and orientation of the tracks relative to one another further defines the matching. The two requirements for the geometrical matching are:

- the radial difference between the exiting ArgoNeuT track projected onto MND first plane and the MINOS entering track vertex must be less than $25 \mathrm{~cm}(\Delta r)$

- the cosine angle between the ArgoNeuT track direction at TPC exit and the MINOSND track at vertex must be less than $0.4 \mathrm{rad} .(\cos \theta)$

The schematic illustrating the relative size of the two detectors, their position, coordinate systems and the matching scenario is shown in Figure 13.17. In the event of multiple matches the best pair combination that minimizes $\Delta r / \cos \theta$ quantity is chosen. More details on the matching for $\mu+N p$ analysis and comparison plots between MC and data are shown in Section 14.2.1. It should be noted that the MINOS reconstructed variables are interpreted by the ArgoNeuT and may not be representative of the MINOS experiment as different requirement have been applied, such as goodness-of-fit requirements and different cuts. 

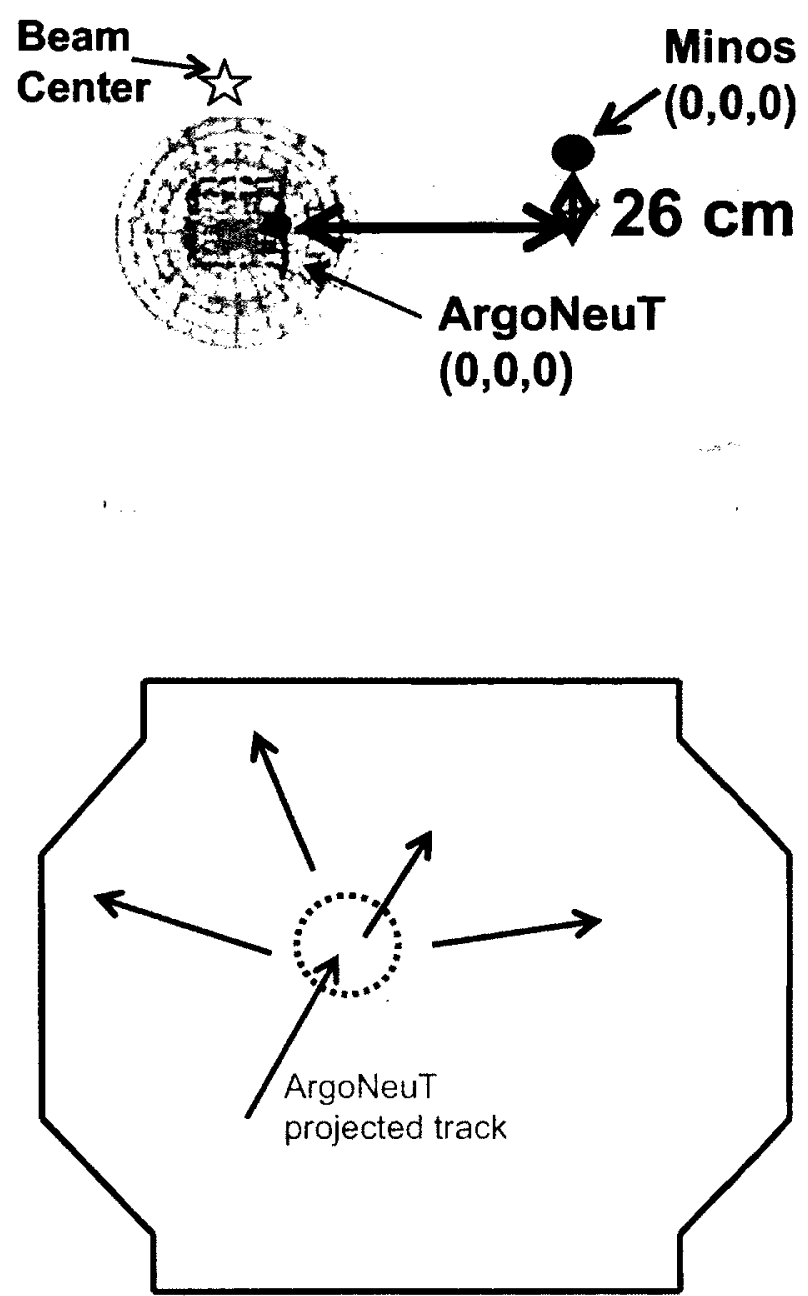

Figure 13.17: The front view of the ArgoNeuT and MINOS detectors from the GEANT4 Simulation (top). The ArgoNeuT TPC is shown in pink. The beam center, origin of the MINOS and ArgoNeuT coordinate system is shown. A matching muon scenario is shown where one track in ArgoNeuT has multiple candidate tracks from MINOS (bottom). 


\section{PART V}

Analysis 


\section{Analysis of $\mu+\mathrm{Np}$ Neutrino Interactions}

\subsection{Introduction and Importance of $\mu+N p$ Topological Measurement}

Qusi-Elastic scattering is a process which is a signal for most oscillation experiments and have been called a "golden" channel to due it's simple topology. According the the Monte Carlo, it composes the biggest fraction of the $\mu+N p$ events presented in this thesis. The Charged Current Quasi-Elastic (CCQE) interaction for neutrinos involves conversion of neutron into proton. For anti-neutrinos, on the other hand, proton is converted into neutron:

$$
\begin{aligned}
& \nu_{\mu}+n \rightarrow \mu^{-}+p \\
& \bar{\nu}_{\mu}+p \rightarrow \mu^{+}+n
\end{aligned}
$$

Even though it seems simple, it is truly more complex than previously envisioned due to nuclear effects that can change what is emitted in the final state. Great care must be taken in actually defining what "CCQE" means as each experiment can have different definitions depending on what they can detect in the final state. Most detectors cannot detect low energy particles at the vertex so their definition might be more inclusive. As an example, MiniBooNE selection consists of events with a muon and no pions. NOMAD, on the other hand, selects only one or two-track events. It is not clear what fraction of events accepted by MiniBooNE are rejected by NOMAD [116]. Thus, the definition of CCQE is highly subjective. It may be that different definitions of CCQE lead to different results. Due to this ill-defined process, the analysis presented here focuses on topological measurement of $\mu+N p$ events. This way it is clear what the final state products are: muon and any number of protons. 
Furthermore, the topological analysis presented here avoids large corrections based on Monte Carlo, such as pion background as will be discussed in this analysis section. It is noted in [116] that estimates of single pion backgrounds have large $(>10 \%)$ uncertainties associated with them. These estimates are made from some combination of simulations and data-driven methods incorporating additional events samples.

The analysis presented in this thesis is the first topological analysis in neutrino physics that can fully reconstruct and count protons with a low threshold of $21 \mathrm{MeV}$ of kinetic energy. It takes full advantage of the LArTPC technology by making this measurement. Cherenkov based detectors, such as MiniBooNE, are not sensitive to final state nucleons and thus must heavily rely on a Monte Carlo to further constrain their event sample. Due to the complicated nature of the neutrino interaction with a heavy nuclei, one should avoid making Monte Carlo corrections where possible. As noted earlier, Final State Interactions (FSI) and other nuclear processes can drastically alter what is present in the final state. With this in mind, one should make a measurement based on what is 'seen' in the final state and have a clear definition of a process subject to a measurement. Liquid Argon detectors are very well suited for that task due to bubble-chamber-like quality, excellent particle identification and background rejection. Due to the imaging capabilities of LArTPCs one can reconstruct what is present in the final state after FSI, for example, muon and any number of protons, muon and any number of pions, and so forth and thus separate analyses based on a final state.

By a minimal reliance on $\mathrm{MC}$, the $\mu+N p$ analysis presented here includes all nuclear processes of neutrino-nucleus scattering. The multi-nucleon emission is clearly visible with proton multiplicity up to and including 4 . The rates of proton multiplicity are the first measurement in neutrino physics and can be used to tune Monte Carlo generators and check varies theories. The proton kinematics plots with such a low threshold are also first measurements that will serve to further understand nuclear processes. It should be noted that $\mu+N p$ events entering the final analysis sample can contain any number of neutrons as ArgoNeuT is too small to contain the protons coming from neutron re-interactions.

The $\mu+N p$ analysis will make a use of visual scanning, that is looking at each event to carefully study its feature. The implementation of this method is quite unusual, as the current neutrino experiments do not utilize such a tool in their analyses and are mostly based on fully automated reconstruction. However, due to bubble-chamber like quality of ArgoNeuT, scanning presents a very powerful tool. Though time consuming, the writer be- 
lieves it is the most powerful tool that allows to learn about features of neutrino interactions that have not been possible to explore with other technologies and existing experiments. Since the work presented here explores protons around a vertex in $\mu+N p$ interactions, it requires a great care as the protons might be very short, as will be shown.

Visual scanning helps to guide the reconstruction algorithms. The knowledge gained from visual study of neutrino interactions, first without use of any reconstruction tools, will also tremendously help to write a more sophisticated algorithms in the future. It should be noted, that this study is possible in ArgoNeuT since the smaller number of data events allows for careful exploration. The hope is to be able to write algorithms that could perform similar topological analysis with much bigger statistics, for example with the use of MicroBooNE, by using the knowledge gained from visual scanning from the presented here analysis.

\subsection{Analysis Steps}

The aim of this analysis is to study events with a muon and any number of protons at a vertex. The analysis has utilized about five months of ArgoNeuT physics run in antineutrino mode, both neutrinos and anti-neutrinos will be studied. The energy distribution for neutrinos and anti-neutrinos is shown in Figure 14.1, it is seen that anti-neutrinos cover a much lower energy energy spectrum with respect to the neutrinos. The energy spectrum for $\mu^{-}+N p$ events and comparison with the energy distribution for all neutrino events is shown in Figure 14.2. The energy spectrum for $\mu^{+}+N p$ events and comparison with the energy distribution for all antineutrino events is shown in Figure 14.3. The $\mu^{-}+N p$ have a lower mean value of energy when compared with all the neutrino events. Similarly, $\mu^{+}+N p$ have a lower mean value of energy when compared with all the anti-neutrino events in anti-neutrino mode.

The selection procedure for this topological analysis proceeds first with automated reconstruction. Its goal is to reject as much background as possible while keeping the sample of interest large. The automated reconstruction selects only events in which the muon track in ArgoNeu'T is matched to the track in MINOS Near Detector (MND). This is necessary in order to obtain the muon sign and momentum. The sign selection allows for splitting the $\mu+N p$ sample into events coming from neutrino interaction, $\mu^{-}+N p$, and anti-neutrino interactions, $\mu^{+}+N p$. In addition to the matching of the muon with MINOS, there are a 


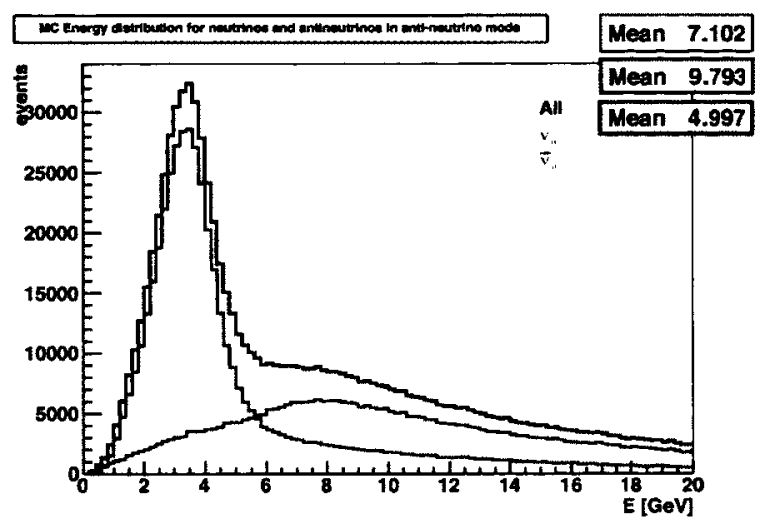

Figure 14.1: The Monte Carlo simulation of the energy spectrum for neutrinos and antineutrinos in anti-neutrino mode in the NuMI beam line.

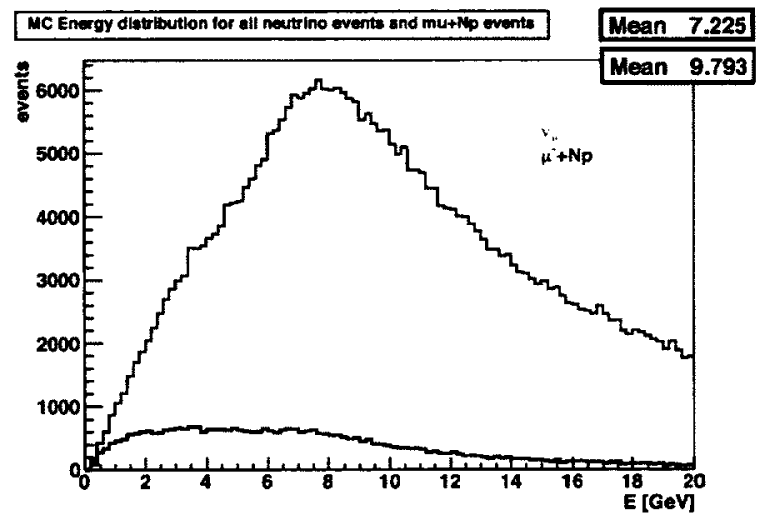

Figure 14.2: The Monte Carlo simulation of the energy spectrum for neutrinos and $\mu^{-}+N p$ events.

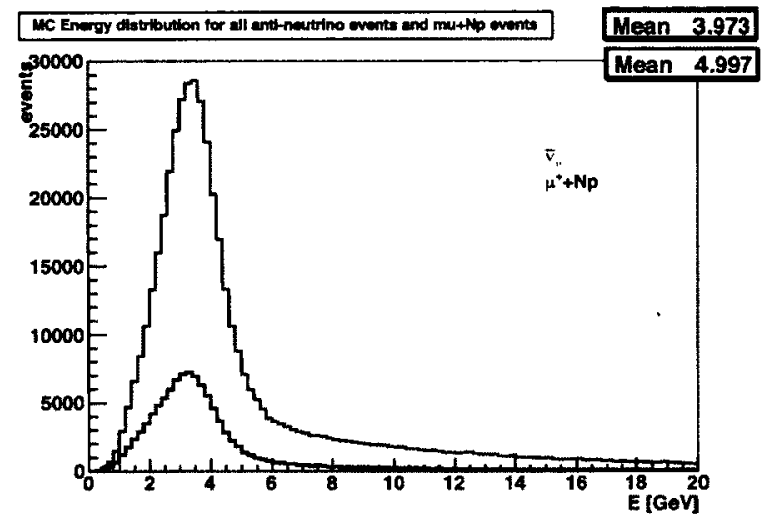

Figure 14.3: The Monte Carlo simulation of the energy spectrum for antineutrinos and $\mu^{+}+N p$ events. 
number of cuts used to further restrain the sample. The details of the algorithms used in the automated reconstruction are described in Chapter 13, the specific cuts used to select the $\mu+N p$ events are described in Section 14.2.1.

After fully automated reconstruction, the sample selection is further developed by the use of visual scanning. This is necessary as we must first understand the details of these interactions by carefully examining them visually before high level algorithm can be developed. Visual scanning procedure is described in Section 14.2.3.

The last step is calorimetric reconstruction on the sample selected in the visual scanning process. This reconstruction proceeds in semi-automated way, in which a scanner guides the automated three-dimensional reconstruction, described in Section 13.7, by manually providing hits belonging to a candidate proton track. Then, the calorimetric reconstruction uses the formed track to perform particle identification as described in Section 14.2.5.

\subsubsection{Setting the Automated Cuts}

The automated cuts discussed below have been set in order to minimize the background while maintaining high statistics and efficiency. However, due to very time consuming nature of visual scanning and calorimetric reconstruction, which are the steps that follow automated cuts and are discussed in detail in Section 14.2.3 and 14.2.5, the automated cuts have been focused on picking events with lower number of tracks.

Automated cuts consist of the following requirements:

1. vertex and the start position of the track matched with MND must be inside fiducial volume. Fiducial volume is defined to be a region $3 \mathrm{~cm}$ off the TPC wall in $\mathrm{x}$-direction, $4 \mathrm{~cm}$ in $\mathrm{y}$-direction, $6 \mathrm{~cm}$ in z-upstream and $4 \mathrm{~cm}$ in z-downstream.

2. exactly one track must be matched with $\mathrm{MND}$, have momentum $>0$ and appropriate sign ( + for anti-neutrino and - for neutrino analysis)

3. Three additional automated cuts on the number of clusters in each view and the number of exiting tracks will be imposed:

$$
\begin{aligned}
& 1<=\text { number of vertex VClusters }<=2 \\
& 1<=\text { number of vertex LineMergers }<=2 \\
& 1<=\text { number of exiting } 3 \mathrm{~d} \text { tracks }<=2
\end{aligned}
$$


Three main criteria used for setting the fiducial volume cut are: preventing electric field non-uniformities near the edges of the TPC, preventing misidentifying a through-going muon as a neutrino-induced muon, and maintaining a large event sample. The difference between the reconstructed neutrino vertex and the true vertex together with a two dimensional plot for each coordinate is shown in Figure 14.4 for anti-neutrinos and in Figure 14.5 for neutrinos. The truth information comes from the GENIE event generator while "reco" refers to reconstruction applied to the simulated neutrino event. Events that enter these plots are true $\mu+N p$ events that pass all the automated cuts listed above. In order to understand the vertex resolution, a Gaussian fit is applied to each difference between reconstructed and true vertex distribution. Long tails are especially significant for $\mathrm{Y}$ and $\mathrm{Z}$ distributions that are caused by mis-reconstructed space-points, which resulted from mismatched hits in two planes. However, it should be noted that vertex resolution is at the sub-cm level. The shift in the $\mathrm{X}$ coordinate is expected as the start time of a MC event is anywhere within the NuMI beam window of $10 \mu \mathrm{s}$. Therefore, the reconstructed $\mathrm{x}$ distance will always be later than $t=0$ by some random, uniform amount given by the drift velocity $x$ this time and can be as much as $1.6 \mathrm{~cm}$.

The nominal cuts have been adopted from the Charged Current inclusive analysis [117]: $4 \mathrm{~cm}$ in the $\mathrm{Y}$ and $\mathrm{Z}$ coordinates employed to avoid electric field non-uniformities. Additional distance of $2 \mathrm{~cm}$ is subtracted from the upstream $\mathrm{Z}$ edge of the detector to avoid throughgoing muons entering the sample. Not all of them are eliminated by this cut which can be seen by the high number of events at low- $Z$ in Figure 14.6. All events passing automated cuts and appropriate selection for the muon sign (neutrinos on left and anti-neutrinos on right) enter this sample. The excess of events at low- $Z$ is not seen in the $M C$ as throughgoing muons are not simulated. The reason for their presence in the data is their vertex mis-reconstruction stemming from varying electric field near the boundaries of the TPC which is not incorporated in the MC simulation and low electron lifetime. These effects cause the vertex to be reconstructed more inside the TPC. However, these through-going muons are eliminated in the visual scanning stage. The area normalized plots of distribution of vertices after elimination of through-going muons in the data and comparison with the Monte Carlo are shown in Figure 14.8. The effect of reconstructing vertex more inside the TPC is especially significant for anti-neutrinos in the drift coordinate.

The vertex distribution of data events passing all automated cuts for anti-neutrino mode (neutrinos and anti-neutrinos together) in $\mathrm{Y}$ vs. $\mathrm{X}$ and $\mathrm{Z}$ vs. $\mathrm{X}$ is shown in Figure 14.7. The 

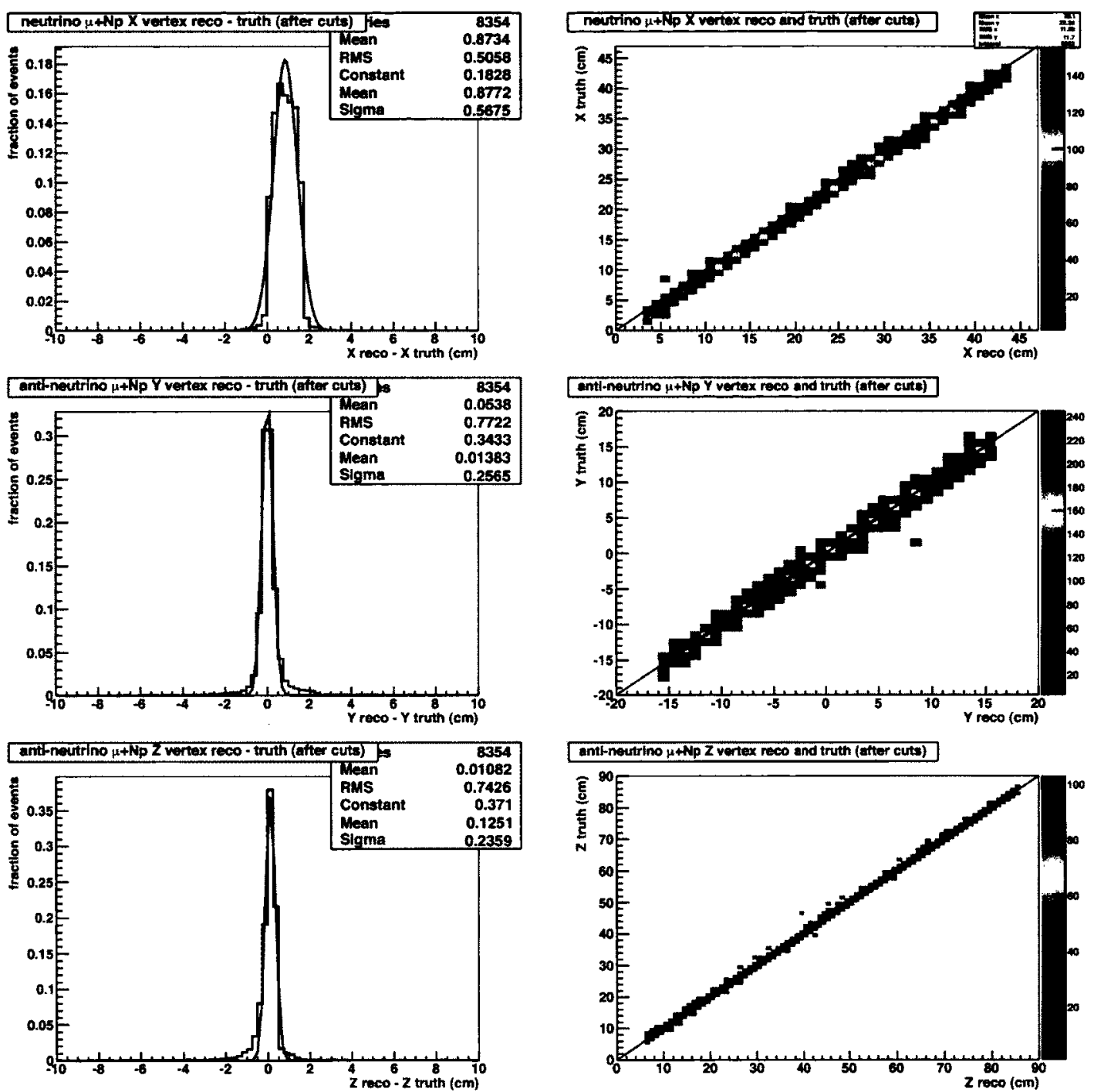

Figure 14.4: Comparison of true and reconstructed value of the $\mathrm{X}, \mathrm{Y}$, and $\mathrm{Z}$ vertex coordinates for $\mu^{+}+\mathrm{Np}$ events that pass all automated cuts.

active volume of the TPC is represented by the axis range and the fiducial cuts performed can clearly be seen. There are some through-going muons present in this plot as noted earlier.

\section{Matching Exiting Muons with MINOS Near Detector (MND)}

ArgoNeuT sat in front of MINOS Near Detector (MND), which provides the reconstruction of muon sign and momentum. In order for this to be accomplished, muons that exit the 

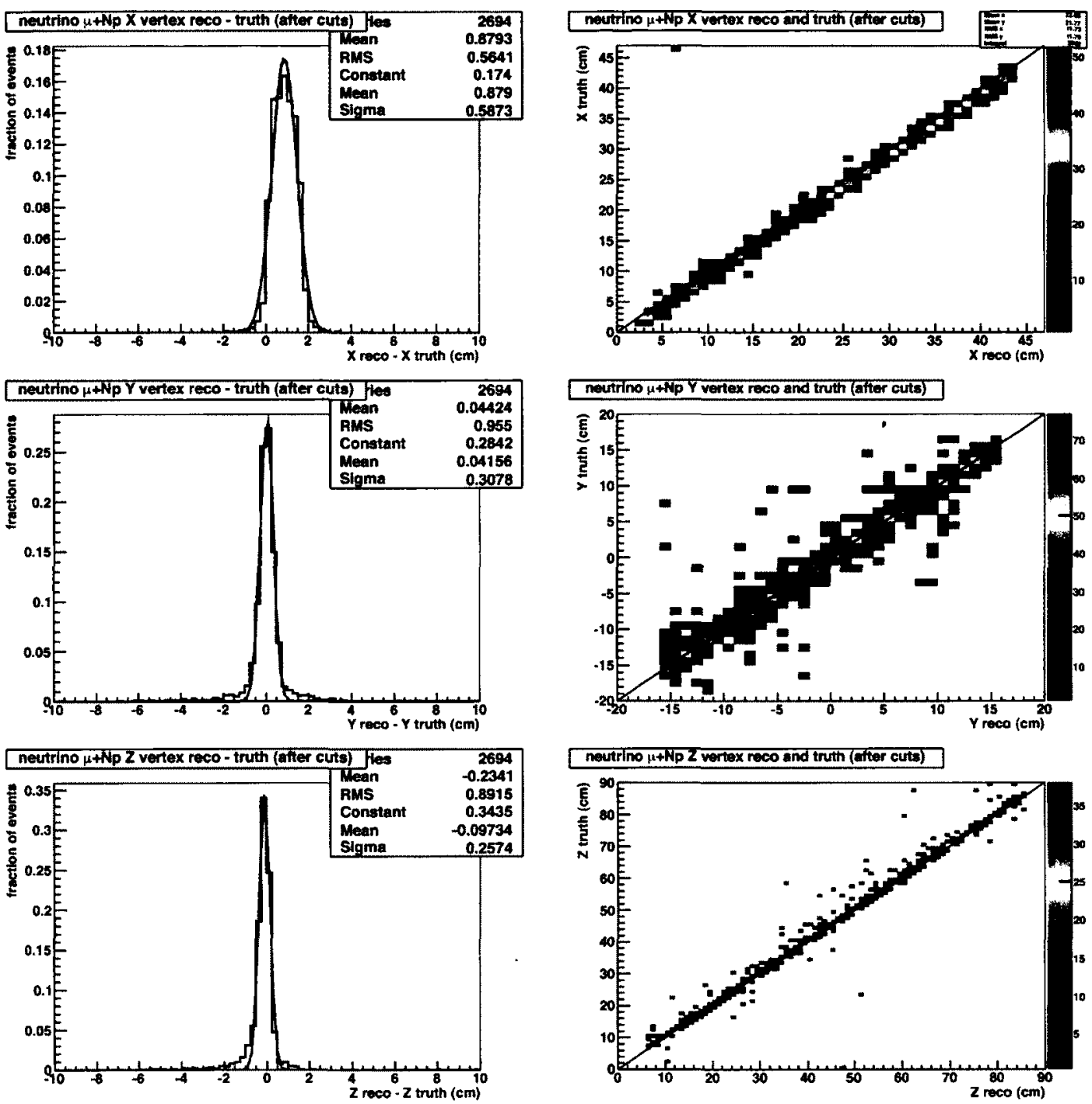

Figure 14.5: Comparison of true and reconstructed value of the $\mathrm{X}, \mathrm{Y}$, and $\mathrm{Z}$ vertex coordinates for $\mu^{-}+\mathrm{Np}$ events that pass all automated cuts.

TPC need to be reconstructed and matched with the corresponding track in MINOS. Only events for which a muon is matched in MINOS are considered in this analysis. A schematic of the relative size of the two detectors and positioning is shown in Figure 14.11. As can be seen ArgoNeuT is tiny compared to MINOS and due to their relative positioning it can be expected that most muons that exit the TPC will enter MINOS. Figure 14.12 shows a negatively matched track with MINOS and also other tracks that were present during this spill. 

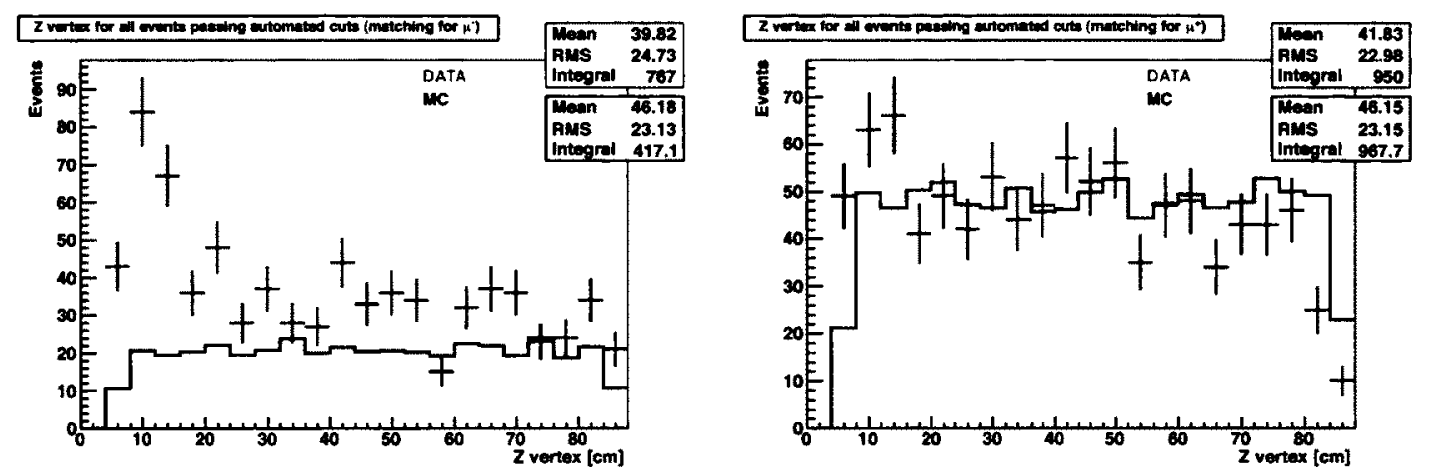

Figure 14.6: The distribution of vertices for all data events that pass automated cuts. Neutrinos on the left and anti-neutrinos on the right. There are through-going muons entering the upstream end of the TPC which are eliminated later in the process of visual scanning.
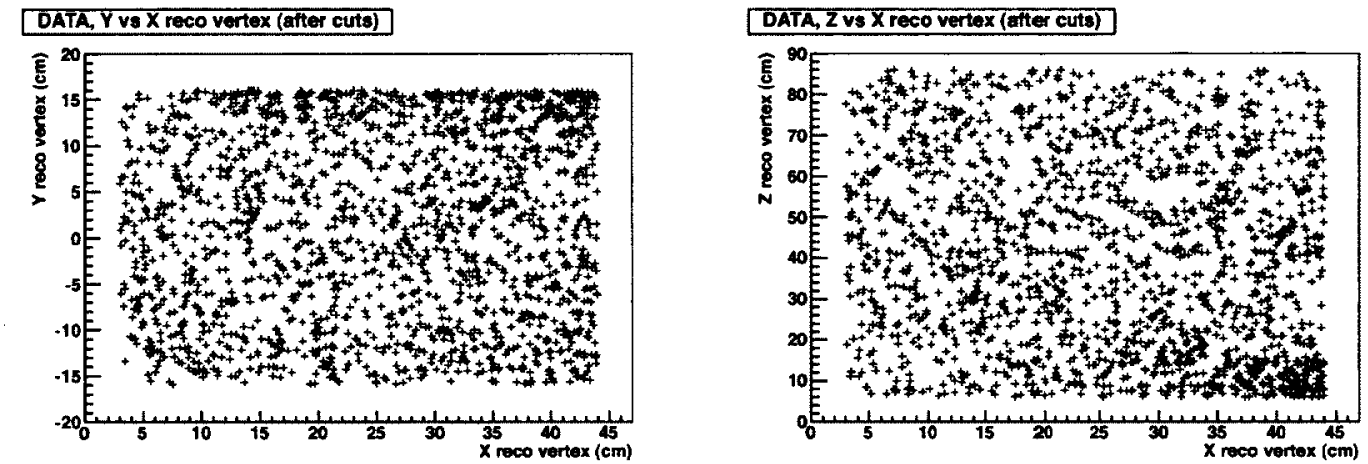

Figure 14.7: The distribution of vertices for all data events that pass automated cuts in the $X / Y$ (left) and $\mathrm{X} / \mathrm{Z}$ (right) two dimensional views.

The muon momentum coming from MINOS is an underestimate as a muon track originating inside the TPC loses energy as is travels through the liquid argon, stainless steel cryostat, and the ArgoNeuT bathtub before finally reaching MINOS. The muon's lost energy needs to be accounted for in three regions depicted in Figure 14.9. Calorimetric reconstruction is used for determining energy loss inside the TPC, which is shown as region 1 . The Geant4-based detector simulation is used for region 2, which starts after the muon exits the TPC and before it enters MINOS first plane. The muon momentum in region 3 is obtained directly from MINOS. The final muon momentum is calculated by summing the energy loss in all three regions. The energy loss versus the distance traveled in region 2 is shown in Figure 14.10. Its shape is attributed to the complex nature of region 2. This is expected as this region is very complicated and the energy loss depends not only on the distance traveled but also the angle of a muon. The inner and outer cryostats, present in this region, 

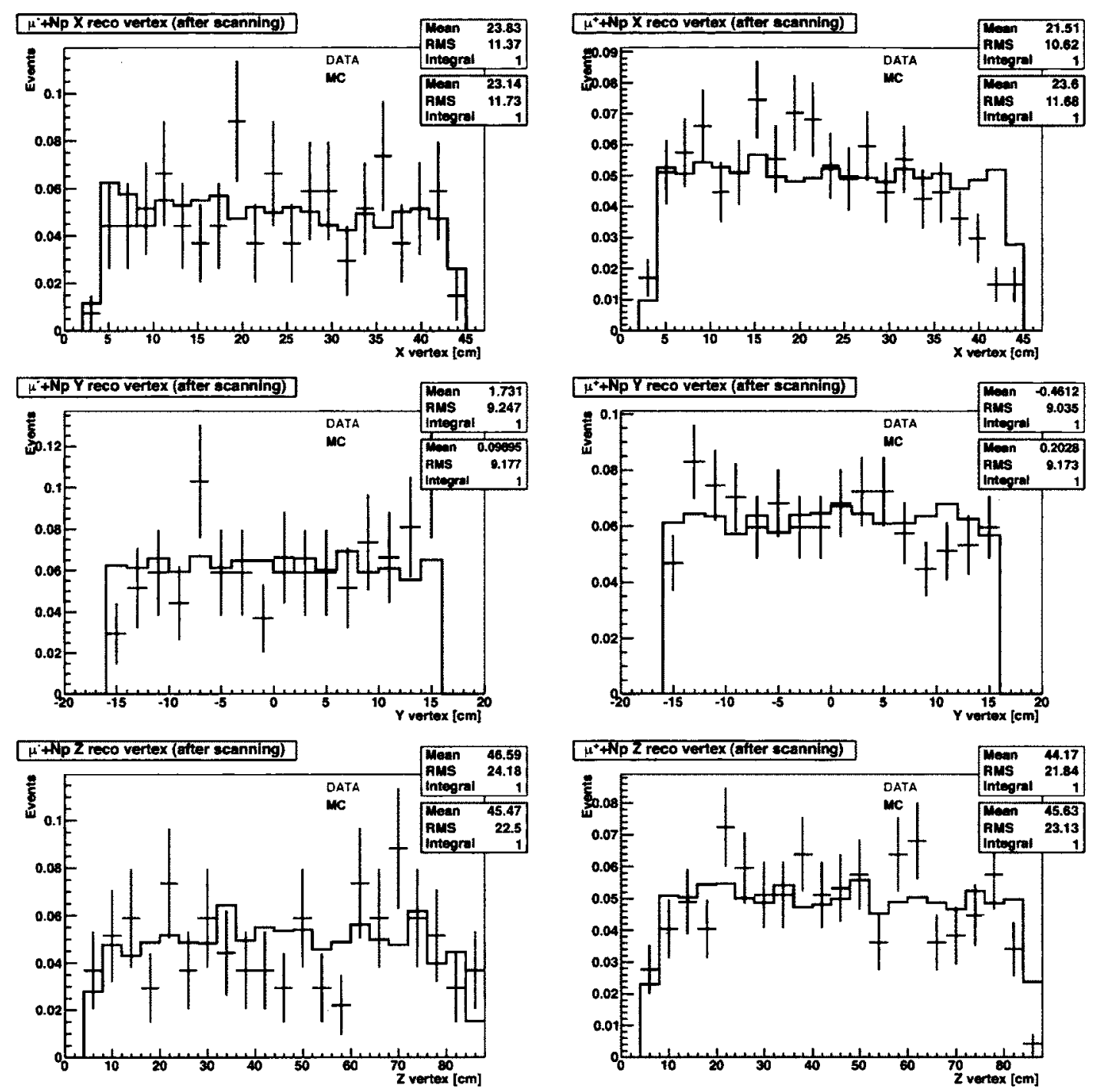

Figure 14.8: The area normalized distribution of vertices for all data events after scanning and comparison with the MC true $\mu+\mathrm{Np}$ events after automated cuts. The left column shows $\mathrm{X}, \mathrm{Y}$, and $\mathrm{Z}$ distributions for neutrinos and right column for anti-neutrinos.

are not uniform in terms of thickness and exact shape as a function of distance. However, a better correlation was found between the distance traveled than the angular dependence. The parameters of the linear fit are used for determining the energy loss of a muon that travels a known distance in the region 2.

There are a couple of matching scenarios, the simplest one being a match between a single ArgoNeuT track and a single muon track in MINOS. There, however, can be more complicated scenarios in which, for example, a single ArgoNeuT track is matched to several 


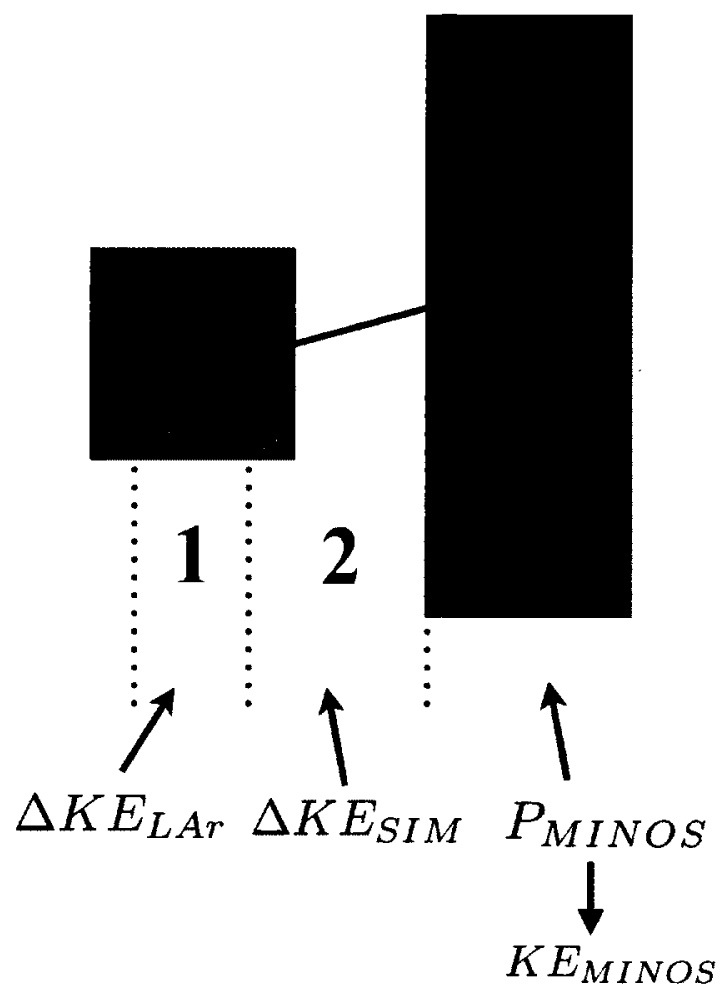

Figure 14.9: Schematic of three regions through which a muon travels. Region 1 is the TPC, region 2 is between the edge of the TPC and MINOS first plane, and region 3 is inside MINOS Near Detector. The energy loss in liquid argon, in region 1, is determined from the calorimetry. The energy loss in region 2 is determined from the Geant 4 simulation and in region 3 directly from MINOS.

tracks in MINOS [84]. In each case the best possible match is picked based on the $\Delta r / \cos \theta$, where $\mathrm{r}$ is the radial difference between the ArgoNeuT track projected onto MINOS and the MINOS reconstructed vertex and $\theta$ is the angle between the MINOS and ArgoNeuT track. The cuts on those variables are set in order to reduce the chance of an ArgoNeuT track to be matched to an incorrect track in MINOS and they have been set based on the Monte Carlo expectation. The angle $\theta$ between the two candidate tracks is required to be less than $0.4 \mathrm{rad}$ and the difference between the radial position of those tracks is required to be less than $27 \mathrm{~cm}$. These cuts are apparent in Figure 14.13, which shows the difference between the projected ArgoNeuT track and the MINOS track in terms of angle and radial position for $\mu+\mathrm{Np}$ events ( $\mu^{-}$on left and $\mu^{+}$on right) passing all the automated cuts. In case of data this refers to events after scanning, and true $\mu+\mathrm{Np}$ after automated cuts in case of MC. Plots are area normalized, errors are statistical only and no background subtraction has been applied to the data at this stage. The spread in $\theta$ and $\mathbf{r}$ is attributed to the vertex 


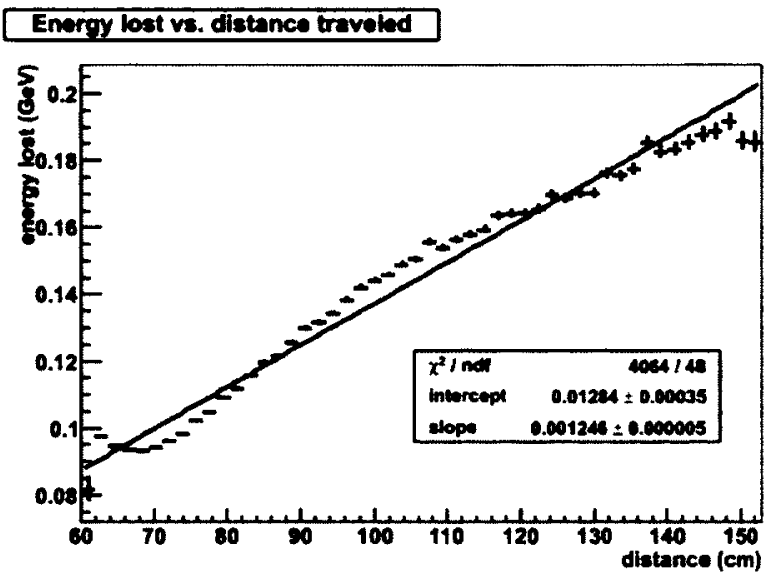

Figure 14.10: The distance between the muon TPC exit point and the point where it reaches the MINOS first plane as a function of energy lost. The shape is attributed to the complicated composition of region 2, consisting of non-uniform thickness and shape of stainless steel parts as a function of distance.

resolution of reconstructed MINOS tracks as well as the determination of exit position and angular resolution for tracks in ArgoNeuT. The imposed cuts on $\mathrm{r}$ and $\theta$ are clearly seen. The distance in $\mathrm{X}$ and $\mathrm{Y}$ between matched MINOS track and the projected ArgoNeuT track is shown in Figure 14.14. Events entering here are data events after scanning and true $\mu+\mathrm{Np}$ events from MC after automated cuts. The MC simulation and data agree to within $1 \mathrm{~cm}$. A much wider distribution is seen for $\mathrm{Y}$ than for $\mathrm{X}$ due to a much better known drift coordinate information. The angles between the exiting ArgoNeuT track and MINOS track is shown in Figure 14.15. The $\mathrm{X}$ angle features a shift and a more narrower distribution for the reasons explained previously. Besides the absolute normalization, the MC models the data quite well. The agreement is slightly better for neutrinos than for anti-neutrinos due to the more energetic muon tracks that are affected less by Coulomb scattering and more likely to enter MINOS.

Besides the geometrical matching requirements, there is also a requirement on the muon charge as reconstructed by MINOS. For neutrinos a match with a negative track in MND is required, and a positive one for anti-neutrinos. However, this cut takes place after the geometrical matching is completed.

After fiducial volume cut and MINOS matching requirement events must pass three additional cuts related to the number of two-dimensional tracks on induction and collection planes as well as the number of exiting three dimensional tracks. They are: 


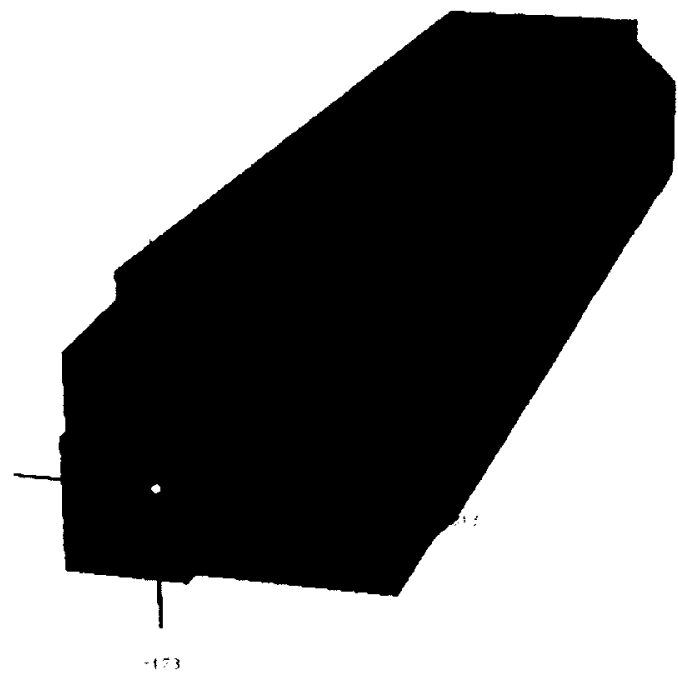

Figure 14.11: Schematic of ArgoNeuT detector (in orange box) in front of MINOS Near Detector. The image shows the relative size and positioning of the two detectors.
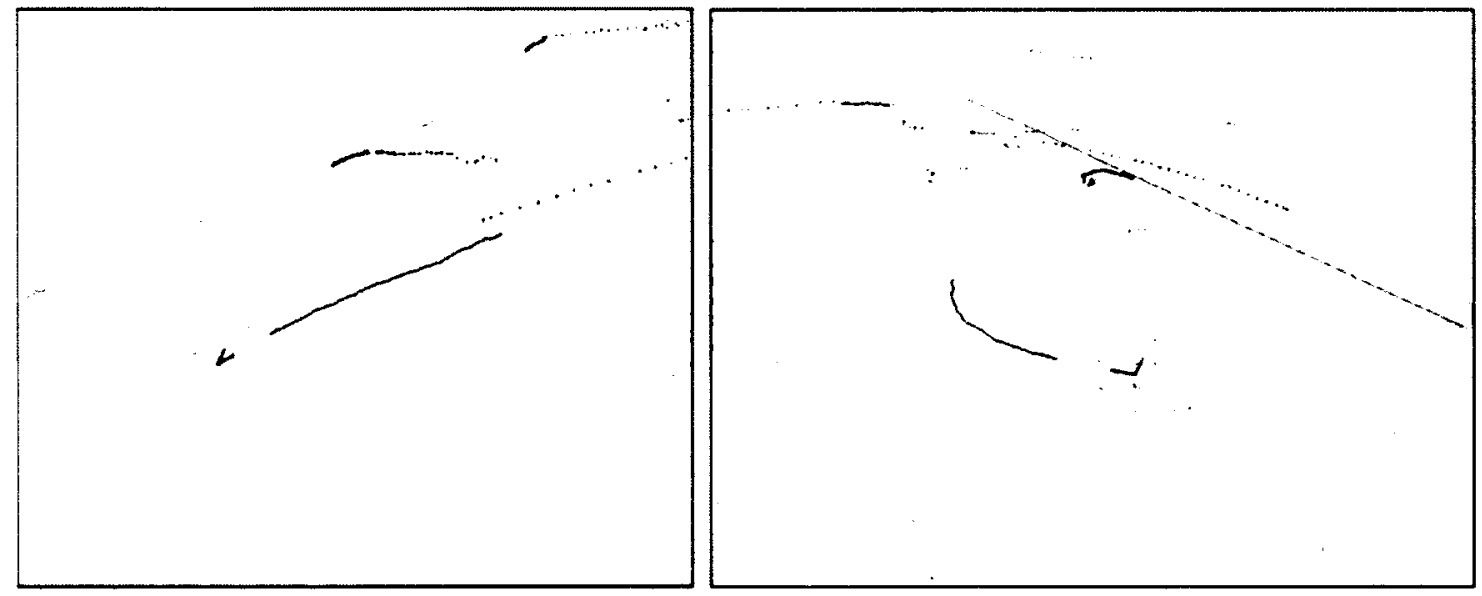

Figure 14.12: Display of the ArgoNeuT and MINOS Near Detector (MND) geometries together with a 3-dimensional tracks reconstructed in the TPC. A through-going track in ArgoNeu'T has been matched to a negatively charged track in the MND. Other tracks present in the MND during the spill are also represented.

1. $1<=$ number of vertex VClusters $<=2$

2. $1<=$ number of vertex LineMergers $<=2$

3. $1<=$ number of exiting $3 \mathrm{~d}$ tracks $<=2$ 

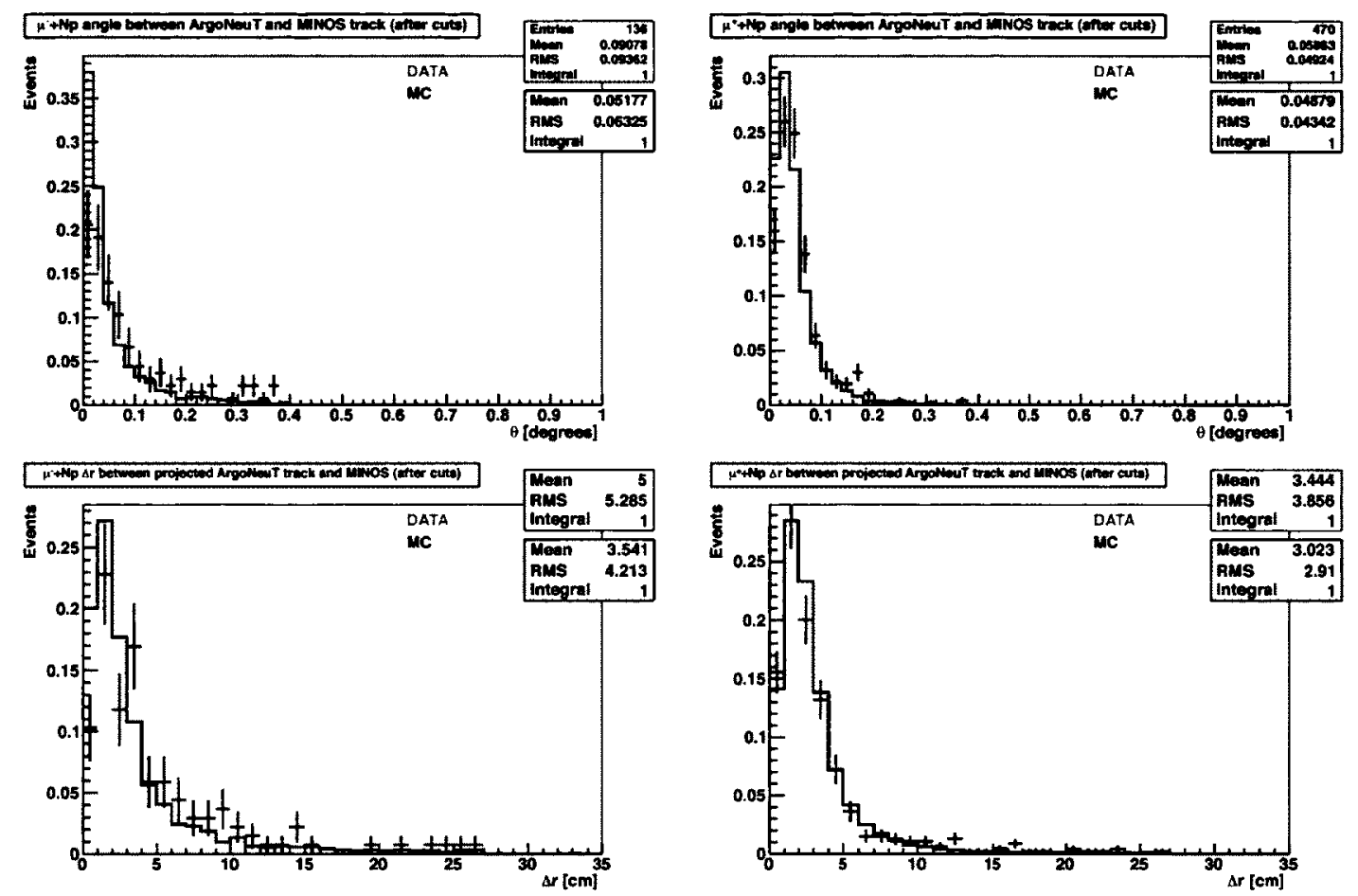

Figure 14.13: Difference between the projected ArgoNeuT track and the MINOS track in terms of angle (top) and radial position (bottom) for $\mu+\mathrm{Np}$ events ( $\mu^{-}$on left and $\mu^{+}$on right) passing all the automated cuts. In case of data this refers to events after scanning, and true $\mu+\mathrm{Np}$ after automated cuts in case of MC. Plots are area normalized, errors are statistical only and no background subtraction has been applied to the data.

In order for a cluster to be considered a 'vertex cluster' its starting position must lie in a rectangle of dimensions 90 ticks by 6 wires centered on a vertex as shown in Figure 14.16.

The distribution of the number of vertex VClusters for $\mu+\mathrm{Np}$ events in the fiducial volume from the Simulation is shown in Figure 14.17 and for the number of vertex LineMergers in Figure 14.18 for neutrinos (left) and anti-neutrinos (right). It is seen that the distributions are very similar between induction and collection planes though not identical. The difference comes from geometrical appearance of an event in two planes. For example, in one view tracks can be overlapping resulting in a fewer number of clusters reconstructed by clustering algorithms. Tracks can also appear much shorter in one view and thus having fewer reconstructed hits, resulting in not forming them as a separate cluster. Due to different appearance of tracks in 2-dimensional views, the cuts on clustering algorithms are imposed in both collection and induction planes separately. The distributions shown in Figure 14.17 are much different for neutrinos where the dominant number of clusters is 1 

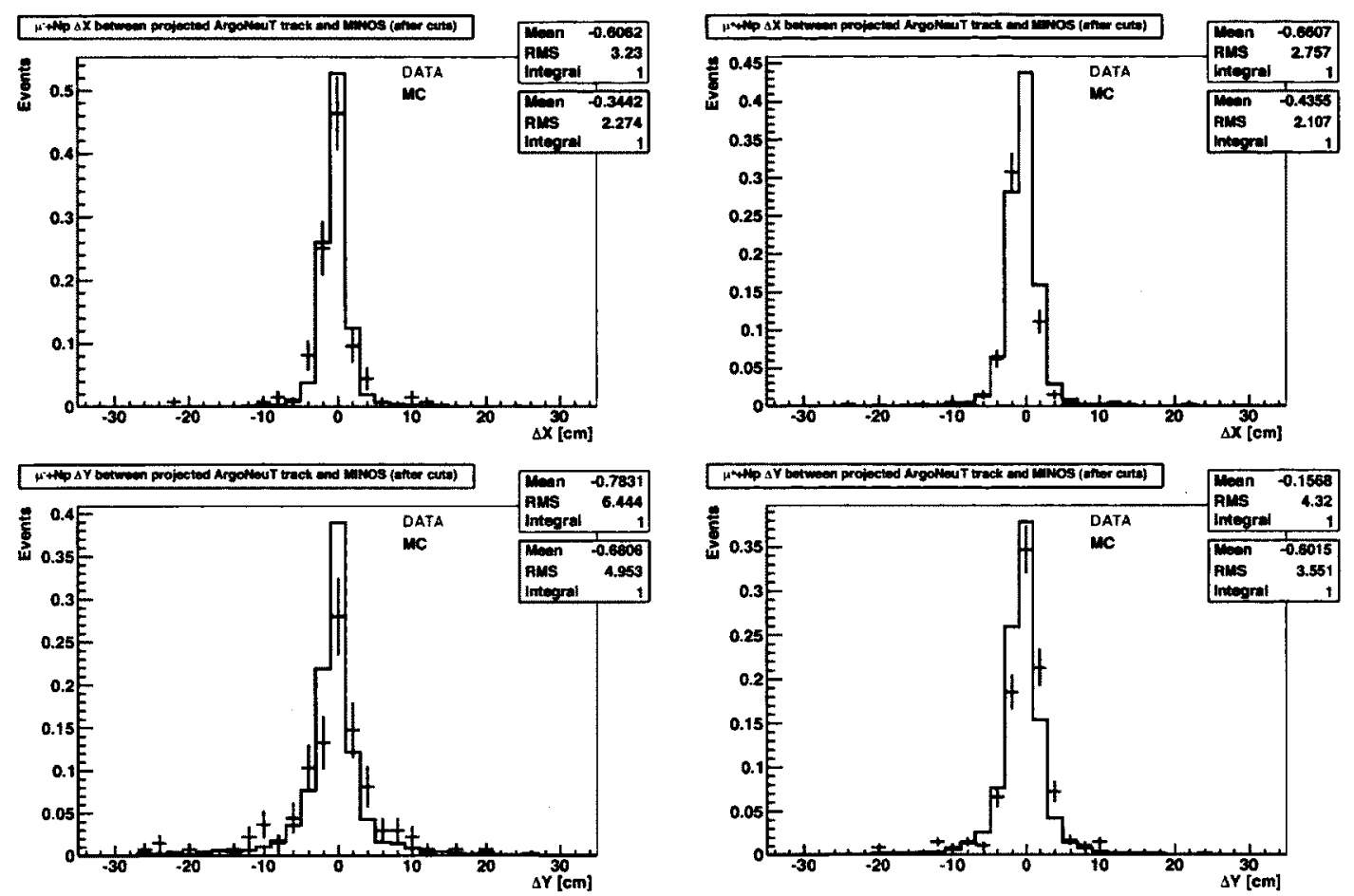

Figure 14.14: The distance in $\mathrm{X}$ (top) and $\mathrm{Y}$ (bottom) between matched MINOS track and the projected ArgoNeuT track for $\mu+N p$ events ( $\mu^{-}$on left and $\mu^{+}$on right) passing all the automated cuts. In case of data this refers to events after scanning, and true $\mu+N p$ after automated cuts in case of MC. Plots are area normalized, errors are statistical only and no background subtraction has been applied to the data.

and 2 than for anti-neutrinos where events mostly feature 1 cluster in each view. This, of course, is expected as most of the events in anti-neutrino mode are much simpler, mostly being $1 \mathrm{mu}+0 \mathrm{p}$. The 0 -th bin is due a failure in clustering. An analogous conclusions can be drawn from the distribution of LineMergers in Figure 14.18. The difference between the distribution of vertex VClusters and vertex LineMergers is the most apparent for neutrinos where there are many more 2-cluster events. This is because VClusters algorithm is much more efficient in forming clusters with fewer number of hits than LineMerger and most $\mu+\mathrm{Np}$ events in neutrinos feature a short track at a vertex. The distribution for the number of exiting 3-dimensional tracks is shown in Figure 14.19 for neutrinos (left) and anti-neutrinos (right). A similar behaviour as in 2-dimensional clustering distribution is seen where there are many more 2 -track events for neutrinos than for anti-neutrinos. The chosen cuts were based on selecting a high number of $\mu+N$ p events while rejecting a significant amount of other events. A more detailed efficiency study of these cuts is described 

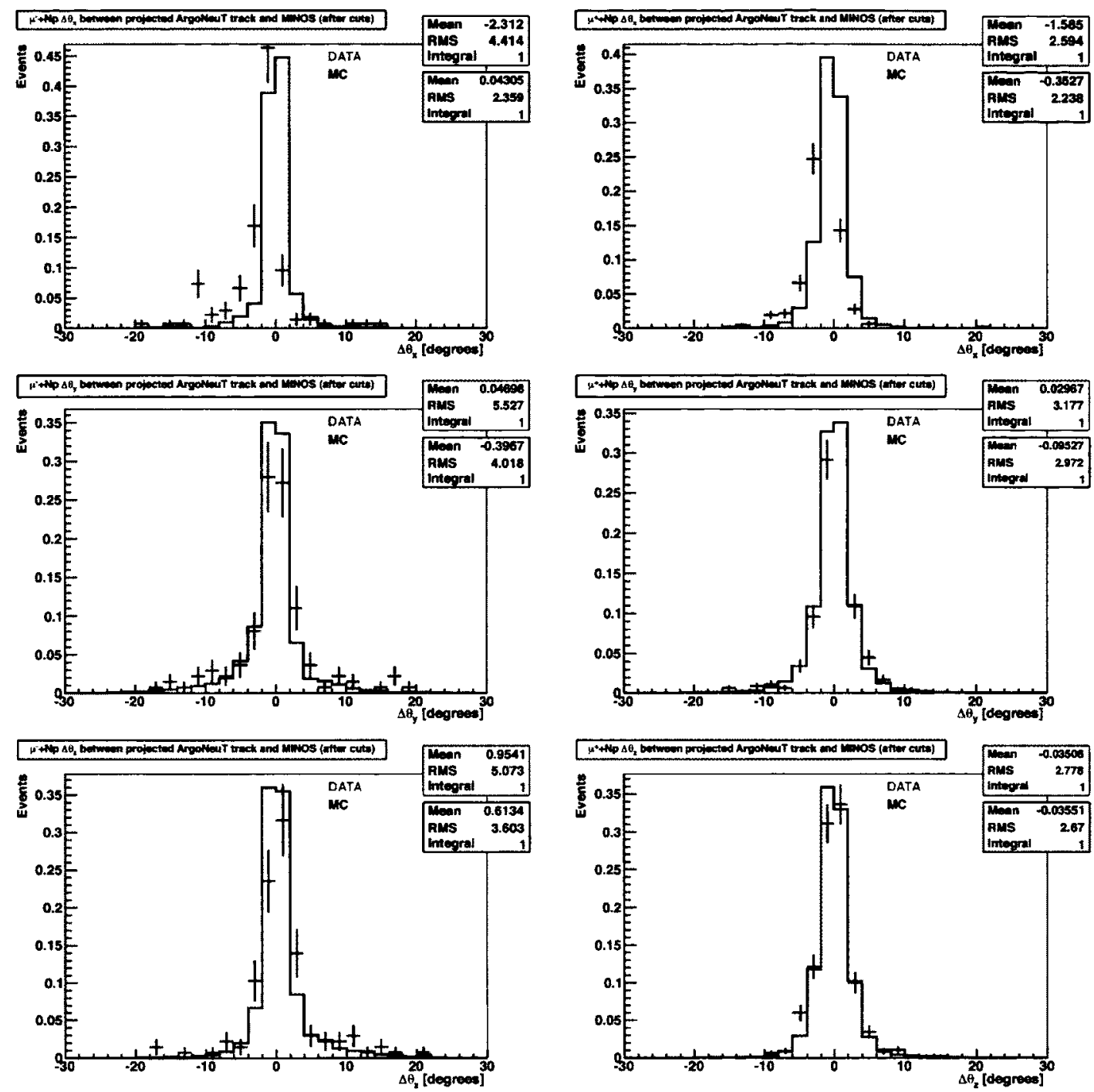

Figure 14.15: The angles between matched MINOS track and the projected ArgoNeuT track for $\mu+\mathrm{Np}$ events ( $\mu^{-}$on left and $\mu^{+}$on right) passing all the automated cuts. In case of data this refers to events after scanning, and true $\mu+\mathrm{Np}$ after automated cuts in case of MC. Plots are area normalized, errors are statistical only and no background subtraction has been applied to the data.

in Section 14.3. The reduction produced by these three cuts on data and MC is shown in Table 14.1 for neutrinos and Table 14.2 for anti-neutrinos. The percentage of additional lost events after each cut is listed, it can be seen that they are very similar on data and MC. A higher number of rejected events in the data for Exiting-Tracks cut is attributed to events featuring a through-going muon/muons in addition to a neutrino interaction, which is not present in the Simulation. Overall, there is a good agreement between data and 


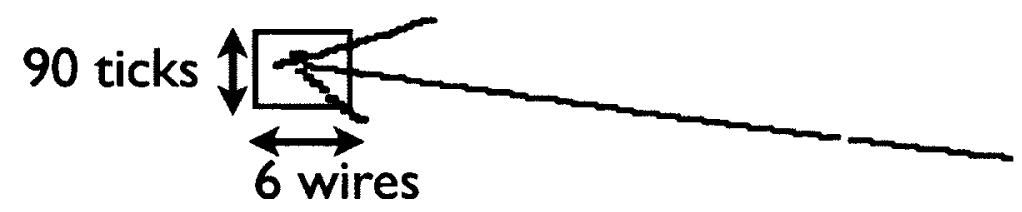

Figure 14.16: A data event showing requirement of 'vertex cluster' being a rectangle centered on a vertex and having dimensions of 90 ticks by 6 wires. In this case, three clusters are counted as 'vertex clusters'.

the $\mathrm{MC}$ for the number of retained events after these three cuts. For neutrinos there are $23.6 \%$ of events in the $\mathrm{MC}$ surviving these cuts and $20.6 \%$ in the data, $47.8 \%$ in the $\mathrm{MC}$ for antineutrinos versus $45.6 \%$ in the data. Again, the slightly higher percentage of retained events in the MC is due to the crossing muons as described above. The area normalized distributions for all three variables for these cuts after matching with MINOS is shown in Figure 14.20 for anti-neutrinos. It is seen that MC and data have a similar shape with the biggest difference for the number of exiting tracks as described earlier. The plots of these three variables are shown after each associated with them cut. The distribution of vertex VClusters after VCluster cut is shown in Figure 14.21. The distribution of vertex LineMergers after LineMerger cut is shown in Figure 14.22. Finally, the distribution of the number of exiting tracks after all automated cuts is shown in Figure 14.23. A good agreement between data and $\mathrm{MC}$ is seen for all of them.

\begin{tabular}{|l|c|c|}
\hline cuts & \% reduction of MC events & \% reduction of data events \\
\hline after VCluster & 58.2 & 61.4 \\
\hline after LineMerger & 27.5 & 25.8 \\
\hline after 3d Exiting Tracks & 22.1 & 27.9 \\
\hline $\begin{array}{l}\text { overall \% of retained } \\
\text { events after above cuts }\end{array}$ & 23.6 & 20.6 \\
\hline
\end{tabular}

Table 14.1: The reduction percentage of events after VCluster, LineMerger and 3d Exiting Tracks cuts for neutrinos in anti-neutrino mode. 
kingaCl on u-plane for $v_{\mu}$

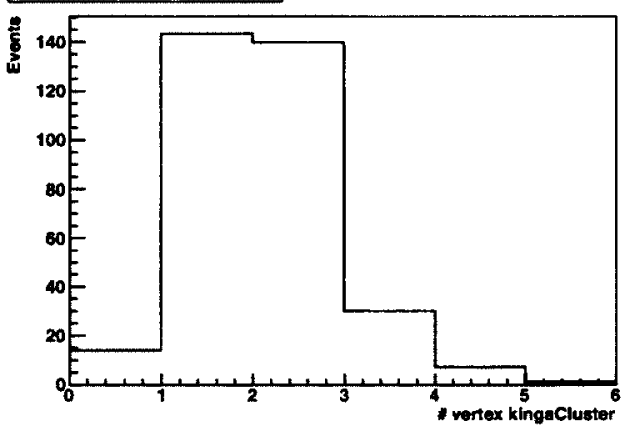

kingaCl on v-plane for $v_{\mu}$

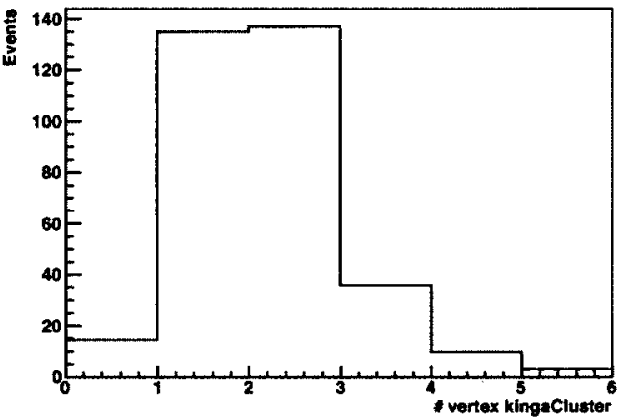

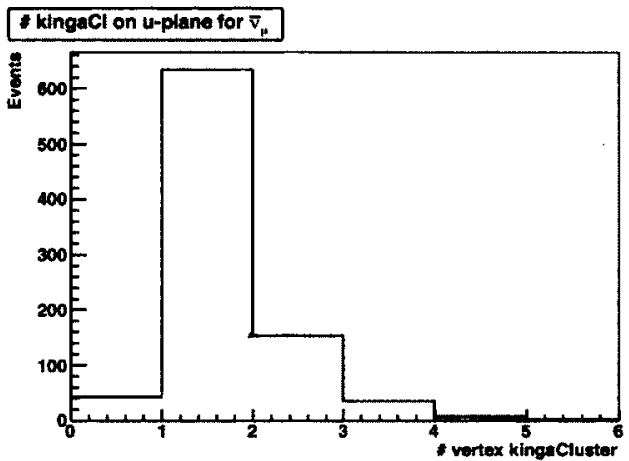

*ingacl on v-plane for $\bar{v}_{4}$

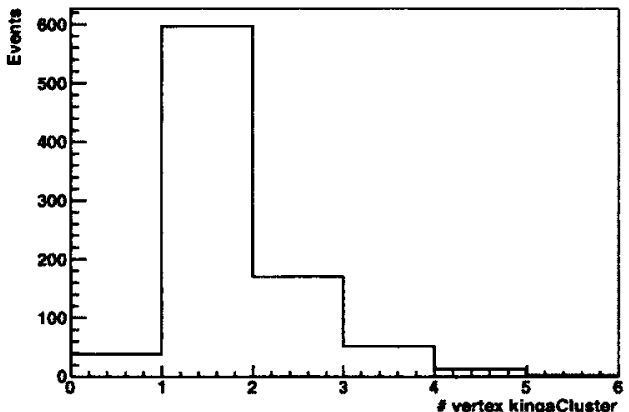

Figure 14.17: Distribution of vertex VClusters on induction(top) and collection (bottom) planes for neutrinos (left) and anti-neutrinos (right) for $m u+\mathrm{Np}$ events in the fiducial volume.

\begin{tabular}{|l|c|c|}
\hline cuts & \% reduction of MC events & \% reduction of data events \\
\hline after VCluster & 37.8 & 38.8 \\
\hline after LineMerger & 17.2 & 14.7 \\
\hline after 3d Exiting Tracks & 7.3 & 12.6 \\
\hline $\begin{array}{l}\text { overall \% of retained } \\
\text { events after above cuts }\end{array}$ & 47.8 & 45.6 \\
\hline
\end{tabular}

Table 14.2: The reduction percentage of events after VCluster, LineMerger and 3d Exiting Tracks cuts for antineutrinos in anti-neutrino mode. 
Lineci on u-plane for $v_{p}$

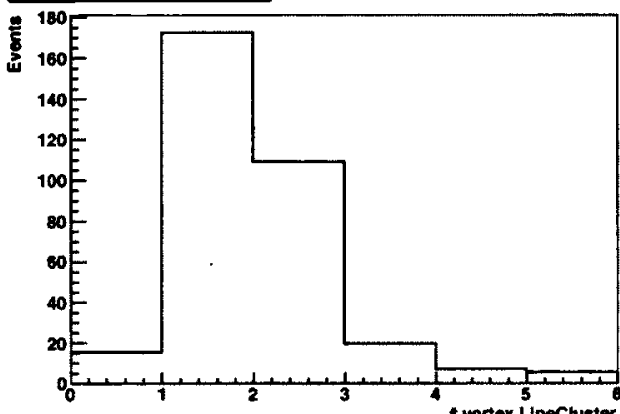

* Lineci on v-plane for $v_{\mu}$

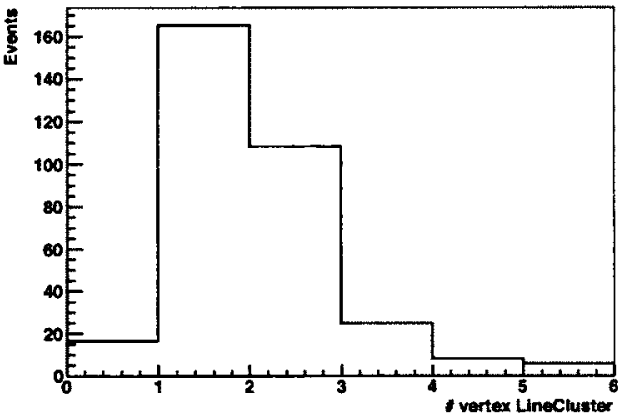

- Uneci on u-plane tor $\nabla_{H}$

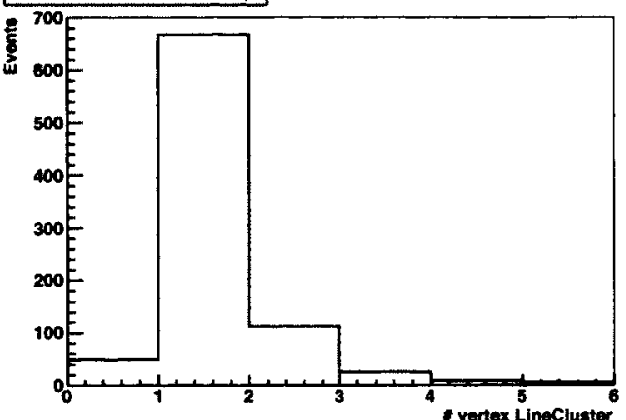

* Linecl on v-plane for $\bar{v}_{u}$

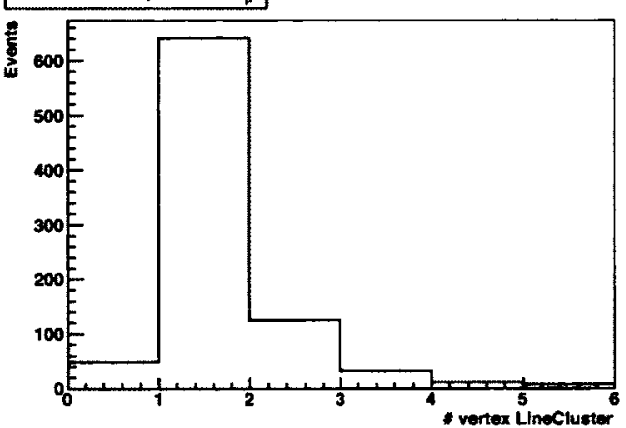

Figure 14.18: Distribution of vertex LineMergers on induction(top) and collection (bottom) planes for neutrinos (left) and anti-neutrinos (right) for $m u+N p$ events in the fiducial volume.

* exting tracks for $v_{\mu}$

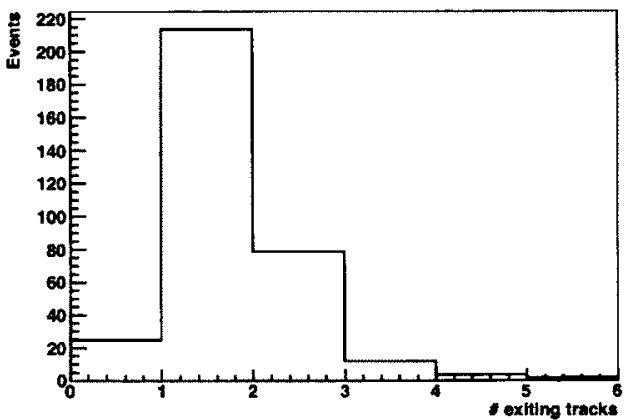

extting tracks for $\bar{v}_{\mathrm{it}}$

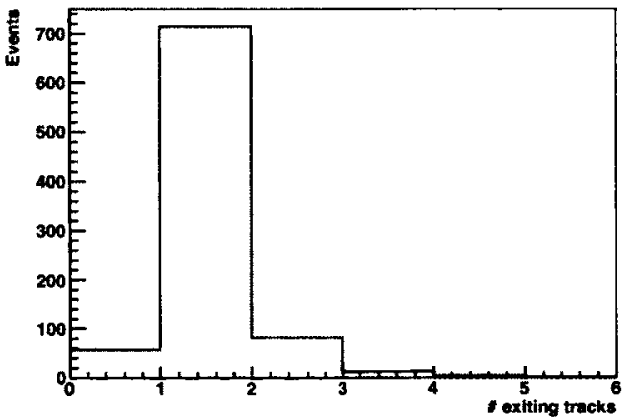

Figure 14.19: Distribution of exiting tracks for neutrinos (left) and anti-neutrinos (right) for $m u+N p$ events in the fiducial volume. 

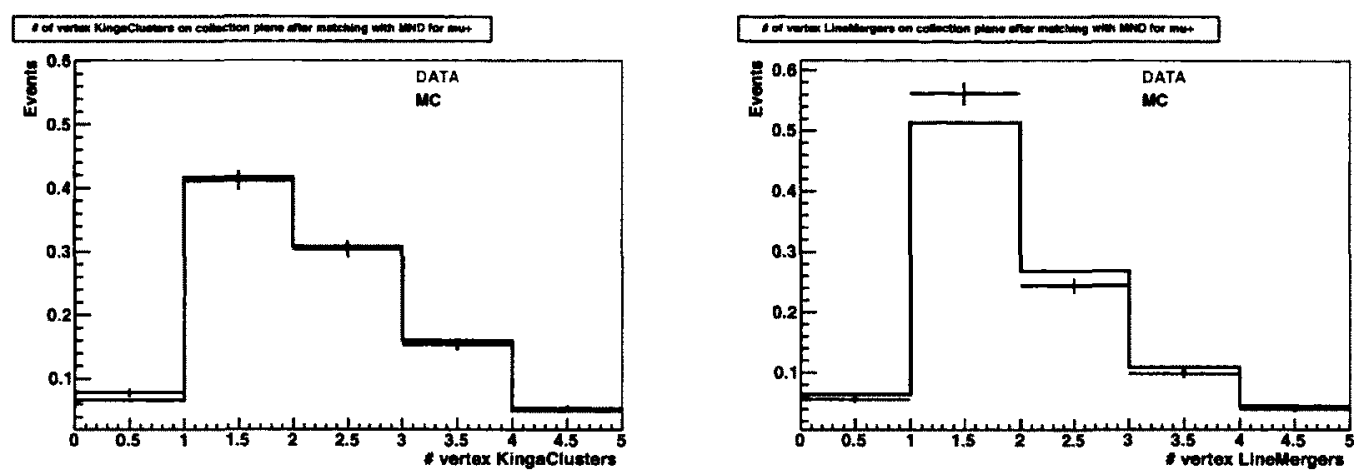

\section{Oof Exting Tracks affer matching with WND for mU+}

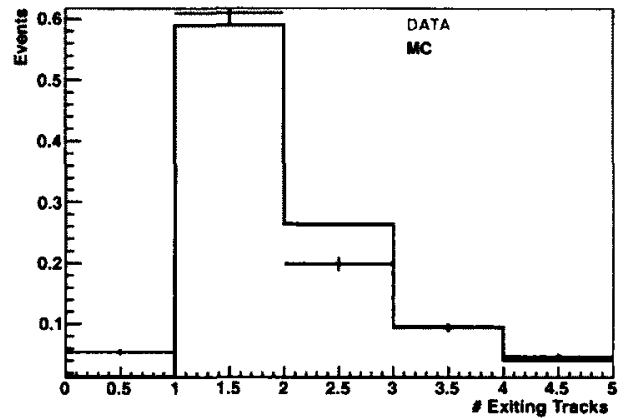

Figure 14.20: The area normalized distributions of number of vertex VCluster (top left), LineMergers (top right) and number of exiting tracks (bottom) after matching with MINOS for anti-neutrinos. Errors are statistical only.

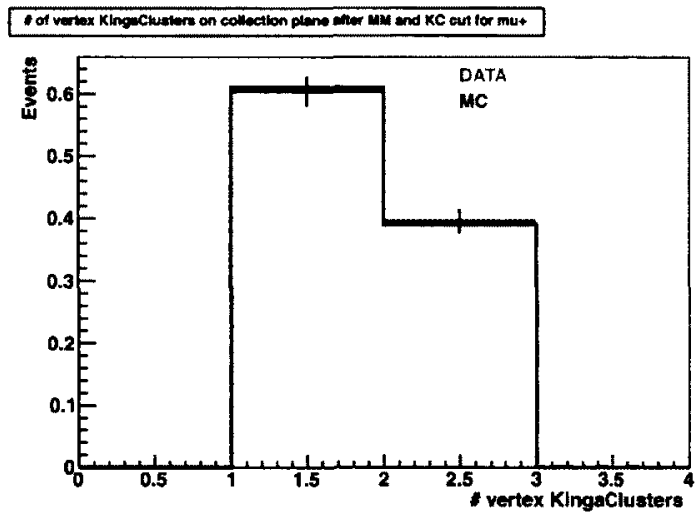

Figure 14.21: Area normalized distribution of number of LineMergers on collection plane for antineutrinos after requiring all the automated cuts with the exception of cut on LineMerger and number of exiting tracks. Errors are statistical only. 


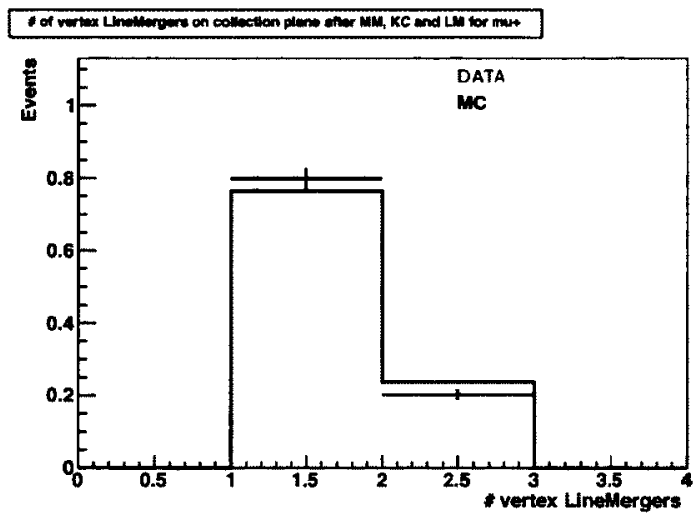

Figure 14.22: Area normalized distribution of number of LineMergers on collection plane for antineutrinos after requiring all the automated cuts with the exception of number of exiting tracks . Errors are statistical only.

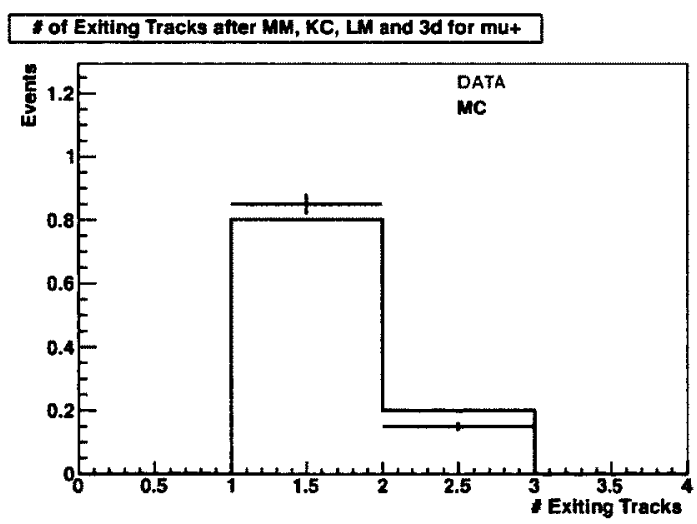

Figure 14.23: Area normalized distribution of number of exiting tracks after all the automated cuts for anti-neutrinos. Errors are statistical only. 


\subsubsection{Proton Reconstruction Threshold}

In order to reconstruct a three-dimensional track a minimum of two hits are required on both the Induction and Collection plane. Since the wire spacing in ArgoNeuT is $4 \mathrm{~mm}$, the shortest track that can be reconstructed corresponds to $4 \mathrm{~mm}$. Looking at the kinetic energy versus length curve for proton in Figure 14.24, it is seen that the track pitch, that is the projection of track length onto wire plane, of $4 \mathrm{~mm}$ corresponds to kinetic energy of about $19 \mathrm{MeV}$.

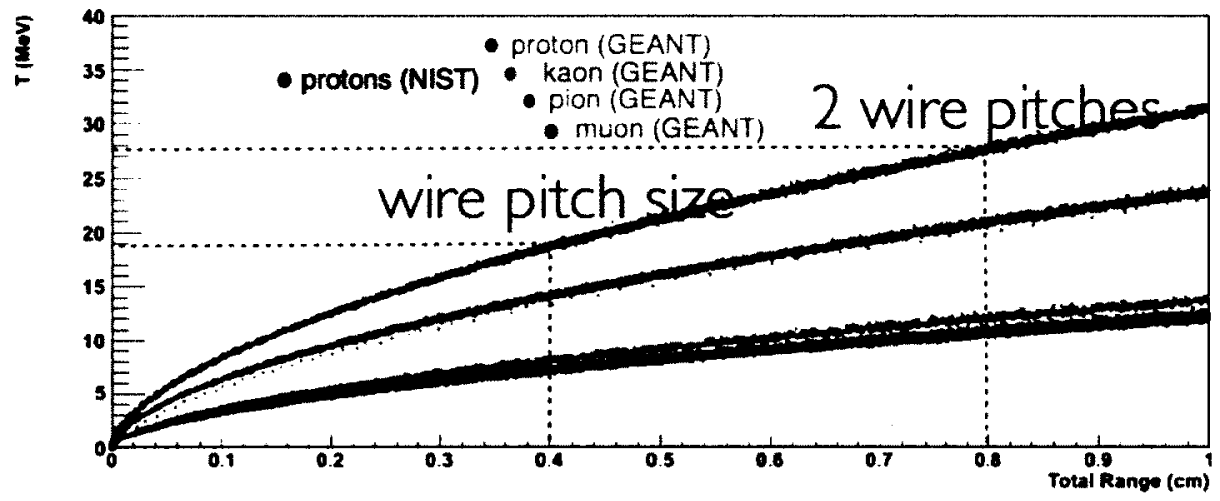

Figure 14.24: Kinetic energy versus length for simulated particles. The minimum wire pitch in ArgoNeuT is also shown and the corresponding to it threshold for protons is indicated.

The threshold is studied further by generating hundreds of MC protons with low momentum, $100 \mathrm{MeV}-300 \mathrm{MeV}$, and examining them on event display. In particular, proton events having just two hits on event display are studied. It is concluded that the lowest sum of the signal coming from the two proton hits corresponds to 170 ADC. The charge in $\mathrm{ADC}$ is converted into the corresponding momentum by the use of $\mathrm{dE} / \mathrm{dx}$ Bethe-Bloch curve for protons and correcting for the recombination effect. The resulting distribution for charge in ADC versus the proton momentum is shown in Figure 14.25. It is seen that the threshold of $170 \mathrm{ADC}$ corresponds to proton momentum of $200 \mathrm{MeV} / \mathrm{c}$.

The proton track can also overlap with the muon track and in this scenario the muon contribution must also be taken into account. Assuming $2.1 \mathrm{MeV} / \mathrm{cm}$ contribution from the muon, $200 \mathrm{ADC}$ are needed for the proton track reconstruction as shown in Figure 14.26. Thus, the ArgoNeuT proton reconstruction threshold is terms of proton kinetic energy is $21 \mathrm{MeV}$. 


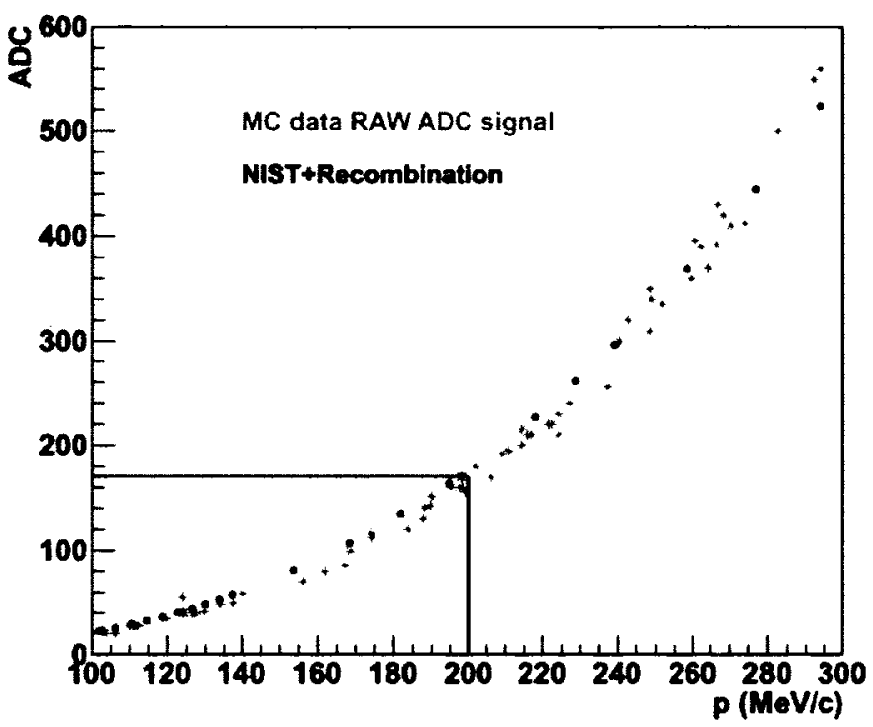

Figure 14.25: Charge in ADC versus proton momentum. The black points correspond to the theory prediction coming from NIST Beta-Bloch equation corrected for recombination effect. The red points come from the ArgoNeuT simulation, where "ADC" refers to the sum of the signal from all proton hits and momentum comes from MC-truth information.

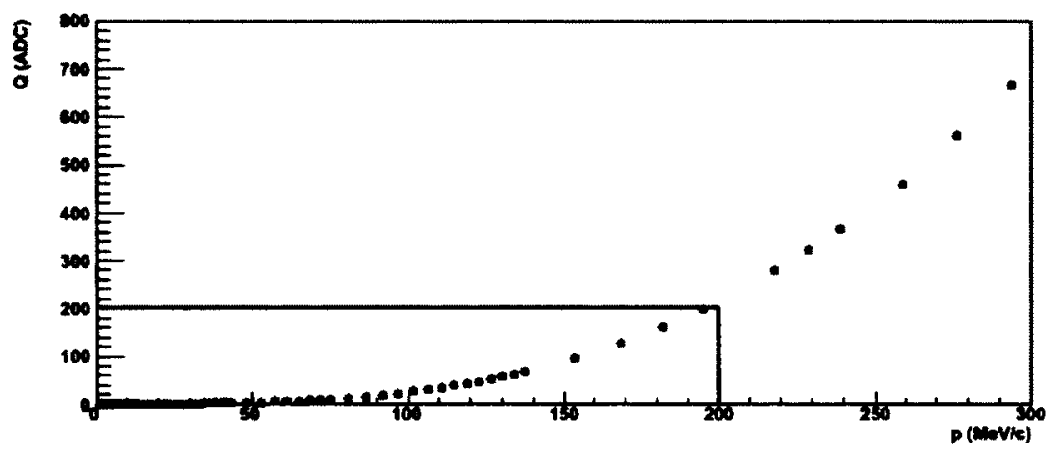

Figure 14.26: Charge in ADC versus proton momentum with the added contribution from the muon.

\subsubsection{Visual Scanning}

Subsamples of data events and MC events selected by the automated cuts are passed to a scanner. The tool used for visual scanning is the analysis display and a recording tool as shown in Figure 14.27. A scanner must decide whether an event is $\mu+\mathrm{Np}$, and thus enters the sample, and record the proton multiplicity at the vertex. Scanner should only pick $\mu+\mathrm{Np}$ events that have all tracks, with the exception of the muon, contained. The 
containment is necessary so that the energy loss as a function of residual range can be performed for particle identification, which is described in the next section. However, at this point in analysis no detailed proton reconstruction is performed. Scanners are asked to look at signal height of each hit belonging to a contained track to make sure their sum is higher than $200 \mathrm{ADC}$ which corresponds to $21 \mathrm{MeV}$ threshold for protons. Only contained tracks above this threshold are counted. A few examples of events that scanner classified as candidate $\mu+\mathrm{Np}$ events are shown in Figures 14.28-14.31. The number of events selected as a function of proton multiplicity are reported in Table 14.3 for neutrinos and Table 14.4 for anti-neutrinos.

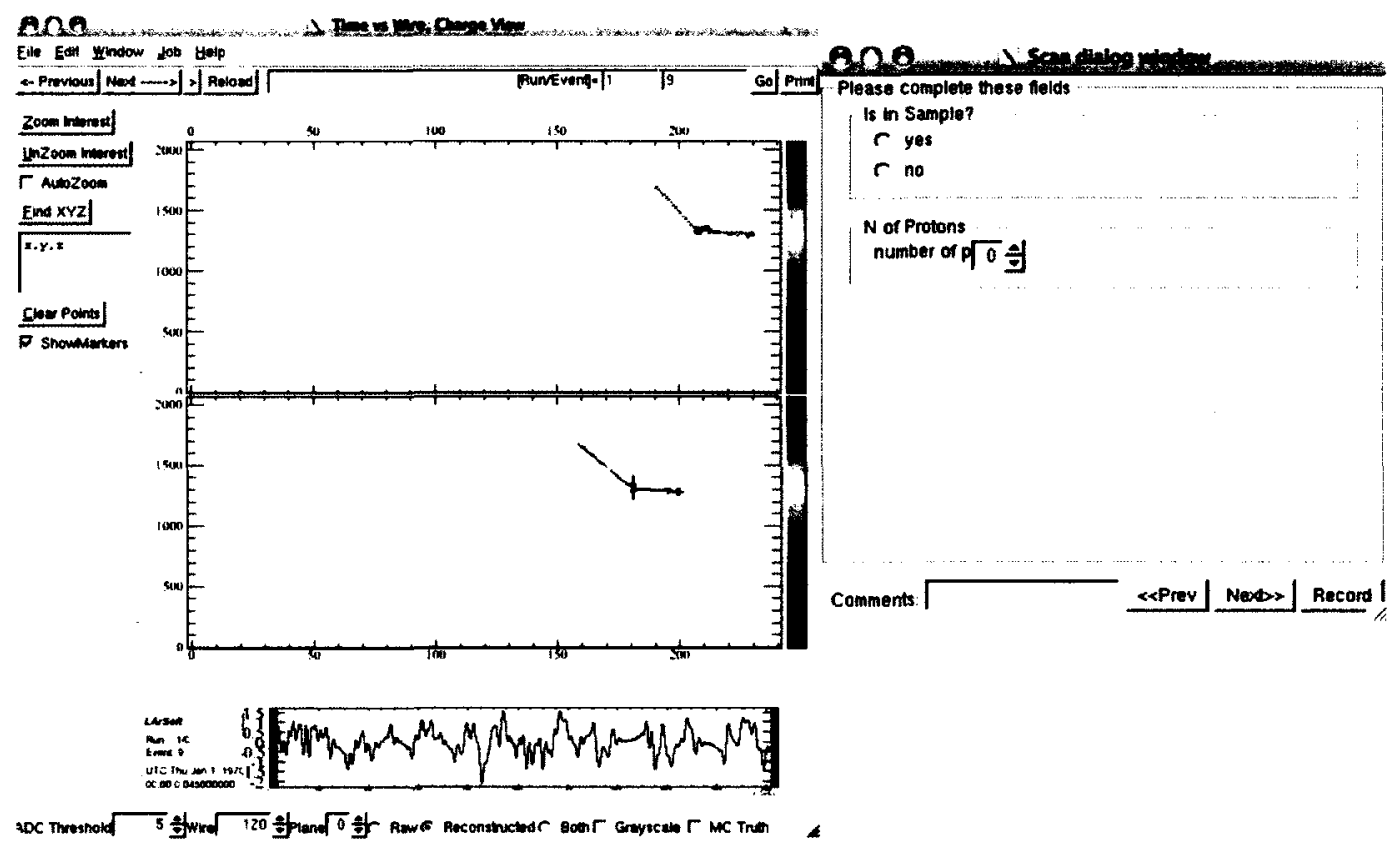

Figure 14.27: Scanning tools 


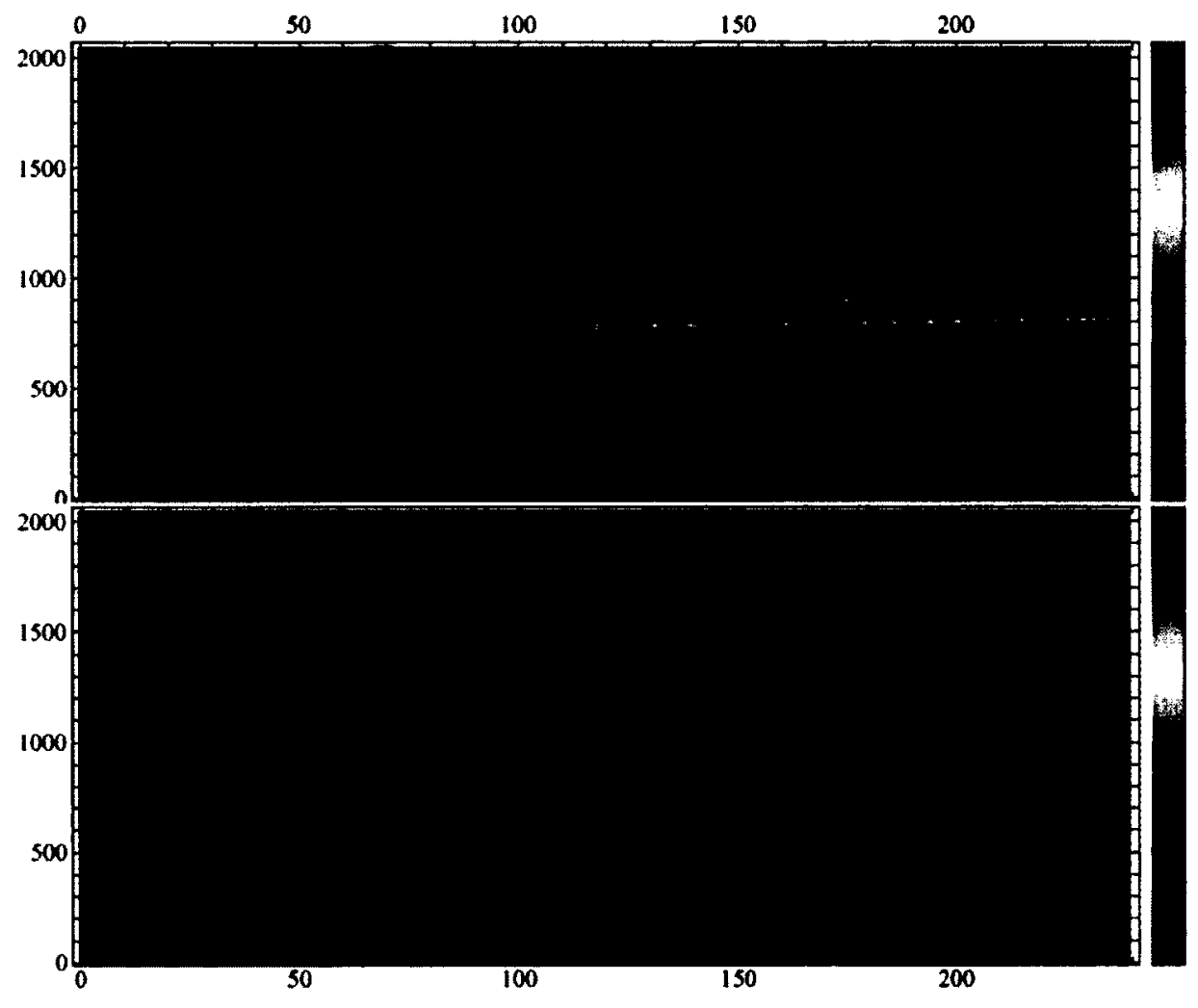

Figure 14.28: Example of candidate $\mu+0 \mathrm{p}$ event 


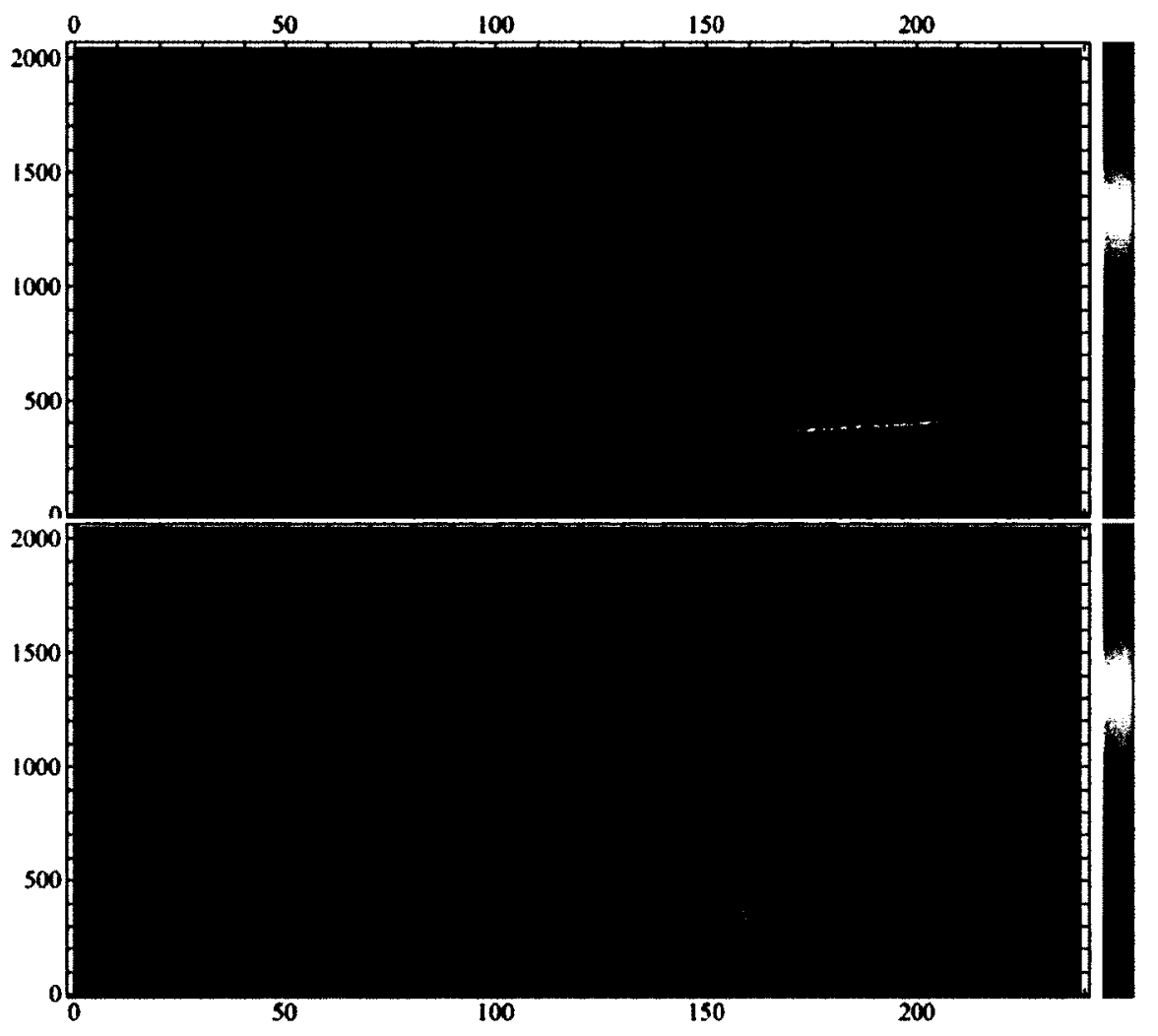

Figure 14.29: Example of candidate $\mu+2 \mathrm{p}$ event 


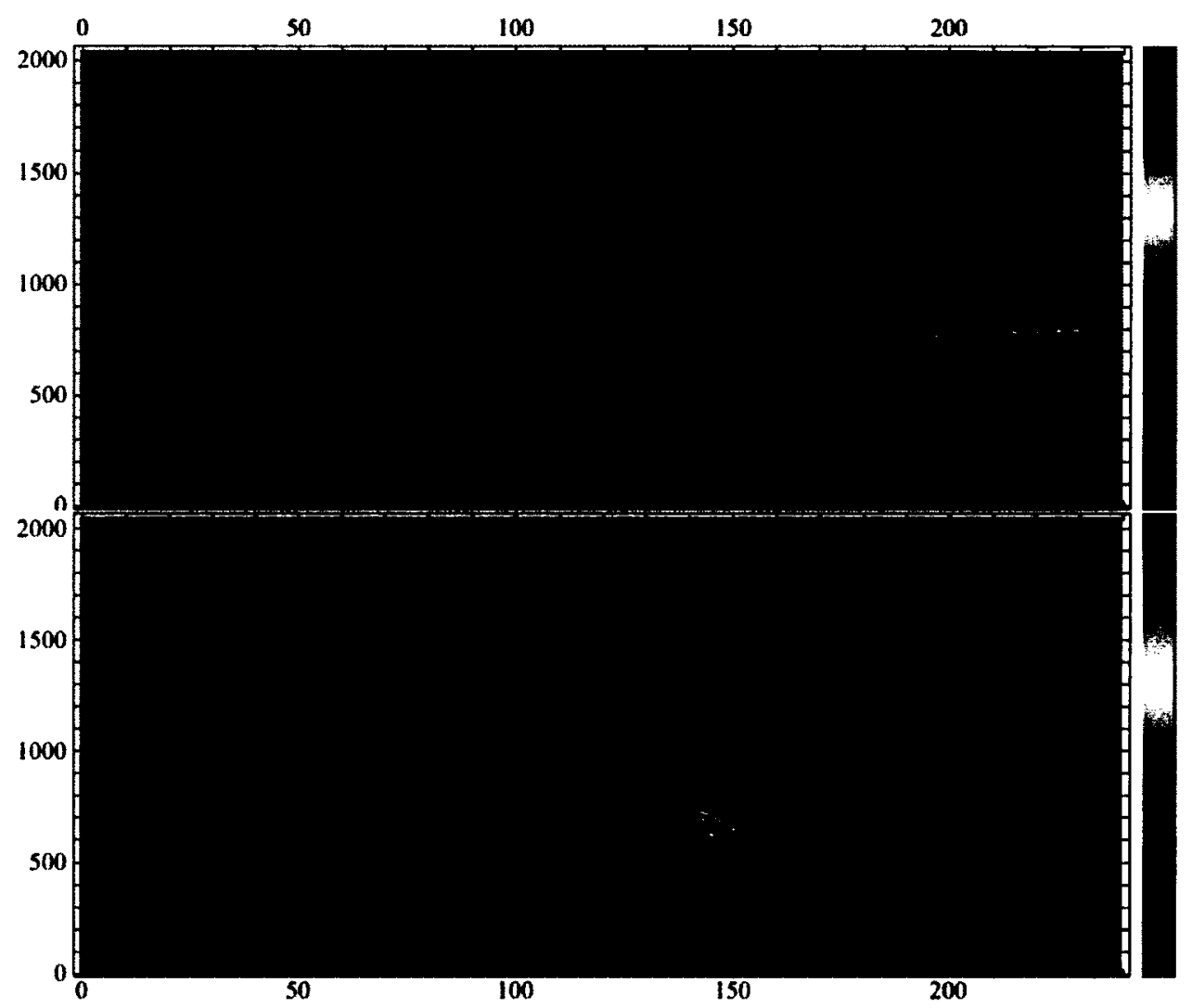

Figure 14.30: Example of candidate $\mu+2 p$ event 


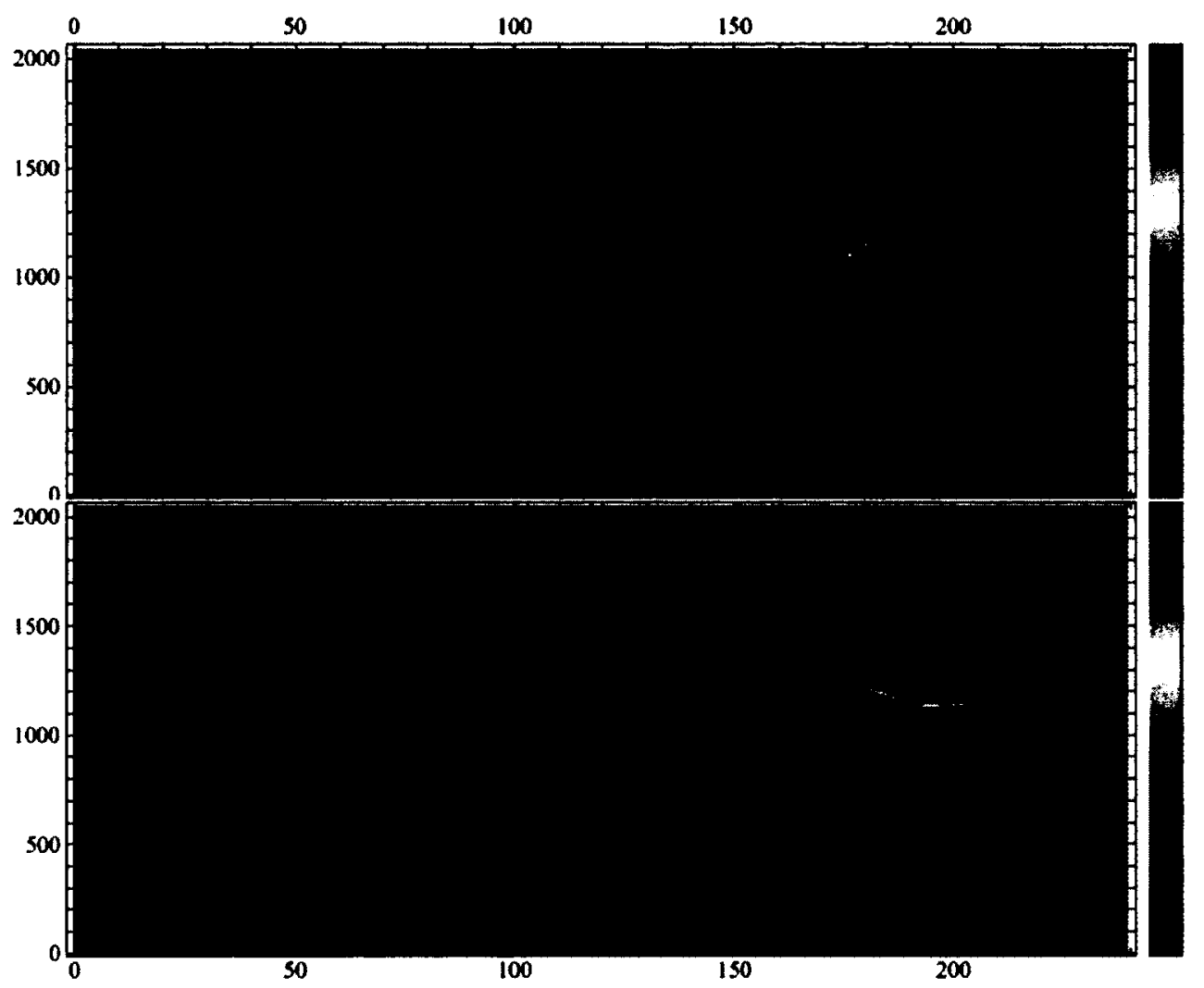

Figure 14.31: Example of candidate $\mu+3 p$ event 
There were $2000 \mathrm{MC}$ events scanned: 1000 neutrinos and 1000 anti-neutrinos in antineutrino mode. From the scanning of this MC sample, calculation of efficiency of scanner for each multiplicity $N, \epsilon_{s c a n}^{N}$, and fraction of true $\mu+N p$ events in each recorded proton bin $M, F_{M}^{N}$, is performed according to Equation 14.3 and Equation 14.4.

$$
\begin{gathered}
\epsilon_{\text {scan }}^{N}=\frac{\text { Number of true } \mu+N \text { pevents in the selected sample }}{\text { Number of true } \mu+N \text { pevents after automated cuts }} \\
F_{M}^{N}=\frac{\text { Number of true } \mu+N p \text { events in marked bin } M}{\text { total number of events in bin } M}
\end{gathered}
$$

The composition of the $\mathrm{MC}$ events after scanning is shown in Figure 14.32 for neutrinos and in Figure 14.33 for anti-neutrinos by using the MC-truth information. "WS" label stands for Wrong Sign selection, referring to an event with $\mu^{+}$in neutrino sample and $\mu^{-}$ in anti-neutrino sample. It can be seen that $\mu+N p$ events both with contained and exiting protons make up majority of the final sample. The biggest background according to the MC simulation consists of events with pions.

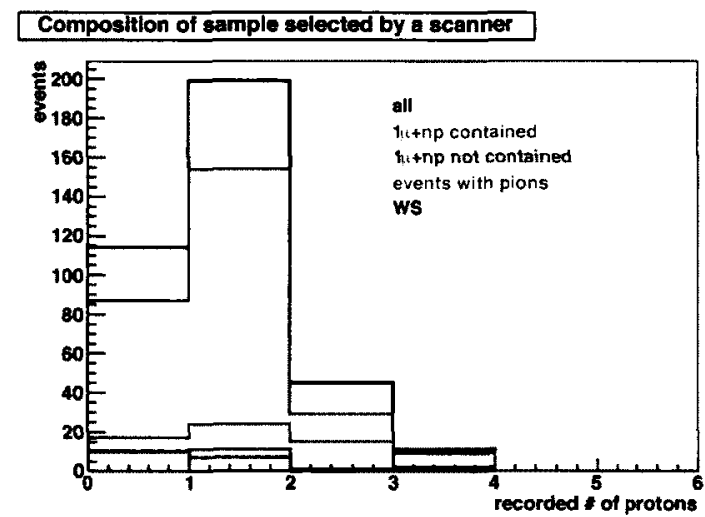

Figure 14.32: Composition of sample picked by a scanner for neutrinos in anti-neutrino mode

\subsubsection{Two Ways of Approach}

At this stage one can perform the analysis of proton multiplicity without using calorimetry. The same automated cuts and visual scanning are performed on data. In order to obtain number of events for multiplicity $\mathrm{N}$ in fiducial volume, $D_{F V}^{N}$, data numbers after scanning 


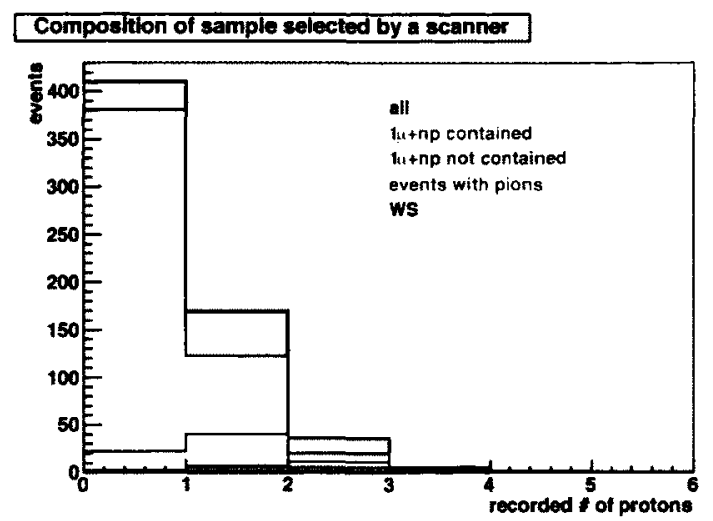

Figure 14.33: Composition of sample picked by a scanner for anti-neutrinos in anti-neutrino mode

for each multiplicity $\mathrm{M}, S_{M}$, should be corrected in the following way:

$$
D_{F V}^{N}=\frac{\sum_{M} S_{M} * F_{M}^{N}}{\epsilon_{c u t s}^{N} * \epsilon_{s c a n}^{N}}
$$

where $\epsilon_{\text {cuts }}^{N}$ is defined according to Equation 14.6.

$$
\epsilon_{\text {cuts }}^{N}=\frac{\text { Number of true } \mu+N p \text { events after automated cuts }}{\text { Number of true } \mu+N \text { pevents in } F V}
$$

This way of approaching analysis will be referred to as 'MC-based method'. Another way of approach is to perform calorimetry for particle identification on the sample picked by the scanner and will be referred to as 'Data-based method'. Calorimetry, in this case, acts as the equivalent of $\mathrm{MC}$ truth, thus there is no need to correct for true fraction of events in each bin selected by a scanner. Instead, to get the number of events in fiducial volume one needs to only correct the number of events for multiplicity $\mathrm{N}$ after calorimetry, $C^{N}$, for efficiency of scanning and cuts as shown in Equation 14.7.

$$
D_{F V}^{N}=\frac{C^{N}}{\epsilon_{c u t s}^{N} * \epsilon_{s c a n}^{N}}
$$

\subsubsection{Calorimetric Reconstruction and Particle Identification}

A powerful method for particle identification can be used for particles that stop in the LArTPC active volume. Charged particles of different mass have different stopping power, which is especially visible as the distance from the track end decreases as shown in Figure 14.34. The shown curves come from a GEANT simulation of stopping protons, kaons, pions and muons. The details of the calorimetric reconstruction are described in Section 
13.8. This method is used on the sample selected by the scanner for particle identification and reconstruction of its kinematics. First, each candidate proton track is reconstructed by manually picking out hits belonging to it and passing them as the input to the threedimensional tracking algorithm described in Section 13.7. This is necessary as currently there is no fully automated reconstruction that is able to do it with a high efficiency. It is a very lengthy process, requiring great care of selecting each hit, that is especially important for events having very short tracks. Once the track is reconstructed it can be passed onto calorimetry. A plot of energy loss along the path $\mathrm{dE} / \mathrm{dx}$ versus residual range and/or a plot of total kinetic energy versus total range are used for particle identification. Examples of this method at work are shown in Figures 14.35-14.40 on events that were selected by a scanner as candidate $\mu+\mathrm{Np}$ events. Events with $\mathrm{N}=0$ look like in Figure 14.35 where a single exiting track is visible with no activity at the vertex. For this class of events, one does not need to use calorimetry if there is no activity at the vertex. At this stage of analysis we already know that this track is a muon since it was matched with MINOS. As an example, $\mathrm{dE} / \mathrm{dx}$ versus residual range plot is shown with the track's overlaid hits which lie on top of the muon prediction. The hits do not follow the simulated curve for small values of residual range as this track is not stopping. The slight rise of $\mathrm{dE} / \mathrm{dx}$ along the track seen in the plot is due to delta rays which are visible as more ionizing, red hits in the collection plane. An example of very short proton track at a vertex is shown in Figure 14.36. It was reconstructed with length of $0.6 \mathrm{~cm}$, which shows how powerful liquid argon technology can be. An example of the longest contained proton track in the selected by the scanner sample is shown in Figure 14.37. Figure 14.38 shows a short proton-like track that is reconstructed as a pion. An example of 2 highly ionizing tracks coming from the vertex is shown in Figure 14.39. One track is reconstructed as a proton, the other as a pion. Thus, this event does not enter the $\mu+\mathrm{Np}$ sample after calorimetry.

The efficiency of the calorimetric reconstruction is discussed in Section 14.3. The data numbers after scanning, $S_{M}$, and after calorimetry, $C^{N}$, for neutrinos are shown in Table 14.3 and for anti-neutrinos in Table 14.4. The reason why the number of events after scanning and after calorimetry are different is due to proton-like tracks from scanning not being reconstructed as a proton, for example as in Figure 14.37. In this case, this event does not enter the final sample. Another reason is that, for example, an event was selected by a scanner as an $\mathrm{N}$-multiplicity event but after careful calorimetric reconstruction its multiplicity was determined as $\mathrm{N}+1$. This is especially common for overlapping proton 
tracks.

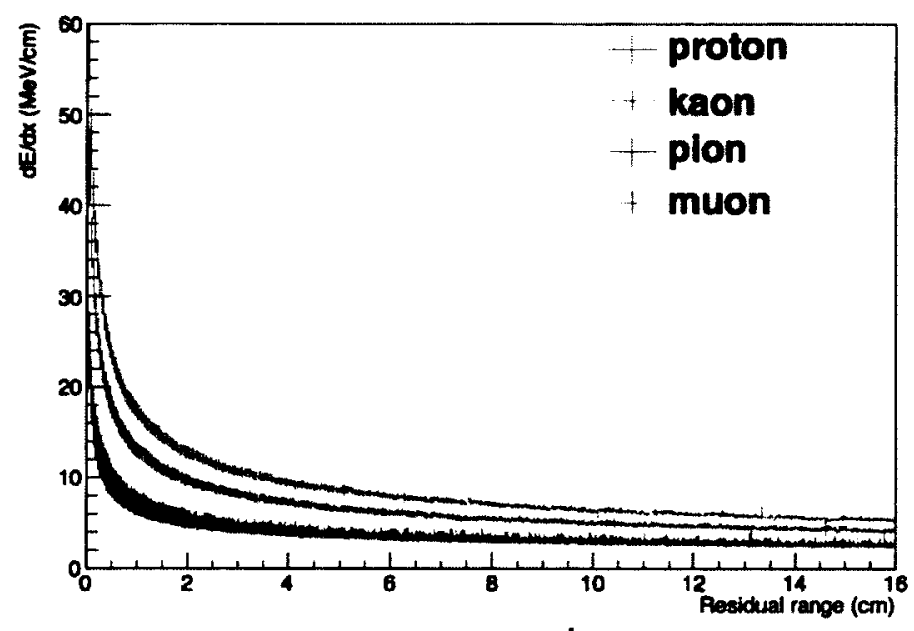

Figure 14.34: Energy loss per unit track length as a function of residual range (distance to the track end) for different simulated charged particles.

\begin{tabular}{|l|c|c|}
\hline Multiplicity & Number of Events after scanning & Number of Events after calorimetry \\
\hline $0 \mathrm{p}+\mu$ & 50 & 50 \\
\hline $1 \mathrm{p}+\mu$ & 66 & 56 \\
\hline $2 \mathrm{p}+\mu$ & 17 & 16 \\
\hline $3 \mathrm{p}+\mu$ & 2 & 3 \\
\hline $4 \mathrm{p}+\mu$ & 1 & 1 \\
\hline Total & 136 & 126 \\
\hline
\end{tabular}

Table 14.3: Number of $\mu+N p$ data events after scanning and calorimetry for neutrinos in antineutrino mode.

\begin{tabular}{|l|c|c|}
\hline Multiplicity & Number of Events after scanning & Number of Events after calorimetry \\
\hline $0 \mathrm{p}+\mu$ & 333 & 333 \\
\hline $1 \mathrm{p}+\mu$ & 115 & 91 \\
\hline $2 \mathrm{p}+\mu$ & 18 & 16 \\
\hline $3 \mathrm{p}+\mu$ & 1 & 2 \\
\hline $4 \mathrm{p}+\mu$ & 3 & 1 \\
\hline Total & 470 & 443 \\
\hline
\end{tabular}

Table 14.4: Number of $\mu+\mathrm{Np}$ data events after scanning and calorimetry for anti-neutrinos in anti-neutrino mode. 

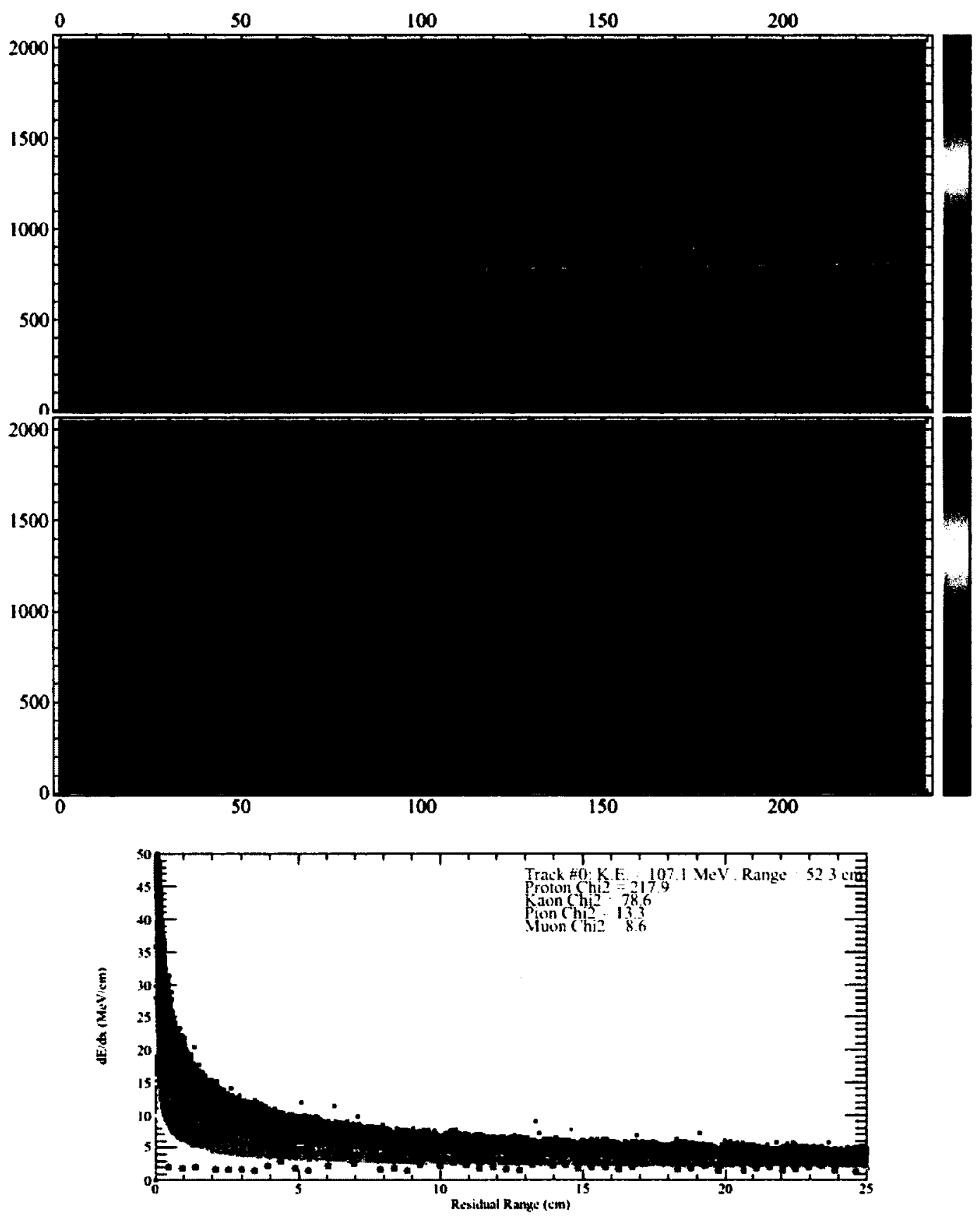

Figure 14.35: Event display showing a single track with no activity at the vertex. $\mathrm{dE} / \mathrm{dx}$ versus residual range plot is shown with the tracks's overlaid hits which lie on top of the muon prediction. The hits do not follow the simulated curve for small values of residual range as this track is not stopping. The slight rise of $\mathrm{dE} / \mathrm{dx}$ along the track seen in the plot is due to delta rays which are visible as more ionizing, red hits in the collection plane. It is categorized as mu+0p event. 

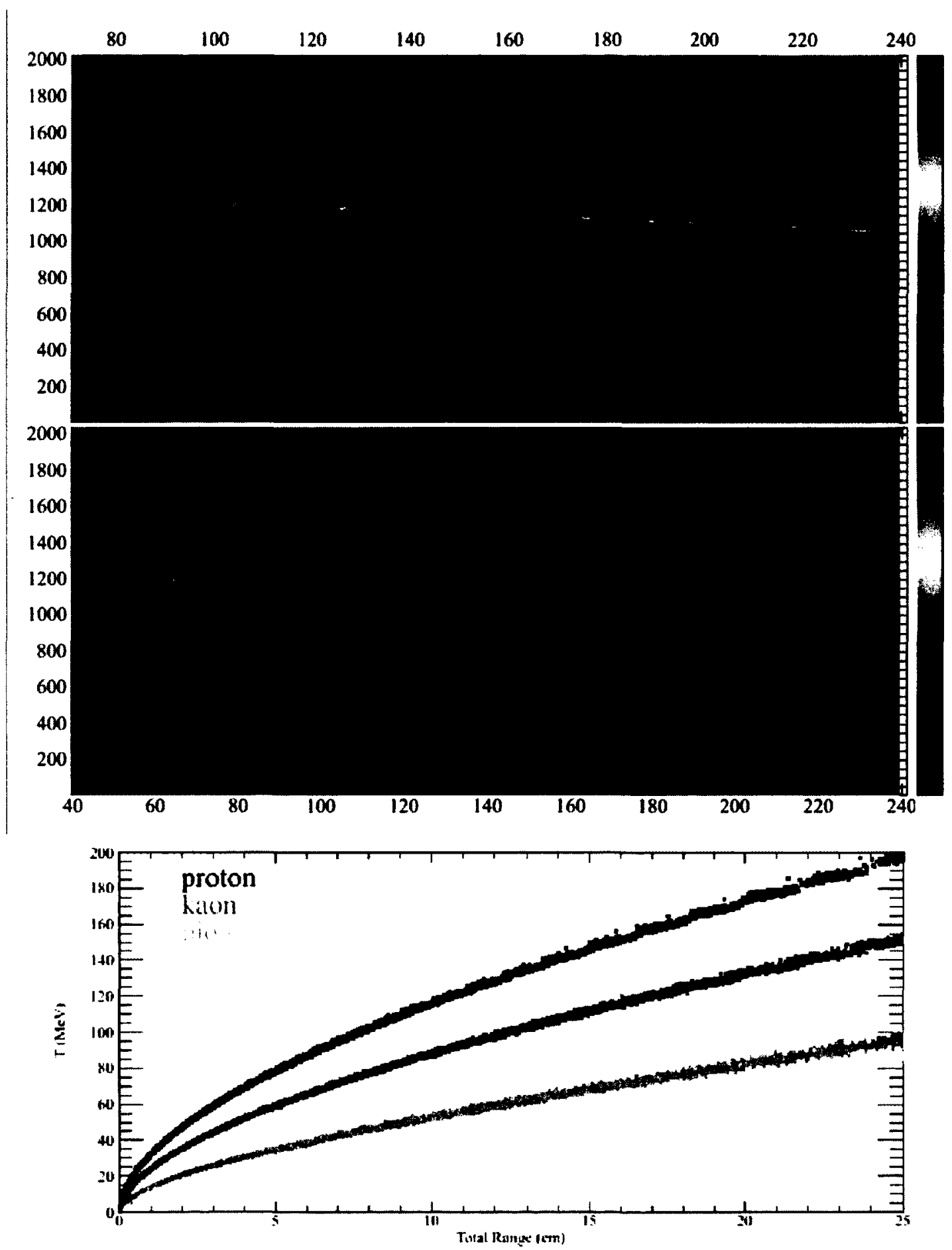

Figure 14.36: Event display showing a single track and high ionization at the vertex which is reconstructed as $0.6 \mathrm{~cm}$ long proton track. 

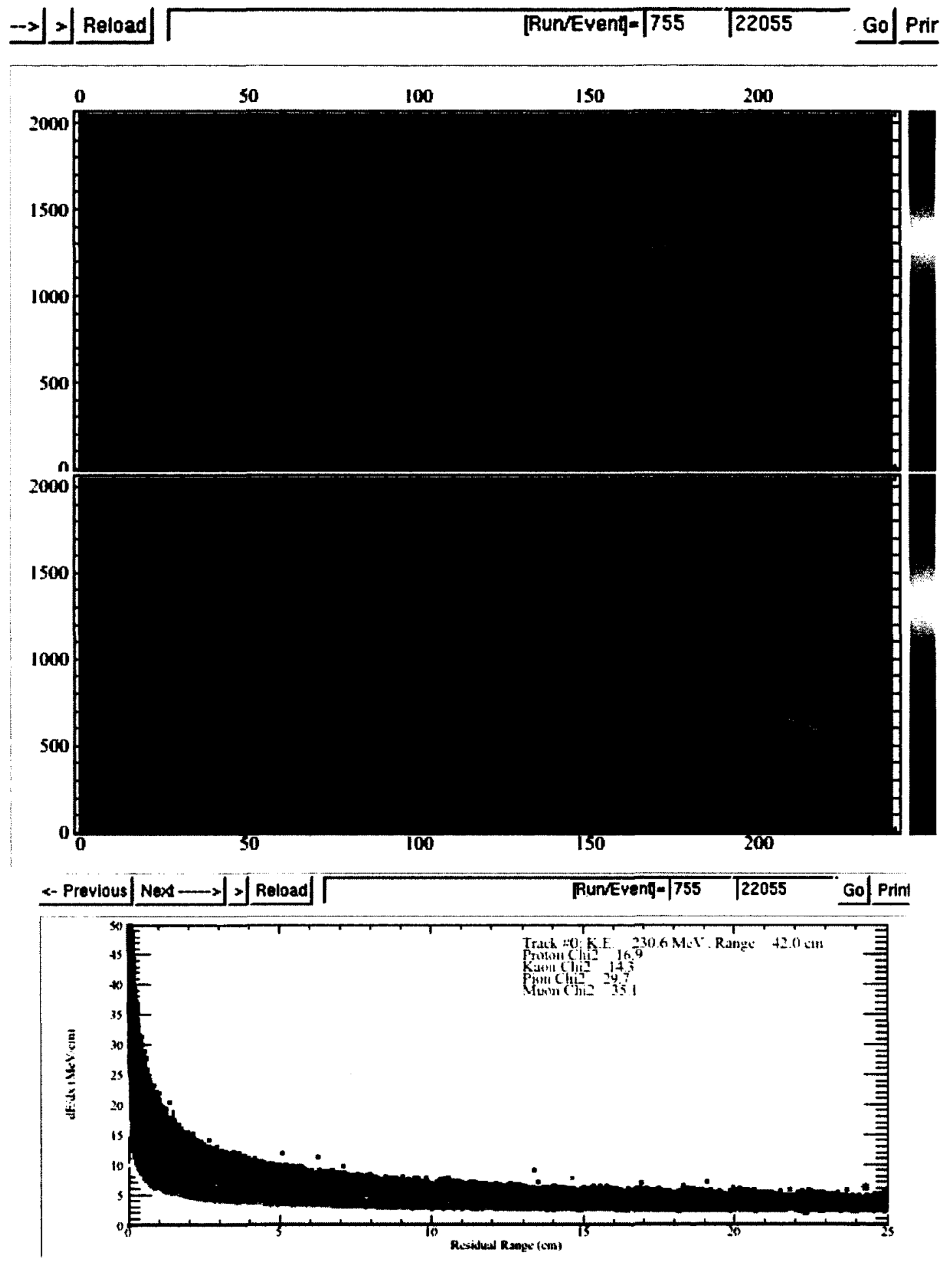

Figure 14.37: Event display showing a muon track with a long proton track. 

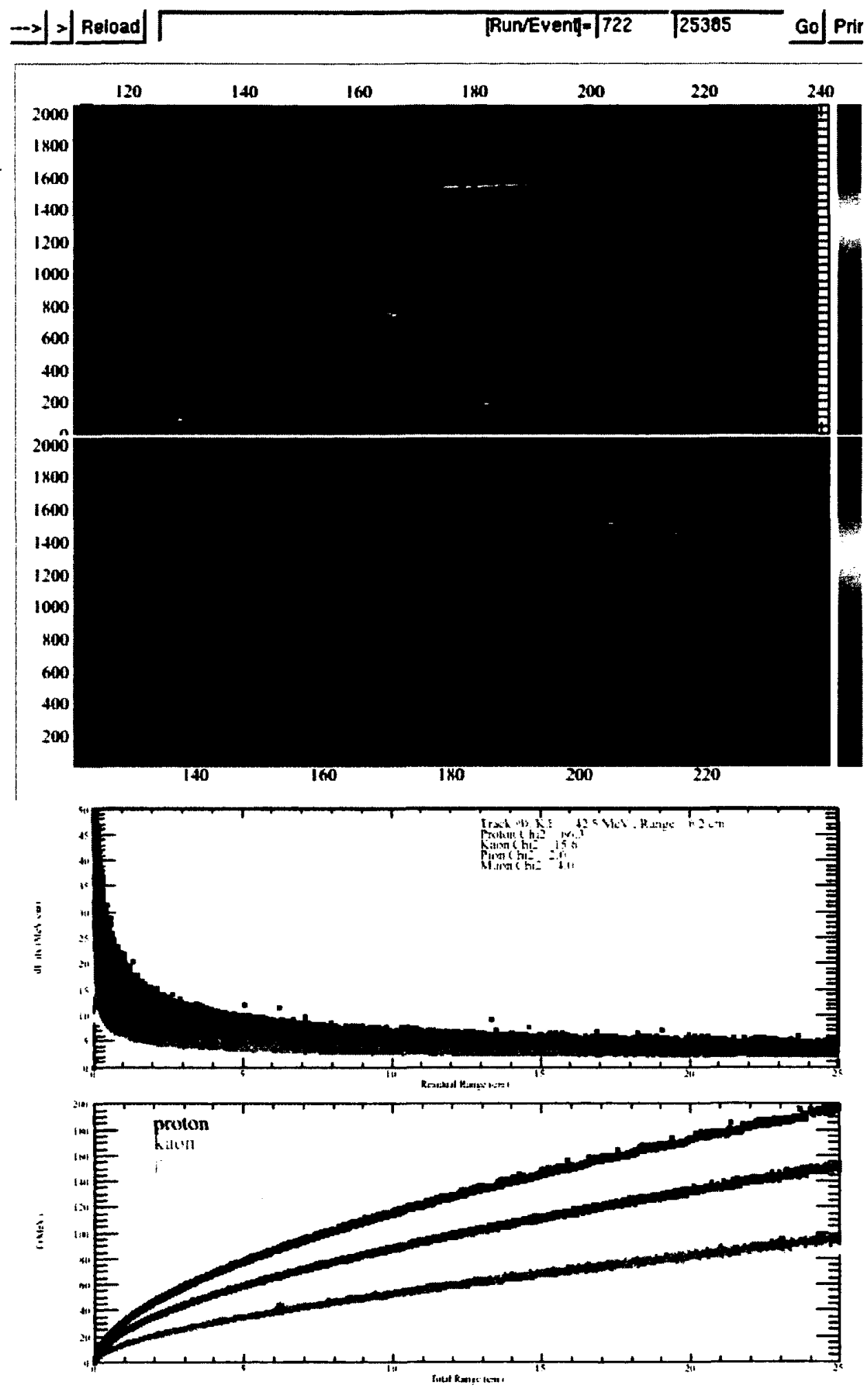

Figure 14.38: Event display showing a muon and a reconstructed pion track. 

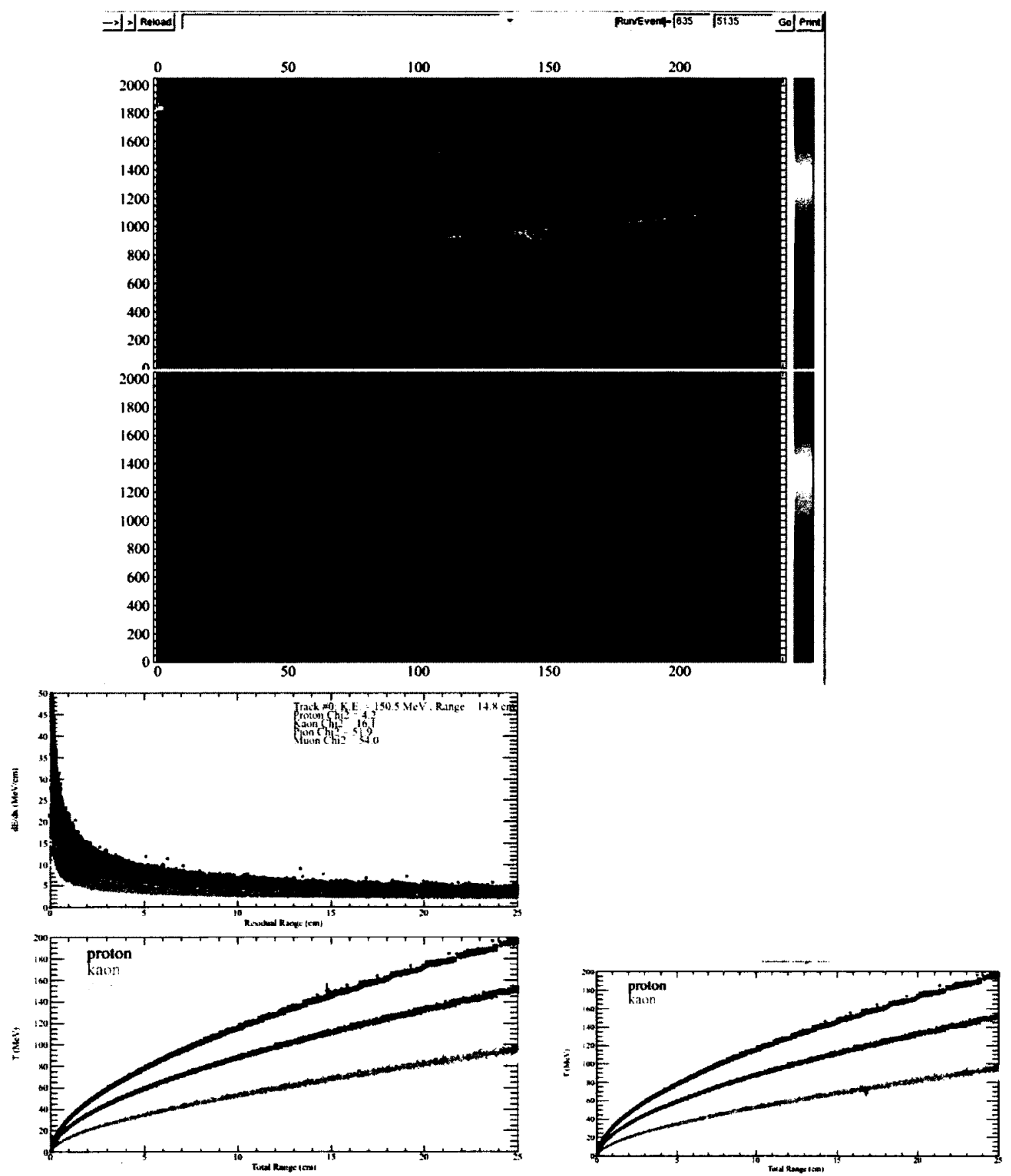

Figure 14.39: Event display showing a muon track accompanied by a proton and a pion track at the vertex . 

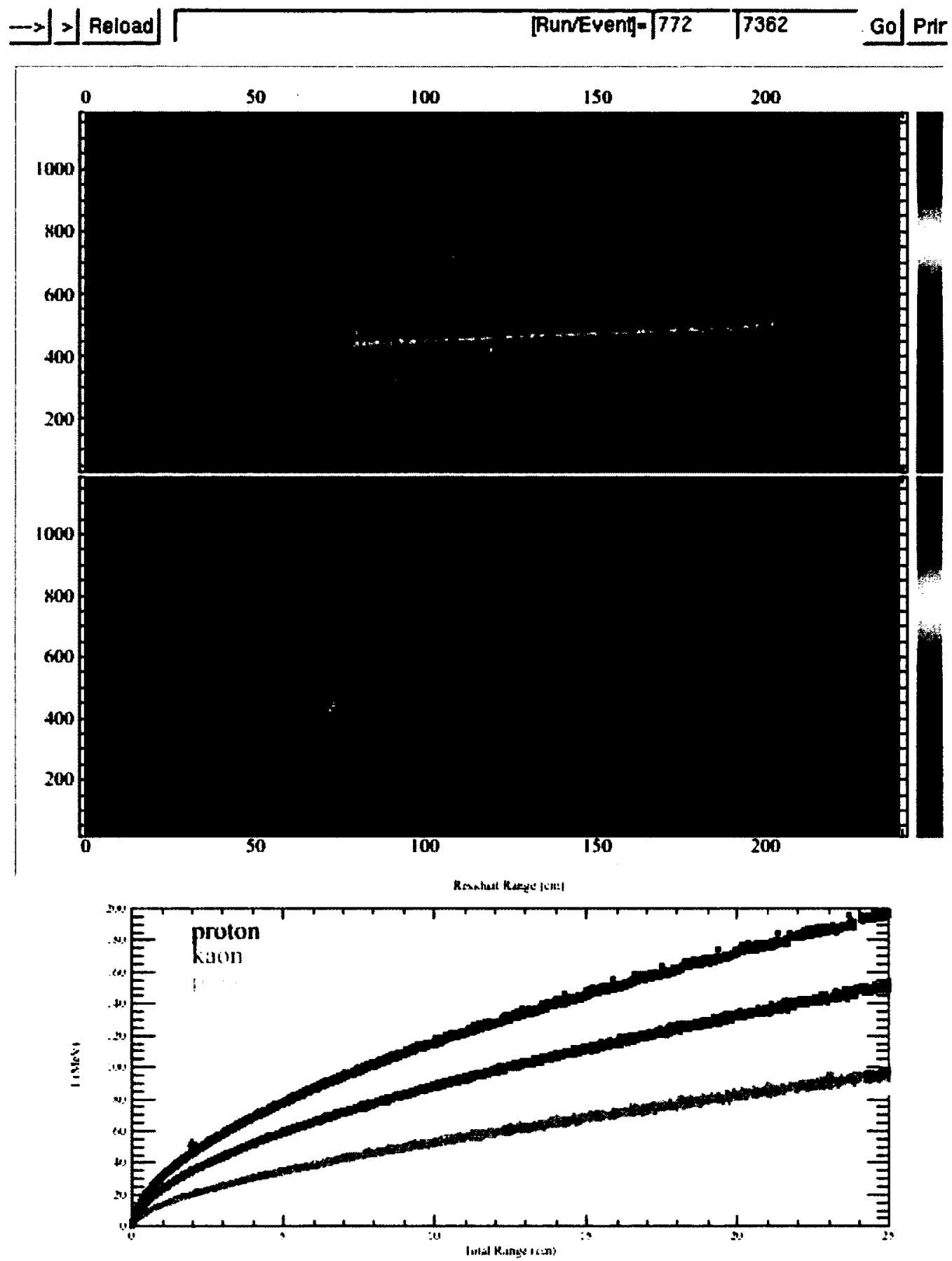

Figure 14.40: Event display showing a muon track with the reconstructed proton track at the vetex. 


\subsection{Efficiencies}

The percentage of $\mu+\mathrm{Np}$ events lost after each cut is reported in Table 14.5 for neutrinos and Table 14.6 anti-neutrinos. This percentage should be compared with the loss of all other events in the sample that are not $\mu+\mathrm{Np}$ events. It can be seen that each cut eliminates more of 'other' events than the sample we are interested in. In other words, the cuts try to eliminate as many events that are not $\mu+\mathrm{Np}$ as possible but at the same time to retain most of our sample.

\begin{tabular}{|l|c|c|}
\hline cuts & \% lost $\mu+\mathrm{Np}$ events & \% lost other events \\
\hline after FV & - & - \\
\hline after MM & 4.1 & 54.7 \\
\hline after VCluster & 22.2 & 64.8 \\
\hline after LineMerger & 12.8 & 33.5 \\
\hline after 3d Exiting Tracks & 2.9 & 32.2 \\
\hline
\end{tabular}

Table 14.5: Percentage of lost events after each sets of cuts for neutrinos in anti-neutrino mode.

\begin{tabular}{|l|c|c|}
\hline cuts & \% lost $\mu+\mathrm{Np}$ events & \% lost other events \\
\hline after FV & - & - \\
\hline after MM & 6.6 & 59.7 \\
\hline after VCluster & 12.5 & 50.8 \\
\hline after LineMerger & 6.2 & 27.3 \\
\hline after 3d Exiting Tracks & 2.4 & 13.1 \\
\hline
\end{tabular}

Table 14.6: Percentage of lost events after each sets of cuts for anti-neutrinos in anti-neutrino mode.

Efficiency of cuts for each multiplicity $N, \epsilon_{c u t s}^{N}$, was evaluated from MC according to Equation 14.6. Efficiency of scanning for each multiplicity $N, \epsilon_{s c a n}^{N}$, was evaluated from MC according to Equation 14.3. Efficiency of cuts is reported in Table 14.7 for neutrinos and Table 14.9 for anti-neutrinos. Efficiency of scanning is reported in Table 14.8 for neutrinos and Table 14.10 for anti-neutrinos. The second column in those tables shows the efficiencies for 'inclusive' sample, meaning that there is no requirement on the proton containment in the $\mu+\mathrm{Np}$ events. The third column of Tables 14.8 and Table 14.10 shows these efficiencies for events where all protons are contained. For the purpose of this analysis, only the efficiency numbers of the 'inclusive' sample are used, since we are not just interested in the contained events. However, the efficiencies for $\mu+\mathrm{Np}$ events with contained protons are shown since they really illustrate how well a scanner is doing in his/her selection. Recall 
that calorimetry requires all the protons to be contained and thus the scanner picks these events. From the reported numbers, It is seen that the scanner effectively selects events with contained protons. It is expected that the efficiency numbers for 'inclusive' $\mu+N p$ events are lower since the scanner rejects events that are not contained and thus the numerator of Equation 14.3 shrinks significantly after scanning. The only multiplicity not affected by containment is $\mathrm{N}=0$. The efficiency of scanning for $\mathrm{N}=1$ is higher for anti-neutrinos than for neutrinos. This is because energy spectrum is much higher for neutrinos than for anti-neutrinos. Thus, protons in $\mu+\mathrm{Np}$ neutrino events are more energetic and more likely to exit the TPC.

\begin{tabular}{|l|l|}
\hline Multiplicity & $\epsilon_{\text {cuts }}^{N}$ (inclusive) \\
\hline $0 \mathrm{p}+\mu$ & 0.83 \\
\hline $1 \mathrm{p}+\mu$ & 0.64 \\
\hline $2 \mathrm{p}+\mu$ & 0.39 \\
\hline $3 \mathrm{p}+\mu$ & 0.29 \\
\hline $4 \mathrm{p}+\mu$ & 0.19 \\
\hline
\end{tabular}

Table 14.7: Efficiency of cuts, $\epsilon_{c u t s}^{N}$ for $\mu+N p$ neutrino events for each multiplicity $N$. The term 'inclusive' refers to $\mu+\mathrm{Np}$ events without the restriction on proton containment.

\begin{tabular}{|l|l|l|}
\hline Multiplicity & $\epsilon_{\text {scan }}^{N}$ (inclusive) & $\epsilon_{\text {scan }}^{N}$ (contained) \\
\hline $0 \mathrm{p}+\mu$ & 0.99 & 0.99 \\
\hline $1 \mathrm{p}+\mu$ & 0.61 & 0.97 \\
\hline $2 \mathrm{p}+\mu$ & 0.67 & 0.97 \\
\hline $3 \mathrm{p}+\mu$ & 0.7 & 1 \\
\hline $4 \mathrm{p}+\mu$ & 0.7 & 1 \\
\hline
\end{tabular}

Table 14.8: Efficiency of scanning, $\epsilon_{s c a n}^{N}$ for $\mu+N p$ neutrino events for each multiplicity $N$. The second column shows efficiency of scanning for inclusive events (no restriction on proton containment) and the third shows it for sample with contained protons only. It is seen that the efficiency for contained protons is very high, while it is lower for inclusive sample. This behaviour is expected, as a scanner is supposed to pick out events with contained protons and thus the efficancy of this class of events is expected to be higher.

To determine the efficiency of calorimetric reconstruction four types of particles were generated with particle momentum uniformly distributed between 0 and $600 \mathrm{MeV} / \mathrm{c}$. An automated reconstruction was used to reconstruct these tracks. Events with at least one reconstructed track and reconstructed kinetic energy within $50 \%$ of the true kinetic energy were selected. This procedure was performed to ensure a track is well reconstructed. In the case of manual reconstruction performed on the data this is not a problem as each track 


\begin{tabular}{|l|l|}
\hline Multiplicity & $\epsilon_{\text {cuts }}^{N}$ (inclusive) \\
\hline $0 \mathrm{p}+\mu$ & 0.77 \\
\hline $1 \mathrm{p}+\mu$ & 0.63 \\
\hline $2 \mathrm{p}+\mu$ & 0.46 \\
\hline $3 \mathrm{p}+\mu$ & 0.35 \\
\hline $4 \mathrm{p}+\mu$ & 0.27 \\
\hline
\end{tabular}

Table 14.9: Efficiency of cuts, $\epsilon_{c u t s}^{N}$ for $\mu+N p$ anti-neutrino events for each multiplicity $N$. The term 'inclusive' refers to $\mu+\mathrm{Np}$ events without the restriction on proton containment.

\begin{tabular}{|l|l|l|}
\hline Multiplicity & $\epsilon_{\text {scan }}^{N}$ (inclusive) & $\epsilon_{\text {scan }}^{N}$ (contained) \\
\hline $0 \mathrm{p}+\mu$ & 0.96 & 0.96 \\
\hline $1 \mathrm{p}+\mu$ & 0.86 & 0.96 \\
\hline $2 \mathrm{p}+\mu$ & 0.82 & 0.86 \\
\hline $3 \mathrm{p}+\mu$ & 0.67 & 0.87 \\
\hline $4 \mathrm{p}+\mu$ & 0.67 & 0.87 \\
\hline
\end{tabular}

Table 14.10: Efficiency of scanning, $\epsilon_{s c a n}^{N}$, for $\mu+\mathrm{Np}$ anti-neutrino events for each multiplicity $\mathrm{N}$. It is seen that the efficiency for contained protons is very high, while it is lower for inclusive sample. This behaviour is expected, as a scanner is supposed to pick out events with contained protons and thus the efficancy of this class of events is expected to be higher.

is reconstructed in a semi-automated way as described in Section 14.2.5. The confusion matrix was generated with events having a well reconstructed track with the automated reconstruction as shown in Figure 14.41. It clearly shows the proton identification efficiency is very high, being $97 \%$.

\begin{tabular}{|c|c|c|c|c|}
\hline & & enerate & & \\
\hline & Proton & Kaon & Pion & Muon \\
\hline Proton & 0.97 & 0.15 & 0.05 & 0 \\
\hline Kaon & 0.03 & 0.60 & 0.09 & 0.01 \\
\hline Pion & 0 & 0.06 & 0.25 & 0.28 \\
\hline Muon & 0 & 0.20 & 0.61 & 0.71 \\
\hline
\end{tabular}

Figure 14.41: Confusion matrix for protons, kaon, muons and pions generated by reconstructing simulated particles. It is seen that the efficiency of reconstructing protons is $97 \%, 3 \%$ of the time proton is confused with a kaon. 


\subsection{Background}

The background, such as Neutral Current (NC), Wrong Sign (WS), and $\pi^{0}$ with both gammas that do not convert in the ArgoNeuT, should also be subtracted from each multiplicity bin after calorimetry performance. Note, that as discussed above there is no need to perform this subtraction in the MC-based method as the true fractions of events already take the background into account. The percentage of these different backgrounds is calculated from the scanned MC sample and shown in Table 14.11 for neutrinos and Table 14.12 for anti-neutrinos. These percentages are applied to number of events 'after scanning' since they were also calculated on the MC sample 'after scanning' and then subtracted from the sample 'after calorimetry'.

\begin{tabular}{|l|c|c|c|}
\hline Multiplicity & \% of NC background & \% of WS background & \% of pi0 background \\
\hline $0 \mathrm{p}+\mu$ & 7 & 7.9 & 3.5 \\
\hline $1 \mathrm{p}+\mu$ & 1 & 3.5 & 5 \\
\hline $2 \mathrm{p}+\mu$ & 2.2 & 0 & 11.1 \\
\hline $3 \mathrm{p}+\mu$ & 0 & 0 & 9 \\
\hline $4 \mathrm{p}+\mu$ & 0 & 0 & 0 \\
\hline
\end{tabular}

Table 14.11: Number of background events for $\mu+\mathrm{Np}$ sample of neutrinos in anti-neutrino mode.

\begin{tabular}{|l|c|c|c|}
\hline Multiplicity & \% of NC background & \% of WS background & \% of pi0 background \\
\hline $0 \mathrm{p}+\mu$ & 0.73 & 0.24 & 3.65 \\
\hline $1 \mathrm{p}+\mu$ & 0.6 & 0.6 & 7 \\
\hline $2 \mathrm{p}+\mu$ & 0 & 0 & 2.8 \\
\hline $3 \mathrm{p}+\mu$ & 0 & 0 & 0 \\
\hline $4 \mathrm{p}+\mu$ & 0 & 0 & 0 \\
\hline
\end{tabular}

Table 14.12: Number of background events for $\mu+\mathrm{Np}$ sample of anti-neutrinos in anti-neutrino mode.

The two methods should give the same results assuming that MC models the data correctly. However, it is seen that pi+ and pi- background calculation based on MC differs significantly from that obtained from calorimetry. The background from calorimetry is estimated by counting the number of events reconstructed with pions in each bin and dividing by the total number of events in that bin after scanning. As an example, $\mathrm{MC}$ predicts $17 \%$ pion background for $1 \mathrm{p}$ bin for anti-neutrinos but calorimetry calculation gives about $10 \%$. For $2 \mathrm{p}, \mathrm{MC}$ predicts $28.8 \%$ background and calorimetry only $11 \%$. One question that should immediately be addressed is whether the higher pion background in 
MC is not attributed to very low energy pions that could not be detected in the data. A plot of pion momentum distributions for events that passed automated cuts is shown in Figure 14.42. Events with the lowest pion momentum were examined again, examples of them are shown in the Figure 14.43. All of them have visible tracks consisting of at least 2 hits in both planes and thus can be reconstructed by using the calorimetry method.

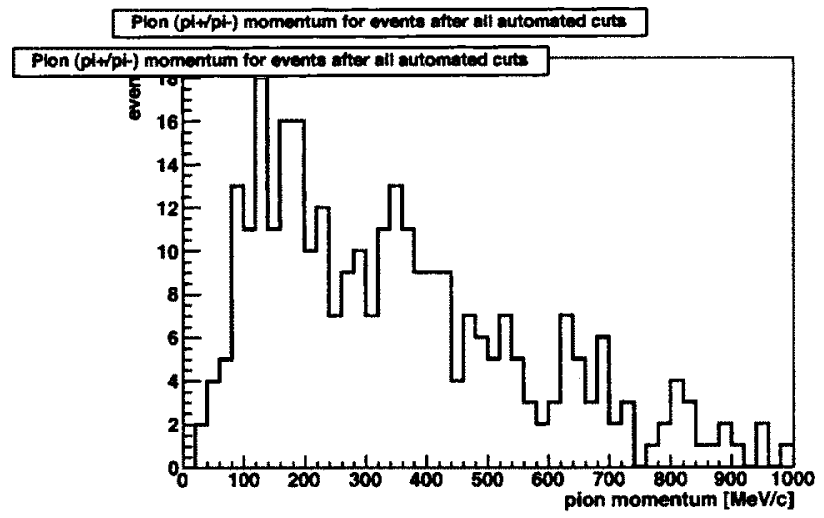

Figure 14.42: Distribution of pion momentum for MC events that passed automated cuts ( antineutrinos in anti-neutrino mode) 


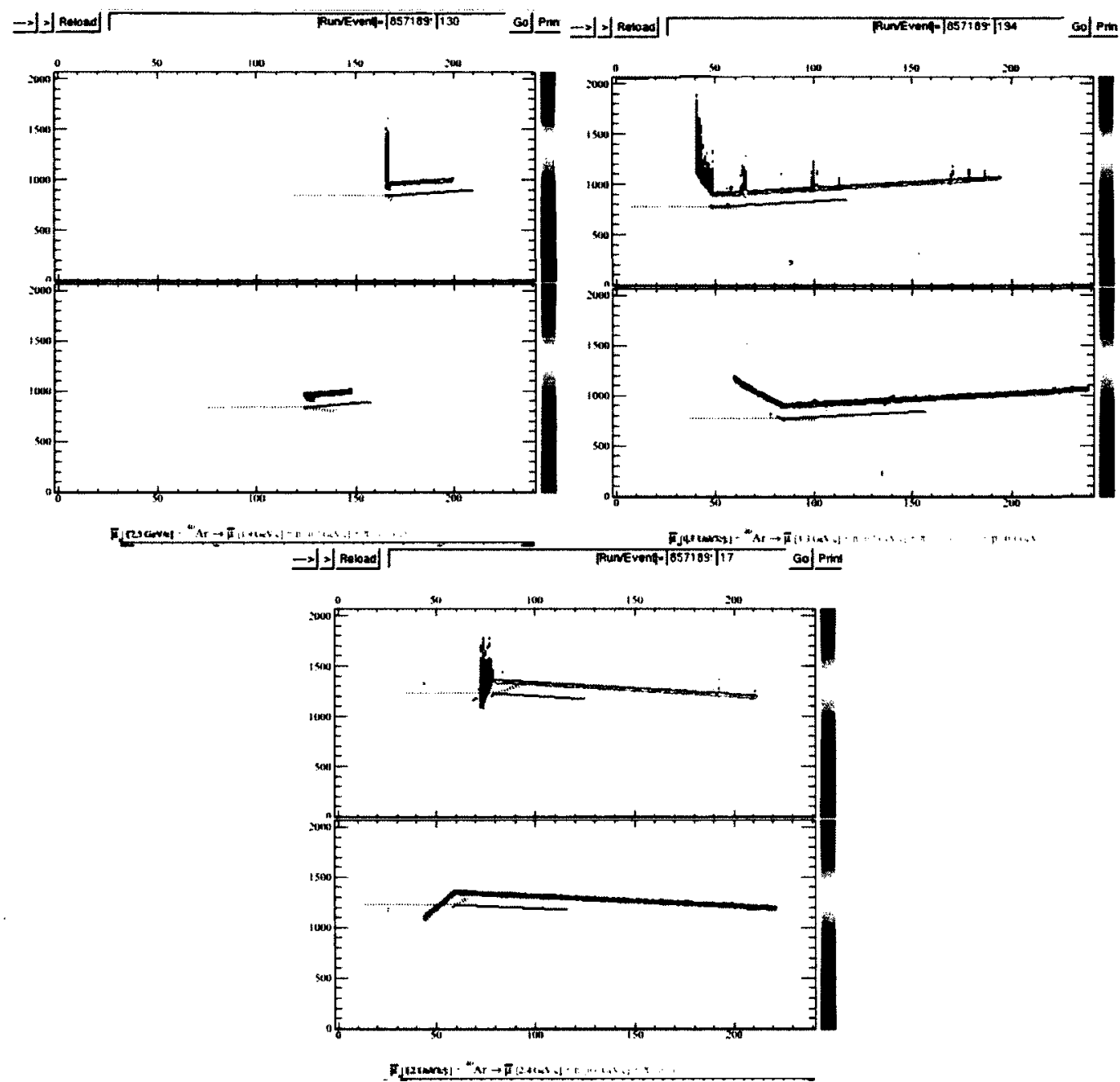

Figure 14.43: Examples of events with very low energy pions, on the order of $0.1 \mathrm{GeV} / \mathrm{c}$

Thus, it can be concluded that MC does not model the data correctly and the databased method should be used for this analysis. In other words, calorimetry will be used for pion background estimation. Other background (NC,WS, $\pi^{0}$ that did not convert) will be subtracted based on MC as discussed above. Wrong sign background is related to the MINOS efficiency of correctly recognizing the sign of the muon. It is higher for neutrino as MINOS reconstruction was tuned for $\mu^{+}$. Furthermore, neutrinos in anti-neutrino mode have higher energy and thus their curvature in the magnetized MND is smaller making the sign selection more challenging. The NC and $\pi^{0}$ are model dependent. 


\subsection{Flux}

The main elements of modeling the NuMI neutrino beam line is a simulation of the hadrons produced by $120 \mathrm{GeV} / \mathrm{c}$ protons and their propagation through the focusing horns, the decay pipe and through the primary beam absorber. FLUKA Monte Carlo is employed for the calculation of the production of secondary mesons in the NuMI target and the simulation of the beam line [119]. A detailed description of the simulation can be found in [120].

The flux employed in this analysis is shown in Figure 14.44 for neutrinos and Figure 14.45 for anti-neutrinos in the anti-neutrino mode. The systematic error on the flux stems from the uncertainties in hadron production in the NuMI target and beamline, and from modeling of the beam line focusing and geometry. The hadron production dominates the error, below the $10 \mathrm{GeV}$ of neutrino energy it is on the order of $10 \%-15 \%$ and is set conservatively at $15 \%$ in this range, above this energy range it is taken to be $30 \%[121,122]$. A dedicated group to study this error further has been set up, however, no better estimate is available at this time.

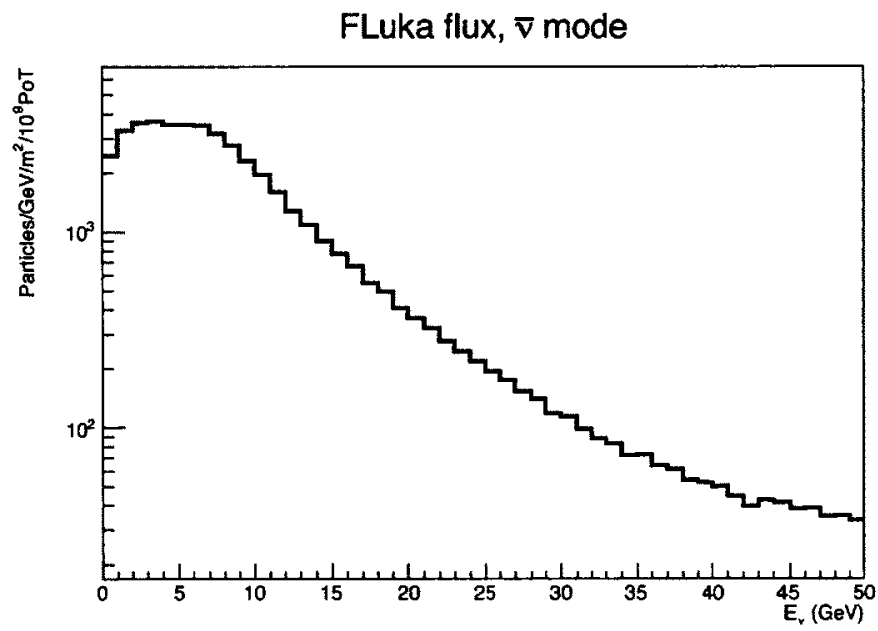

Figure 14.44: The flux for neutrinos in anti-neutrino mode.

\subsection{Protons on Target}

The number of POT is required to scale the $\mathrm{MC}$ and compare absolute rates of events with the data. The Fermilab accelerator division provides the number of protons at the beamline 


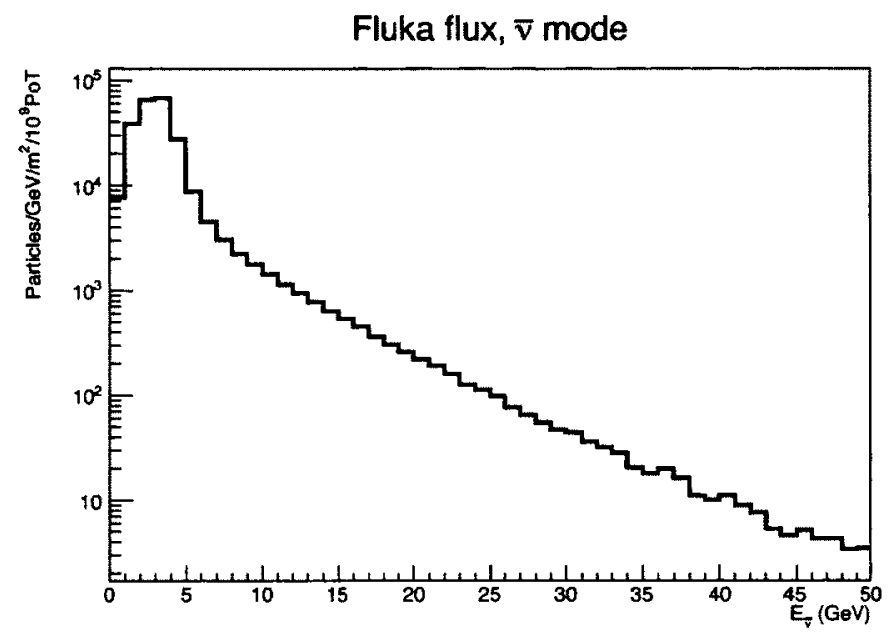

Figure 14.45: The flux for anti-neutrinos in anti-neutrino mode.

closest to the target. ArgoNeuT collected a total of $1.24 \times 10^{20}$ POT in anti-neutrino mode. Since this analysis requires matching of muons with MINOS Near Detector, the number of POT when both detectors were active should be considered. There was a total of $1.2 \times 10^{20}$ POT used in this analysis.

\subsection{Results}

All the presented results will be based on the data-based method as the pion background was found not to be modeled correctly based on the MC as discussed in Section 14.4.

\subsubsection{Proton multiplicity}

Data and $\mathrm{MC}$ comparison in terms of proton multiplicity for $\mu+\mathrm{Np}$ events and the percentage of total number of events are shown in the Tables 14.13 for neutrinos and 14.14 for antineutrinos in anti-neutrino mode. Comparison is done up to proton multiplicity of 4 , since it was the highest multiplicity event found in the data. Higher multiplicities for the $\mathrm{MC}$ are included in the total sum. The error on the $\mathrm{MC}$ is dominated by the flux uncertainty as addressed in Section 14.5. The errors on the data are explained in the subsection 'Error Analysis' below. Considering only the mean values, it can be seen that there is a bigger percentage of $0 p, 1 p$ and $2 p$ events in the data for neutrinos than predicted by GENIE. The data points fall within the MC error for proton multiplicities $N=0,1,2$. For 
$\mathrm{N}=3,4$ GENIE predicts more events. For anti-neutrinos, there is also a bigger percentage of $0 \mathrm{p}$ and $1 \mathrm{p}$ events in the data as compared to the MC. The MC nicely models the rate of $1 p$ events but predicts more events for other multiplicities. Overall it can be seen that for both neutrinos and anti-neutrinos there is less data than predicted by GENIE by $27 \%$ for anti-neutrinos in anti-neutrino mode and $25 \%$ for neutrinos in anti-neutrino mode by considering the mean values of the distributions.

The absolute rates and the break down of MC events in terms of CCQE, CCRES and DIS events are shown in Figure 14.46 for neutrinos and in Figure 14.47 for anti-neutrinos in anti-nu mode. It is seen that there is a substantial contribution from non-CCQE events as the multiplicity increases. The percentage of non-CCQE events as a function of multiplicity $\mathrm{N}$ is shown in Table 14.15 for neutrinos and anti-neutrinos. These high fractions of nonCCQE events further emphasize the need for topological analysis and not correcting for interactions modeled by a MC. In Cherenkov detectors all of the $\mu+\mathrm{Np}$ events would be classified as "CCQE-like" events.

The only standard interactions in the $\mu+\mathrm{Np}$ class of topological events are $\mu+1 \mathrm{p}$ for neutrinos and $\mu+0 \mathrm{p}$ for anti-neutrinos. The fact that higher multiplicity of protons at the vertex is observed or no proton at the vertex in the case of neutrinos is an indication of final state interactions and/or multi-nucleon correlation. These were previously ignored by other detectors as they cannot be detected by them. The rates of proton multiplicity along with fractions of events as a function multiplicity can be used to tune MC generators and discriminate among different models. Furthermore, ratios of different multiplicities can be used for the indication of the size of the nuclear effects. For example, the ratio of $(1 p / 0 p)_{D A T A}=0.35$ while $(1 p / 0 p)_{M C}=0.29$ for anti-neutrinos, and $(0 p / 1 p)_{D A T A}=0.40$ while $(0 p / 1 p)_{M C}=0.35$ for neutrinos.

\begin{tabular}{|c|c|l|c|l|}
\hline Multiplicity & Genie Expectation & $\begin{array}{l}\text { Genie } \% \\
\text { of Total }\end{array}$ & DATA & $\begin{array}{l}\text { DATA \% } \\
\text { of Total }\end{array}$ \\
\hline $0 \mathrm{p}+\mu$ & $51.4 \pm 1.8 \pm 5$ & $15 \%$ & $49.9 \pm 8.4 \pm 0.5$ & $20 \%$ \\
\hline $1 \mathrm{p}+\mu$ & $145.8 \pm 3 \pm 14.2$ & $43.5 \%$ & $126.6 \pm 19 \pm 1.5$ & $50 \%$ \\
\hline $2 \mathrm{p}+\mu$ & $44.5 \pm 1.7 \pm 4.3$ & $13 \%$ & $53.8 \pm 15.4 \pm 0.3$ & $21 \%$ \\
\hline $3 \mathrm{p}+\mu$ & $27.6 \pm 1.4 \pm 2.7$ & $8 \%$ & $14.7 \pm 9.1 \pm 0.0$ & $6 \%$ \\
\hline $4 \mathrm{p}+\mu$ & $18.4 \pm 1 \pm 1.8$ & $5.5 \%$ & $7.5 \pm 7.7 \pm 0.0$ & $3 \%$ \\
\hline Total (including $>4 \mathrm{p}$ ) & $335.5 \pm 4.7 \pm 26.1$ & $-\%$ & $252.5 \pm 27.2 \pm 1.6$ & $-\%$ \\
\hline
\end{tabular}

Table 14.13: Data comparison with GENIE in terms of proton multiplicity of $\mu+N$ p events and fractions of events for neutrinos in anti-neutrino mode. The first number following the " \pm " represents the statistical error and the second one the systematic error. 


\begin{tabular}{|c|c|l|c|l|}
\hline Multiplicity & Genie Expectation & $\begin{array}{l}\text { Genie } \% \\
\text { of Total }\end{array}$ & DATA & $\begin{array}{l}\text { DATA \% } \\
\text { of Total }\end{array}$ \\
\hline $0 \mathrm{p}+\mu$ & $510 \pm 5.8 \pm 40$ & $58.4 \%$ & $431 \pm 27.2 \pm 10.6$ & $67.7 \%$ \\
\hline $1 \mathrm{p}+\mu$ & $149.4 \pm 3 \pm 11.8$ & $17 \%$ & $150.8 \pm 18.9 \pm 2.1$ & $23.7 \%$ \\
\hline $2 \mathrm{p}+\mu$ & $69 \pm 2 \pm 5.5$ & $8 \%$ & $41.3 \pm 11.4 \pm 0.3$ & $6.4 \%$ \\
\hline $3 \mathrm{p}+\mu$ & $48.5 \pm 1.8 \pm 3.9$ & $5.5 \%$ & $8.6 \pm 6.2 \pm 0.0$ & $1.4 \%$ \\
\hline $4 \mathrm{p}+\mu$ & $37 \pm 1.5 \pm 3$ & $4.2 \%$ & $5.6 \pm 5.6 \pm 0.1$ & $1 \%$ \\
\hline Total (including $>4 \mathrm{p})$ & $872.4 \pm 7.6 \pm 67$ & $-\%$ & $637.3 \pm 36 \pm 10.8$ & $-\%$ \\
\hline
\end{tabular}

Table 14.14: Data comparison with GENIE in terms of proton multiplicity of $\mu+N p$ events and fractions of events for anti-neutrinos in anti-neutrino mode. The first number following the " \pm " represents the statistical error and the second one the systematic error.

\begin{tabular}{|c|c|c|}
\hline Multiplicity & \% of non-CCQE GENIE $\nu$ events & \% of non-CCQE GENIE $\bar{\nu}$ events \\
\hline $0 \mathrm{p}+\mu$ & 2.45 & 10.36 \\
\hline $1 \mathrm{p}+\mu$ & 6.46 & 39.74 \\
\hline $2 \mathrm{p}+\mu$ & 60.15 & 73.97 \\
\hline $3 \mathrm{p}+\mu$ & 81.01 & 83.17 \\
\hline $4 \mathrm{p}+\mu$ & 83.03 & 89.59 \\
\hline $5 \mathrm{p}+\mu$ & 82.35 & 91.86 \\
\hline $6 \mathrm{p}+\mu$ & 86.06 & 96.35 \\
\hline $7 \mathrm{p}+\mu$ & 96.52 & 95.17 \\
\hline $8 \mathrm{p}+\mu$ & 96.51 & 99.02 \\
\hline $9 \mathrm{p}+\mu$ & 100 & 100 \\
\hline $10 \mathrm{p}+\mu$ & 96.88 & 100 \\
\hline
\end{tabular}

Table 14.15: Fractions of non-CCQE events as a function of proton multiplicity in $\mu+\mathrm{Np}$ events for neutrinos and anti-neutrinos in anti-neutrino mode. 
Neutrinos In Anti-neutrino mode

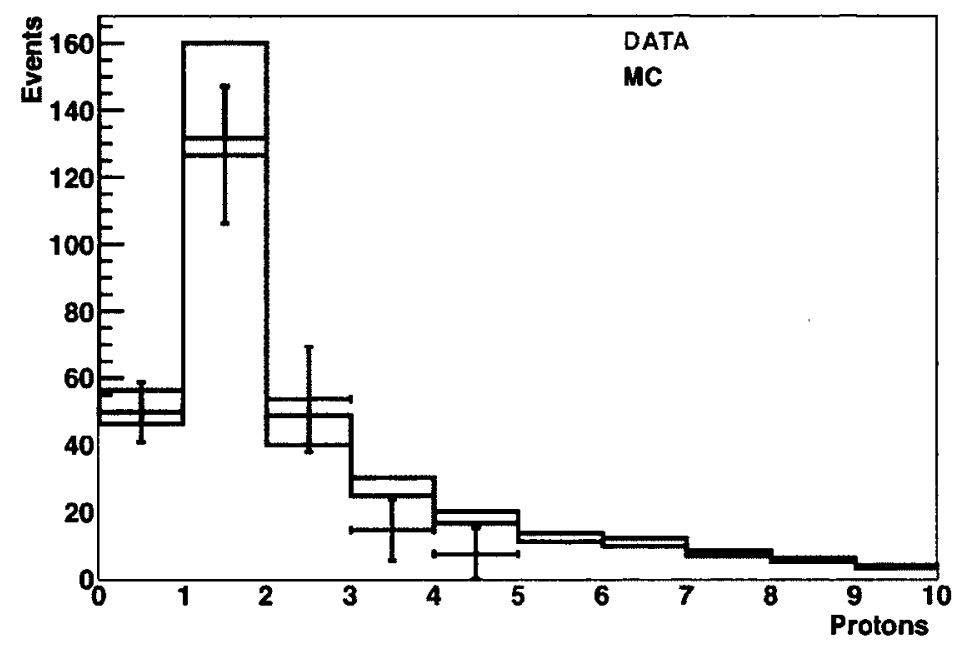

Neutrinos in Anti-neutrino mode

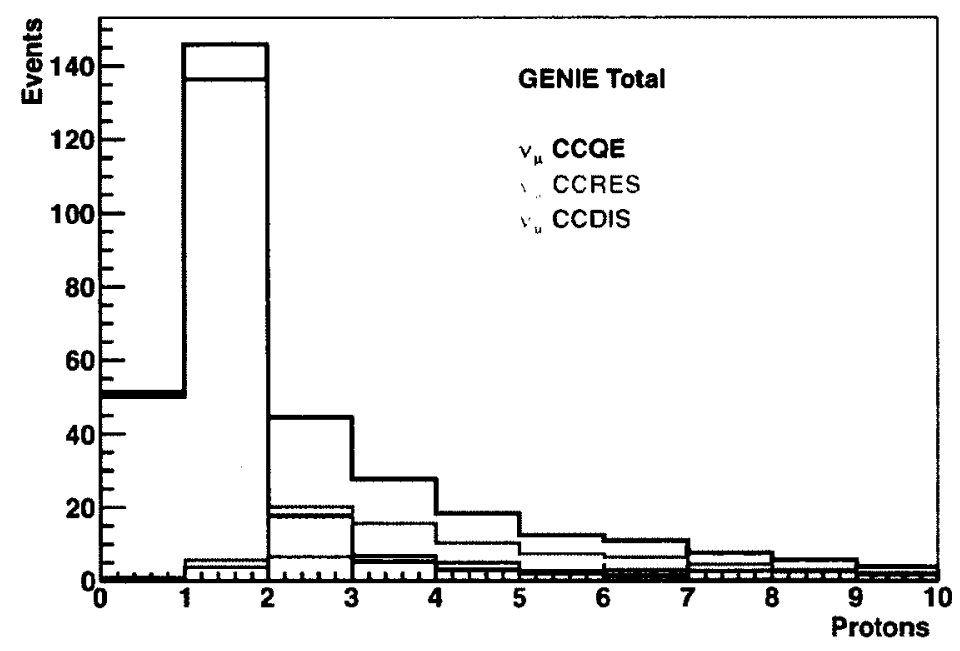

Figure 14.46: Distribution of proton multiplicities for neutrinos in the data and comparison with GENIE expectation (top). The error on the data includes both the statistical and systematic uncertainty. The MC band (in grey) represents the large uncertainty coming from the flux. The bottom plot shows the distribution of proton multiplicities as modeled by the MC with various contributions, no uncertainty is shown for the clarity of the plot. 


\section{Anti-neutrinos in Anti-neutrino mode}

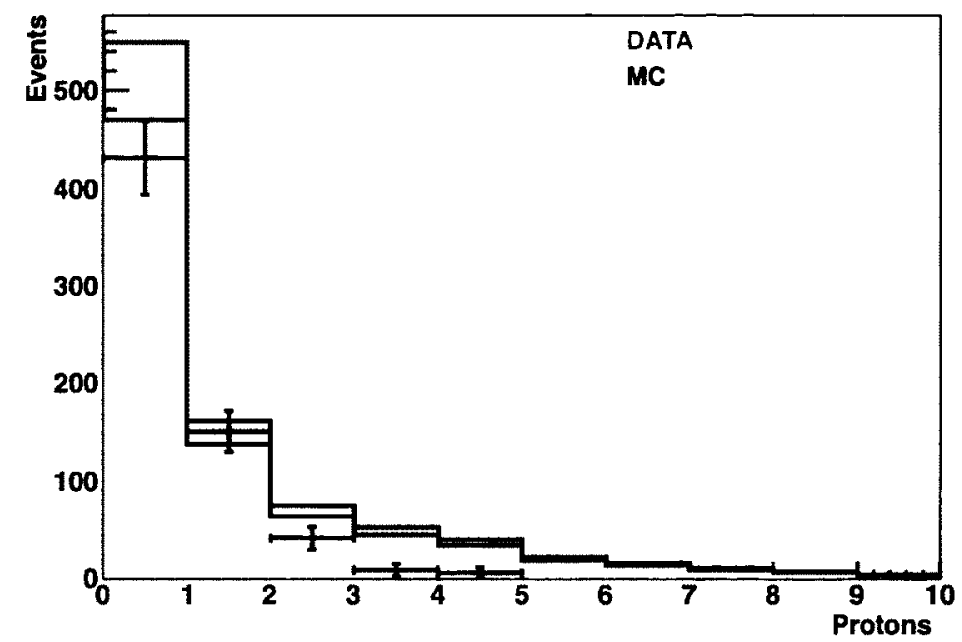

Anti-neutrinos in Anti-neutrino mode

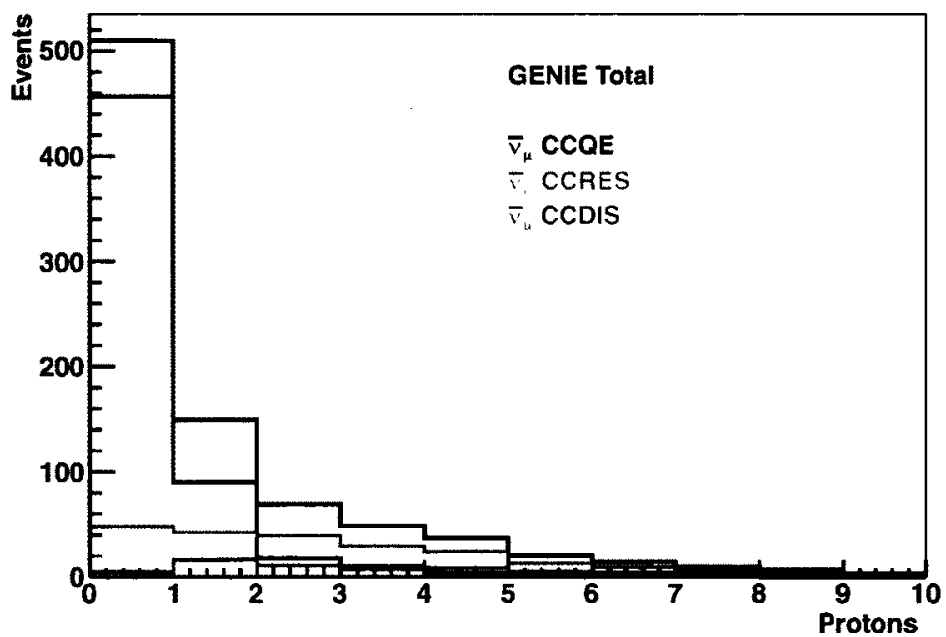

Figure 14.47: Distribution of proton multiplicities for anti-neutrinos in the data and comparison with GENIE expectation (top). The error on the data includes both the statistical and systematic uncertainty. The MC band (in grey) represents the large uncertainty coming from the flux. The bottom plot shows the distribution of proton multiplicities as modeled by the MC with various contributions, no uncertainty is shown for the clarity of the plot. 


\section{Error Analysis}

The analysis is statistics-limited but several most significant sources of systematic errors have been taken into consideration. The number of events after automated cuts, scanning and calorimetry for neutrinos is 126 and for anti-neutrinos 443 .

The total statistical error that takes into account the number of events after calorimetry, error from efficiency of cuts and efficiency of scanning after appropriate error propagation is shown in Table 14.16. It is worth noting that it is largely dominated by the number of events obtained after performing calorimetry, which are shown in Table 14.3 for neutrinos and in Table 14.4 for anti-neutrinos. It is seen that error increases with proton multiplicity. This is expected and attributed to the decreasing number of events for increasing number of proton multiplicity.

\begin{tabular}{|l|c|c|}
\hline multiplicity N & Stat. frac. error for neutrinos & Stat. frac. error for anti-neutrinos \\
\hline 0 & 0.169 & 0.063 \\
\hline 1 & 0.149 & 0.125 \\
\hline 2 & 0.286 & 0.275 \\
\hline 3 & 0.614 & 0.722 \\
\hline 4 & 1.023 & 1.011 \\
\hline
\end{tabular}

Table 14.16: Total statistical fractional errors for neutrinos and anti-neutrinos.

The error associated with the fiducial volume cut is taken from CC-inclusive analysis as it is the same as used in this analysis. It was found to be $1 \%$ after calculating the reconstructed Monte Carlo simulation rate by increasing and then decreasing fiducial volume by $1 \mathrm{~cm}$ on all sides [117]. Besides the background being sensitive to the fiducial volume cut, it is also affected by visual scanning. A sample of about 300 events was scanned by two scanners independently and their results in terms of scanner's efficiency for each multiplicity $\mathrm{N}$ compared. The biggest difference between their efficiency is conservatively taken as the systematic error and is found to be $5.1 \%$. The systematic error associated with the calorimetric reconstruction is determined to be $3 \%$ as proton is confused with a kaon $3 \%$ of the time, more details of this procedure are described in Section 14.4. The systematic error associated with POT counting is at $1 \%$ [118].

The total fractional error for each multiplicity $\mathrm{N}$ obtained after adding all the errors in quadrature is reported in Table 14.17 for neutrinos and anti-neutrinos. 


\begin{tabular}{|l|c|c|}
\hline multiplicity $\mathrm{N}$ & Tot. frac. error for neutrinos & Tot. frac. error for anti-neutrinos \\
\hline 0 & 0.179 & 0.088 \\
\hline 1 & 0.162 & 0.139 \\
\hline 2 & 0.292 & 0.282 \\
\hline 3 & 0.617 & 0.724 \\
\hline 4 & 1.025 & 1.013 \\
\hline
\end{tabular}

Table 14.17: Total fractional errors for neutrinos and anti-neutrinos obtained by adding systematic error with all systematic in quadrature.

\subsubsection{Muon Kinematics}

Understanding of muon kinematics is a very important aspect of neutrino physics as neutrino energy calculations rely heavily on muon reconstruction. Figure 14.48 shows muon angle (left) and muon momentum (right), for all neutrino events after calorimetric reconstruction, and comparison with GENIE. The plots are area normalized with the background subtracted, errors are statistical only. The discussion of background is given in Section 14.4 and more discussion on the specific technique used for the plots presented here can be found in the sub-section 'Background' below. It can be seen that the MC predicts a much lower muon angle with the mean of 5.8 degrees, data, on the other hand, shows a much higher mean of 9.8 degrees. The momentum of the muon is lower in the data than predicted by the MC, which is expected following the angular distribution plot, as higher angle muons correspond to less energetic tracks. This behaviour is also seen in $\mu+0 p$ and $\mu+1 p$ neutrino events as shown in Figures 14.49 and 14.50 respectively. Higher multiplicity events are not shown due to low statistics. A quite different pattern is observed in anti-neutrino $\mu+N p$ events as shown in Figure 14.51 where GENIE models the data very well. A good agreement is seen for both the mean of the angular and momentum distributions as well as the shape. The same distributions for $\mathrm{N}=0$ and $\mathrm{N}=1$ multiplicities are shown in Figures 14.52 and 14.53 for anti-neutrinos. Here, again the distributions are modeled quite well with a bit better shape representation for $\mathrm{N}=0$ than for $\mathrm{N}=1$ events, which are at slightly higher energies. A possible explanation for data/MC discrepancy seen in neutrinos is correlation between nucleons, which are not modeled at all in GENIE. In the multi-nucleon processes a substantial amount of energy is transferred to the hadrons and as a result less to the outgoing lepton. This process would be more significant for higher energy neutrinos as more energy is required for these interactions, which agrees with what is observed. 

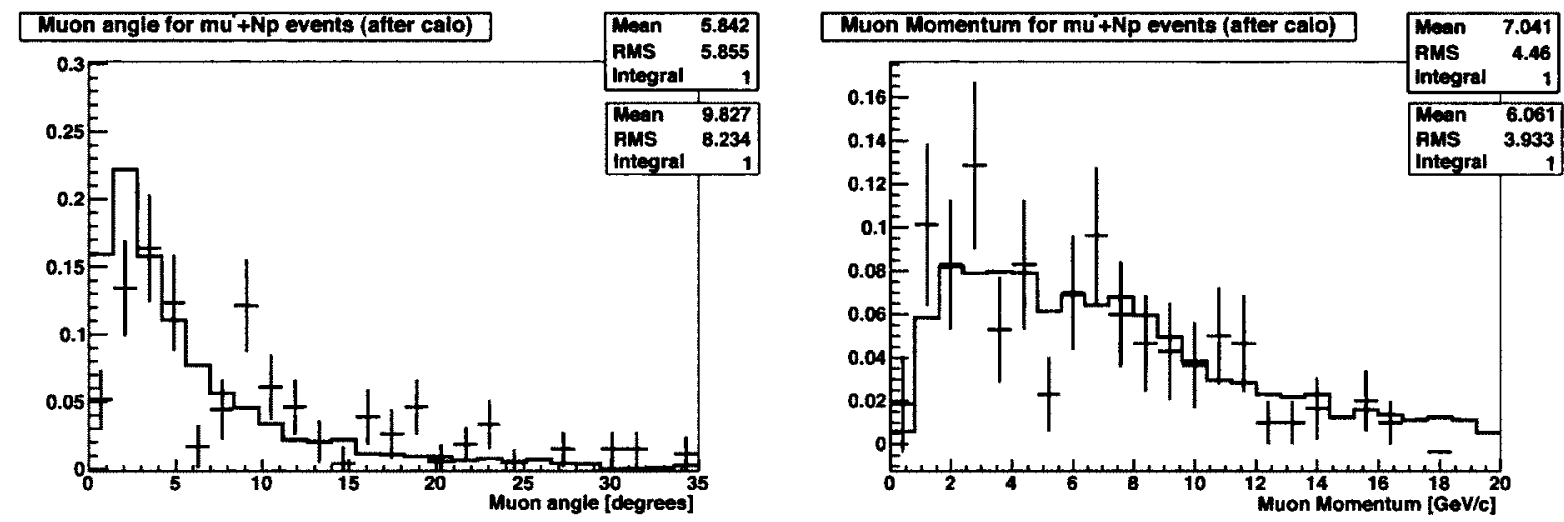

Figure 14.48: Muon angle and momentum distribution for $\mu+\mathrm{Np}$ neutrino events. Data events entering in these plots come after calorimetry reconstruction (background subtracted) and the MC corresponds to contained $\mu+\mathrm{Np}$ after all cuts.
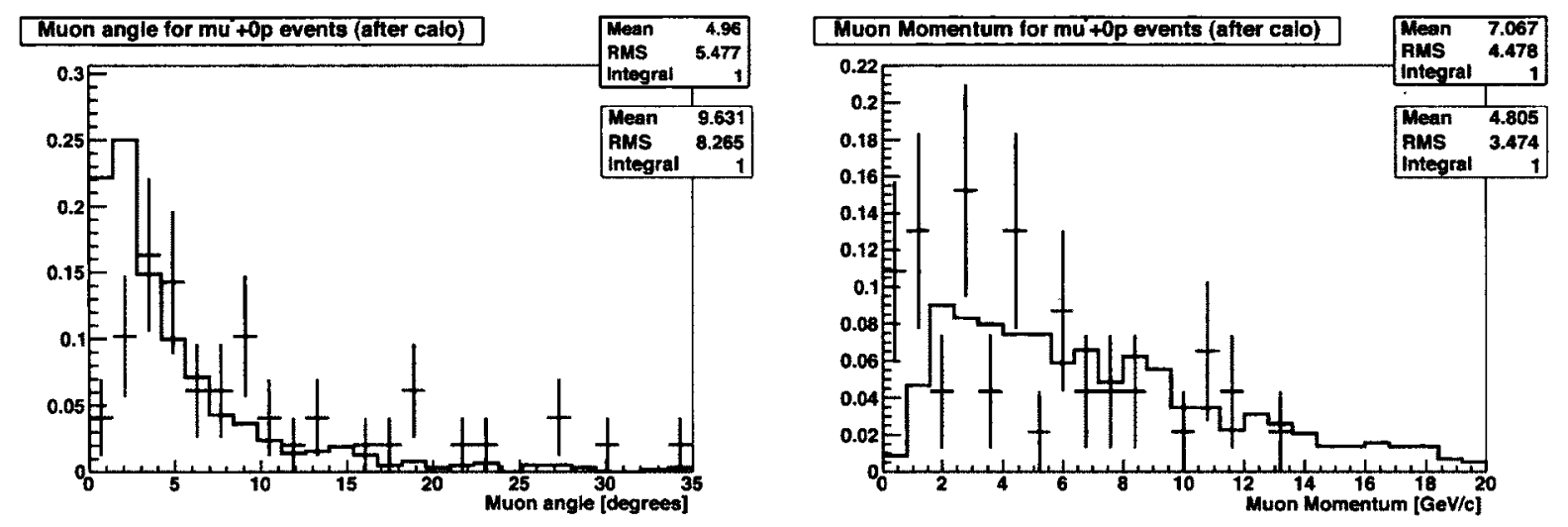

Figure 14.49: Muon angle and momentum distribution for $\mu+0 \mathrm{p}$ neutrino events. Data events entering in these plots come after calorimetry reconstruction and the $\mathrm{MC}$ corresponds to contained $\mu+0 \mathrm{p}$ after all cuts. 

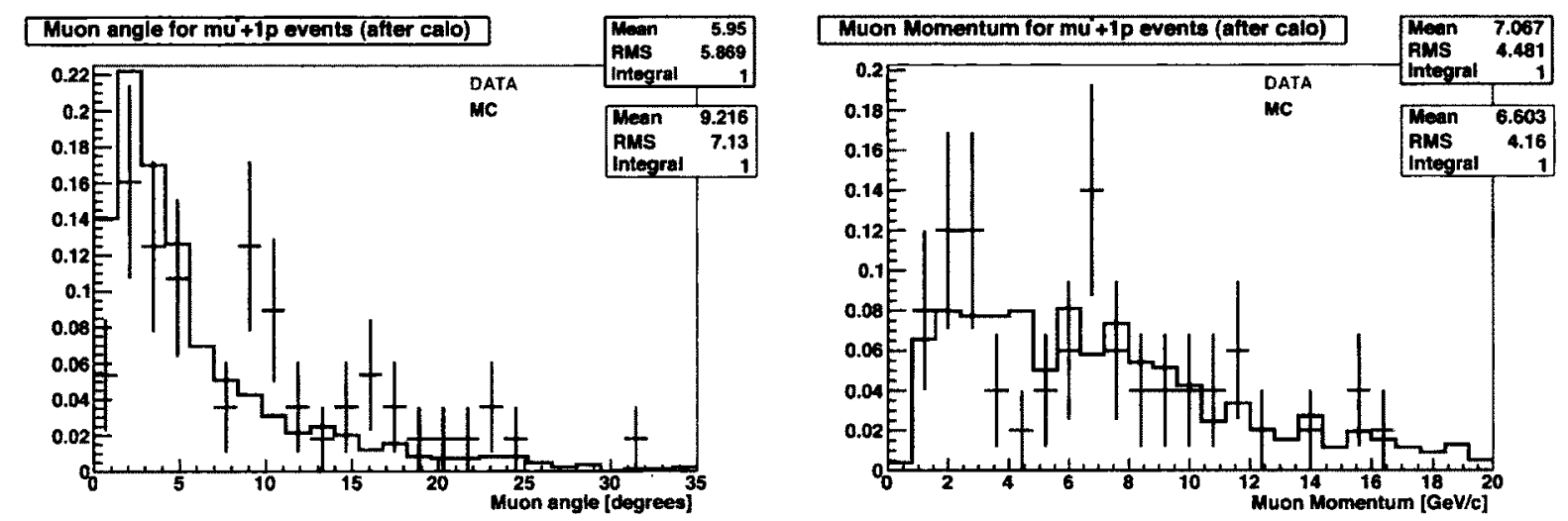

Figure 14.50: Muon angle and momentum distribution for $\mu+1 \mathrm{p}$ neutrino events. Data events entering in these plots come after calorimetry reconstruction and the $\mathrm{MC}$ corresponds to contained $\mu+1$ p after all cuts.
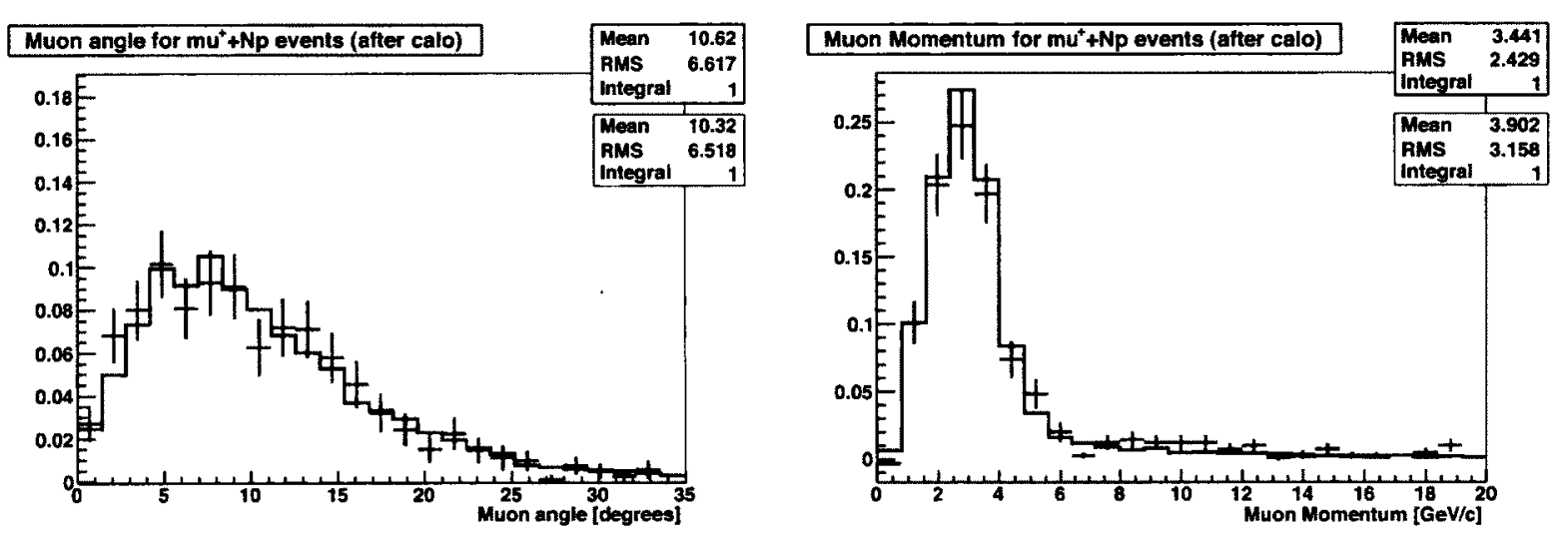

Figure 14.51: Muon angle and momentum distribution for $\mu+\mathrm{Np}$ anti-neutrino events. Data events entering in these plots come after calorimetry reconstruction (background subtracted), the $\mathrm{MC}$ corresponds to contained $\mu+\mathrm{Np}$ after all cuts. 

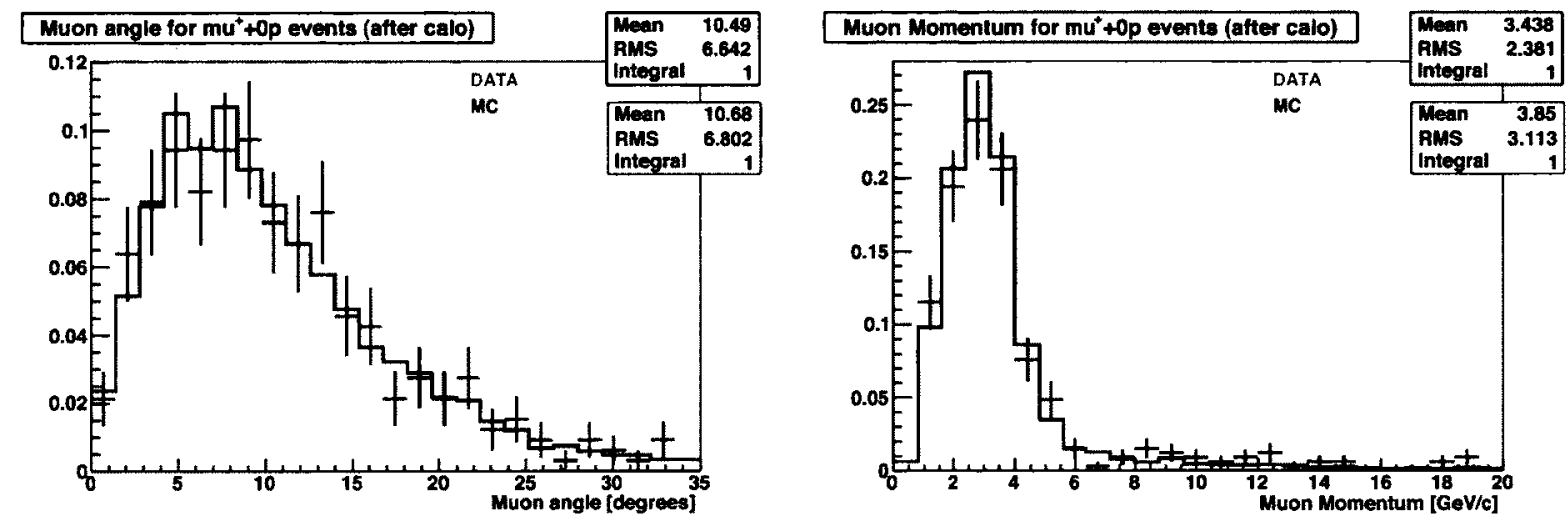

Figure 14.52: Muon angle and momentum distribution for $\mu+0 \mathrm{p}$ anti-neutrino events. Data events entering in these plots come after calorimetry reconstruction, the $\mathrm{MC}$ corresponds to contained $\mu+0 \mathrm{p}$ after all cuts.
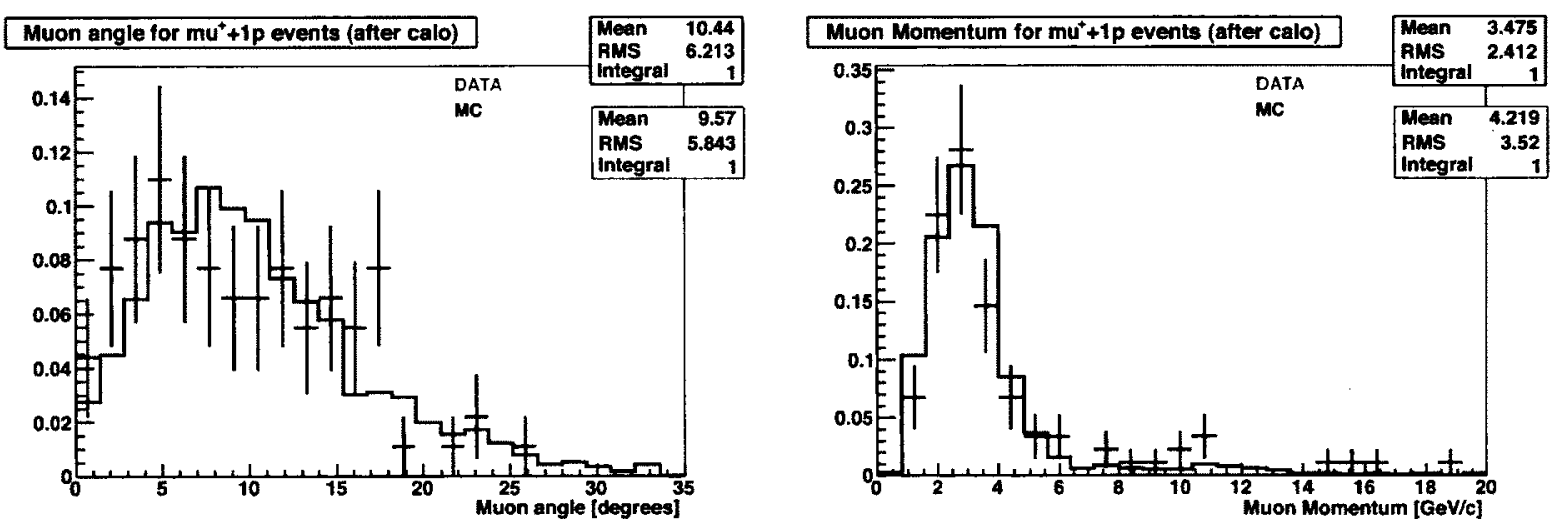

Figure 14.53: Muon angle and momentum distribution for $\mu+1 \mathrm{p}$ anti-neutrino events. Data events entering in these plots come after calorimetry reconstruction, the $\mathrm{MC}$ corresponds to contained $\mu+1 p$ after all cuts. 


\section{Background}

The background was evaluated based on the visual scanning method, described in Section 14.2.3, on the 2000-event-MC-sample. The MC-truth was employed to determine the background in the sample that the scanner marked as candidate $\mu+N p$ events. The fraction of different background for each multiplicity is given in Section 14.4. The total background for neutrino $\mu+N p$ events was calculated to be $12.8 \%$ and $5.4 \%$ for anti-neutrinos. The data after calorimetric reconstruction and the corresponding background for muon momentum distribution in neutrino $\mu+N p$ events is shown in Figure 14.54. The corresponding angular distribution is given in Figure 14.55. The data after calorimetric reconstruction and the corresponding background for muon momentum distribution in anti-neutrino $\mu+N p$ events is shown in Figure 14.56. The corresponding angular distribution is given in Figure 14.57.

The background is quite small and due to the very small statistics, both of the number of events in the data after calorimetric reconstruction and the number of background events derived from visual scanning of the $\mathrm{MC}$, it has only been subtracted from the plots including all proton multiplicities. The distributions after the indicated backgrounds, Figures 14.5414.57, subtractions are shown in Figure 14.48 and Figure 14.51, respectively. It is seen that the background has little significance on the mean value of the distributions.

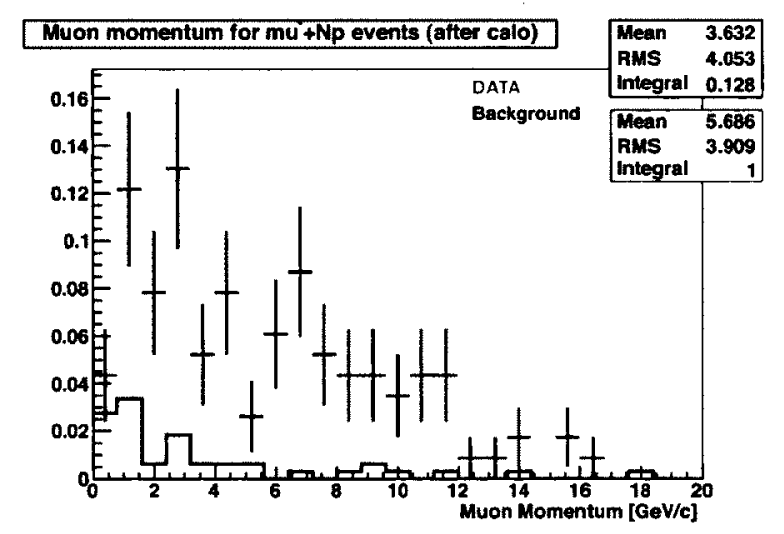

Figure 14.54: The muon momentum distribution for neutrino $\mu+N p$ data events after calorimetric reconstruction (red) and the background events coming from the MC (black). The plots are scaled appropriately to indicate the relative percentage of background. 


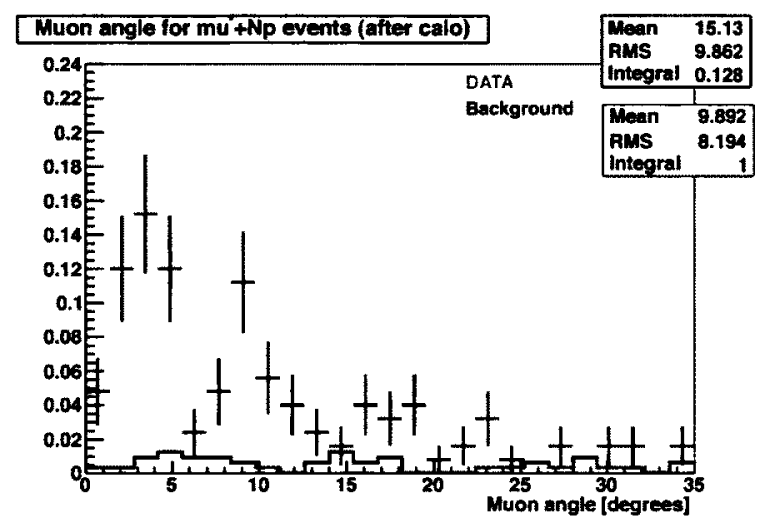

Figure 14.55: The muon angular distribution for neutrino $\mu+N p$ data events after calorimetric reconstruction (red) and the background events coming from the MC (black). The plots are scaled appropriately to indicate the relative percentage of background.

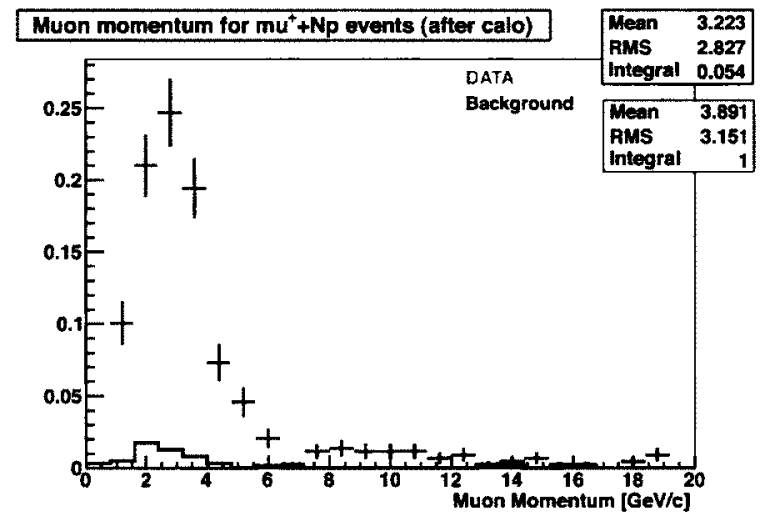

Figure 14.56: The muon momentum distribution for anti-neutrino $\mu+N p$ data events after calorimetric reconstruction (red) and the background events coming from the MC (black). The plots are scaled appropriately to indicate the relative percentage of background.

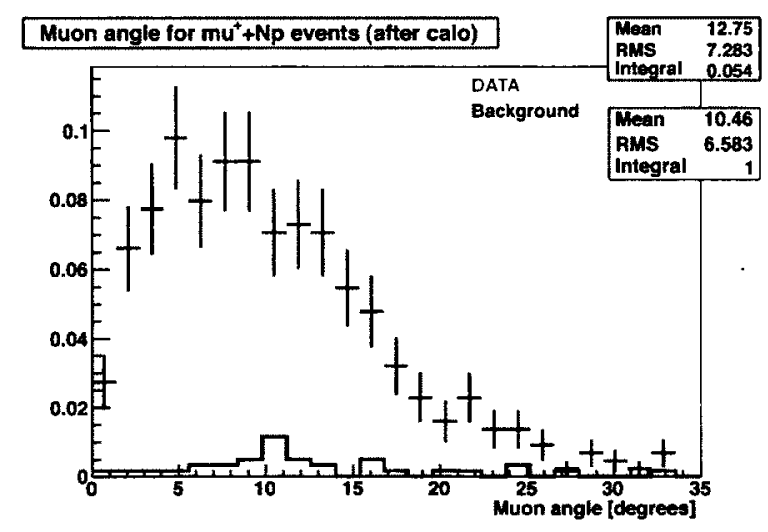

Figure 14.57: The muon angular distribution for anti-neutrino $\mu+N p$ data events after calorimetric reconstruction (red) and the background events coming from the MC (black). The plots are scaled appropriately to indicate the relative percentage of background. 


\subsubsection{Proton Kinematics}

ArgoNeuT with its low energy threshold of $21 \mathrm{MeV}$ of kinetic energy can reconstruct proton kinematics, which really shows the power of LArTPC technology. In other neutrino detectors, this reconstruction is not possible and these low energy protons are usually ignored. Proton length, kinetic energy and angle is reconstructed and compared with GENIE prediction for $1 p$ and $2 p$ events, higher multiplicities are not shown due to low statistics. The reconstructed quantities together with the $\mathrm{MC}$ prediction for $\mathrm{N}=1$ multiplicity are shown in Figure 14.58 for neutrinos. The protons in the MC are modeled as much more energetic and thus having longer tracks that what is observed in the data. Proton angle does not agree in terms of shape very well but the mean value is close to the MC prediction. A similar behaviour is seen for $\mathrm{N}=1$ anti-neutrino events as shown in Figure 14.59 where much less energetic protons are present in the data. Here, however, the angular distribution differs in both the mean and the shape with respect to the $\mathrm{MC}$. For $\mu+2 \mathrm{p}$ neutrino events protons are also less energetic than GENIE predicts and with the lower angle with respect to the beam as shown in Figure 14.60. Anti-neutrino $\mu+2$ p events are the ones that show an opposite signature and are more energetic, with longer tracks as modeled by GENIE.

From the angular distributions of protons the fraction of backwards and forward going protons can be extracted which further can tell us about the intra-nuclear interactions. These fractions are shown for $\mathrm{N}=1 \& 2$ multiplicity events for neutrinos and anti-neutrino $\mu+N p$ events as shown in Table 14.18. GENIE models well the fraction of backward going protons for $\mu+1 \mathrm{p}$ neutrino and anti-neutrino events. However, it predicts too many backward going protons for $\mu+2 p$ events. 

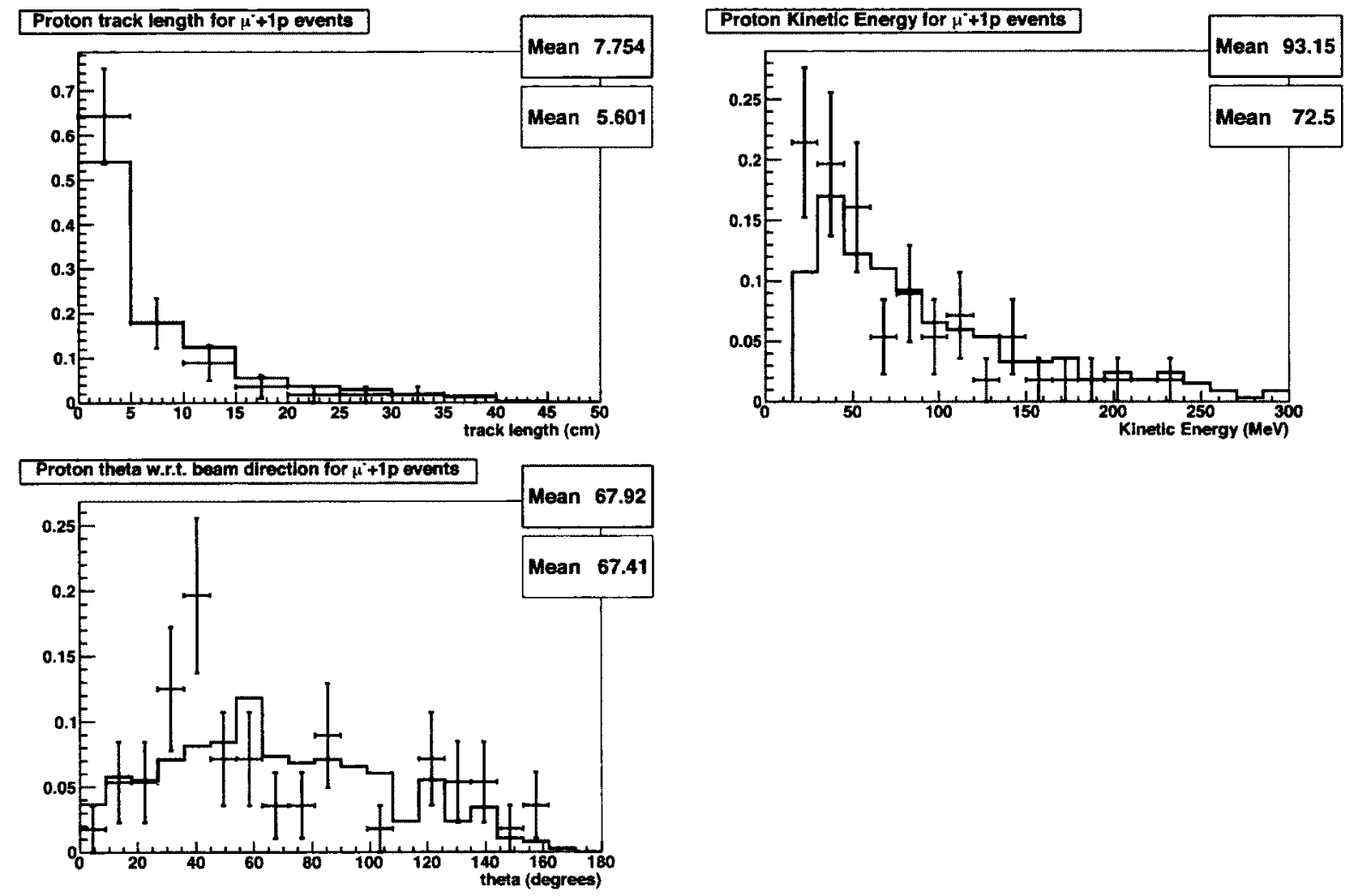

Figure 14.58: Distribution of proton length, kinetic energy and angle with respect to the beam direction for $\mu+1 \mathrm{p}$ neutrino events. In data this corresponds to sample of $\mathrm{N}=1$ events after calorimetry and in the MC to true $\mu+1 \mathrm{p}$ neutrino events after all cuts. 

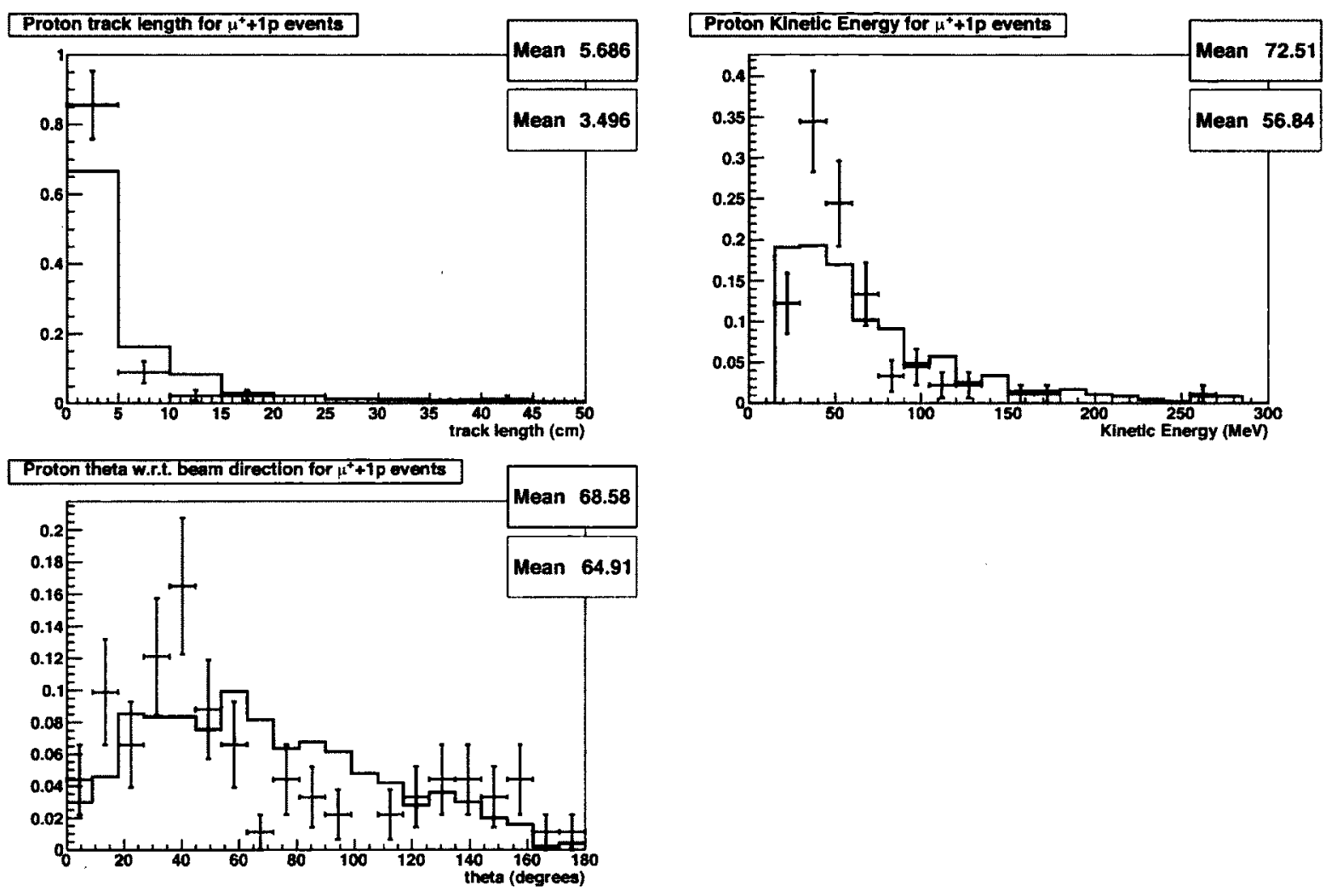

Figure 14.59: Distribution of proton length, kinetic energy and angle with respect to the beam direction for $\mu+1 \mathrm{p}$ anti-neutrino events. In data this corresponds to sample of $\mathrm{N}=1$ events after calorimetry and in the $\mathrm{MC}$ to true $\mu+1 \mathrm{p}$ neutrino events after all cuts. 

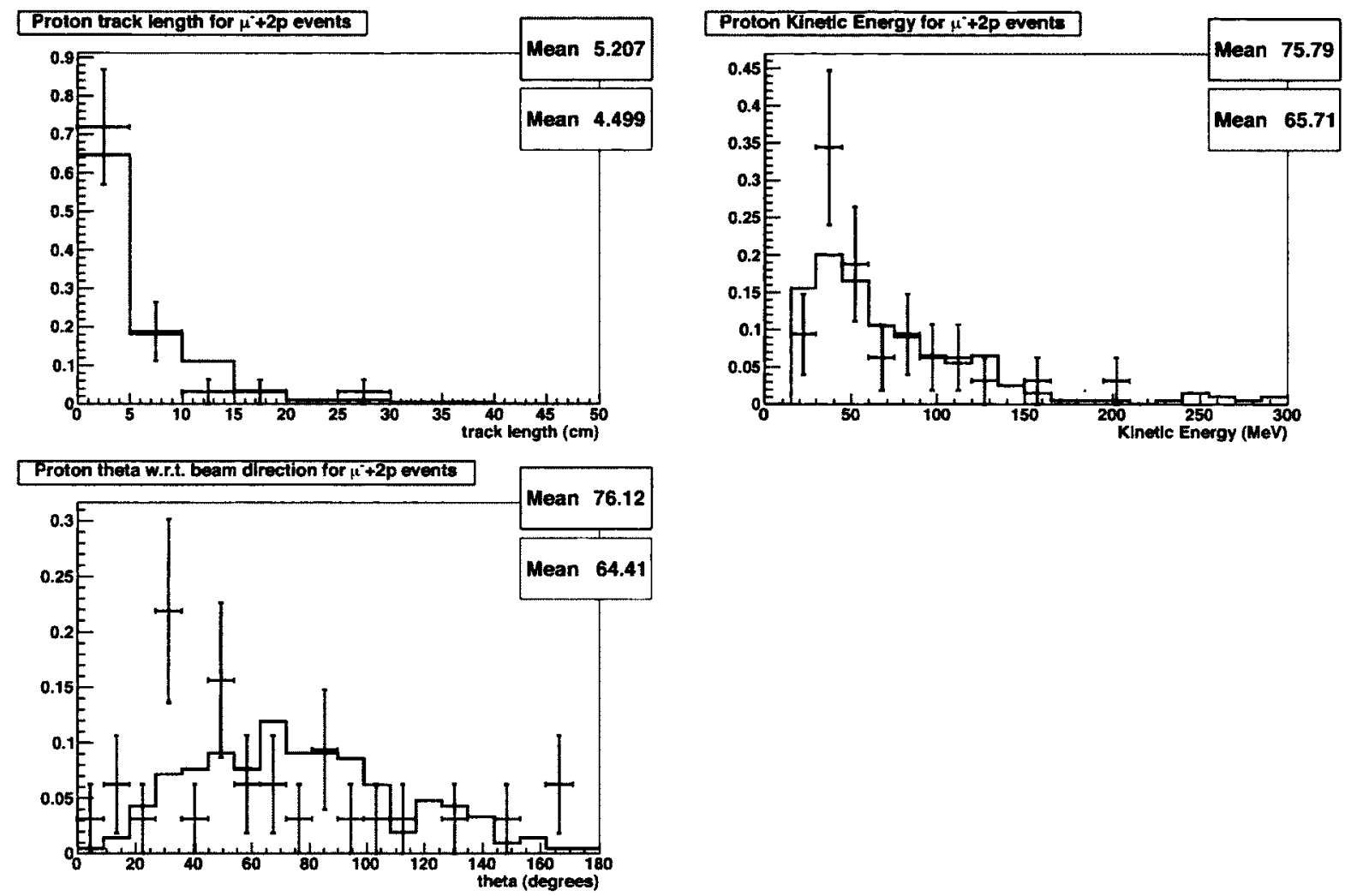

Figure 14.60: Distribution of proton length, kinetic energy and angle with respect to the beam direction for $\mu+2 \mathrm{p}$ neutrino events. In data this corresponds to sample of $\mathrm{N}=2$ events after calorimetry and in the $\mathrm{MC}$ to true $\mu+2 \mathrm{p}$ neutrino events after all cuts. 

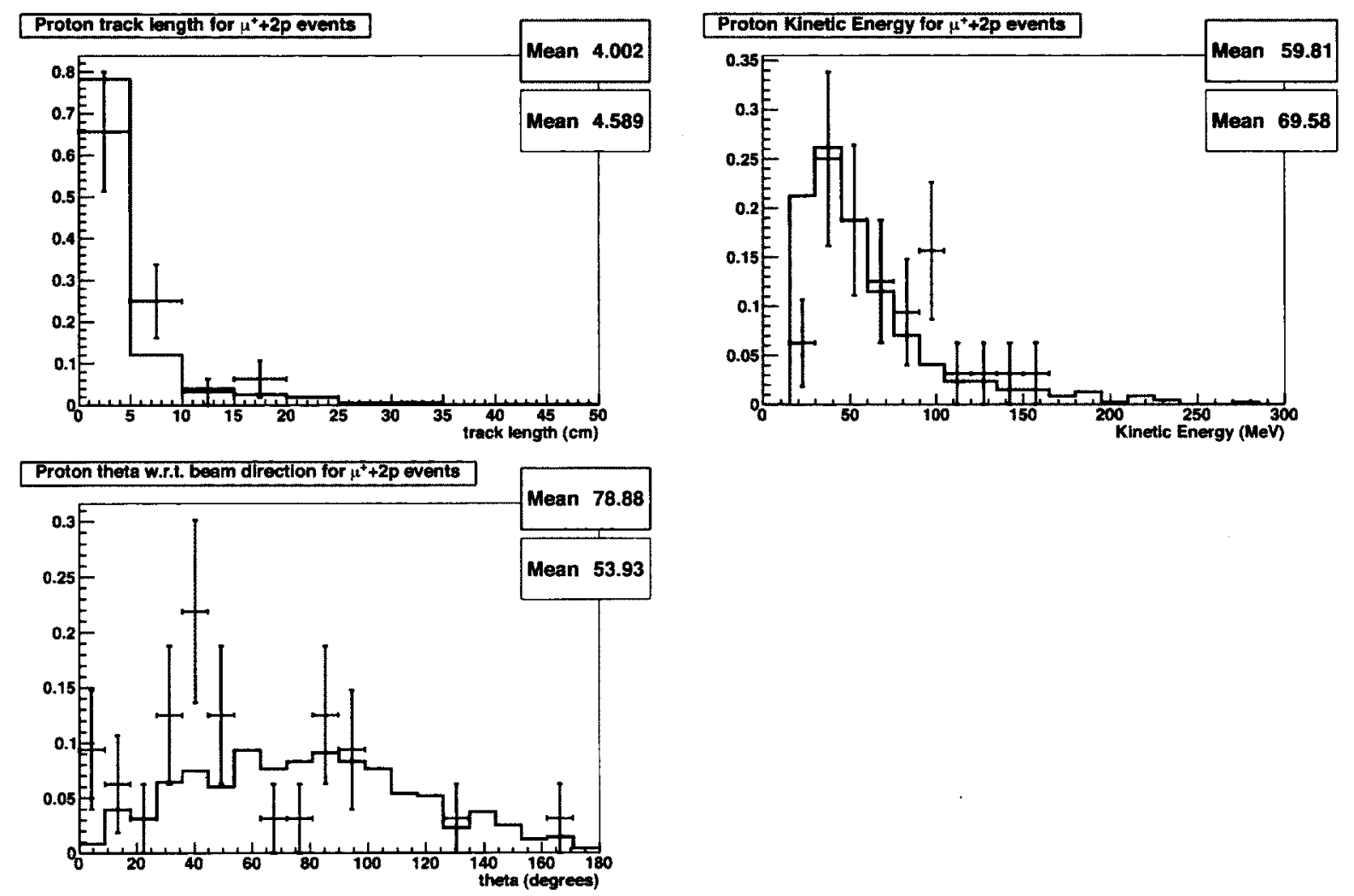

Figure 14.61: Distribution of proton length, kinetic energy and angle with respect to the beam direction for $\mu+2 \mathrm{p}$ anti-neutrino events. In data this corresponds to sample of $\mathrm{N}=2$ events after calorimetry and in the $\mathrm{MC}$ to true $\mu+2 \mathrm{p}$ neutrino events after all cuts. 


\begin{tabular}{|c|l|l|}
\hline Interaction & $\begin{array}{l}\text { frac. of backward going } \\
\text { protons in DATA }\end{array}$ & $\begin{array}{l}\text { frac. of backward going } \\
\text { protons in MC }\end{array}$ \\
\hline $1 \mathrm{p}+\mu^{-}$ & 0.25 & 0.26 \\
\hline $1 \mathrm{p}+\mu^{+}$ & 0.26 & 0.27 \\
\hline $2 \mathrm{p}+\mu^{-}$ & 0.22 & 0.30 \\
\hline $2 \mathrm{p}+\mu^{+}$ & 0.16 & 0.36 \\
\hline
\end{tabular}

Table 14.18: Fractions of backward going protons in the DATA and GENIE for N=1,2 multiplicity for neutrinos and anti-neutrinos.

\subsection{Concluding Remarks}

The dissertation presented here describes the topological analysis of charged current $\mu+N p$ neutrino events with the ArgoNeuT detector. This analysis has utilized about five months of the ArgoNeuT anti-neutrino mode data taking, collecting approximately $1.25 \times 10^{20}$ POT. The measurement of proton multiplicity at the vertex is the first such measurement in neutrino physics and made possible due to the use of the superb LArTPC technology. The reconstruction of proton kinematics with a low threshold of $21 \mathrm{MeV}$ of kinetic energy is also a new addition to the understanding of the neutrino interactions.

The $\mu+N p$ analysis is the first ArgoNeuT measurement that makes use of the calorimetric reconstruction, implemented by the ArgoNeuT collaboration, which is a significant step forward in the further demonstration of the power of the LArTPCs and the study of the vertex activity. Furthermore, it is a crucial step for the whole neutrino community as we have new results demonstrating the importance of nuclear effects in neutrino interactions that can be used to compare with and tune various MC models. It was shown that the used Monte Carlo generator, GENIE, is not with the exact agreement with the data, which is especially apparent for the higher proton multiplicities. It was also seen that overall the MC predicts more energetic protons with respect to what is found in the data. Thus, ArgoNeuT can be seen as the first step in an effort to tune various MC generators and understand where the differences come from.

The analysis of $\mu+N p$ events utilizes the use of the visual scanning technique as LArTPC event display features beautiful bubble-like-chamber images of neutrino interactions. By first studying these interactions visually, one learns about the specific features that need to be implemented in the future algorithms. Once implemented, one could make fully automated reconstruction of the more challenging neutrino event topologies. In the near future 
such algorithms could be employed for the analysis of $\mu+N p$ events with the MicroBooNE detector.

The size of MicroBooNE detector and the planned time of running would significantly improve the statistics of the $\mu+N p$ analysis . There is also much room for improvement in terms of the reconstruction algorithms. Once the reconstruction chain becomes fully automated, the cuts can be relaxed and focused more on the higher multiplicity proton events. Furthermore, a bigger detector, such as MicroBooNE, could also take into account the neutrons that have reinteracted in the liquid argon, as ArgoNeuT is too small to contain them.

The idea of the topological analysis presented here proves that it is far less MCdependent measurement than an analysis of defined by MC processes, for example Charged Current Quasi Elastic (CCQE) interactions. By avoiding large MC corrections, such as pion background, it provides a new approach to performing a neutrino analysis. This approach can be used for the neutrino oscillation experiments to improve the systematic error due to, for example, the flux differences between the near and far detectors. Furthermore, this analysis can be utilized by other experiments that have shared the NuMI beam line with ArgoNeuT, such as Miner $\nu$ a and MINOS. 


\section{Bibliography}

[1] W. Pauli letter of the 4th of December 1930.

[2] E. Fermi, Nuovo Cim. 11 (1934), 1.

[3] E. Fermi, Z. Physik 88 (1934), 161.

[4] C.L. Cowan Jr., F. Reines, F.B. Harrison, H.W. Kruse and A.D. McGuire, Science 124 (1956), 103.

[5] G. Danby et al., Phys. Rev. Lett. 9 (1962), 36.

[6] K. Kodama et al., Phys. Lett. B 504 (2001), 218.

[7] [ADELPH et al. Collaborations], Precision Electroweak Measurements on the Z Resonance, Phys. Rept. 427 (2006), 257 .

[8] C. S. Wu et al., Phys. Rev. 105 (1957), 1413.

[9] B. Kayser, Neutrino Physics, arXiv:hep-ph/0506165v1 (2005).

[10] R. Davis, Prog. Part. Nucl. Phys. 32 (1994), 13.

[11] B. Pontecorvo, Chalk River Lab. report PD-205, (1946).

[12] A.I. Abazov et al., [SAGE Collaboration], Phys. Rev. Lett. 67 (1991), 3332.

[13] J. N. Abdurashitov et al. [SAGE Collaboration], J. Exp. Theor. Phys. 95 (2002), 181.

[14] W. Hampel et al. [GALLEX Collaboration], Phys. Lett. B 447 (1999), 127.

[15] P. Anselmann et al., [GALLEX Collaboration], Phys. Lett. B 285 (1992), 376. 
[16] M. Altmann et al., [GNO Collaboration.], Phys. Lett. B 616 (2005), 174.

[17] K.S. Hirata et al., [Kamiokande Collab.], Phys. Rev. Lett. 63 (1989), 16.

[18] Y. Fukuda et al., [Super-Kamiokande Collab.], Phys. Rev. Lett. 81 (1998), 1158.

[19] J. Hosaka et al., [Super-Kamiokande Collab.], Phys. Rev. D 73 (2006), 112001.

[20] J.P. Cravens et al., [Super-Kamiokande Collab.], Phys. Rev. D 78 (2008), 032002.

[21] K. Abe et al., [Super-Kamiokande Collab.], Phys. Rev. D 83 (2011), 052010.

[22] http://www.sno.phy.queensu.ca.

[23] B. Aharmim et al. [SNO Collaboration], arXiv:nucl-ex/0610020.

[24] J. N. Bahcall, M. H. Pinsonneault and S. Basu, Astrophys. J. 555 (2001), 990.

[25] T. Araki et al. [KamLAND Collaboration], Phys. Rev. Lett. 94 (2005), 081801.

[26] Y. Fukuda et al. [Super-Kamiokande Collaboration], Phys. Lett. B 433 (1998), 9.

[27] M.H. Ahn et al., [K2K Collaboration], Phys. Rev. D 74 (2006), 072003.

[28] D.G. Michael et al., [MINOS Collab.], Phys. Rev. Lett. 97 (2006), 191801.

[29] P. Adamson et al., [MINOS Collab.], Phys. Rev. Lett. 101 (2008), 131802.

[30] P. Adamson et al., [MINOS Collab.], Phys. Rev. Lett. 107 (2011), 021801.

[31] P. Adamson et al., [MINOS Collab.], Phys. Rev. Lett. 108 (2012), 191801.

[32] http://www.cern.ch/opera.

[33] N. Agafonova et al. [OPERA Collaboration], Phys. Lett. B 691(2010), 138.

[34] A. Aguilar et al. [LSND Collaboration], Phys. Rev. D 64 (2001), 112007.

[35] G.S. Abrams et al., Phys. Rev. Lett. 63 (1989), 2173.

[36] D. Decamp et al. [ALEPH Collaboration], Phys. Lett. B 231(1989), 519.

[37] A.A. Aguilar-Arevalo et al. [MiniBooNE Collaboration], Phys. Rev. Lett. 98 (2007), 231801. 
[38] A.A. Aguilar-Arevalo et al. [MiniBooNE Collaboration], Phys. Rev. Lett. 105 (2010), ’ 181801.

[39] D. Griffiths, Introduction to Elementary Particles, John Wiley and Sons, Inc., (1987).

[40] D. Casper, Nucl. Phys. Proc. Suppl. 112 (2002), 161.

[41] K. Nakamura, et al., J. Phys. G37 (2010), 075021.

[42] V. Bernard, et al., 2002, J. Phys. G. 28, R1.

[43] S. Frullani, and J. Mougey, Adv. Nucl. Phys. 14 (1984), 1.

[44] R. A. Smith, and E. J. Moniz, Nucl. Phys. B 43 (1972), 605.

[45] A. M. Ankowski and J. T. Sobczyk, Phys. Rev. C 74 (2006), 054316.

[46] O. Benhar, N. Farina, H. Nakamura, M. Sakuda, and R. Seki, Phys. Rev. D 72 (2005), 053005 .

[47] O. Benhar and D. Meloni, Nucl. Phys. A 789 (2007), 379.

[48] C. Juszczak, J. T. Sobczyk, and J. Zmuda, Phys. Rev. C 82 (2010), 045502.

[49] H. Nakamura and R. Seki, , Nucl. Phys. Proc. Suppl. 112 (2002), 197.

[50] J. E. Amaro, M. B. Barbaro, J. A. Caballero, T. W. Donnelly, A. Molinari, and I. Sick,, Phys. Rev. C 71 (2005), 015501.

[51] T. Leitner, O. Buss, L. Alvarez-Ruso, and U. Mosel, Phys.Rev. C 79 ( 2009), 034601.

[52] J. Nieves, J. E. Amaro, and M. Valverde, Phys.Rev. C 70 (2004), 055503.

[53] A. Butkevich, , Phys.Rev. C 82 (2010), 055501.

[54] A. Aguilar-Arevalo, et al., Phys. Rev. D 81(2010), 092005.

[55] A. Aguilar-Arevalo, Phys. Rev. Lett. 100 (2008), 032301.

[56] M. Dorman, AIP Conf. Proc. 1189 (2009), 133.

[57] X. Espinal and F. Sanchez, AIP Conf. Proc. 967 (2007), 117.

[58] R. Gran, et al., Phys. Rev. D 74 (2006), 052002. 
[59] V. Lyubushkin, et al., Eur. Phys. J. C 63 (2009), 355.

[60] J. A. Formaggio, G. P. Zeller arXiv:1305.7513v1

[61] M. Martini, (2011), arXiv:1110.5895 [hep-ph].

[62] S. Bo, C. Giusti, F. Pacati, and M. Radici, Electro-magnetic Response of Nuclei (Clarendon Press, Oxford, 1996).

[63] M. Martini, M. Ericson, G. Chanfray, and J. Marteau, Phys. Rev. C 80 (2009), 06550, arXiv:0910.2622 [nucl-th].

[64] C. Rubbia, The liquid-argon time projection chamber: a new concept for neutrino detector, CERN-EP/77-08 (1977).

[65] ICARUS Collaboration, Design, construction and tests of the ICARUS T600 detector, Nucl. Instrum. Meth. in Phys. Res. A 527 (2004), 329.

[66] ICARUS Collaboration, Underground operation of the ICARUS T600 LAr-TPC: first results, Journal of Instrumentation, vol. 6 P07011 (2011).

[67] LBNE Collaboration, T. Akiri et. al., The 2010 Interim Report of the Long-Baseline Neutrino Experiment Collaboration Physics Working Groups, arXiv: 1110.6249 [hepex].

[68] O. Bunemann and J.A. Harvey, Can. J. Res. 27 (1949), 191.

[69] C.D. Curtis, G.M. Lee, C.W. Owen, C.W. Schmidt and W.M. Smart, Linac H- beam operation and uses at Fermilab, IEEE Trans. Nucl. Sci. 26 (1979), 3760.

[70] C.W. Schmidt, The Fermilab $400 \mathrm{MeV}$ Linac upgrade, in Proceedings of 1993 Particle Accelerator Conf., Washington U.S.A. (1993), 1655.

[71] Detailed description of the Booster Beam in Rookie Books, http://www- bdnew.fnal.gov/operations/rookie books/Booster V4.1.pdf.

[72] Detailed description of the Main Injector in Rookie Books, http://www- bdnew.fnal.gov/operations/rookie books/Main Injector v1.1.pdf.

[73] P. Adamson et al. [MINOS Collaboration], An improved measurement of muon antineutrino disappearance in MINOS, Phys. Rev. Lett. 108, (2012) 191801. 
[74] B. Rebel et al., Results from the Fermilab Materials Test Stand and Status of the Liquid Argon Purity Demonstrator, FERMILAB-CONF-10-692-PPD.

[75] D. Edmunds, http://www.pa.msu.edu/?edmunds/LArTPC.

[76] P. Adamson et al., Nucl. Inst. \& Meth. A 492, (2002) 325 and A 521, (2004) 36.

[77] D.G. Michael et al. [MINOS Collaboration], Phys. Rev. Lett. 97, (2006) 191801.

[78] G. Bakale et al., Effects of an Electric Field on Electron Attachment to $S F_{6}, N_{2} O$ and $\mathrm{O}_{2}$ in Liquid Argon and Xenon, J. Phys. Chem. 80 (1976), 2556.

[79] ICARUS Collaboration, Argon purification in the liquid phase, Nucl. Instrum. and Meth. in Phys. Res. A 333 (1993), 567.

[80] A. Curioni et al., A Regenerable Filter for Liquid Argon Purification, Nucl. Instrum. and Meth. in Phys. Res. A 605 (2009), 306.

[81] G. Carugno et al., Nucl. Instr. Meth. A 292 (1990), 580.

[82] PolyMicro Technologies Inc., FVP600660710UVMI.

[83] G. Bakale et al., Journal of Physical Chemistry 8023 (1976).

[84] C. Anderson et al., Analysis of a large sample of neutrino-induced muons with the ArgoNeuT detector, 2012 JINST 7 P10020.

[85] ArgoNeuT Collaboration, First Measurements of Inclusive Muon Neutrino Charged Current Differential Cross Sections on Argon, Phys. Rev. Lett. 108 (2012),161802.

[86] A.A. Aguilar-Arevalo et al., Phys. Rev. Lett. 98 (2007), 231801.

[87] A.A. Aguilar-Arevalo et al. , Phys. Rev. Lett. 102 (2009), 101802.

[88] http://www.sanfordlab.org/

[89] C. Andreopoulos et al., Nucl. Instrum. Meth. A 614 (2010), 87.

[90] A. Bodek and J. L. Ritchie, Further studies of fermi motion effects in lepton scattering from nuclear targets, Phys. Rev., D 24, (1981) 1400. 
[91] E. J. Moniz et al., Nuclearfermimomentafromquasielasticelectronscattering, Phys. Rev. Lett., 26, (1971) 445448.

[92] J. Finn et al., Nucl. Phys. A 274 (1976), 28.

[93] D. S. Baranov et al., An estimate for the formation length of hadrons in neutrino interactions, PHE 84-04, 1984.

[94] G. D. Harp, Extension of the isobar model for intranuclear cascades to $1 \mathrm{GeV}$, Phys. Rev., C 10 (1974), 23872396.

[95] S. G. Mashnik, A. J. Sierk, K. K. Gudima, M. I. Baznat, and N. V. Mokhov LANL Report LA-UR-05-7321 (2005), RSICC Code Package PSR-532.

[96] S. G. Mashnik, A. J. Sierk, K. K. Gudima, and M. I. Baznat, CEM03 and LAQGSM03: New modeling tools for nuclear applications, J. Phys. Conf. Ser.,41 (2006), 340351.

[97] D. Ashery et al., True absorption and scattering of pions on nuclei, Phys. Rev., C 23 (1981), 21732185.

[98] I. Navon et al., True absorption and scattering of $50 \mathrm{MeV}$ pions, Phys. Rev., C 28 (1983), 2548.

[99] A. S. Carroll et al., Pion-Nucleus Total Cross-Sections in the (3,3) Resonance Region, Phys. Rev. C 14 (1976), 635638.

[100] A. S. Clough et al., Pion-Nucleus Total Cross-Sections from 88-MeV to 860 MeV,Nucl. Phys. B 76 (1974), 15.

[101] W. Bauhoff At. Data and Nucl. Data Tables, 35 (1986), 429.

[102] C. H. Llewellyn Smith, Neutrino reactions at accelerator energies, Phys. Rept., 3 (1972), 261.

[103] H. Budd, A. Bodek, and J. Arrington, Modeling quasi-elastic form factors for electron and neutrino scattering, hep-ex/0308005, 2003.

[104] L. A. Ahrens et al., Measurement of Neutrino-Proton and anti-neutrino-Proton Elastic Scattering, Phys. Rev., D 35 (1987), 785. 
[105] D. Rein and L. M. Sehgal, Coherent $\pi^{0}$ production in neutrino reactions, Nucl. Phys., B 223 (1983), 29.

[106] W. M. Yao et al., Review of particle physics, J. Phys., G 33 (2006),11232.

[107] D. Rein and L. M. Sehgal, PCAC and the deficit of forward muons in $\pi^{+}$production by neutrinos, Phys. Lett., B 657 (2007), 207209.

[108] A. Bodek and U. K. Yang, Higher twist, $\xi_{w}$ scaling, and effective LO PDFs for lepton scattering in the few GeV region, J. Phys., G 29(2003),18991906.

[109] L. W. Whitlow, S. Rock, A. Bodek, E. M. Riordan, and S. Dasu, A Precise extraction of $R=\sigma_{L} \sigma_{T}$ from a global analysis of the SLAC deep inelastic e $p$ and e $d$ scattering cross-sections, Phys. Lett., B 250 (1990),193198.

[110] D. Rein and L. M. Sehgal, Neutrino excitation of baryon resonances and single pion production, Ann. Phys., 133 (1981), 79.

[111] R. P. Feynman, M. Kislinger, and F. Ravndal, Current matrix elements from a relativistic quark model, Phys. Rev.,D 3 (1971), 27062732.

[112] ROOT, Nucl. Inst. and Meth. in Phys. Res. A 389 (1997) 81 and also http://root.cern.ch/

FFT, Fast Fourier Transform and Convolution Algorithms, New York: Springer- Ver$\operatorname{lag}(1982)$.

Optimal Filter, Theory and Application of Digital Signal Processing, Englewood Cliffs, NJ: Prentice-Hall (1975).

DBSCAN, Proc. 2nd Int. Conf. on Knowledge Discovery and Data Mining (KDD-96), AAAI Press (1996), 226.

HOUGH Transform, Proc. Int. Conf. on High Energy Accelerators and Instrumentation (1959).

[113] J. Birks, Theory and Practice of Scintillation Counting, Pergamon Press, 1964.

[114] MINOS Collaboration, The magnetized steel and scintillator calorimeters of the MINOS experiment, Nucl. Instrum. and Meth. in Phys. Res. A 596 (2008), 190. 
MINOS Collaboration, Study of muon neutrino disappearance using the Fermilab Main Injector neutrino beam, Phys. Rev. D 77, (2008) 072002.

MINOS Collaboration, Neutrino and antineutrino inclusive charged-current cross section measurements with the MINOS near detector, Phys. Rev. D 81 (2010) 072002.

[115] W. Walkowiak, Drift velocity of free electrons in liquid argon, Nucl. Instrum. and Meth. in Phys. Res. A 449 (2000), 288.

[116] H. Gallagher, G. Garvey, and G.P. Zeller Neutrino-Nucleus Interactions, Annual Review 61 (2011), 355.

[117] J. Spitz, Measuring Muon-Neutrino Charged-Current Differential Cross Sections with a Liquid Argon Time Projection Chamber, Ph.D. Thesis, Yale University (2011).

[118] A. McGowan, Observation of a Deficit in NuMI Neutrino-induced Rock and Nonfudical Muons in the MINOS Far Detector and Measurement of Neutrino Oscillation Parameters, Ph.D. Thesis. University of Minnesota (2003).

[119] A. Ferrari, P. R. Sala, A. Fasso, and J. Ranft, CERN-2005-010.

[120] MINOS Collaboration, Phys. Rev. D 77, (2008) 072002.

[121] MINER $\nu$ A Collaboration, Phys. Rev. Lett. 111, (2013) 022501.

[122] Z. Pavlovic, Observation of Disappearance of Muon Neutrinos in the NuMI Beam, Ph.D. Thesis, University of Texas (2008). 